\title{
SHAPING FUNCTIONAL GUT MICROBIOTA USING DIETARY BIOACTIVES TO REDUCE COLON CANCER RISK
}

\author{
A Dissertation \\ by \\ DEREK VAUGHN SEIDEL \\ Submitted to the Office of Graduate and Professional Studies of \\ Texas A\&M University \\ in partial fulfillment of the requirements for the degree of \\ DOCTOR OF PHILOSOPHY
}

$\begin{array}{ll}\text { Chair of Committee, } & \text { Nancy D. Turner } \\ \text { Committee Members, } & \text { Robert S. Chapkin } \\ & \text { Tri Duong } \\ & \text { Arul Jayaraman } \\ \text { Intercollegiate Faculty Chair, } & \text { David W. Threadgill }\end{array}$

May 2019

Major Subject: Genetics

Copyright 2018 Derek V. Seidel 


\begin{abstract}
Alterations in gut microbiota and metabolome occur during colon carcinogenesis and metabolic syndrome, but these changes may be reduced by exposure to bioactive compounds in foods. We hypothesized that bioactive compound-rich diets improve colon microbiota and metabolism to mitigate: 1) azoxymethane- (AOM) induced colon carcinogenesis in rats, 2) HZE radiation-induced alterations in the microbiota in mice, and 3) risk factors that define metabolic syndrome in human subjects.
\end{abstract}

A dried plum (DP) containing diet reduced preneoplastic lesions and led to lower Nos2, Tlr2 and Tlr4 expression than basal diet counterparts. DP increased Bacteroidetes and decreased Firmicutes in the distal colon, and increased Actinobacteria and decreased Lactobacillus throughout the colon. DP reduced fecal short chain fatty acid (SCFA) concentrations and increased luminal concentrations of compounds derived from microbial metabolism of plant derivatives. Microbial metagenome prediction revealed alterations in KEGG pathways related to metabolism and pathway analysis of distal metabolites suggests DP altered critical regulators of colon carcinogenesis and inflammation.

Exposure to 1 Gy of Fe (RAD) did not affect luminal SCFA, however, DP+RAD mice had a lower percentage of propionate compared to DP+SHAM, control diet (CD)+SHAM and $\mathrm{CD}+\mathrm{RAD}$ groups. RAD increased the abundance of Akkermansia; however, DP increased Akkermansia, Lachnospiraceae, Anaeroplasma, and Coriobacteriaceae and reduced Oscillospira, Ruminococcaceae, Lactobacillus, and Clostridiaceae. Evidence of an interaction between DP and radiation exposure in $40 \%$ of animals warrants further investigation. 
Sumac sorghum (SS) increased the anti-inflammatory genus Faecalibacterium, but had no effect on serum markers of inflammation, blood lipids, fasting blood sugar, percentage body fat, or fecal SCFA. Metabolomics of plasma showed an increase in 3-hydroxyhippurate following SS consumption, and a reduction of three gamma-glutamyl AAs. Targeted fecal metabolite quantification revealed increased concentrations of 3-(3-hydroxyphenol)propionic, 3phenylpropionic, and benzoic acid, and reduced quinic acid following $100 \mathrm{~g} /$ day SS consumption. Correlation between plasma metabolites and fecal microbes revealed hippurate was positively correlated with Akkermansia, while Faecalibacterium was negatively associated with several gamma-glutamyl amino acids.

Both DP and SS modified the colon metabolome and microbiome, increased putatively beneficial bacteria and microbially-derived compounds associated with positive health outcomes. Causal investigations and interactions between the DP and radiation are warranted. 


\section{DEDICATION}

This dissertation is dedicated to my family and friends. 


\section{CONTRIBUTORS AND FUNDING SOURCES}

This work was supported by a dissertation committee consisting of Professor(s) Nancy D. Turner (Chair), and Robert S. Chapkin of the Department of Nutrition and Food Science, Professor Tri Duong of the Poultry Science Department, and Professor Arul Jayaraman of the Department of Chemical Engineering.

The microbe sequencing in CHAPTER II and III was performed by Dr. M A. AzcaratePeril at the University of North Carolina, School of Medicine at Chapel Hill. The microbe sequencing work in CHAPTER IV was performed by Drs. Jens Walter and Ines Martinez at University of Nebraska-Lincoln. The metabolomics profiling presented in CHAPTER II and IV was performed by Metabolon Inc. (Durham, NC). Fatty acid analysis of diets presented in CHAPTER II was performed by Dr. Yang-Yi Fan. The primary experiment in CHAPTER III, from which we obtained samples, was conducted by Dr. Ann-Sofie Schreurs under Dr. Ruth Globus at NASA AMES Research Center in California. The statistical analysis of the microbe and plasma metabolome data presented in CHAPTER IV was carried out by Dr. Maria Joseph with direction from Dr. Raymond Carroll. Dr. Mark Haub at Kansas State University conducted the experiment presented in CHAPTER IV as well as the assessment of glucose and insulin levels in the subjects' blood samples. The antioxidant activity of the sumac sorghum in

CHAPTER IV was assessed by Dr. L. Rooney at Texas A\&M University and the lipid bioactive components were assessed by Dr. T. Carr at the University of Nebraska-Lincoln. Dr. Carr also conducted the lipid profiling of subjects' plasma samples. Dr. Cory Klemashevich and Smriti Shankar developed the method and performed the fecal metabolite quantification following 
sample extraction in CHAPTER IV. Beth Juliar carried out the statistical analyses presented in CHAPTER IV/V with direction from Dr. Raymond Carroll.

All other work conducted for the dissertation was completed by the student

independently. Sections of CHAPTER I are reproduced from a published review article (1). As a journal author, I retain the rights for large number of author uses, including use by the employing institute (Texas A\&M University). These rights are retained and permitted without the need to obtain specific permission from Elsevier.

Derek Seidel was supported by the NSBRI Mentored Research Program (EO02001)

funded by NASA (NCC 9-58). The work presented in Chapters II and III was supported by the California Dried Plum Board (PN 12-20). The work presented in Chapters IV and V was supported by the United Sorghum Checkoff Program (R0002-11). 


\section{NOMENCLATURE}

\begin{tabular}{|c|c|}
\hline $\mathrm{AC}$ & aberrant crypt \\
\hline $\mathrm{ACF}$ & aberrant crypt foci \\
\hline$\alpha \mathrm{PD}-\mathrm{L} 1$ & alpha programmed death-ligand 1 \\
\hline AhR & aryl hydrocarbon receptor \\
\hline $\mathrm{AOM}$ & azoxymethane \\
\hline APC & adenomatous polyposis coli \\
\hline Bcl-2 & b-cell lymphoma-2 \\
\hline BCL2L11 & Bcl-2-like protein 11 \\
\hline BRAF & B-Raf Proto-Oncogene, Serine/Threonine Kinase \\
\hline $\mathrm{CAC}$ & colitis-associated colon cancer \\
\hline CGAP & Cancer Genome Anatomy Project \\
\hline $\mathrm{CIN}$ & chromosomal instability \\
\hline CLA & conjugated linoleic acid \\
\hline CoA & coenzyme A \\
\hline COX-2 & cyclooxygenase- 2 \\
\hline CRC & colorectal cancer \\
\hline DHA & docosahexaenoic acid \\
\hline DIM & diindolylmethane \\
\hline $\mathrm{DMH}$ & dimethylhydrazine \\
\hline DNA & deoxyribonucleic acid \\
\hline DSB & double strand break \\
\hline
\end{tabular}




\begin{tabular}{|c|c|}
\hline DSS & dextran sulfate sodium \\
\hline EGF & epidermal growth factor \\
\hline EPA & eicosapentaenoic acid \\
\hline FICZ & formylindolo-[3,2-b]-carbazole \\
\hline GO & gene ontology \\
\hline HAT & histone acetyltransferase \\
\hline HDAC & histone deacetylase \\
\hline HMACF & high multiplicity aberrant crypt foci \\
\hline hMLH1 & human MutL homolog 1 \\
\hline IBD & inflammatory bowel disease \\
\hline IFN- $\gamma$ & interferon gamma \\
\hline $\mathrm{IgA} / \mathrm{G}$ & immunoglobulin $\mathrm{A} / \mathrm{G}$ \\
\hline iNOS & inducible nitric oxide synthase \\
\hline $\mathrm{I} 3 \mathrm{C}$ & indole-3-carbinol \\
\hline JNK & c-jun N-terminal kinase \\
\hline KEGG & Kyoto Encyclopedia of Genes and Genomes \\
\hline KRAS & V-Ki-Ras2 Kirsten Rat Sarcoma 2 Viral Oncogene Homolog \\
\hline LAB & lactic acid bacteria \\
\hline LCM & laser capture microdissection \\
\hline $\operatorname{Lgr}^{+}$ & leucine-rich-repeat-containing G-protein-coupled receptor 5 positive \\
\hline MAPK & mitogen-activated protein kinase \\
\hline MCT1 & monocarboxylate transporter 1 \\
\hline $\mathrm{mM}$ & millimolar \\
\hline
\end{tabular}




\begin{tabular}{ll} 
MOMP & mitochondrial outer membrane permeabilization \\
MSI & microsatellite instability \\
NLR & NOD-like receptor \\
NF-kB & nuclear transcription factor kappa B \\
OTU & operational taxonomic unit \\
qPCR & quantitative polymerase chain reaction \\
PUFA & polyunsaturated fatty acid \\
ROS & reactive oxygen species \\
rRNA & ribosomal ribonucleic acid \\
SCFA & short chain fatty acid \\
Slc16a1 & proton-coupled monocarboxylate transporter 1 (MCT1) \\
Slc5a8 & sodium-coupled monocarboxylate transporter 1 \\
STAT1 & signal transducer and activator of transcription 1-alpha/beta \\
TCDD & 2,3,7,8-tetrachloro-dibenzo-p-dioxin \\
TGF- $\beta$ & transforming growth factor beta \\
TLR & toll-like receptor \\
TNBS & trinitrobenzene sulfonic acid \\
TNF & tumor necrosis factor \\
TRAIL & TNF-related apoptosis inducing ligand \\
4-HNE & trans-4-hydroxy-2-nonenol \\
\hline
\end{tabular}




\section{TABLE OF CONTENTS}

Page

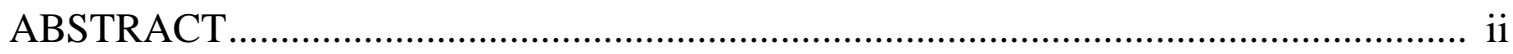

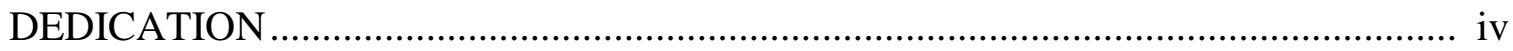

CONTRIBUTORS AND FUNDING SOURCES ……………….............................. v

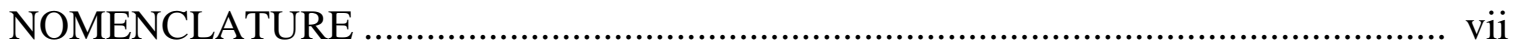

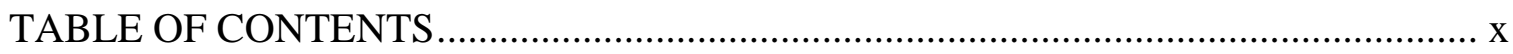

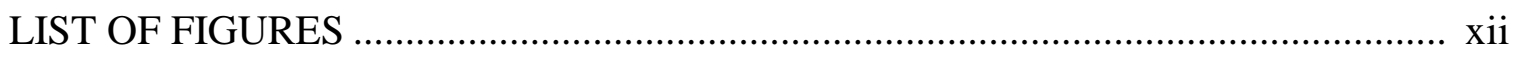

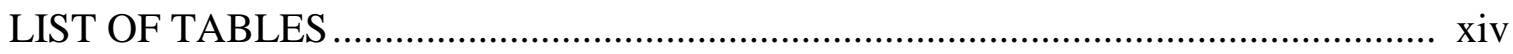

CHAPTER I INTRODUCTION AND LITERATURE REVIEW .................................... 1

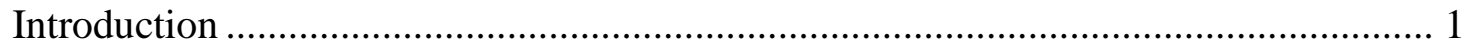

Literature Review ………………………………........................................... 3

Hypothesis and Specific Aims ………………………...................................... 28

CHAPTER II DRIED PLUMS PROTECT AGAINST CHEMICALLY-INDUCED

COLON CARCINOGENESIS IN SPRAGUE DAWLEY RATS ................................. 31

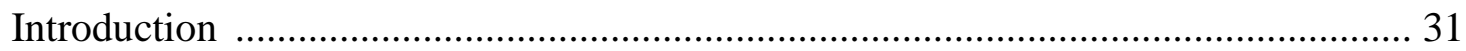

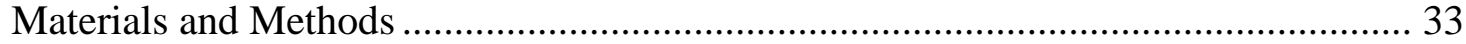

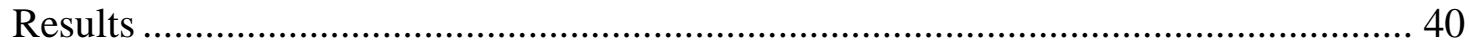

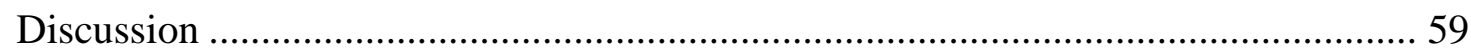

CHAPTER III DRIED PLUMS MODIFY COLON MICROBIOTA AND LUMINAL

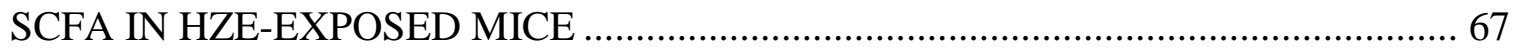

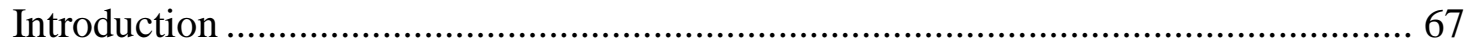

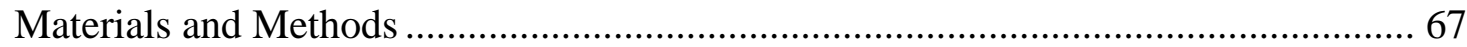

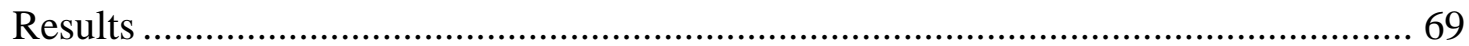

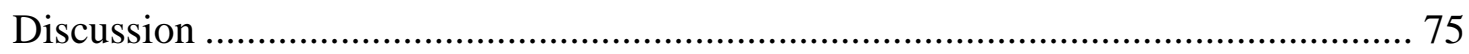

CHAPTER IV POLYPHENOL-RICH SUMAC SORGHUM DIETARY

INTERVENTION ALTERS COLON MICROBIOTA AND PLASMA

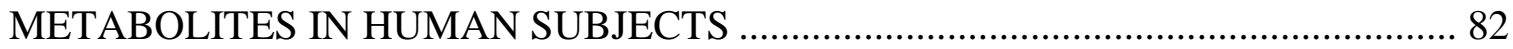

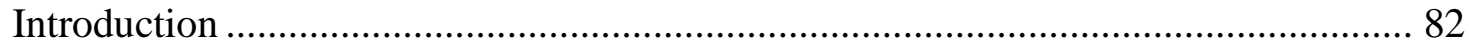

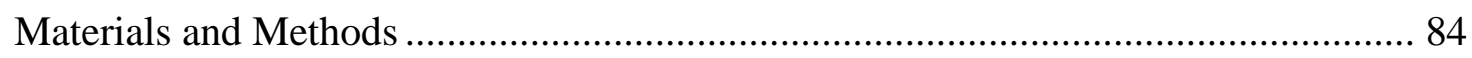


Results 92

Discussion

CHAPTER V RELATING THE MICROBIOME AND THE METABOLOME: A SECONDARY ANALYSIS OF THE KANSAS SORGHUM STUDY DATA ............. 112

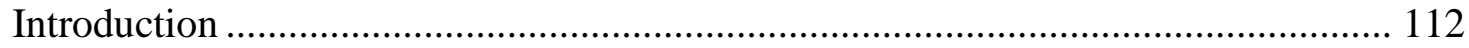

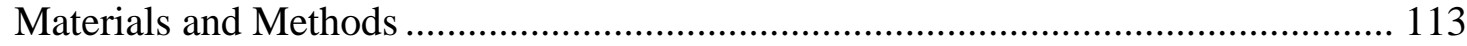

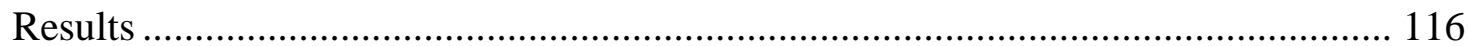

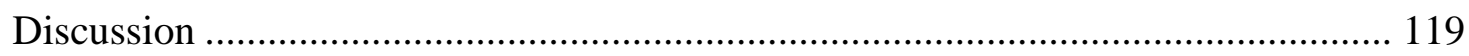

CHAPTER VI SUMMARY AND CONCLUSIONS …………………………......... 125

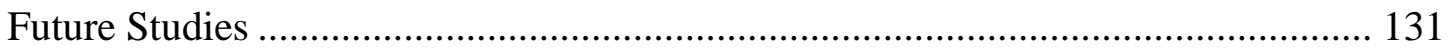

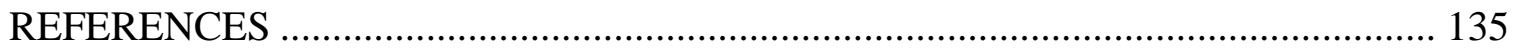

APPENDIX A TABLES AND FIGURES OF RESULTS ………………………........ 170

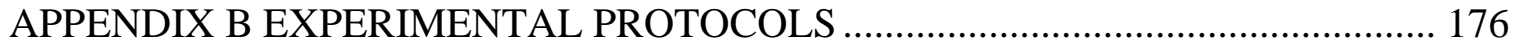




\section{LIST OF FIGURES}

Page

Figure 1. Timeline of AOM treatments for AOM group rats in each experimental group. Saline animals were injected with saline at the same time as their AOM counterparts. After 3 wk of experimental diets, AOM injections were given (15 mg/kg body weight, 1 wk separation, 2 injections total).

Figure 2. Daily food intake (averaged over $48 \mathrm{~h}$ ) at experimental day 20, 55, and 82.

At d 20, animals assigned to the plum diet had increased diet intake, compared to basal diet counterparts. Data are means \pm SEM. At each time point, bars not sharing a common letter differ significantly $(P<0.05)$.

Figure 3. Average body weights at experimental day 0, 21, 28, 56 and 84. At d 21 and 56, PD+S animals weighed more than BD+A animals. At d 84, $\mathrm{PD}+\mathrm{S}$ animals weighed more than $\mathrm{BD}+\mathrm{A}$ and $\mathrm{PD}+\mathrm{A}$ animals, and $\mathrm{BD}+\mathrm{S}$ animals weighed more than $\mathrm{BD}+\mathrm{A}$ animals. Data are means $\pm \mathrm{SEM}$. At each time point, bars not sharing a common letter differ significantly $(P<0.05)$.

Figure 4. Percent abundance of major bacterial orders by diet/injection group and location. Bars without a common letter are significantly different $(P<0.05)$. Data are means \pm SEM.

Figure 5. Unweighted UniFrac beta-diversity analysis illustrated with principle coordinate plots reveals separation of samples based on diet.

Figure 6. Weighted UniFrac beta-diversity analysis illustrated with principle coordinate plots reveals separation of samples based on diet with maximum separation occurring between distal colon microbial samples.

Figure 7. IPA workflow for network discovery using metabolites found to be significantly different between distal colon samples of Plum+AOM and Basal+AOM animals.

Figure 8. Graphical representation of the top ID Associated Network Functions generated by IPA: Free Radical Scavenging, Cell Death and Survival, Reproductive System Development and Function. Arrows indicate associations between nodes. Red and green indicate increases and decreases in metabolites, respectively.

Figure 9. Relative abundance of bacterial phyla at $30 \mathrm{~d}$ post-IR in C57BL/6 mice consuming a control or dried plum diet and exposed to $1 \mathrm{~Gy}$ of ${ }^{56} \mathrm{Fe}$ $\mathrm{HZE}$ radiation or sham irradiated. 
Figure 10. Alpha diversity indices of species diversity (PD whole tree and Shannon) and species richness (Chaol and Observed species) at 1 month post-IR in C57BL/6 mice fed a control or dried plum diet and exposed to $1 \mathrm{~Gy}$ Fe ions or sham irradiated.

Figure 11. Experimental design and sample collection. $\boldsymbol{\nabla} / \mathbf{\Delta}$ indicates sample collection. Use of baseline samples and the crossover design allows subjects serve as their own controls. 86

Figure 12. Circulating levels of (A) hs-CRP, (B) IL-6 and (C) LBP in subjects prior to and after consumption of either $50 \mathrm{~g}$ (low dose) or $100 \mathrm{~g}$ (high dose) of sumac sorghum breakfast cereal each day.

Figure 13. Alpha diversity estimates at Pre-Low, Post-Low, Pre-High, and PostHigh sampling time points. No significant differences were observed between groups at any time point.

Figure 14. Beta-diversity principal components plot employing Bray Curtis dissimilarity for distance calculation between samples. Color coding by treatment: Pre1-red, Pre2-orange, Post High-blue, Post Low-green. No clustering by treatment was observed.

Figure 15. Relative concentrations of select plasma metabolites revealed by multiplatform mass spectrometry. A-E, metabolites generated from the microbial metabolism of dietary polyphenols and phenylalanine; $\mathrm{F}$, derived from microbial metabolism of tryptophan; G-H, gammaglutamyl amino acids involved in recycling oxidized glutathione. * indicates statistical analysis was performed on log transformed data. The treatment effect Q-value is also presented.

Figure 16. Conceptual diagram illustrating the production of metabolites from the dietary precursors: phenylalanine, proanthocyanidins, and chlorogenic acid. Adapted from Wikoff et al. and Gonthier et al. $(355,356)$. 100 


\section{LIST OF TABLES}

Table 1. Total number of $\mathrm{AC}^{1}, \mathrm{ACF}$, and $\mathrm{HMACF}$ were reduced in rats treated with AOM and fed a dried plum diet, versus basal diet counterparts.

Table 2. Colonocyte gene expression ${ }^{1}$ levels in rats treated with AOM, or saline, and fed a basal or dried plum diet.

Table 3. Percent abundance of dominant phyla in rats treated with AOM, or saline, and fed a basal or dried plum diet.

Table 4. Results of PERMANOVA analysis on unweighted beta-diversity principal coordinates data. 48

Table 5. Predicted microbial metagenome KEGG functional pathways (Level 2 pathway consolidation) significantly altered by diet in the proximal and distal colon of rats consuming a basal or dried plum diet and treated with AOM or saline.

Table 6. Select predicted microbial metagenome KEGG functional pathways (Level 3 pathway consolidation) significantly altered by diet in the proximal and distal colon of rats consuming a basal or dried plum diet and treated with AOM or saline.

Table 7. Rat fecal SCFA levels at 3 wk post-diet start, and $48 \mathrm{~h}$ and $8 \mathrm{wk}$ following the second AOM injection, organized by diet+injection group for each time point.

Table 8. Top metabolites affected by dried plum in the distal colon, their super pathway classification, database identifiers (if known), and fold of change (PD+A/BD+A, Least square means). Metabolites are sorted by increasing $Q$-values (i.e., FDR adjusted $P$-value). 55

Table 9. Fatty acid profiles* of basal and plum diets used in the experiment. 56

Table 10. Top canonical pathways detected by IPA software, number of compounds in the dataset related to the pathway, and their respective $P$ values.

Table 11. Alterations in bacterial phyla and genera in C57BL/6 mice consuming a control or dried plum diet and exposed to $1 \mathrm{~Gy}$ of HZE radiation or sham irradiated. 
Table 12. Colon luminal SCFA concentrations in male C57BL/6J mice provided a control diet (AIN93M) or a diet containing dried plum at $25 \%$ of total diet weight and irradiated with $1 \mathrm{~Gy}$ Fe ions $(\sim 10 \mathrm{cGy} / \mathrm{min}, 600 \mathrm{MeV})$ or sham irradiated.

Table 13. Baseline characteristics of all subjects $(n=24)$ and separated by gender.

Table 14. Quantitative SRM Transitions for compounds analyzed in fecal samples.

Table 15. Table of relevant literature and rationale supporting the investigation of specific metabolites in the human fecal samples.

Table 16. Data from 3-day diet records collected at baseline and at the end of each dietary intervention period.

Table 17. Blood glucose and lipid profiles prior to and after consuming the sumac cereal.

Table 18. Changes in select microbial abundances in response to sumac sorghum intervention.

Table 19. Baseline concentrations and changes in response to low, high and the average treatment response for total and individual SCFA.

Table 20. Metabolites selected for targeted quantification in subject fecal samples at baseline and post-high time points.

Table 21. Paired t-test results for the targeted fecal metabolite quantification at baseline and post-high time points.

Table 22. Significant correlations between plasma metabolites and microbes (genus level) in response to the low level (50 g/day) consumption of dietary sumac sorghum in human subjects.

Table 23. Significant correlations between plasma metabolites and microbes (genus level) in response to the high level (100 g/day) consumption of dietary sumac sorghum in human subjects. 


\section{CHAPTER I}

\section{INTRODUCTION AND LITERATURE REVIEW*}

\section{Introduction}

Colon cancer is associated with various risk factors including abdominal obesity, chronic inflammation, and mutagen exposure (2-6). The classically-defined hallmarks of cancer are: 1) self-sufficiency in growth signals, 2) insensitivity to growth-inhibitory signals, 3) evasion of apoptosis, 4) limitless replicative potential, 5) sustained angiogenesis, and 6) tissue invasion and metastasis $(7,8)$. More recently, two emerging hallmarks of cancer have been proposed: 1$)$ deregulated cellular energetics, and 2) avoidance of immune destruction (9). Indeed, cancer cells are known to exhibit altered metabolism (i.e., the Warburg effect) (10-12). In addition, tumorpromoting inflammation, and genome instability and mutation have been proposed as cancer enabling characteristics (9). Alterations in the epigenome, such as global hypomethylation and site-specific hypermethylation of tumor suppressor genes, regulate gene expression patterns and have been associated with colon carcinogenesis (13).

The fundamental model of sporadic cancer development involves three steps: initiation, promotion, and progression (14). Initiation refers to an irreversible mutation event in the DNA sequence following exposure to mutagens, such as ionizing radiation, aromatic amines, polycyclic hydrocarbons, and oxygen free radicals $(14,15)$. Promotion is characterized by alterations in cell apoptosis and proliferation that occur intrinsically in response to initiation

\footnotetext{
* Part of this chapter is reprinted with permission from Shaping functional gut microbiota using dietary bioactives to reduce colon cancer risk by Seidel DV, Azcarate-Peril MA, Chapkin RS, and Turner ND, 2019. Semin Cancer Biol. 2017 Oct;46:191204 by Elsevier.
} 
(e.g., overexpression of mutant $\mathrm{p} 53$ ) and/or to promoters that drive proliferation and/or inhibit apoptosis of initiated cells (14). Progression is characterized by structural changes in cell karyotype, further accumulation of genetic mutations, anaplasia, development of malignant neoplasms, as well as tumor invasion and metastatic growth $(14,16)$. Interestingly, cells within the primary tumor are known to harbor heterogeneous mutation profiles making targeted treatment strategies more difficult (16). Therefore, significant efforts are made to inhibit carcinogenesis at the initiation and promotion stages.

Colon epithelial cells exist in a mutualistic relationship with bacteria residing in the lumen. The gut microbiota is estimated to contain over 1,000 different 'species-level' phylotypes (17). One recent metagenomics study reported a microbial gene catalog of 3.3 million genes, which is $\sim 150$ times larger than the human gene complement (17-19). The microbial metagenome permits a repertoire of metabolic functions that supplement host metabolism, and the gut microbiota has been implicated in a number of physiological processes, including modulation of intestinal motility, regulation of luminal $\mathrm{pH}$, stimulation of immune function, and metabolism of undigested food (20). Dysbiosis refers to perturbations in microbial populations, and has been linked to obesity, cancer, and promotion of colonic inflammation (e.g., inflammatory bowel disease (IBD) and colitis) (3, 21-26). Inflammation can occur in response to a variety of biological insults, including infection by pathogens, immune sensitivity, autoimmunity, and exposure to ionizing radiation, and has been implicated in both sporadic colorectal cancer (CRC) and colitis-associated colon cancer (CAC) (3, 6, 27-30).

Diet is a major contributor to mammalian health and metabolism, and research has demonstrated that diet also contributes to the composition and metabolism of the gut microbiota $(31,32)$. Epidemiological studies have revealed that consumption of fruits, vegetables, and 
whole grains reduces CRC risk (33-35). The chemoprotective activity afforded by fruit, vegetable, and whole grain consumption is believed to be due to the fiber content, antioxidant compounds, and other bioactive compounds (i.e., extra-nutritional elements of food that can affect living tissue) $(33,34,36)$. Diets containing bioactive compounds that directly inhibit proliferation and promote apoptosis of transformed cells are promising strategies for the prevention of colon cancer. Additionally, certain food constituents can undergo metabolism by the microbiota to produce secondary bioactive compounds that then affect host physiology, such as the fermentation of dietary fiber into short-chain fatty acids (SCFA) (37-41).

\section{Literature Review}

$\underline{\text { Host and microbe-associated alterations as contributors to colon carcinogenesis }}$

\section{Epithelial perturbations and their outcomes}

Colonocytes serve several important functions including nutrient absorption, mucin secretion, and endocrine activity. The differentiated cell types performing these functions are derived from stem cell precursors that reside at the base of the intestinal crypt (42). To date, there has been extensive debate regarding the identity of stem cells within the colon crypt. However, the leucine-rich-repeat-containing G-protein-coupled receptor 5 positive $\left(\mathrm{Lgr} 5^{+}\right)$crypt base columnar cells are a strong candidate, as these cells undergo cell division daily and their daughter cells constitute the transit-amplifying crypt compartment (42). Furthermore, lineage tracing studies using an inducible Cre knock-in allele and the Rosa26-lacZ reporter strain have demonstrated restricted expression of $\mathrm{Lgr}^{+}$cells to the base of the crypt that can generate all epithelial lineages over a 60-day period $(42,43)$. Daughter cells derived from the stem cell population will continue to divide and eventually differentiate into mature colonocytes as they migrate away from the base of the intestinal crypt toward the intestinal lumen. Mature, senescent 
cells, which are lost at the luminal surface via sloughing or spontaneous apoptosis, are normally replaced at an equal rate by proliferating cells (44). Coordinated proliferation and migration of colonic epithelial cells are necessary for the maintenance of barrier function and epithelial restitution following an injury to the epithelial cells $(44,45)$.

Adult colon stem cells sustain self-renewal and are target cells for cancer-initiating mutations $(43,46)$. Tissue-specific mutation accumulation in colon stem cells resulting in alterations in differentiation/plasticity, and stem cell location/proliferation are generally believed to represent the earliest step towards colon tumorigenesis $(47,48)$. The onset of colon tumorigenesis is often driven by mutations in the Wnt signaling pathway (49-51). Specifically, adenomatous polyposis coli (APC) loss-of-function or $\beta$-catenin gain-of-function mutations result in stabilization of free $\beta$-catenin, which leads to aberrant Wnt signaling that can drive tumorigenesis (49-51). Importantly, mutational activation of the Wnt pathway in $\mathrm{Lgr}^{+}$cells gives rise to intestinal tumors with greater efficiency than other intestinal cell types (43). According to the cancer stem cell hypothesis, the population of cells that propagate tumor formation is self-renewing and multipotent (52). Thus, intestinal stem cells are considered the cell-of-origin of cancer because these cells already exhibit self-renewing capacity and multipotency $(42,43,53)$. In addition to the concept that cancer stem cells are the direct products of neoplastically transformed normal adult stem cells, there is also evidence that transit amplifying cells with mutant genomes can dedifferentiate and enter the stem cell state (54). Adding to the complexity, recent evidence indicates that multiple stem cell hierarchies exist in the intestine and that plasticity within stem cell hierarchies mediates cellular fate in response to extrinsic factors such as nutrition, inflammation, and physical stress signals (55-59). Interestingly, a role for diet in the regulation of intestinal stem cells has been reported (60-63). 
Although the expansion of initiated cells is required for tumor formation, previous work has shown cell proliferation to be a poorer predictor of tumor development than markers of differentiation and apoptosis (64). Furthermore, tissues with relatively higher rates of proliferation do not appear to exhibit a higher incidence of spontaneous tumor development (65). Apoptosis is a key regulator of physiological development and tissue homeostasis (66). To date, two major mechanisms of apoptosis induction in mammalian cells have been elucidated in detail. The extrinsic (i.e., death receptor) and intrinsic (i.e., mitochondrial) apoptosis pathways involve caspase activation cascades leading to controlled cell death (66). The extrinsic apoptosis pathway requires activation of membrane receptors of the tumor necrosis factor (TNF) receptor superfamily such as CD95 (APO-1/Fas) or TNF-related apoptosis-inducing ligand (TRAIL) receptors $(66,67)$. Activation of the intrinsic pathway, which is often dysregulated in colon cancer, involves mitochondrial outer membrane permeabilization (MOMP) and subsequent release of cytochrome $\mathrm{c}$ and other proteins from the mitochondria, triggering caspase- 3 activation through the formation of an apoptosome complex $(68,69)$. B-cell lymphoma-2 (Bcl-2) family proteins, among other factors, regulate the intrinsic mitochondrial apoptosis pathway and appear to dictate the sensitivity or resistance of the cell to apoptosis induction (69-71). Activation of the intrinsic pathway in response to DNA damage provides a mechanism whereby DNA damaged cells can be removed from the tissue, thus preventing the propagation of transformed cells or retention of mutated progenitor cells (69). Inhibition of apoptosis is an important mechanism of tumor promotion that can arise from genetic and epigenetic alterations (e.g., p53 loss-of-function mutations or $b c l-2$ promoter hypomethylation, respectively) and exposure to chemical promoters (72-75). Additional evidence of epigenetic alterations contributing to colon carcinogenesis is the hypermethylation of the DNA repair gene, human 
MutL homolog 1 ( $h M L H 1$ ), which has been linked to microsatellite instability (MSI) associated colon carcinogenesis (76). Therefore, dietary bioactives targeting the colonocyte epigenome are promising strategies for reprogramming aberrant processes associated with colon cancer, such as inhibition of apoptosis in transformed cells $(14,73,77)$.

The unique molecular and genetic features observed in normal and diseased tissues of the proximal and distal colon reveal differing susceptibilities and mechanisms for disease initiation. One study examining gene expression profiles carried out on healthy adult human biopsy samples revealed more than 1000 differentially expressed genes between the ascending and descending colon (78). The Cancer Genome Anatomy Project (CGAP) functional grouping of the differentially expressed genes revealed major pathways implicated in colon cancer including epidermal growth factor (EGF), transforming growth factor beta (TGF- $\beta$ ), Wnt, Ras, insulin, and integrin signaling (78). Differences in gene expression between the proximal and distal colon may result from the observed differential DNA methylation patterns in colonocytes (79). Studies comparing cancers of the proximal and distal colon have revealed numerous differences in molecular and clinicopathological features, which reflect different susceptibilities to neoplastic transformation, such as the frequency of V-Ki-Ras2 Kirsten Rat Sarcoma 2 Viral Oncogene Homolog (KRAS) and B-Raf Proto-Oncogene, Serine/Threonine Kinase (BRAF) mutations, and the prevalence of MSI (80-82). In response to dietary fiber and carcinogen treatment, differences in colonocyte proliferation and differentiation between the proximal and distal rat colon have been observed (83). Furthermore, it is well documented that the gut microbiome is spatially heterogeneous, forming discrete microbial communities throughout the intestinal tract that differentially affect the host physiology (84). Research into the unique characteristics of these sites is necessary to fully appreciate the opportunities to mitigate colon carcinogenesis. 
As mentioned previously, inflammation is a known promoter of sporadic colon cancer and CAC. Although the cause of onset remains unknown, CAC is characterized by elevated reactive oxygen species (ROS), increases in pro-inflammatory cytokines and elevated expression of transcription factors involved in inflammation signaling (3). This chronic inflammation can lead to genome instability and mutations in critical regulatory genes, such as the tumor suppressor gene, $p 53$, that can drive carcinogenesis $(27,72,85)$. For example, we have recently demonstrated that loss of p53 function in stem cells enables colonic tumor formation only when combined with DNA damage and chronic inflammation (53). In sporadic CRC, inflammation is often a result of immune cell migration to the tumor site where activated immune cells produce ROS, reactive nitrogen intermediates, and pro-inflammatory cytokines, which can further damage DNA and drive cell proliferation $(3,30,86)$. Additionally, there is evidence linking inflammation to carcinogenesis via epigenetic modifications at critical regulatory gene loci (8790). It is apparent that inflammation can impact all stages of colon cancer development, and nutritional strategies aimed at modulating inflammation and ROS production may help mitigate disease risk.

\section{Changes to microbiome/metabolome and their role in tumorigenesis}

Currently, investigations into the physiological mechanisms by which the microbiota can influence colon cancer risk are limited due to the diversity and complexity of the microbiota. Despite the ongoing discussion concerning whether changes in the microbiota occur prior to or as a result of colon carcinogenesis, some significant observations have been made that suggest a causal role of the microbiota in the disease process. For example, studies employing rodent models of spontaneous, chemically-induced (e.g., azoxymethane, AOM), or genetically predisposed (e.g., $\mathrm{APC}^{\mathrm{min} /+}$ ) colon cancer demonstrate enhanced tumorigenesis in conventionally 
raised animals versus germ-free or antibiotic-treated animals, suggesting a promotive effect of the microbiota on tumor formation (91-97). Often, the enhanced tumorigenicity in conventionally raised mice is attributed to microbially-sustained levels of chronic low-grade inflammation that acts as a tumor promoter. However, activation of the innate immune system through toll-like receptor (TLR) and NOD-like receptor (NLR) agonists has been shown to enhance anti-tumor activity of the innate immune system $(98,99)$. Taken together, these observations suggest a double-edged role of the microbiota in colon carcinogenesis that may be dependent on the degree and mechanism of the innate immune response activation.

Because inflammation is a known driver of CRC, work has been conducted to better understand how the microbiota are altered by, or contribute to, pro-inflammatory states. A recent metagenomics study revealed that the fecal microbiome of patients with IBD has, on average, $25 \%$ fewer microbial genes than individuals not suffering from IBD (19). These findings are in agreement with other studies documenting a reduction in gut microbe diversity in association with IBD (100-102). An experimental animal model of IBD has shown that increases in colonic injury are negatively associated with Firmicutes, Actinobacteria, Lactobacillales and Lactobacillus (103). Dysbiosis has also been observed following spaceflight and radiation exposure in rodent models demonstrating the susceptibility of the microbiota to environmental factors other than the host diet (104). These observations suggest that microbial dysbiosis may act as a driver and/or may be a consequence of colon disease development.

Fecal profiling experiments of CRC patients and healthy subjects have revealed numerous taxonomic differences in the microbiota and these findings should be useful in directing mechanistic studies. The 454 pyrosequencing of fecal samples from $46 \mathrm{CRC}$ patients and 56 healthy volunteers identified 48 operational taxonomic units (OTUs) associated with the 
segregation of normal and CRC samples by UniFrac analysis (105). OTUs belonging to the genera Enterococcus, Escherichia/Shigella, Klebsiella, Streptococcus, and Peptostreptococcus were significantly more abundant in CRC patients. Conversely, OTUs related to the genus Roseburia and other butyrate-producing members of Lachnospiraceae were less abundant in CRC patients. In addition, quantitative polymerase chain reaction (qPCR) analysis of microbial butyrate synthesis genes confirmed lower levels of butyrate-producing bacteria in CRC patients, supporting the identity of butyrate-producing microbes as contributors to intestinal health. These observations were similarly replicated in a more recent study that found nine OTUs represented by the butyrate-producing genera Faecalibacterium and Roseburia to be significantly less abundant in CRC patients versus healthy control subjects (106). Another study revealed a greater presence of Fusobacterium nucleatum and Enterobacteriaceae in CRC patients versus healthy control subjects (107).

In addition to overall changes in the gut microbiota of CRC patients versus healthy controls, there is evidence demonstrating microbial differences between normal and diseased tissue sites within a given individual. One study characterizing the microbial structure of the intestinal lumen, cancerous tissue, and matched noncancerous normal tissue, revealed numerous differences between these sampling sites (108). Microbial diversity was significantly lower in tumor tissue than noncancerous tissue, suggesting a more selective microenvironment exists in proximity to diseased tissue. In cancerous tissue, Lactobacillales was enriched, whereas Faecalibacterium was reduced. With respect to mucosa-adherent bacteria, Bifidobacterium, Faecalibacterium, and Blautia were reduced in CRC patients whereas Fusobacterium, Porphyromonas, Peptostreptococcus, and Mogibacterium were increased. Lastly, in the lumen, bacteria associated with metabolic disorders or metabolic exchange with the host, such as 
Erysipelotrichaceae, Prevotellaceae, and Coriobacteriaceae, were increased in CRC patient samples. In another study, a lower abundance of Staphylococcus and Bacillus and a higher abundance of Escherichia-Shigella and Prevotella were observed in polyp-derived mucosal samples compared to adjacent healthy tissue biopsies (107). Altogether, these findings suggest certain bacteria may better compete in the transformed niche and that characterizing these microenvironments could reveal novel mechanisms by which the microbiota influence disease progression.

Metabolomics analysis of CRC patients versus healthy controls is a useful tool for investigating alterations in co-metabolism or metabolic exchange between host cells and the gut microbiota and permits identification of novel CRC metabolite biomarkers. Studies of the fecal metabolome have revealed alterations in SCFA, amino acid (protein), and unsaturated fatty acid metabolism in CRC patients (109). Another study using serum samples from colorectal adenoma, CRC, or healthy control subjects, revealed pathways for urea, caffeine, and galactose metabolism were related to CRC progression (110). Similar to microbial observations between normal and diseased tissue, metabolomics analyses have revealed different metabolite signatures between stool, cancers, and healthy adjacent mucosa (111). In that study, metabolic pathway analysis of significantly different metabolites revealed aberrant SCFA metabolism, fructose, mannose, and galactose metabolism, and glycolytic, gluconeogenic, and pyruvate metabolism in CRC tissue of 17 CRC patients, versus healthy adjacent tissue. Furthermore, whereas nearly all of the metabolites detected in CRC tissue were found in adjacent mucosa, less than 50\% of metabolites (213 out of 500 total metabolites) detected in the stool were shared with either tissue site. These findings reinforce the need to consider metabolomic profiles derived from multiple adjacent 
sampling sites to enhance biomarker discovery and personalized treatment and prevention strategies.

To define a beneficial microbiota composition that is capable of suppressing CRC, it will be important to first understand the mechanisms by which the microbiota can predispose host cells to carcinogenesis. Some bacteria have been shown to directly initiate carcinogenesis via the production of toxins that can damage DNA (112). For example, colibactin toxin produced by $E$. coli belonging to the B2 phylogroup causes DNA crosslinks and double strand breaks (DSB) that can lead to mutations (113). Bacteroides fragilis toxin is known to activate Wnt and NF- $\mathrm{kB}$ signaling pathways, increase cell proliferation, enhance epithelial release of pro-inflammatory molecules, and induce DNA damage in vitro (114-116). More recently, the gene encoding Bacteroides fragilis toxin was found to be more abundant in tumor mucosa samples than control biopsies, especially in late-stage CRC, suggesting that the bacteria capable of synthesizing the toxin play a persistent role in promoting tumor growth through sustained activation of tumorpromoting pathways (117). The Salmonella protein AvrA has recently been shown to activate the STAT3 pathway in a CAC mouse model (AOM plus dextran sulfate sodium, DSS) (118). The role of the STAT3 pathway and signaling in colon cancer has been well established (119-122). Indirect effects of specific bacteria on carcinogenesis have been characterized that are largely dependent on the immune system for manifestation. In addition to inducing DNA modifications, the $E$. coli strain that produces colibactin has recently been shown to encourage pro-tumoral activities of tumor-associated macrophages by infecting and persisting within the immune cells. As a result, these bacteria induce sustained cyclooxygenase-2 (COX-2) expression by macrophages, which is a hallmark of the inflammation associated with colon cancer (123). A recently proposed mechanism for bacterial-induced chromosomal instability (CIN) was reported 
by Wang and Huycke (124) in which macrophage polarization to an M1 phenotype by commensal bacteria leads to a trans-4-hydroxy-2-nonenol (4-HNE) mediated bystander effect in colon epithelial cells. The 4-HNE released by M1 macrophages in response to commensal invasion during periods of intestinal barrier dysfunction causes mutations, DSB, and spindle dysfunction leading to CIN in epithelial cells. Taken together, these experiments demonstrate the potential of direct and indirect mechanisms of the gut microbiota to initiate and promote colon carcinogenesis.

\section{Radiation effects on colon physiology and microbiota}

Ionizing radiation (IR) is a form of radiation which is capable of removing electrons from atoms and can take the form of particles (e.g., electrons, protons, neutrons, heavy charged particles, etc.) or electromagnetic waves (e.g. x-rays and gamma rays) (125). Linear energy transfer (LET) refers to the energy transferred from the radiation source to the medium per unit length of the track (125). In radiation biology, the medium is the tissue or cells that the particle traverses as it deposits energy. In general, electromagnetic radiation is considered low LET and particulate radiation is considered high LET (125). High LET radiation is densely ionizing and the DNA damage it creates is more difficult to repair than sparsely ionizing low LET radiation (125). In space, astronauts are exposed to ionizing radiation emitted from the Sun (i.e., solar radiation) and from other celestial bodies (i.e., galactic cosmic radiation; GCR) (126). Solar radiation is mostly protons and electrons of lower energy than GCR, which is composed mostly of high energy protons (83\%), alpha particles (14\%), high charge, high energy ions (HZE, 1\%), and electrons (2\%) (126). Both particulate and electromagnetic ionizing radiation can damage DNA directly by ionizing atoms in the DNA molecule (125). Alternatively, ionizing radiation can cause indirect DNA damage via the generation of reactive oxygen species (ROS) such as 
superoxide $\left(\mathrm{O}_{2}^{-}\right)$and hydroxyl radicals $\left(\mathrm{OH}^{-}\right)$, which can interact with the DNA molecule (125, 127). This DNA damage can manifest as base pair loss, base modification, single or double strand breaks, cross-links, and chromosomal aberrations (125). Following DNA damage recognition, a cell may undergo apoptosis immediately, or may initiate p53-dependent cell cycle arrest in order to repair the damage (128). If the repair process is successful, the cell may continue as normal; however, incorrect repair can lead to mutations, chromosome aberrations, and/or genomic instability, which can lead to cell death or fixation of the mutation in the genome (128). Thus, the decision between life and death for the cell is related to the complexity of the DNA damage, the fidelity of the repair process, the ratio of proteins involved in the regulation of apoptosis (e.g., bax, bcl-2, etc.), and the presence of survival and death signals (128). In summary, DNA damaged cells that avoid apoptosis, or fail to correctly repair the damage, can develop tumor-initiating mutations that lead to carcinogenesis.

A recent estimate for the shortest round trip to Mars (180 days without surface time) indicates an equivalent dose of 0.66 Sievert (Sv) (129). To put this into perspective, the current career limit imposed on astronauts is $1 \mathrm{~Sv}$, which corresponds to an increased risk of fatal cancer of approximately 5.5\% (129). The most recent estimates for probability of causation for cancer risk indicate the colon to be the third most susceptible organ-site to tumorigenesis following exposure to GCR (130).

Microbial dysbiosis has been observed following spaceflight and radiation exposure in rodent models (104). These observations suggest that microbial dysbiosis may act as a driver and/or a consequence of disease development in these experimental conditions. Therefore, understanding how diet and other environmental factors, such as radiation exposure, can affect the microbiota and host-microbe interaction is a critical element of colon cancer prevention. 
Dietary interventions aimed at modulating dysbiosis directly (i.e., probiotics) or by promoting the growth of beneficial bacteria (i.e., prebiotics) may be advantageous for at risk populations, such as astronauts.

\section{Dietary mediators of colon tumorigenesis}

A recent review points to the importance of many different diet-derived biologically active compounds in the inhibition of carcinogenesis (8). Dietary compounds may reach the colon because they are: 1) too large to be absorbed in the small intestine, 2) bioavailable compounds that escape deglycosylation and absorption in the small intestine, or 3) aren't accessible to the host due to their intercalation in the food matrix (40). Those compounds absorbed in the small intestine, conjugated by the liver, and returned to the intestine via enterohepatic circulation also may reach the large intestine (40). Additionally, there is research to suggest that processing bioactive-containing foodstuffs can affect the bioaccessibility of bioactives, which may result in some molecules reaching the colon that otherwise might have been absorbed in the small intestine $(131,132)$.

Dietary bioactives have the potential to directly modify and/or mitigate tumorigenic processes via multiple pathways. These pathways may be microbe-independent (i.e., direct) or microbe-dependent (i.e., indirect), or both. Microbe-independent bioactivity refers to actions of the non-metabolized bioactive compounds directly on host cells and tissues, which we would anticipate also observing in a germ-free animal. Alternatively, microbe-dependent pathways would depend on the metabolism of the native bioactive compound by the microbiota into secondary metabolites that then exert some bioactive function. Furthermore, the microbedependent process could refer to diet-induced modifications to the number, composition, and/or functional profile of the colonic microbiota that, in turn, alters the production of microbial 
metabolites from non-bioactive substrates (e.g., bioactive compounds selecting for microbes that more effectively generate SCFA from dietary fiber) (133). Indeed, some dietary components exert bactericidal functions and are, therefore, direct modifiers of the gut microbiota (134-136). Similar to the observed differences in physiology and cancer phenotype/genotype between the proximal and distal colon mentioned previously, the composition of the microbiota is also known to differ spatially along the colorectal axis and between mucosal and luminal sites $(18,137,138)$. The observed differences between lumen- and mucosa-associated microbial communities likely reflect the nature of the host-microbe interaction for these sub-populations and importantly, the availability of their preferred substrates.

The most often ascribed function of dietary bioactives, particularly for polyphenolics, is their antioxidant capacity, and studies have estimated that $90-95 \%$ of dietary polyphenols escape absorption in the small intestine due to their size (139). The gut microbiota are capable of a variety of metabolic reactions that affect the structure and functional groupings of bioactives including ring-C cleavage, dehydroxylation, decarboxylation, deglycosylation, and demethylation (40). Indeed, there is substantial evidence that much of the biological response to dietary bioactives is due to microbial derivatives rather than the native compounds (140-143). A large consortium of authors reviewing the potential of bioactive compounds in cancer inhibition stressed the importance of exposure to these molecules during the early stages of cell transformation (8). They noted their efficacy is greatest during this time when most of the cellular changes are likely to be epigenetic in nature.

\section{Fiber and prebiotics}

Nondigestible carbohydrates (i.e., dietary fiber) pass through the small intestine into the cecum and large intestine where they undergo metabolism by the colonic microbiota (144). 
Many bacteria preferentially metabolize carbohydrates over other energy sources such as protein (145). Predominantly saccharolytic fermentation occurs in the cecum and proximal colon of humans and rodents, where most SCFA production takes place (146-148). The metabolic fate of fiber depends largely on the solubility/fermentability of the fiber itself and the microbiota present in the colon during digestion (149). Readily fermentable fibers such as oat bran, pectin, and guar are easily fermented by the microbiota to produce methane, carbon dioxide, and SCFA, whereas cellulose and wheat bran are poorly fermented $(149,150)$. Acetate, propionate, and butyrate are the most abundant SCFA in the colon, and concentrations typically decrease from the proximal to the distal colon $(18,80)$. Benefits of consuming both readily fermentable and poorly fermentable fibers have been established, with the latter being due to its hydrophilic bulking activity, which serves to dilute carcinogens, pro-carcinogens, and other potential tumor promoters in the intestinal lumen (151). Furthermore, stool-bulking agents have also been shown to increase the rate of digesta passage through the intestine, which can both minimize exposure to toxic compounds in the lumen and increase the levels of butyrate in the distal colon (152). In rats exposed to AOM, consumption of a wheat bran diet significantly lowered tumor incidence compared to animals consuming an oat bran diet (153). In addition, consumption of the wheat bran diet was associated with lower body weight, more normalized ratios of SCFA in the intestinal lumen, and increased fecal mass and bulk, which suggests increased bulking ability and dilution potential of wheat bran versus the more readily fermentable oat bran diet.

If dietary fiber and resistant carbohydrates are limiting, the gut microbiota in the distal colon will need to rely on proteolytic fermentation to meet their energy needs (145). Proteolytic fermentation results in SCFA, along with a number of putatively toxic metabolites such as ammonia and sulphur-containing compounds (145). Dietary protein levels may also influence 
butyrate utilization as a high protein diet has been found to decrease expression of the proton coupled monocarboxylate transporter 1 (MCT1, a butyrate transporter) in the colon of piglets (154). In contrast, Liu et al. found no effect of dietary protein on MCT1 expression or luminal butyrate levels in rats (155).

Butyrate is a four carbon SCFA and the preferred energy substrate of colonocytes (156). In healthy humans, absolute concentrations of butyrate range from 11 to $25 \mathrm{mM}$ in the feces (148). Bacteria synthesize butyrate from butyryl-coenzyme A (CoA) derived from acetyl-CoA using one of two enzymes, butyrate kinase or butyryl-CoA:acetate-CoA transferase (157). Butyrate is absorbed by colonocytes via passive diffusion and by active transport with various ion exchange transporters, such as the sodium-coupled monocarboxylate transporter 1 (Slc5a8) and MCT1 (also known as Slc16a1) (148). Butyrate has been intensively studied for its ability to inhibit inflammation and carcinogenesis, reduce oxidative stress, and promote colonic barrier function (148). Butyrate has also been shown to affect gene expression via epigenetic modification of chromatin. In cancer cells exhibiting the Warburg effect, butyrate accumulates in the cell and inhibits histone deacetylase (HDAC) activity and stimulates histone acetyltransferase (HAT) activity by acting as an acetyl-CoA donor, thereby regulating genes that enhance apoptosis and reduce cell proliferation $(156,158)$. Our lab has demonstrated increased $b c l-2$ promoter methylation and levels of apoptosis in rats consuming a fish oil and pectin diet versus animals consuming a diet containing corn oil and cellulose (73). Additional in vitro experiments demonstrate butyrate's ability to reduce global and Bcl-2-like protein 11 (BCL2L11) promoter methylation in HCT-116 colon cancer cells (77). These data support the identity of butyrate as a modifier of the epigenome and promoter of apoptosis in colon cancer cells. Colonic mucosa from slc $5 a 8^{-/}$knockout mice exhibits dramatically increased expression of inducible nitric oxide 
synthase (iNOS), a marker of inflammation, in addition to genes of the interferon gamma (IFN- $\gamma$ ) and TGF- $\beta$ signaling pathways compared to wild-type mice (159). Indeed, Slc5a8 plays a critical role in the ability of butyrate to suppress colon inflammation and cancer (160-162). Additionally, butyrate was shown to suppress colonic inflammation by two mechanisms: 1) inhibition of STAT1 phosphorylation by IFN- $\gamma$ in colonic epithelial cells leading to a reduction in iNOS expression, and 2) inhibition of Fas promoter-bound HDAC1 leading to increased T cell expression of Fas and, thus, enhanced sensitivity to apoptosis of activated T cells thereby reducing T cell production of IFN- $\gamma$ (159). Butyrate has also been shown to induce apoptosis via a non-mitochondrial, Fas-mediated, extrinsic pathway in colonocytes $(163,164)$.

Research has also demonstrated the ability of SCFA to affect immune function. Kim et al. recently demonstrated that SCFA impact the metabolism of B lymphocytes by increasing acetylCoA levels, oxidative phosphorylation, glycolysis, and fatty acid synthesis, and that these changes in metabolism support the production of antibodies necessary for preventing infection by pathogens (165). SCFA were also found to regulate gene expression programs needed for plasma B cell differentiation. Importantly, dietary fiber and SCFA both increased intestinal (Immunoglobulin A, $\operatorname{Ig} \mathrm{A}$ ) and systemic (IgA and $\operatorname{IgG}$ ) antibody levels supporting the notion that the gut microbiota, by way of metabolism of dietary fiber, promotes systemic immunity. The gut microbiota and its metabolites (SCFA, particularly butyrate) have also been shown to affect the differentiation of naïve $\mathrm{T}$ cells in the gut epithelium (166). The mechanisms responsible for the differentiation of naïve $\mathrm{T}$ cells into Treg cells include direct actions of butyrate on naïve $\mathrm{T}$ cells (i.e., histone $\mathrm{H} 3$ acetylation at the Foxp3 locus and Gpr109a activation) and indirect actions via changes in epithelial cell cytokine production (e.g., TGF- $\beta)$. 
Taken together, these observations support a pleiotropic role of butyrate on the colon epithelium as a modulator of inflammation and colonocyte physiology. Interestingly, there is some evidence that butyrate may promote colon tumorigenesis (167-169) or that some dietary interventions reduced colon carcinogenesis in a manner not related to fecal butyrate levels (153). These findings suggest that microbial metabolites such as short chain fatty acids contribute to colon cancer risk in a complex, context-dependent manner. Additional work needs to be conducted to better understand how gut microbial metabolites impact gut health. Specifically, understanding how other dietary components may interact with microbial metabolite production and their effects on host tissue should be explored if we are to identify dietary patterns capable of suppressing colon carcinogenesis.

\section{Phenolic bioactives and other small bioactive compounds}

Most fruits and vegetables contain bioactive compounds. The phytochemical profile can vary between different varieties of the same food and can be influenced by how the foods are processed (170-172). The concentration of phytochemicals also varies throughout the fruit (e.g. seed, peel, pulp) and is typically highest in the fruit peel where the compounds function as antioxidants to prevent damage from ultraviolet radiation $(173,174)$. Many phytochemicals are known to have anti-microbial and anti-inflammatory properties (175-177). The mechanisms by which the compounds exert these effects in vivo and to what extent they are absorbed and metabolized by the host and gut microbiota remains to be fully characterized. For example, polyphenols are a structural class of phytochemicals with multiple phenolic units found naturally in fruits, vegetables, and whole grains (178-181). Numerous studies have revealed chemoprotective effects of polyphenolic compounds in vivo and in vitro (182-185). Additionally, there is evidence that polyphenols may exert their chemoprotective effects through epigenetic 
mechanisms (186-188). The bioavailability of polyphenols varies depending on the individual compound; however, it has been shown that human colonic bacteria can metabolize polyphenols into lower-molecular-weight bioactive compounds, which may be more bioavailable to the host $(141,185,189,190)$. Further, consumption of dietary polyphenols is known to alter the composition of the gut microbiota, which can affect host health (191-193). Understanding how foods containing dietary polyphenols can shape the microbiota composition and metabolism will be important for defining bioactive profiles. Likewise, characterizing the metabolites generated from microbial metabolism of polyphenols, and their presence in the intestinal lumen and systemic circulation will be important for future studies seeking to elucidate biomolecular mechanisms.

Because a whole food (e.g., fruit or vegetable) and combinations of foods contain a mixture of bioactive compounds in combination with the micro and macronutrients of the food, it will be important to characterize how compounds synergize in vivo. Within a whole food, such as prunes (dried plums), there are a variety of bioactive compounds including phenolic acids (e.g., quercetin, chlorogenic acid, and neochlorogenic acid), sugar alcohols (e.g., sorbitol and mannitol), and hydroxycinnamic acids (e.g., sinapinic acid), as well as a mixture of soluble and insoluble fiber $(194,195)$. Prune consumption is commonly associated with a reduction in constipation and improved intestinal motility (194). These effects can be attributed in part to the dietary fiber found in prunes; however, they are also noted when consuming prune juice, which lacks the insoluble fiber found in the whole fruit. Hence, not all of the bioactive properties ascribed to prune consumption are due to its fiber content. Our lab has shown that quercetin and chlorogenic acid influence fecal SCFA concentrations, suggesting alterations in the gut microbiota or in the absorption of SCFA by the host (196). Furthermore, our lab has 
demonstrated the ability of quercetin to reduce the numbers of high multiplicity aberrant crypt foci (HMACF), which are thought to be most indicative of eventual tumor formation $(197,198)$. A reduction in proliferation and an increase in apoptosis were also observed in rats fed quercetin, and these effects are thought to be related to observed reductions in pro-inflammatory mediators, cyclooxygenase 1 and 2 (COX-1 and COX-2) (198), as well as through impacts on other signaling pathways including NF-אB, PI3K/Akt/mTOR, and JNK/JUN (198-201). Quercetin has also been shown to reduce the incidence, multiplicity, and size of colorectal tumors in male F344 rats treated with AOM, compared to control diet animals (202). Sorbitol is a sugar alcohol found in high concentrations in dried plums and contributes to the laxative effect associated with prune consumption (203). Sorbitol has been shown to induce apoptosis in HCT-116 cancer cells via activation of the p38 mitogen-activated protein kinase (MAPK) pathway, which activates the mitochondrial death cascade (204). Additionally, in rats consuming a dried plum powder, analysis of the microbial metagenome revealed a reduction in the relative abundance of microbial genes responsible for the synthesis of secondary bile acids, which are known to induce DNA damage and promote colon cancer (205-207).

Another major source of dietary phytochemicals is whole grains $(179,208)$. For example, sorghum is an ancient grain predominantly grown in dry, arid zones. Sorghum varieties contain different levels and types of phenolic and polyphenolic compounds (179). Sumac sorghum contains high levels of condensed tannins (i.e., polymerized flavonols), which contribute to its high phenolic content and oxygen radical absorbance capacity (179). Indeed, sumac sorghum has been characterized as having greater antioxidant capacity than many other grains, blueberries, and pomegranates $(209,210)$. Decortication of the sorghum grain to produce sorghum bran increases the concentration (3-6 times higher than the whole grain) of the phenolic compounds 
(210). Our lab has previously shown that polyphenol-rich sorghum brans alter the microbial composition and beneficially affect microbial diversity and richness in a rat model of colitis (103). In addition, we have shown that less soluble fibers with lower fermentability, such as sorghum bran and cellulose, are more protective against colonic injury during bouts of colitis than highly fermentable fibers, such as pectin (211). Although these findings are in contrast to the previously discussed protective effects of soluble fibers during carcinogenesis, it is evident that fibers of varying fermentability/solubility exert their effects on the colonic mucosa in a disease-dependent context that likely depends on the composition of the gut microbiota. Furthermore, our lab has demonstrated that diets containing sumac bran alter fecal SCFA levels, suggesting these polyphenol-rich fiber sources may alter microbial metabolism or host SCFA absorption (212).

As previously mentioned, it will be important to characterize the mechanisms by which dietary bioactives exert their chemoprotective activities in order to better understand how specific phytochemicals can prevent or inhibit specific diseases. This includes identifying and characterizing receptors for native phytochemicals and their microbial metabolites. Integrating microbiome, metabolome, and gene expression/pathology data

The ultimate goal for microbial community profiling is to accurately assess which microbes are present in a sample and their collective genomes (metagenomics), the genes being transcribed and proteins translated (metatranscriptomics and metaproteomics, respectively), and the metabolites present (metabolomics). Indeed, the composition of bacteria between individuals, in terms of taxonomic identity, tends to be more unstable than the functional profile of a microbiome sample (213-215). A recent study employing systematic comparison of metagenomic and metatranscriptomics data from healthy human subjects demonstrated that $41 \%$ 
of microbial gene transcripts were not differentially regulated relative to their genomic abundance and that the microbial transcriptome profile was significantly more individualized than the metagenome profile (223). Metabolome profiling enables the relative assessment of thousands of metabolites including microbe-, diet-, and host-derived molecules and represents an instantaneous index of the combined cellular processes taking place in the sample. However, because of the complexity of system being sampled, it will be difficult to attribute the production of specific metabolites with specific bacteria, especially when the genes responsible for encoding a given function are conserved across species. More work will be needed to determine which level of information provides the best opportunity to study specific physiological and ecological states.

The challenge of integrating multiple 'omics' profiles, such as microbe and host functions occurring simultaneously, was a goal of the second phase of the Human Microbiome Project (HMP), termed the Integrative Human Microbiome Project (iHMP) (216). The consolidation of data from these and other similar projects into in silico networks, will allow the study of the dynamic interactions occurring between the host and the microbiome during various disease states to identify causal bio-molecular mechanisms for targeted study. For example, research conducted by Sridharan et al. used in silico microbial metabolism reaction networks coupled with two independent mass spectrometry metabolomics analyses to identify and characterize microbial metabolites which were then used in vitro to determine mechanisms of action (224).

Early investigations to characterize and profile the microbiome utilized culture methods and microscopy, but these efforts were limited by cultivability, nonspecific substrate utilization, and the inability to distinguish morphologically similar bacteria from one another (217). Early 
next-generation-sequencing techniques enabled the classification of bacteria from a sample at the taxonomic level by sequencing variable regions of the $16 \mathrm{~S}$ ribosomal RNA (rRNA) gene. Taxonomic microbial profiling from $16 \mathrm{~S}$ rRNA sequencing yields relative abundances of bacteria classified into OTUs, based on sequence similarity of sample reads to known bacterial sequences (218). These data can be used to estimate diversity within and between samples based on the number of species in a sample (richness) and their relative abundance (evenness) (219). However, marker gene surveys such as $16 \mathrm{~S}$ sequencing only permit the identification of bacteria present in a given sample and provide no information about the functional gene content of the microbiome sample as a whole, which is often argued to be substantially more informative (215). This information can be obtained using deep metagenome shotgun sequencing, but is much costlier and computationally intensive than marker gene survey sequencing. Therefore, strategies have been designed to use marker gene survey data to predict the functional gene content (i.e., the metagenome) of a microbiome sample using the same input data (220). Phylogenetic Investigation of Communities by Reconstruction of Unobserved States (PICRUSt) is one such tool that predicts the metagenome information (i.e., gene counts) of a $16 \mathrm{~S}$ marker gene survey data set, which are then collapsed into KEGG pathways (220). In traditional metagenome sequencing, sequences are aligned to a gene catalog and the functional potential assessed by mapping genes to a KEGG database or through gene ontology (GO) enrichment analysis (221, 222).

Despite the overall reduction in colon cancer incidence, the increase in distal cancer incidence in individuals younger than the previously recommended initial screening age (50 years old) supports the need for earlier screening (223). Recently, the American Cancer Society recommended the screening age for CRC be lowered to 45 years for average risk individuals. 
This recommendation is likely driven by CRC data indicating individuals aged $\leq 50$ years old with CRC often present with more advanced cancers and a concomitant higher risk of mortality (1). Earlier colon screening coupled with fecal microbial profiling to identify cancer-associated microbes would enhance colon cancer prevention and treatment. It is estimated that the transition from precancerous cell to malignant colorectal tumor takes $\sim 10-15$ years implicating a substantial window for pre-cancer screening and implementation of prevention techniques (224). As critical bioinformatics approaches become available for the integration of 'multi-omics' analyses and as the required sample input continues to decrease it will be extremely beneficial to adopt more precise sampling procedures for characterizing the microbiota and interactions within a given microenvironment. The resolution afforded by passive fecal collection is useful as a clinical diagnostic test for cancer associated microbes; however, patients already diagnosed with colon cancer or found to have polyps following colonoscopy could benefit from more precise diagnostic protocols. Similar to the procedures used for genotyping a tumor biopsy to inform targeted therapeutic strategies, targeted tumor- or polyp-associated microbial profiling could reveal additional host-microbe signaling networks that can be targeted for therapy.

Distinguishing between host- and microbial-derived growth signals could help explain the discrepancy in treatment responses of CRC patients. Although challenging, identifying critical microbial biomarkers should improve screening and early disease detection (168). For example, the discovery of hydrogen sulfide producing bacteria (i.e., sulfidogenic bacteria) and its contribution to inflammation and colorectal cancer (31, 225-227). Dietary bioactives can then be used to select against (or promote the growth of) specific bacteria (versus broad-spectrum antibiotics), can inhibit signaling pathways downstream of microbial activities, and/or can 
inactivate harmful byproducts of metabolism, which will improve the usefulness of diagnostic fecal microbiome profiling.

Nutrigenomics and nutrigenetics are 'multi-omics' manifestations aimed at understanding nutrient-gene interactions. Indeed, diet can function at multiple levels to regulate the flow of genetic information (228-231). These sciences and the knowledge acquired from them will be necessary for the development of personalized nutrition and disease prevention/treatment strategies, many of which are already used to some degree for the treatment of diet-related diseases, such as inflammatory bowel disease, obesity, diabetes, and cancer (232). Understanding how and to what extent specific nutrients affect the expression of microbial genes adds an additional layer of complexity due to the considerable microbial variation between individuals and the ostensibly infinite number of combinations between host and microbial genomes. Presumably, however, better health outcomes from nutritional interventions would be achieved by taking into consideration an individual's inherited and acquired genetic characteristics, age, dietary and lifestyle preferences, and gut microbiome profile.

Our understanding of the capacity of the gut microbiota to affect host physiology continues to increase and reinforces the necessity to study modifiers of the gut microbiome. It is irrefutable that the gut microbiota plays a critical role in regulating the intestinal epithelium and that the consequences of alterations in the gut microbiome can extend beyond the intestines. The concept that the gut microbiota can impact the development and activity of the host immune system, that the host immune system is a governor of the gut microbiota, and that microbial profiles are far more individualized than originally perceived, underscores the necessity to understand how individual genetic polymorphisms can personalize the gut microbiota and, consequently, the ability of diet to influence these processes. The fact that the diet can shape the 
composition and function of the gut microbiota and that the microbiota can influence the health of the host beyond the intestinal epithelium implies a third mechanism by which diet can promote intestinal health; physiological changes in response to microbially-derived metabolites or ligands in other tissue sites (e.g., liver, muscle, etc.) may provide feedback to the intestinal epithelium that affects colon cancer risk and the risk of other associated diseases. Recent evidence suggesting a role of the gut microbiota in modulating host satiety and brain function (i.e., gut-brain axis) is remarkably fascinating and further emphasizes the need for understanding the capacity for diet to shape gut microbiota function and metabolism (233-237).

Despite the increasing acceptance of this paradigm, causative roles for specific bacteria are only suggested by current research. Experiments attempting to define the direct contribution of specific bacterium (or bacteria) to colon health/disease will require tightly controlled animal studies utilizing well-characterized dietary, environmental and disease conditions. Data produced by these studies may be more generalizable to human populations. Although fecal bacteria profiling has not yet been employed as a clinical tool for CRC diagnosis, research into this area of noninvasive diagnosis shows promise (238). Similarly, despite changes in the microbiota being associated with disease, there are no clear bacterial biomarkers of colorectal cancer; however, elucidation of microbial pathways involved in the synthesis or detoxification of genotoxic agents (e.g., colonic sulfur metabolism, secondary bile acid production, etc.) will enable identification of novel compounds and biomarkers for this purpose. With respect to colon health, the gut microbiota influences cellular processes by internal and external mechanisms, which include receptor binding and signal transduction/inhibition, modulation of gene and protein expression, epigenetic modifications, changes in metabolism, and cell differentiation/fate. Furthermore, dietary phytochemicals that affect the composition and 
metabolism of the microbiota can synergize with their direct effects, or those of other bioactives, to enhance or alter their bioactivity. Importantly, for compounds with unknown mechanisms but observed phenotypic responses, it is entirely possible that the function is completely microbialdependent and the efficacy, therefore, contingent on the presence of the microbe(s) and their metabolic response to the dietary stimulus.

\section{Hypothesis and Specific Aims}

Our overall goal is to better understand the impact of dietary bioactives on the composition and metabolism of the colon microbiota and how this may alter colon health. Our overarching hypothesis is that chemoprotective diets containing bioactive compounds will protect the host by modulating the composition, metabolism, and spatial distribution of the colon microbiota thereby mitigating the response to insults such as obesity, carcinogens, or radiation exposure. These investigations will employ a variety of experimental paradigms: (1) using a chemical inducer of colorectal cancer in a rat model of carcinogenesis, (2) using whole body HZE radiation exposure of C57BL/6 mice, and (3) using overweight/obese human subjects. The knowledge generated from these studies is important for understanding the development of colon cancer with an emphasis on the host-microbe interaction. These findings will enhance the development of novel disease prevention strategies which are dietary in nature, thereby mitigating the financial and healthcare burden imposed by chronic, metabolic diseases such as obesity, diabetes, cardiovascular disease, and cancer.

Aim 1: Determine the ability of dried plums to suppress colon cancer by creating a microenvironment that promotes a healthy colonic epithelium and beneficial microbial growth and metabolism during AOM-induced colon carcinogenesis. 
Hypothesis 1.1: AOM-induced alterations in bacterial populations, fecal SCFA concentrations, and the expression of genes related to microbial recognition, inflammation, and SCFA absorption and metabolism will be mitigated by dried plum consumption

Hypothesis 1.2: Dried plum consumption, via alterations to the composition and metabolism of colonic bacteria, will increase plant- and microbe-derived metabolites throughout the colon.

Hypothesis 1.3: Consumption of dried plum will reduce the incidence of pre-neoplastic lesions induced by exposure to AOM.

Hypothesis 1.4: Consumption of dried plum will increase the number of apoptotic cells in the distal colon.

Aim 2: Determine if dried plum consumption can mitigate alterations in the gut microbiota and luminal SCFA levels in mice exposed to HZE radiation.

Hypothesis 2.1: HZE exposure will affect the colon luminal environment by modifying bacterial populations and luminal SCFA concentrations.

Hypothesis 2.2: Dried plum consumption will protect against alterations in bacterial composition and luminal SCFA levels that occur as a result of HZE radiation exposure.

Aim 3: Determine if a sumac-sorghum dietary intervention containing condensed tannins is capable of modifying fecal microbiota composition and the presence of metabolites of microbial origin in the feces and plasma of overweight human subjects to improve biomarkers related to metabolic syndrome.

Hypothesis 3.1: Consumption of a sumac-sorghum dietary intervention will beneficially impact fasting glucose, triglyceride and cholesterol levels, and plasma biomarkers of inflammation in human subjects. 
Hypothesis 3.2: Consumption of a sumac-sorghum dietary intervention rich in condensed tannins will increase the abundance of bacteria associated with improvements in conditions contributing to metabolic syndrome.

Hypothesis 3.2: Consumption of a sumac-sorghum dietary intervention will increase plasma and fecal concentrations of microbially-derived metabolites of sorghum/polyphenol origin in overweight human subjects and modify fecal short chain fatty acid concentrations. 


\section{CHAPTER II}

\section{DRIED PLUMS PROTECT AGAINST CHEMICALLY-INDUCED COLON CARCINOGENESIS IN SPRAGUE DAWLEY RATS}

\section{Introduction}

Despite a continued overall decline in incidence and mortality, colorectal cancer (CRC) remains the third most commonly diagnosed cancer among men and women in the United States (239). In contrast, the incidence of colorectal cancer for individuals under the age of 50 is increasing and is solely due to tumors of the distal colon and rectum (239). Recently, the American Cancer Society recommended the screening age for CRC be lowered to 45 years for average risk individuals. This recommendation is likely driven by $\mathrm{CRC}$ data indicating individuals aged $\leq 50$ years old with $\mathrm{CRC}$ often present with more advanced cancers and a concomitant higher risk of mortality (239). Therefore, colon cancer chemoprevention is necessary to reduce the contribution of colon cancer to the national healthcare burden. Interventions that are easily achievable in daily routines and incorporated into dietary patterns are more likely to be successful countermeasures.

Epidemiological studies have revealed that consumption of fruits, vegetables, and dietary fiber contributes to a reduction in CRC risk (33-35). The chemoprotective activity afforded by fruit and vegetable consumption is believed to be due to the fiber content, antioxidant compounds, and other bioactive compounds (i.e., extra-nutritional elements in food that can affect living tissue) $(33,34,36)$. Moreover, certain food constituents can be metabolized by gut microbiota to produce secondary bioactive compounds that then affect host physiology, such as the fermentation of dietary fiber into short chain fatty acids (SCFA) $(1,37)$. Differences in host 
and microbial physiology between the proximal and distal colon likely contribute to the unique pathology of cancers at these sites $(18,78,137)$. Therefore, dietary strategies which promote beneficial microbial populations and patterns of metabolism throughout the colon may better protect against CRC.

The digestive benefits of dried plums have been recognized for ages, yet a clear picture of the mechanisms whereby the digestive tract benefits remains to be developed. Dried plums contain a variety of bioactive compounds, such as phenolic acids (e.g., quercetin, chlorogenic acid, and neochlorogenic acid), sugar alcohols (e.g., sorbitol and mannitol), and hydroxycinnamic acids (e.g., sinapinic acid), as well as a mixture of soluble and insoluble fiber $(194,195)$. Dried plum consumption is commonly associated with a reduction in constipation and improved intestinal motility (194). These effects can be attributed in part to the dietary fiber found in dried plums; however, they are also noted when consuming dried plum juice, which lacks the fiber found in the whole fruit. Therefore, not all of the bioactive properties ascribed to dried plum consumption are due to its fiber content.

Development of an undesirable population of colonic bacteria promotes colon inflammation, a known promoter of colon cancer (3). One mediator of inflammation that is greatly elevated in colon cancer is COX-2 (240). We have previously demonstrated a reduction in activation of NF- $\mathrm{\kappa B}$ and $\mathrm{COX}-2$ expression in healthy rats consuming quercetin or chlorogenic acid (196). Quercetin and chlorogenic acid also influence fecal SCFA concentrations, suggesting alterations in the gut microbe composition, their metabolism, or in the absorption of SCFA by the host (196). Furthermore, our lab has demonstrated the ability of quercetin to reduce the numbers of HMACF. Part of these changes may be attributable to the maintenance of more normal levels of expression of TOLLIP, I $\mathrm{B} \alpha$, and butyrate transporters 
that were all reduced in animals challenged with an inflammatory agent (198). These outcomes indicate changes in microbial metabolism and in expression of genes involved in mediating signaling between bacteria and colonocytes in response to dietary exposure to polyphenolic molecules. These data from our lab were generated using isolated compounds, which precluded our understanding of potential additive or synergistic effects generated by a complete food, such as dried plum, which contain multiple bioactive compounds in complex with dietary fiber.

In this experiment, we compared microbial populations and their metabolic products in healthy rats and those exposed to a colon carcinogen while consuming either a basal diet or one containing dried plum. Profiling of the luminal microbiome and metabolome and epithelial gene expression analyses were conducted using samples from the proximal and distal colon segments to monitor the changes throughout the colon.

\section{Materials and Methods}

\section{$\underline{\text { Animals and experimental design }}$}

All animal work was approved by the Institutional Animal Care and Use Committee of Texas A\&M University (AUP number 2012-0252) and conformed to NIH guidelines. Fifty-one male Sprague Dawley rats (28-d old, Envigo, USA) were individually housed and acclimated to the specific pathogen-free animal facility for $1 \mathrm{wk}$ prior to starting the experimental diets (Figure 1). Rats were pre-fed their experimental diets for $3 \mathrm{wk}$ before receiving their first injection of azoxymethane (AOM, $15 \mathrm{mg} / \mathrm{kg}$ body weight) or saline (equivalent volume). Twenty rats served as saline controls and 31 rats received subcutaneous injections of azoxymethane to induce colon carcinogenesis. All rats were given a second injection 1 wk later and terminated 8 wk following the second injection by $\mathrm{CO}_{2}$ asphyxiation and cervical dislocation. Within the saline group, 10 rats received the basal diet $(\mathrm{BD}+\mathrm{S})$ and 10 rats received the dried plum diet 
$(\mathrm{PD}+\mathrm{S})$. Within the AOM group, 15 rats received the basal diet $(\mathrm{BD}+\mathrm{A})$ and 16 rats received the dried plum diet (PD+A). (One extra animal was received from Envigo and assigned to the PD+A group.) No animals died until their scheduled termination.

$\underline{\text { Experimental diets }}$

Proximate analysis of the dried plum puree (Sunsweet Growers, Inc., Yuba City, CA) was conducted (Covance Laboratories, Princeton, NJ) to determine macronutrient levels prior to diet formulation to maintain essentially equivalent macronutrient content and fiber solubility between the basal and plum diet. The dried plum puree was included at $5 \%$ of total calories, which is similar to the recommended daily serving size of dried plums (40 g) and its corresponding calorie content (100 calories) for a normal 2000 calorie diet. The powdered diets were a modified standard AIN-76A formulation (Appendix Table 1) and were provided ad libitum (198).

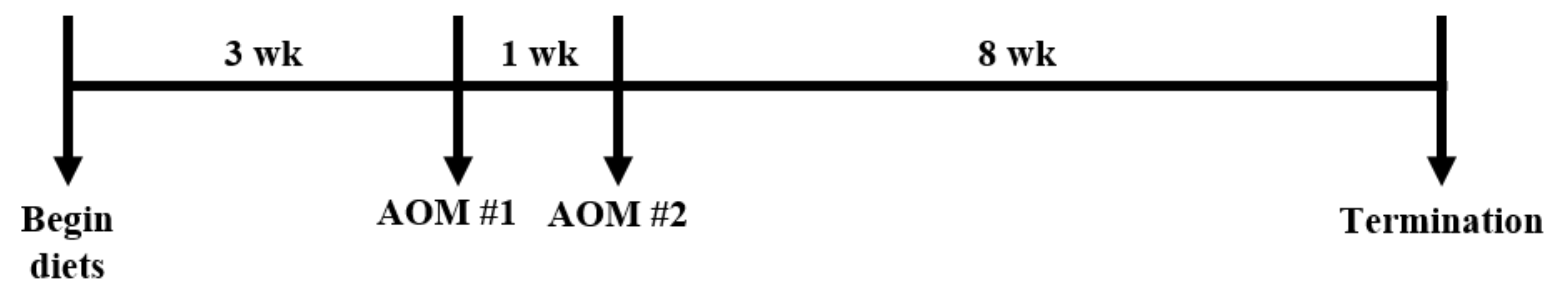

Figure 1. Timeline of AOM treatments for AOM group rats in each experimental group. Saline animals were injected with saline at the same time as their AOM counterparts. After 3 wk of experimental diets, AOM injections were given $(15 \mathrm{mg} / \mathrm{kg}$ body weight, 1 wk separation, 2 injections total).

\section{$\underline{\text { Sample collection }}$}

Animal weight and food intake was measured at several time points (167). Multiple fecal samples were collected during the experiment $(\mathrm{d} 21,28,84)$ and at termination when colon 
tissues were collected. Colons were excised and $1 \mathrm{~cm}$ of the proximal and distal colon (241) was fixed in $4 \%$ paraformaldehyde. Luminal contents collected from the proximal and distal segments were immediately flash frozen in liquid nitrogen. The colon was flushed with ice cold phosphate buffered saline ( $\mathrm{pH} 7.4)$ and opened longitudinally to expose the mucosal surface. One longitudinal half-section was fixed for aberrant crypt foci (ACF) analysis and the other halfsection was cut into proximal and distal segments for mucosal scraping.

\section{$\underline{\text { Aberrant crypt foci }}$}

The number and multiplicity of ACF was determined in ethanol-fixed tissue using our standard procedures $(180,181)$. Briefly, the tissues were fixed in $70 \%$ ethanol for $24 \mathrm{~h}$ and then placed in fresh $70 \%$ ethanol until scoring. The specimens were stained with $0.05 \%$ methylene blue dye and observed under a light microscope. The number, multiplicity, and location of each aberrant crypt or ACF was recorded in a blinded fashion and the identity of the sample remained unknown until all samples had been scored.

$\underline{\text { In situ apoptosis measurement }}$

Apoptosis was measured by TdT-mediated UTP-biotin nick end labeling (TUNEL) assay (ApopTag Plus Peroxidase In Situ Apoptosis Detection Kit; S7101; EMD Millipore) of fragmented pieces of DNA using $4 \mu \mathrm{m}$ sections of PFA-fixed, paraffin-embedded tissue as previously described (242). See Appendix B for full protocol. Apoptotic cells with condensed chromatin, apoptotic bodies and intense brown staining were counted in 30 crypt columns for each animal. Results are reported as the number of apoptotic cells per crypt column.

\section{Colonocyte gene expression}

Scraped mucosal samples from the proximal and distal colon were placed into RNA protection solution (Ambion, Grand Island, NY, USA) and stored at $-80^{\circ} \mathrm{C}$ in preparation for 
analysis using our standard procedures $(243,244)$. Real-time PCR was performed using Applied Biosystems (Foster City, CA) card arrays on a 7900HT Fast Real-Time PCR System for the following genes: Toll-like receptor 2 and 4 (Tlr2, Tlr4), Toll interacting protein (Tollip), Solute carrier family 16 member 1 (Slc16a1), Solute carrier family 5 member 8 (Slc5a8), Nuclear factor kappa b subunit 1 (Nfkbl), NFKB inhibitor alpha (Nfkbia), Prostaglandin-endoperoxide synthase 2 (Ptgs2), Nitric oxide synthase 2 (Nos2), Acyl-CoA dehydrogenase short chain (Acads), and Acyl-CoA dehydrogenase medium chain (Acadm)(245). The expression of Gapdh transcripts in each sample was used to normalize gene expression. Assay IDs for all primers are compiled in Appendix Table 2. Quality RNA could not be obtained for one BD+A rat ( $n=14$ of 15) and two PD+A rats ( $n=14$ of 16$)$.

\section{$\underline{\text { SCFA analysis }}$}

The SCFA analyses were conducted using our standard protocols $(153,167)$. Frozen fecal samples were ground, mixed with an internal standard (2-ethylbutyric acid), extracted in $70 \%$ ethanol, and aliquots of supernatant were mixed with heptanoic acid prior to injection onto a HP-FFAP column in a Varian 3900 GC (Agilent Technologies, Santa Clara, CA).

Concentrations in the samples were determined by comparison to a commercially available mix of standards.

\section{$\underline{\text { Microbial DNA isolation }}$}

Luminal contents collected from the proximal and distal colon at termination were flash frozen and stored at $-80^{\circ} \mathrm{C}$. Three samples were not available from the $\mathrm{BD}+\mathrm{A}$ rats, leaving 13 and 14 samples from the proximal and distal colon, respectively in that group. DNA was isolated using the FastDNA SPIN kit for feces (MP Biomedicals, Solon, OH) (103) and DNA 
concentration was determined using a Quant-iT ${ }^{\mathrm{TM}}$ PicoGreen ${ }^{\circledR}$ dsDNA Assay Kit (Invitrogen, Carlsbad, CA) in preparation for downstream 16S rRNA gene surveys (103). Microbial sequencing (454/Roche pyrosequencing)

Total microbial DNA was amplified using forward (27F: 5'AGAGTTTGATCCTGGCTCAG-3') and reverse (338R: 5'-TGCTGCCTCCCGTAGGAGT-3') primers targeting regions flanking the variable regions 1 through 3 (V1-3) of the bacterial 16S rRNA gene (246). The forward primer was tagged with $10 \mathrm{bp}$ unique barcode labels at the 5 ' end and with the adaptor sequence to allow multiple samples to be included in a single 454 GS FLX Titanium+ sequencing plate at a sequencing depth of approximately 10,000 reads per sample $(247,248)$. The sequencing was performed on a GS FLX Titanium+ instrument (Roche, Florence, SC) in the Microbiome Core Facility at University of North Carolina School of Medicine at Chapel Hill.

\section{$\underline{\text { Bacterial sequence analysis }}$}

Analysis of sequencing data was carried out using the QIIME pipeline (249) in which raw sequencing data and relevant metadata describing the samples were de-multiplexed and filtered. Next, data were denoised using Denoiser software with standard parameters (250) and sequences were grouped into operational taxonomic units (OTUs) at a 97\% level to approximate genuslevel phylotypes using Uclust (251). OTU sequences were aligned and OTU tables containing the counts of each OTU in each sample were used to calculate mean species diversity of each sample (alpha diversity) and the differentiation among samples (beta diversity). Alpha and beta diversity measures were used to calculate the Shannon-Weaver diversity index for each sample. 


\section{$\underline{\text { Microbial metagenome prediction }}$}

The Phylogenetic Investigation of Communities by Reconstruction of Unobserved States (PICRUSt) was used to predict the microbial metagenome (220). Briefly, sequencing data were prepared as described for taxonomic identification, but sequences were then clustered into OTUs using a closed-reference OTU picking protocol [http://qiime.org/scripts/pick_closed_reference_o tus.html] using Greengenes 13.8 as the reference database at $97 \%$ sequence similarity. The OTU table generated from this procedure was then normalized by the predicted copy number(s) of the microbial 16s rRNA gene for each OTU. PICRUSt analysis was then used with the normalized OTU table to predict each samples metagenome. The metagenome profile is calculated by multiplying the normalized OTU abundance by each KEGG ortholog (KO) abundance in the genome and summing the $\mathrm{KO}$ abundances together for each sample. This process yields a table containing $\mathrm{KO}$ abundances for each metagenome sample in the input OTU table. The data was then rarefied to an even sequencing depth (10000 sequences/sample) before further analyses were conducted.

\section{$\underline{\text { Metabolomics }}$}

Untargeted metabolic profiling of fecal samples was performed by Metabolon Inc. (Durham, NC) $(252,253)$. Briefly, the protocol employed for this analysis combined three independent platforms: ultrahigh performance liquid chromatography/tandem mass spectrometry (UHPLC/MS/MS ${ }^{2}$ ) optimized for basic species, UHPLC/MS/MS ${ }^{2}$ optimized for acidic species, and gas chromatography/mass spectrometry (GC/MS). Protein was precipitated from the fecal samples with methanol and the resulting supernatant was split into equal aliquots for analysis on the three platforms. Metabolites were identified by automated comparison of the ion features in the experimental samples to a reference library of chemical standard entries that included 
retention time, molecular weight $(\mathrm{m} / \mathrm{z})$, preferred adducts, and in-source fragments as well as associated MS spectra, and were curated by visual inspection for quality control using software developed at Metabolon (254).

$\underline{\text { Statistical analyses }}$

ACF data were analyzed by 2-sample t-test using GraphPad (PRISM) software. Apoptosis data was analyzed by one-way ANOVA. Microbial abundance and gene expression data were analyzed using the General Linear Model (GLM) procedure in SAS (version 9.3) to determine the main effects and interaction of diet and injection for the proximal and distal colon separately. Paired sample t-tests were used to compare proximal and distal expression levels within a group (e.g., $\mathrm{BD}+\mathrm{S}$ proximal versus $\mathrm{BD}+\mathrm{S}$ distal). Interactions between location and diet/injection were not tested. Permutational analysis of variance (PERMANOVA) was performed on unweighted beta-diversity principal coordinate data to determine if clustering due to effect variables was significant. SCFA data from each time point were analyzed separately. At baseline SCFA data were analyzed using a 2-sample t-test to determine differences in fecal SCFAs exclusively attributed to diet. SCFA data from $48 \mathrm{~h}$ and 8 wk after the second AOM injection were analyzed using the GLM procedure in SAS for diet, injection and diet*injection interaction. Data presented are least squares means $(\mathrm{LSM}) \pm$ standard error of the mean (SEM) unless otherwise noted. Differences are considered significant at $\mathrm{p}<0.05$.

PICRUSt data at Level 3 KEGG pathway consolidation were first filtered to remove KO functions with $>50 \%$ zeros across all samples. The resulting data was checked for normality and a square root transform or ranked test was used for non-normal KO functions. The KO functions were then analyzed by a 2-factor ANOVA (diet and injection) for the proximal and distal colon separately. The resulting raw $P$-values were corrected for false discovery rate (FDR) and the 
adjusted FDR $P$-value ( $Q$-value) is presented. A $Q$-value $<0.1$ was considered significant. The same was performed for Level 2 KEGG pathway analysis except the $>50 \%$ zeros exclusion was not necessary at this level.

For statistical analyses and data display purposes of the metabolomics data, any missing values were assumed to be below the limits of detection and these values were imputed with the compound minimum (minimum value imputation) (255). Statistical analysis of log-transformed data was performed using "R" (http://cran.r-project.org/). Pairwise comparisons were performed using Welsh's t-Tests. Multiple comparisons were accounted for by estimating the false discovery rate (FDR) using $Q$-values (256).

\section{Results}

Animal health

Food intake and animal weight were not adversely affected by the treatments (Figure 2 and 3). Food intake averaged over a $48 \mathrm{~h}$ period was elevated for PD rats at $3 \mathrm{wk}$ compared to BD rats (21.09 g vs. $18.89 \mathrm{~g} ; P<0.05)$; however, no differences in food intake were observed among the groups at later time points. At $3 \mathrm{wk}, \mathrm{PD}+\mathrm{S}$ rats weighed more than $\mathrm{BD}+\mathrm{A}$ rats $(255 \mathrm{~g}$ vs. $244 \mathrm{~g} ; P<0.05)$. At termination, rats that had received AOM injections weighed $4.32 \%$ less than their saline counterparts $(P<0.05)$.

Aberrant crypt foci and apoptosis

Neither injection nor diet had an impact on colon length (Table 1). As expected, there were no aberrant crypts in animals treated with saline. $\mathrm{BD}+\mathrm{A}$ animals had $73 \%$ more $(P<0.05)$ aberrant crypts (AC) and 63\% more $(P<0.05)$ ACF than PD+A animals (Table 2). Most importantly, the number of high multiplicity aberrant crypt foci (HMACF, foci containing four or more AC), which are believed to be most indicative of eventual tumor formation $(257,258)$, 


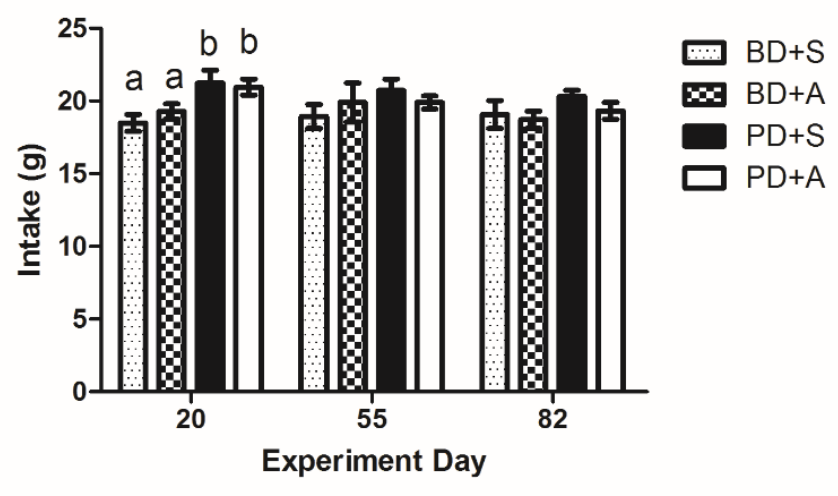

Figure 2. Daily food intake (averaged over $48 \mathrm{~h}$ ) at experimental day 20, 55, and 82. At d 20, animals assigned to the plum diet had increased diet intake, compared to basal diet counterparts. Data are means \pm SEM. At each time point, bars not sharing a common letter differ significantly $(P<0.05)$.

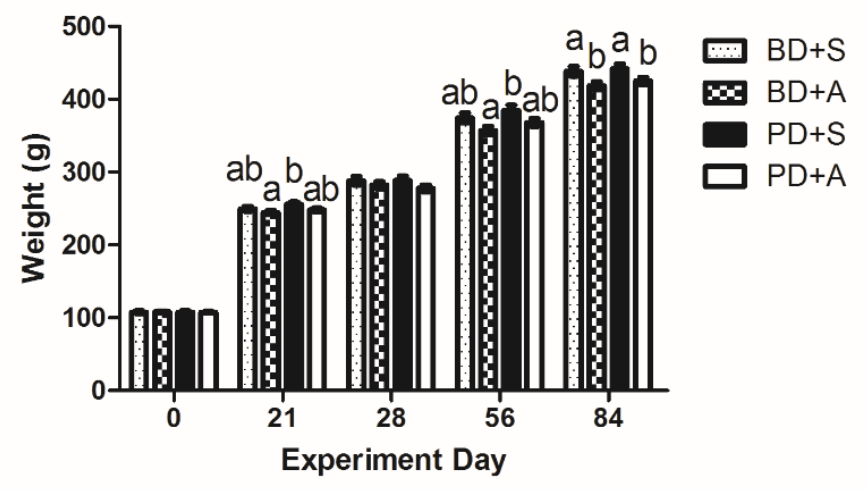

Figure 3. Average body weights at experimental day 0, 21, 28, 56 and 84. At d 21 and 56, PD+S animals weighed more than $\mathrm{BD}+\mathrm{A}$ animals. At $\mathrm{d} 84, \mathrm{PD}+\mathrm{S}$ animals weighed more than $\mathrm{BD}+\mathrm{A}$ and $\mathrm{PD}+\mathrm{A}$ animals, and $\mathrm{BD}+\mathrm{S}$ animals weighed more than $\mathrm{BD}+\mathrm{A}$ animals. Data are means \pm SEM. At each time point, bars not sharing a common letter differ significantly $(P<0.05)$.

Table 1. Total number of $\mathrm{AC}^{1}, \mathrm{ACF}$, and $\mathrm{HMACF}$ were reduced in rats treated with $\mathrm{AOM}$ and fed a dried plum diet, versus basal diet counterparts.

\begin{tabular}{|r|c|c|c|c|c|}
\hline Group $^{2}$ & n & Colon length $(\mathbf{c m})$ & Total AC & ACF & HMACF \\
\hline BD+S & 10 & $11.5 \pm 1.0$ & 0 & 0 & 0 \\
\hline PD+S & 10 & $11.0 \pm 1.0$ & 0 & 0 & 0 \\
\hline BD+A & 15 & $11.0 \pm 1.5$ & $156 \pm 20$ & $49 \pm 06$ & $18 \pm 02$ \\
\hline PD+A & 16 & $11.5 \pm 1.5$ & $90 \pm 11^{*}$ & $30 \pm 03^{*}$ & $9 \pm 02^{*}$ \\
\hline
\end{tabular}

${ }^{1} \mathrm{AC}$, aberrant crypts; ACF, aberrant crypt foci; HMACF, high multiplicity aberrant crypt foci (foci with number of $\mathrm{AC} \geq 4)$. ${ }^{2} \mathrm{BD}+\mathrm{S}$, basal diet+saline; $\mathrm{PD}+\mathrm{S}$, plum diet+saline; $\mathrm{BD}+\mathrm{A}$, basal diet+AOM; PD+A, plum diet+AOM. Data are means \pm SEM. $*$ indicates different from $\mathrm{BD}+\mathrm{A},(P<0.05)$. 
were $100 \%$ greater in the $\mathrm{BD}+\mathrm{A}$ rats compared to $\mathrm{PD}+\mathrm{A}$ rats $(P<0.05)$. No differences were detected in the number of apoptotic cells/crypt column or in the proportion of apoptotic cells/crypt column in the distal colon (Appendix Figure 1 and 2, respectively).

\section{$\underline{\text { Gene expression }}$}

To better understand how the diet may be affecting the cells of the colonic epithelium, genes involved in host-microbe crosstalk, as well as select genes down-stream of this interaction, were assessed for their relative expression levels (Table 2). The majority of genes surveyed were differentially expressed between the proximal and distal colon. $T l r 2$, which recognizes a variety of microbial cell-wall components, such as peptidoglycan matrix, lipoteichoic acid and various lipoproteins, was expressed at higher levels in the distal colon than the proximal colon in all rats, but was unaffected by diet or AOM treatment in the distal colon.

In the proximal colon, $\mathrm{PD}$ animals had lower $T l r 2$ expression than BD rats $(P$ diet $=0.0491$ ) with $\mathrm{PD}+\mathrm{A}$ animals being significantly lower than $\mathrm{BD}+\mathrm{S}$ and $\mathrm{BD}+\mathrm{A}$ groups $(P<0.05)$. Similarly, the expression of $T l r 4$, which recognizes lipopolysaccharide, was also greater in the distal colon of all animals $(\mathrm{P}<0.05)$. $\mathrm{PD}+\mathrm{A}$ rats had significantly lower Tlr4 expression than $\mathrm{BD}+\mathrm{A}$ rats $(P$-interaction $=0.0107)$.

Expression of Slc5a8 $\left(\mathrm{Na}^{+}\right.$coupled) and $\mathrm{Slcl} 6 a l\left(\mathrm{H}^{+}\right.$coupled) SCFA transporters was greater in the distal colon of all groups except for BD+A animals, whose proximal and distal Slc16a1 levels were equivalent. The expression of Slc $5 a 8$ was unaffected by diet or AOM injection regardless of location; however, Slc16al expression was reduced in the distal colon as a result of AOM treatment ( $P-\mathrm{AOM}=0.0013)$. The expression of $N f k b 1$ and its inhibitor, $N f k b i a$, was unaffected by diet or AOM treatment. Expression of Nos2 was greater in the distal colon 
Table 2. Colonocyte gene expression ${ }^{1}$ levels in rats treated with AOM, or saline, and fed a basal or dried plum diet.

\begin{tabular}{|c|c|c|c|c|c|c|c|}
\hline $\begin{array}{l}\text { Proximal } \\
\text { Colon }\end{array}$ & \multicolumn{4}{|c|}{ Gene expression normalized to Gapdh } & \multicolumn{2}{|c|}{$\begin{array}{c}\text { Main Effect } \\
P \text {-value }\end{array}$} & \multirow{2}{*}{$\begin{array}{c}\begin{array}{c}\text { Interaction } \\
P \text {-value }\end{array} \\
\text { Diet*AOM }\end{array}$} \\
\hline Gene & $\begin{array}{c}\mathbf{B D}+\mathbf{S} \\
\mathbf{n}=\mathbf{1 0}\end{array}$ & $\begin{array}{c}\mathrm{BD}+\mathrm{A} \\
\mathrm{n}=14\end{array}$ & $\begin{array}{c}\mathbf{P D}+\mathbf{S} \\
\mathbf{n}=10\end{array}$ & $\begin{array}{c}\mathrm{PD}+\mathrm{A} \\
\mathrm{n}=14\end{array}$ & Diet & AOM & \\
\hline$T l r 2$ & $9.96 \pm 1.38^{\mathrm{a}}$ & $10.37 \pm 1.17^{\mathrm{a}}$ & $8.89 \pm 1.38^{\mathrm{ab}}$ & $6.25 \pm 1.17^{\mathrm{b}}$ & 0.0491 & 0.3908 & 0.2411 \\
\hline Tlr4 & $3.03 \pm 0.47^{\mathrm{ab}}$ & $4.20 \pm 0.40^{\mathrm{a}}$ & $3.70 \pm 0.47^{\mathrm{ab}}$ & $2.55 \pm 0.40^{\mathrm{b}}$ & 0.2623 & 0.9836 & 0.0107 \\
\hline Tollip & $2.31 \pm 0.44$ & $2.67 \pm 0.37$ & $2.68 \pm 0.44$ & $1.94 \pm 0.37$ & 0.6583 & 0.6477 & 0.1815 \\
\hline Slc5a8 & $7.19 \pm 1.53$ & $8.61 \pm 1.30$ & $8.27 \pm 1.53$ & $6.05 \pm 1.30$ & 0.6036 & 0.7797 & 0.2068 \\
\hline Slc16al & $1.32 \pm 0.21$ & $1.50 \pm 0.18$ & $1.58 \pm 0.21$ & $1.20 \pm 0.18$ & 0.9206 & 0.6286 & 0.1621 \\
\hline$N f k b 1$ & $2.53 \pm 0.47$ & $3.05 \pm 0.39$ & $2.98 \pm 0.47$ & $2.26 \pm 0.39$ & 0.7040 & 0.8207 & 0.1558 \\
\hline Nfkbia & $8.44 \pm 1.38$ & $9.39 \pm 1.17$ & $9.20 \pm 1.38$ & $6.58 \pm 1.17$ & 0.4258 & 0.5161 & 0.1697 \\
\hline Ptgs2 (Cox2) & $10.64 \pm 1.14$ & $9.63 \pm 0.97$ & $10.64 \pm 1.14$ & $7.80 \pm 0.97$ & 0.3887 & 0.0755 & 0.3923 \\
\hline Nos2 (Inos) & $1.82 \pm 0.61^{\mathrm{ab}}$ & $3.36 \pm 0.52^{\mathrm{a}}$ & $0.81 \pm 0.65^{b}$ & $1.41 \pm 0.52^{\mathrm{b}}$ & 0.0139 & 0.0697 & 0.4236 \\
\hline Acads & $9.13 \pm 1.55$ & $10.71 \pm 1.31$ & $11.95 \pm 1.55$ & $9.02 \pm 1.31$ & 0.6960 & 0.6410 & 0.1244 \\
\hline Acadm & $8.41 \pm 1.25$ & $9.71 \pm 1.06$ & $9.79 \pm 1.25$ & $7.20 \pm 1.06$ & 0.6280 & 0.5833 & 0.1001 \\
\hline Distal Colon & \multicolumn{4}{|c|}{ Gene expression normalized to Gapdh } & \multicolumn{2}{|c|}{$\begin{array}{l}\text { Main Effect } \\
P \text {-value }\end{array}$} & $\begin{array}{l}\text { Interaction } \\
P \text {-value }\end{array}$ \\
\hline Gene & $\begin{array}{c}\mathbf{B D}+\mathbf{S} \\
\mathbf{n}=\mathbf{1 0}\end{array}$ & $\begin{array}{c}\mathrm{BD}+\mathrm{A} \\
\mathrm{n}=14\end{array}$ & $\begin{array}{c}\mathbf{P D}+\mathbf{S} \\
\mathbf{n}=\mathbf{1 0}\end{array}$ & $\begin{array}{c}\begin{array}{c}\text { PD+A } \\
\text { n=14 }\end{array} \\
\end{array}$ & Diet & AOM & Diet*AOM \\
\hline$T l r 2$ & $20.19 \pm 3.17$ & $20.87 \pm 2.68$ & $20.01 \pm 3.17$ & $21.22 \pm 2.68$ & 0.9768 & 0.7490 & 0.9284 \\
\hline Tlr4 & $12.75 \pm 1.21$ & $12.73 \pm 1.02$ & $11.68 \pm 1.21$ & $10.87 \pm 1.02$ & 0.1970 & 0.7124 & 0.7263 \\
\hline Tollip & $4.83 \pm 0.56$ & $3.80 \pm 0.48$ & $4.66 \pm 0.56$ & $3.88 \pm 0.48$ & 0.9319 & 0.0894 & 0.8040 \\
\hline Slc5a8 & $21.75 \pm 2.93$ & $16.14 \pm 2.48$ & $21.59 \pm 2.93$ & $18.36 \pm 2.48$ & 0.7068 & 0.1101 & 0.6637 \\
\hline Slc16al & $2.32 \pm 0.24^{\mathrm{ac}}$ & $1.57 \pm 0.21^{\mathrm{b}}$ & $2.55 \pm 0.24^{\mathrm{c}}$ & $1.74 \pm 0.21^{\mathrm{ab}}$ & 0.3966 & 0.0013 & 0.8954 \\
\hline Nfkbl & $4.95 \pm 0.69$ & $4.39 \pm 0.58$ & $5.00 \pm 0.69$ & $4.52 \pm 0.58$ & 0.8949 & 0.4149 & 0.9532 \\
\hline Nfkbia & $16.97 \pm 2.11$ & $14.13 \pm 1.79$ & $17.37 \pm 2.11$ & $14.06 \pm 1.79$ & 0.9340 & 0.1231 & 0.9061 \\
\hline $\operatorname{Ptgs} 2$ (Cox2) & $21.85 \pm 2.40$ & $17.75 \pm 2.03$ & $18.37 \pm 2.40$ & $15.66 \pm 2.03$ & 0.2170 & 0.1322 & 0.7560 \\
\hline Nos2 (Inos) & $1.42 \pm 1.05^{\mathrm{a}}$ & $8.17 \pm 0.89^{b}$ & $1.84 \pm 1.05^{\mathrm{a}}$ & $6.02 \pm 0.89^{\mathrm{b}}$ & 0.3753 & $<0.0001$ & 0.1919 \\
\hline Acads & $19.64 \pm 2.30$ & $14.57 \pm 1.94$ & $20.39 \pm 2.30$ & $16.54 \pm 1.94$ & 0.5247 & 0.0417 & 0.7752 \\
\hline \multirow[t]{2}{*}{ Acadm } & $13.40 \pm 1.38^{\mathrm{ab}}$ & $9.95 \pm 1.16^{\mathrm{a}}$ & $13.87 \pm 1.38^{\mathrm{b}}$ & $10.84 \pm 1.16^{\mathrm{ab}}$ & 0.5990 & 0.0146 & 0.8694 \\
\hline & \multicolumn{4}{|c|}{ Fold change (Distal/Proximal) } & & & \\
\hline Gene & BD+S & BD+A & PD+S & $\mathbf{P D}+\mathbf{A}$ & & & \\
\hline Tlr2 & $2.03 *$ & $2.01 *$ & $2.25 *$ & $3.40 *$ & & & \\
\hline Tlr4 & $4.21 *$ & $3.03 *$ & $3.16^{*}$ & $4.26 *$ & & & \\
\hline Tollip & $2.09 *$ & $1.42 *$ & $1.74 *$ & $2.00 *$ & & & \\
\hline Slc5a8 & $3.03 *$ & $1.88 *$ & $2.61 *$ & $3.04 *$ & & & \\
\hline Slcl6al & $1.76^{*}$ & 1.05 & $1.61 *$ & $1.45^{*}$ & & & \\
\hline$N f k b 1$ & $1.96 *$ & 1.44 & $1.68 *$ & $2.00 *$ & & & \\
\hline Nfkbia & $2.01 *$ & $1.51 *$ & $1.89 *$ & $2.14 *$ & & & \\
\hline Ptgs2 (Cox2) & $2.05 *$ & $1.84 *$ & $1.73 *$ & $2.01 *$ & & & \\
\hline Nos2 (Inos) & $0.78 *$ & $2.43 *$ & $2.27 *$ & $4.27 *$ & & & \\
\hline Acads & $2.15 *$ & 1.36 & $1.71 *$ & $1.83 *$ & & & \\
\hline Acadm & $1.59 *$ & 1.03 & 1.42 & $1.51 *$ & & & \\
\hline
\end{tabular}

${ }^{1}$ Relative amount of target gene normalized to Gapdh. BD+S, basal diet+saline; BD+A, basal diet+AOM; PD+S, plum diet+saline; PD+A, plum diet+AOM. Data are least square means \pm SEM. ${ }^{\text {a,b,c }}$ Values in the same row not sharing a common superscript differ, $(P<0.05)$. * Indicates significant difference in gene expression between proximal and distal colon for that gene within the group $(P<0.05)$. 
for all groups except for $\mathrm{BD}+\mathrm{S}$, which had greater expression in the proximal colon. The expression of Nos 2 was increased in response to AOM treatment, but only in the distal colon $(P$ $\mathrm{AOM}<0.0001)$. In the proximal colon, PD rats had lower Nos 2 expression $(P$-diet $=0.0139)$ than $\mathrm{BD}$ rats, with $\mathrm{PD}+\mathrm{A}$ rats having significantly lower expression than $\mathrm{BD}+\mathrm{A}$ rats.

\section{Colon microbiota}

Fecal microbial characterization was carried out using samples collected from the proximal and distal colon at termination. Diet and AOM treatment affected the abundance of several bacterial phyla throughout the colon (Table 3). AOM treatment increased the abundance of Bacteroidetes in the distal colon $(P-\mathrm{AOM}=0.0285)$ irrespective of diet. Changes in various genera in response to AOM are presented in Appendix Table 1. The impact of PD consumption on microbe composition appeared to be more pronounced in the distal colon, where changes in four phyla were observed versus only two in the proximal colon. In the proximal colon, PD rats had higher abundances of Actinobacteria $(P$-diet $=0.0003)$ and Proteobacteria $(P$-diet $=0.0158)$ than $\mathrm{BD}$ rats. In the distal colon, PD rats, had higher abundances of Actinobacteria $(P-$ diet $=0.0003)$ and Bacteroidetes $(P$-diet $=0.0003)$, and lower abundances of Deferribacteres $(P$ diet $=0.0119)$, Firmicutes $(P$-diet $=0.0054)$, and bacteria classified as 'Other' $(P$-diet $=0.0102)$ than $\mathrm{BD}$ rats.

At the order level, the abundance of Bacteroidales was significantly higher in the distal colon of PD rats versus BD rats $(P$-diet $<0.001)$ (Figure 4). Lactobacillales was higher in BD+S rats than all other groups in the proximal and distal colon $(P<0.05)$, and PD animals had significantly lower abundances in the proximal and distal colon compared to BD $(P$-diet $=0.0248$ and 0.0011, for each location respectively) (Figure 4). The abundance of Clostridiales was higher 
Table 3. Percent abundance of dominant phyla in rats treated with AOM, or saline, and fed a basal or dried plum diet.

\begin{tabular}{|c|c|c|c|c|c|c|c|}
\hline Proximal Colon & \multicolumn{4}{|c|}{ Microbial abundance, $\%$ of sequences } & \multicolumn{2}{|c|}{$\begin{array}{c}\text { Main Effect } \\
P \text {-value }\end{array}$} & \multirow{2}{*}{$\begin{array}{c}\begin{array}{c}\text { Interaction } \\
P \text {-value }\end{array} \\
\text { Diet*AOM }\end{array}$} \\
\hline Phylum & $\begin{array}{c}\text { BD+S } \\
n=10 \\
\end{array}$ & $\begin{array}{c}\text { BD+A } \\
n=13 \\
\end{array}$ & $\begin{array}{c}\text { PD+S } \\
n=10\end{array}$ & $\begin{array}{c}\text { PD+A } \\
n=16\end{array}$ & Diet & AOM & \\
\hline Unassigned & $4.88 \pm 2.79$ & $6.35 \pm 2.44$ & $7.73 \pm 2.79$ & $5.24 \pm 2.20$ & 0.7357 & 0.8444 & 0.4450 \\
\hline Other & $2.42 \pm 0.45$ & $1.89 \pm 0.40$ & $1.70 \pm 0.45$ & $2.30 \pm 0.36$ & 0.7173 & 0.9350 & 0.1859 \\
\hline Actinobacteria & $0.12 \pm 0.07^{\mathrm{a}}$ & $0.24 \pm 0.06^{\mathrm{ac}}$ & $0.54 \pm 0.07^{\mathrm{b}}$ & $0.33 \pm 0.06^{\mathrm{c}}$ & 0.0003 & 0.5146 & 0.0176 \\
\hline Bacteroidetes & $29.24 \pm 3.24^{\mathrm{ab}}$ & $21.29 \pm 2.84^{\mathrm{a}}$ & $31.54 \pm 3.24^{\mathrm{b}}$ & $28.04 \pm 2.56^{\mathrm{ab}}$ & 0.1361 & 0.0616 & 0.4596 \\
\hline Cyanobacteria & $0.11 \pm 0.09^{\mathrm{a}}$ & $0.14 \pm 0.08^{\mathrm{a}}$ & $0.17 \pm 0.09^{\mathrm{ab}}$ & $0.38 \pm 0.07^{\mathrm{b}}$ & 0.0573 & 0.1247 & 0.2777 \\
\hline Deferribacteres & $0.03 \pm 0.01$ & $0.02 \pm 0.01$ & n.d. & $0.02 \pm 0.01$ & 0.1527 & 0.8430 & 0.1052 \\
\hline Elusimicrobia & $0.04 \pm 0.02^{\mathrm{ab}}$ & $0.04 \pm 0.02^{\mathrm{ab}}$ & $0.02 \pm 0.02^{\mathrm{a}}$ & $0.07 \pm 0.01^{\mathrm{b}}$ & 0.7109 & 0.1209 & 0.1770 \\
\hline Firmicutes & $60.00 \pm 4.48^{\mathrm{ab}}$ & $65.62 \pm 3.93^{\mathrm{a}}$ & $53.28 \pm 4.47^{\mathrm{b}}$ & $57.90 \pm 3.54^{\mathrm{ab}}$ & 0.0872 & 0.2205 & 0.9109 \\
\hline Fusobacteria & $<0.01$ & $<0.01$ & $<0.01$ & $<0.01$ & 0.4992 & 0.6672 & 0.9448 \\
\hline Proteobacteria & $2.85 \pm 0.68^{a}$ & $3.60 \pm 0.60^{\mathrm{ab}}$ & $4.59 \pm 0.68^{\mathrm{ab}}$ & $5.03 \pm 0.54^{\mathrm{b}}$ & 0.0158 & 0.3507 & 0.8022 \\
\hline Tenericutes & $0.31 \pm 0.30$ & $0.76 \pm 0.26$ & $0.35 \pm 0.30$ & $0.69 \pm 0.24$ & 0.9609 & 0.1563 & 0.8417 \\
\hline Verrucomicrobia & $0.03 \pm 0.03$ & $0.06 \pm 0.03$ & $0.08 \pm 0.04$ & $<0.01$ & 0.9573 & 0.4277 & 0.1734 \\
\hline Distal Colon & \multicolumn{4}{|c|}{ Microbial abundance, $\%$ of sequences } & \multicolumn{2}{|c|}{$\begin{array}{c}\text { Main Effect } \\
P \text {-value }\end{array}$} & $\begin{array}{c}\text { Interaction } \\
P \text {-value }\end{array}$ \\
\hline Phylum & $\begin{array}{c}\text { BD+S } \\
n=10\end{array}$ & $\begin{array}{c}\text { BD+A } \\
n=14\end{array}$ & $\begin{array}{c}\text { PD+S } \\
n=10\end{array}$ & $\begin{array}{c}P D+A \\
n=16\end{array}$ & Diet & AOM & Diet*AOM \\
\hline Unassigned & $5.60 \pm 1.24$ & $2.80 \pm 1.04$ & $3.33 \pm 1.24$ & $2.76 \pm 0.98$ & 0.3116 & 0.1423 & 0.3308 \\
\hline Other & $2.71 \pm 0.55^{\mathrm{a}}$ & $1.79 \pm 0.46^{\mathrm{ab}}$ & $1.00 \pm 0.55^{\mathrm{b}}$ & $0.81 \pm 0.43^{\mathrm{b}}$ & 0.0102 & 0.2753 & 0.4717 \\
\hline Actinobacteria & $0.18 \pm 0.09^{\mathrm{a}}$ & $0.31 \pm 0.08^{\mathrm{a}}$ & $0.54 \pm 0.09^{\mathrm{b}}$ & $0.59 \pm 0.07^{\mathrm{b}}$ & 0.0003 & 0.2891 & 0.6193 \\
\hline Bacteroidetes & $23.99 \pm 3.00^{\mathrm{a}}$ & $27.50 \pm 2.54^{\mathrm{a}}$ & $32.07 \pm 3.00^{\mathrm{a}}$ & $40.96 \pm 2.37^{\mathrm{b}}$ & 0.0003 & 0.0285 & 0.3315 \\
\hline Cyanobacteria & $0.17 \pm 0.07$ & $0.15 \pm 0.06$ & $0.24 \pm 0.07$ & $0.18 \pm 0.05$ & 0.4270 & 0.5380 & 0.7275 \\
\hline Deferribacteres & $0.08 \pm 0.02^{\mathrm{a}}$ & $0.02 \pm 0.02^{\mathrm{b}}$ & $<0.01^{\mathrm{b}}$ & n.d. & 0.0119 & 0.1009 & 0.1032 \\
\hline Elusimicrobia & $0.05 \pm 0.02$ & $0.04 \pm 0.02$ & $0.06 \pm 0.02$ & $0.08 \pm 0.02$ & 0.3093 & 0.8913 & 0.4648 \\
\hline Firmicutes & $62.98 \pm 3.34^{\mathrm{a}}$ & $63.01 \pm 2.82^{\mathrm{a}}$ & $58.89 \pm 3.34^{\mathrm{a}}$ & $49.26 \pm 2.64^{\mathrm{b}}$ & 0.0054 & 0.1227 & 0.1203 \\
\hline Fusobacteria & $<0.01$ & $<0.01$ & n.d. & $<0.01$ & 0.1822 & 0.0834 & 0.6966 \\
\hline Proteobacteria & $3.42 \pm 0.66$ & $3.60 \pm 0.56$ & $3.34 \pm 0.66$ & $4.39 \pm 0.52$ & 0.5576 & 0.3171 & 0.4773 \\
\hline Tenericutes & $0.76 \pm 0.25$ & $0.72 \pm 0.21$ & $0.52 \pm 0.25$ & $0.96 \pm 0.20$ & 0.9968 & 0.3827 & 0.3080 \\
\hline Verrucomicrobia & $0.07 \pm 0.04$ & $0.06 \pm 0.03$ & $0.01 \pm 0.04$ & $0.01 \pm 0.03$ & 0.1039 & 0.8781 & 0.7367 \\
\hline
\end{tabular}

${ }^{1} \mathrm{BD}+\mathrm{S}$, basal diet+saline; BD+A, basal diet+AOM; PD+S, plum diet+saline; PD+A, plum diet+AOM. Data are least square means \pm SEM. a,b Values in the same row not sharing a common superscript differ, $(P<0.05)$. "n.d." denotes sequences were not detected in any samples of the respective group. Phyla representing less than $0.01 \%$ of sequences are noted as such. 

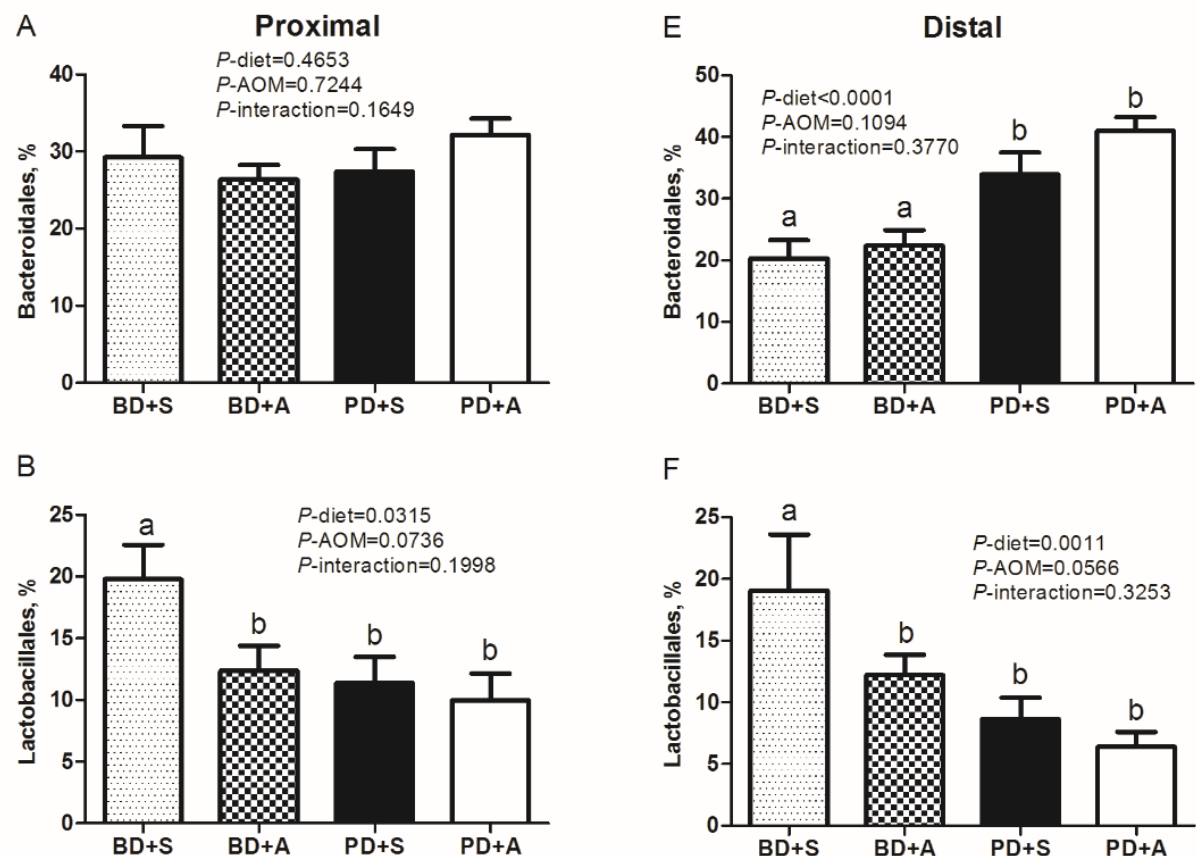

$\mathrm{F}$

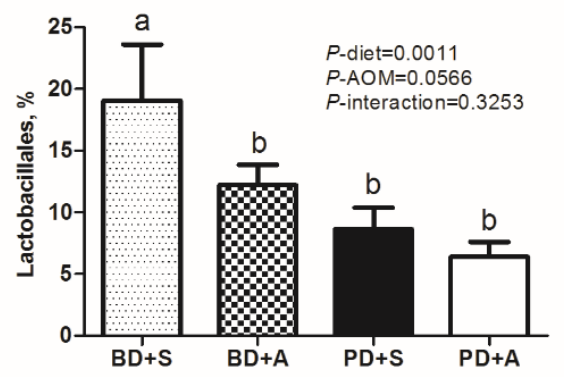

C

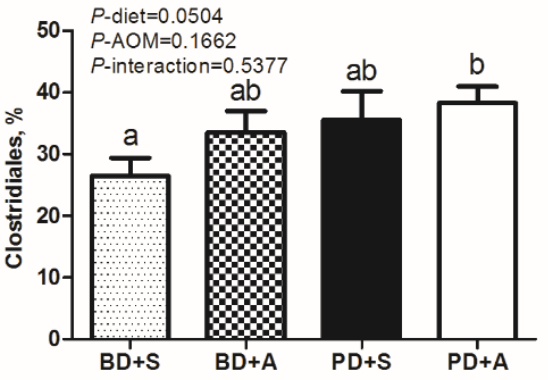

G

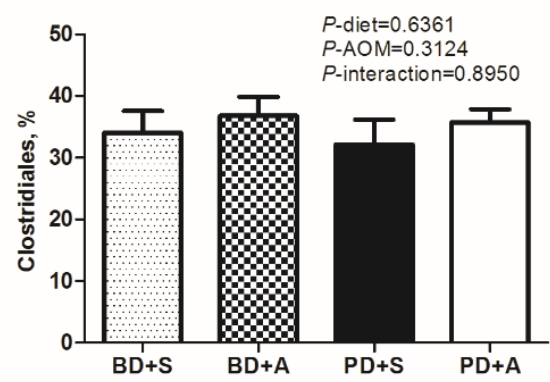

D

$\mathrm{H}$
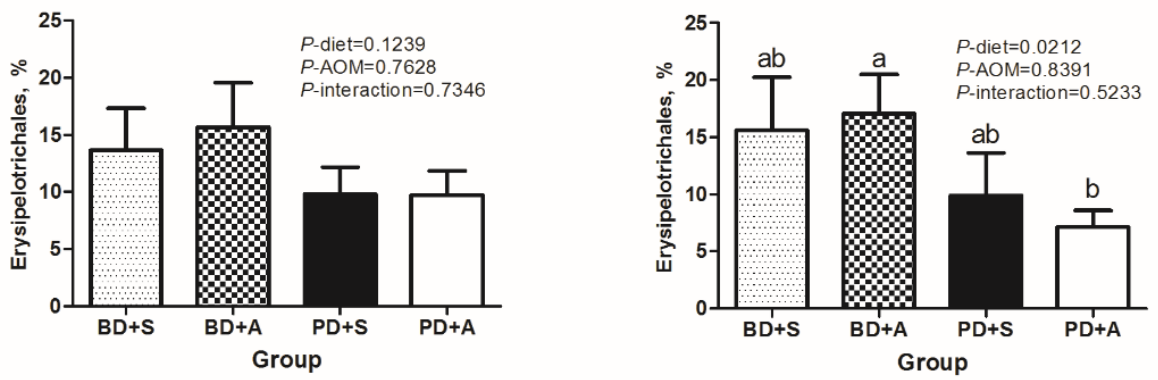

Figure 4. Percent abundance of major bacterial orders by diet/injection group and location. Bars without a common letter are significantly different $(P<0.05)$. Data are means \pm SEM. 
in the proximal colon of $\mathrm{PD}+\mathrm{A}$ rats compared to $\mathrm{BD}+\mathrm{S}$ rats $(P<0.05)$, but no differences were detected in the distal colon. PD rats had lower abundances of Erysipelotrichales than BD rats in the distal colon $(P$-diet $=0.0212)$. Additionally, Coriobacteriales, which largely comprises the Actinobacteria phylum, was higher in PD rats, irrespective of location surveyed compared to BD rats $(P$-diet $<0.001$ for both locations, data not shown). A number of significant alterations occurred in various genera throughout the colon with potential health relevance in response to AOM treatment and diet consumed (Appendix Table 3 and 4, respectively). For example, PD rats had increased abundances of Bacteroides ( 64\%, P-diet=0.0005), Parabacteroides ( 132\%, $P$-diet $=0.0024), S 247(\sim 34 \%, P$-diet=0.0268), and decreased Lactobacillus $(\sim 48 \%, P$ diet=0.0011) and Allobaculum $(\sim 52 \%, P$-diet=0.021) (Appendix Table 2).

Alpha-diversity estimates representing diversity within a sample (e.g., Chao, Shannon, and total number of observed species) revealed no significant differences between samples due to diet, AOM treatment, or location surveyed (data not shown). Beta-diversity analysis, or diversity between samples, illustrated with principal coordinate analysis (Figure 5) and confirmed by PERMANOVA of principal coordinate data (Table 4), revealed significant separation between samples taken from different diet groups without species abundance weighting factors ( $P$-diet=0.001). Principal coordinates analysis of weighted beta-diversity analysis, which considers species abundance, is presented in Figure 6.

Microbial metagenome prediction

Thousands of predicted KEGG ortholog functions can be organized into KEGG Pathways using the categorize_by_function.py script. KEGG pathway data can take on three levels (i.e., Level 1->Level 2->Level 3) similar to microbial taxon organization (e.g., Phylum->Class- 


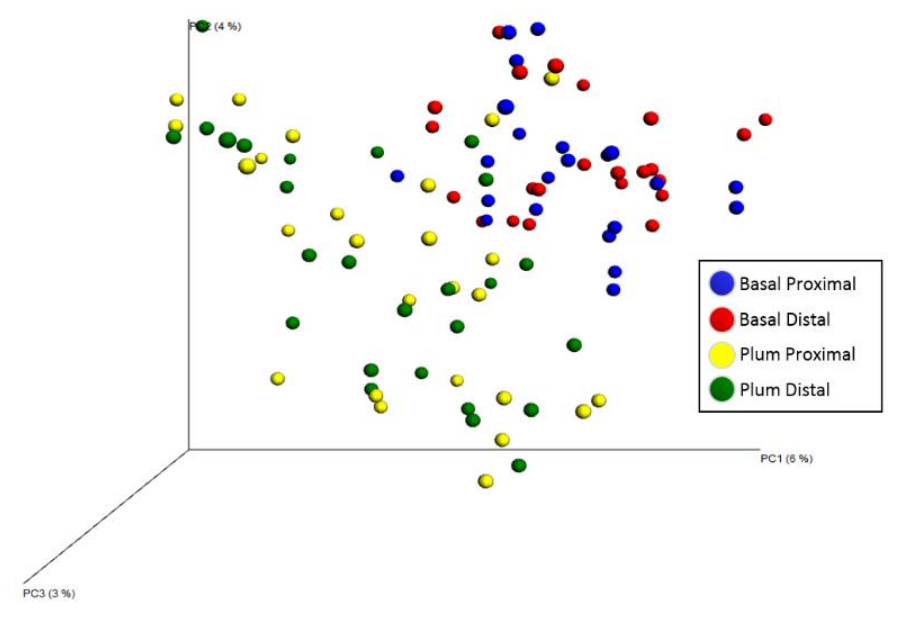

Figure 5. Unweighted UniFrac beta-diversity analysis illustrated with principle coordinate plots reveals separation of samples based on diet.

Table 4. Results of PERMANOVA analysis on unweighted beta-diversity principal coordinates data.

\begin{tabular}{|l|l|l|c|c|c|}
\hline Variable & Sample size & \# groups & F-statistic & $\boldsymbol{P}$-value & \# permutations \\
\hline Diet & 99 & 2 (Plum vs. Basal) & 4.101 & 0.001 & 999 \\
\hline Injection & 99 & 2 (AOM vs. Saline) & 1.211 & 0.060 & 999 \\
\hline Location & 99 & 2 (Proximal vs. Distal) & 0.957 & 0.581 & 999 \\
\hline
\end{tabular}

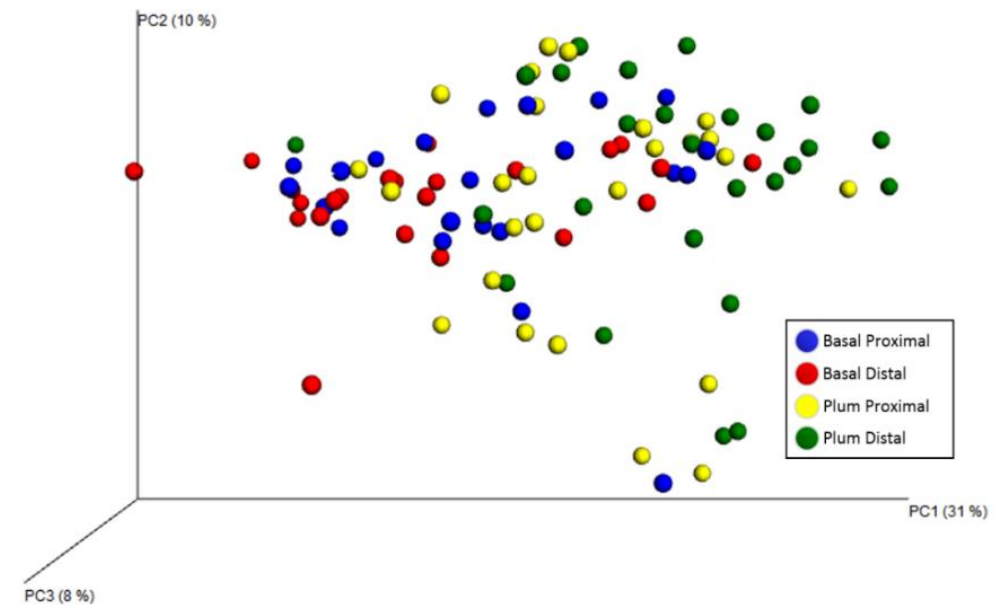

Figure 6. Weighted UniFrac beta-diversity analysis illustrated with principle coordinate plots reveals separation of samples based on diet with maximum separation occurring between distal colon microbial samples. 
>Order). Level 1 data is the broadest functional characterization with the following categories: Cellular Processes, Environmental Information and Processing, Genetic Information Processing, Human Diseases, Metabolism, Organismal Systems, and Unclassified. No changes were detected in Level 2 or 3 pathway data in response to AOM treatment for either colon location. Analysis of Level 2 data revealed six pathways altered by diet in the proximal colon and 27 pathways altered by diet in the distal colon (Table 5). For example, in the proximal colon KEGG pathways associated with Energy Metabolism, Amino Acid Metabolism, and Metabolism of Cofactors and Vitamins were significantly altered in response to diet. In the distal colon, KEGG pathways related to Membrane Transport, Cancers, Infectious Diseases, Energy Metabolism, Metabolism of Other Secondary Metabolites, and Lipid Metabolism were among the 27 pathways found to be affected by diet. KEGG pathway consolidation at Level 3 provides the most information regarding functional changes in the predicted metagenome. Significant changes of select KEGG pathways at Level 3 consolidation are presented in Table 6. Interestingly, KEGG pathways related to Fatty acid biosynthesis, Indole alkaloid biosynthesis, and Phenylalanine, tyrosine, and tryptophan biosynthesis were modified by diet in the proximal and distal colon. Furthermore, KEGG pathways identified as colorectal cancer, pathways in cancer, tryptophan metabolism, primary and secondary bile acid biosynthesis, metabolism of xenobiotics by cytochrome P450, and polycyclic aromatic hydrocarbon degradation were identified as pathways modified by diet in the distal colon. Relative prevalence of gene families related to the KEGG pathways identified in Plum versus Basal animals is also indicated in Table 6 in the Comparison column. A post hoc Tukey's multiple comparison test of the Shannon-Weaver alpha diversity index for predicted KEGG functions revealed significant differences between PD and BD groups in the distal colon, 
Table 5. Predicted microbial metagenome KEGG functional pathways (Level 2 pathway consolidation) significantly altered by diet in the proximal and distal colon of rats consuming a basal or dried plum diet and treated with AOM or saline.

\begin{tabular}{|c|c|c|c|c|}
\hline \multicolumn{5}{|c|}{ Proximal Colon } \\
\hline Function Level 1 & Function Level 2 & $P$-value & $Q$-value & Transform \\
\hline Genetic Information Processing & Folding Sorting and Degradation & 0.000 & 0.008 & Rank \\
\hline Metabolism & Energy Metabolism & 0.000 & 0.000 & None \\
\hline Metabolism & Amino Acid Metabolism & 0.002 & 0.021 & None \\
\hline Metabolism & Metabolism of Cofactors and Vitamins & 0.005 & 0.030 & None \\
\hline Organismal Systems & Endocrine System & 0.004 & 0.030 & None \\
\hline Unclassified & Cellular Processes and Signaling & 0.002 & 0.020 & None \\
\hline \multicolumn{5}{|c|}{$\begin{array}{c}\text { Distal Colon } \\
\end{array}$} \\
\hline $\begin{array}{r}\text { Function Level } 1 \\
\end{array}$ & $\begin{array}{r}\text { Function Level } 2 \\
\end{array}$ & $P$-value & $Q$-value & Transform \\
\hline Cellular Processes & Transport and Catabolism & 0.000 & 0.000 & None \\
\hline Cellular Processes & Cell Motility & 0.000 & 0.001 & Rank \\
\hline Environmental Information Processing & Membrane Transport & 0.000 & 0.000 & Rank \\
\hline Genetic Information Processing & Folding Sorting and Degradation & 0.000 & 0.000 & Rank \\
\hline Genetic Information Processing & Transcription & 0.004 & 0.009 & SqRt \\
\hline Genetic Information Processing & Translation & 0.015 & 0.024 & Rank \\
\hline Human Diseases & Metabolic Diseases & 0.004 & 0.009 & None \\
\hline Human Diseases & Cardiovascular Diseases & 0.007 & 0.014 & Rank \\
\hline Human Diseases & Infectious Diseases & 0.009 & 0.015 & None \\
\hline Human Diseases & Cancers & 0.029 & 0.042 & SqRt \\
\hline Metabolism & Energy Metabolism & 0.000 & 0.000 & None \\
\hline Metabolism & Metabolism of Cofactors and Vitamins & 0.000 & 0.000 & None \\
\hline Metabolism & Glycan Biosynthesis and Metabolism & 0.000 & 0.000 & SqRt \\
\hline Metabolism & Amino Acid Metabolism & 0.000 & 0.000 & None \\
\hline Metabolism & Metabolism of Other Amino Acids & 0.001 & 0.002 & Rank \\
\hline Metabolism & Biosynthesis of Other Secondary Metabolites & 0.003 & 0.007 & None \\
\hline Metabolism & Lipid Metabolism & 0.004 & 0.009 & None \\
\hline Metabolism & Metabolism of Terpenoids and Polyketides & 0.008 & 0.014 & Rank \\
\hline Metabolism & Enzyme Families & 0.016 & 0.025 & Rank \\
\hline Organismal Systems & Endocrine System & 0.000 & 0.000 & None \\
\hline Metabolism & Biosynthesis of Other Secondary Metabolites & 0.003 & 0.007 & None \\
\hline Organismal Systems & Digestive System & 0.000 & 0.000 & Rank \\
\hline Organismal Systems & Environmental Adaptation & 0.000 & 0.000 & SqRt \\
\hline Organismal Systems & Immune System & 0.000 & 0.001 & None \\
\hline Organismal Systems & Excretory System & 0.002 & 0.004 & SqRt \\
\hline Organismal Systems & Circulatory System & 0.011 & 0.018 & Rank \\
\hline Organismal Systems & Nervous System & 0.028 & 0.042 & Rank \\
\hline Unclassified & Cellular Processes and Signaling & 0.000 & 0.000 & None \\
\hline
\end{tabular}


with PD groups having greater diversity than their BD counterparts (PD+A vs BD+A and PD+S vs $\mathrm{BD}+\mathrm{S}, P<0.05$ for both, data not shown). This increase in pathway diversity was not observed in the proximal colon. No differences between diet+injection groups were observed for the other alpha diversity indices (i.e., Chao and number of observed OTUs) in either colon location.

Table 6. Select predicted microbial metagenome KEGG functional pathways (Level 3 pathway consolidation) significantly altered by diet in the proximal and distal colon of rats consuming a basal or dried plum diet and treated with AOM or saline.

\begin{tabular}{|c|c|c|c|c|}
\hline \multicolumn{5}{|c|}{ Proximal Colon } \\
\hline $\begin{array}{r}\text { Function Level } 3 \\
\end{array}$ & $P$-value & $Q$-value & Transform & Comparison $^{1}$ \\
\hline Phe, Tyr and Trp biosynthesis & 0.001 & 0.022 & None & $\mathrm{P}>\mathrm{B}$ \\
\hline Indole alkaloid biosynthesis & 0.010 & 0.076 & Rank & $\mathrm{P}>\mathrm{B}$ \\
\hline Fatty acid biosynthesis & 0.006 & 0.060 & SqRt & $\mathrm{P}>\mathrm{B}$ \\
\hline \multicolumn{5}{|c|}{ Distal Colon } \\
\hline $\begin{array}{rr}\text { Function Level } 3 \\
\end{array}$ & $P$-value & $Q$-value & Transform & Comparison \\
\hline Colorectal cancer & 0.008 & 0.015 & Rank & $\mathrm{P}>\mathrm{B}$ \\
\hline Pathways in cancer & 0.027 & 0.048 & SqRt & $\mathrm{P}<\mathrm{B}$ \\
\hline Indole alkaloid biosynthesis & 0.000 & 0.000 & SqRt & $\mathrm{P}>\mathrm{B}$ \\
\hline Phe, Tyr and Trp biosynthesis & 0.000 & 0.000 & None & $\mathrm{P}>\mathrm{B}$ \\
\hline Tryptophan metabolism & 0.000 & 0.000 & None & $\mathrm{P}<\mathrm{B}$ \\
\hline Fatty acid biosynthesis & 0.000 & 0.000 & Rank & $\mathrm{P}>\mathrm{B}$ \\
\hline Primary bile acid biosynthesis & 0.027 & 0.048 & Rank & $\mathrm{P}>\mathrm{B}$ \\
\hline Secondary bile acid biosynthesis & 0.025 & 0.044 & Rank & $\mathrm{P}>\mathrm{B}$ \\
\hline Drug metabolism-cytochrome P450 & 0.006 & 0.012 & SqRt & $\mathrm{P}>\mathrm{B}$ \\
\hline Metabolism of xenobiotics by cytochrome P450 & 0.006 & 0.012 & Rank & $\mathrm{P}>\mathrm{B}$ \\
\hline Polycyclic aromatic hydrocarbon degradation & 0.037 & 0.061 & SqRt & $\mathrm{P}<\mathrm{B}$ \\
\hline Phe, Tyr and Trp biosynthesis & 0.001 & 0.022 & None & $\mathrm{P}>\mathrm{B}$ \\
\hline Indole alkaloid biosynthesis & 0.010 & 0.076 & Rank & $\mathrm{P}<\mathrm{B}$ \\
\hline Fatty acid biosynthesis & 0.006 & 0.060 & SqRt & $\mathrm{P}>\mathrm{B}$ \\
\hline
\end{tabular}

${ }^{1}$ Indicates which diet the prevalence of the gene families associated with the KEGG pathway was greater. Plum, P; Basal, B.

$\underline{\text { Fecal short chain fatty acids }}$

Short chain fatty acids are the metabolic end product of microbial fermentation of dietary fiber. Because both diets were matched for the level and composition of dietary fiber, the intent 
of this comparison was to understand how other components of dried plums may be affecting the metabolism of SCFAs.

Following 3 wk of experimental diet consumption, there was no difference in total fecal SCFA concentrations between the BD and PD rats ( $P$-diet=0.1048); however, PD rats had lower levels of isobutyrate $(P$-diet $<0.0001)$, isovalerate $(P$-diet $<0.0001)$, and valerate $(P$-diet $=0.0002)$

\section{(Table 7).}

Forty-eight hours after the second injection $\mathrm{BD}+\mathrm{A}$ rats had greater total SCFAs compared to $\mathrm{BD}+\mathrm{S}$ rats $(P$-interaction $=0.0469)$. The increase in total SCFA levels observed in $\mathrm{BD}+\mathrm{A}$ animals at this time point was largely due to an increase $(\sim 64 \%)$ in acetate. The level of propionate was greater in $\mathrm{BD}+\mathrm{A}$ vs. $\mathrm{BD}+\mathrm{S}$ rats; however, the levels of propionate were not affected by AOM in the PD rats $(P$-interaction=0.0094). The level of isobutyrate decreased following AOM injection $(P-\mathrm{AOM}=0.0049)$ and was lower overall in $\mathrm{PD}$ rats versus $\mathrm{BD}$ rats $(P$-diet<0.0001). Isovalerate and valerate also decreased following AOM injection $(P$ $\mathrm{AOM}=0.0114$ and 0.0445 , respectively) and were lower overall in PD rats versus BD rats ( $P$ diet $=0.0013$ and 0.0025 , respectively).

Eight weeks after the second injection, only propionate was significantly affected by diet with $\mathrm{PD}$ rats having greater levels than $\mathrm{BD}$ rats $(P$-diet=0.0229). Interestingly, the levels of fecal butyrate remained comparable between all groups throughout the study; however, the proportion of butyrate was greater in animals consuming the plum diet at the 3 wk post-diet start $(P$ diet $=0.0476)$ and $48 \mathrm{~h}$ post-AOM $2(P$-diet $=0.014)$ time points. 
Table 7. Rat fecal SCFA levels at 3 wk post-diet start, and $48 \mathrm{~h}$ and $8 \mathrm{wk}$ following the second AOM injection, organized by diet+injection group for each time point.

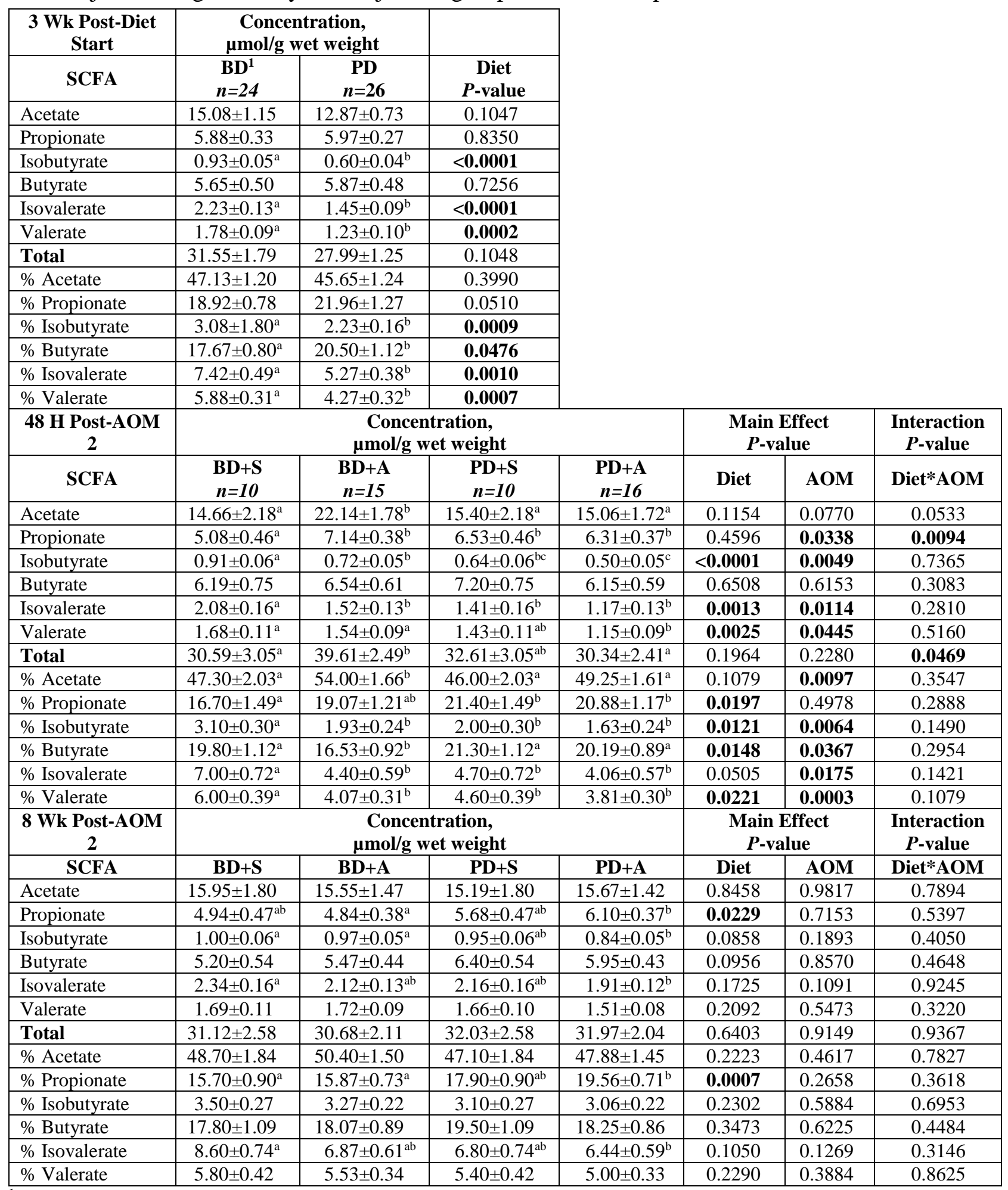

${ }^{1} \mathrm{BD}$, basal diet; $\mathrm{PD}$, plum diet; $\mathrm{BD}+\mathrm{S}$, basal diet+saline; $\mathrm{BD}+\mathrm{A}$, basal diet+AOM; PD+S, plum diet+saline; PD+A, plum diet+AOM. Data are means \pm SEM. ${ }^{\text {a,b }}$ Values not sharing a common super script differ, $(P<0.05)$. 


\section{Luminal metabolome profiling}

Based on our microbial data, dried plum consumption appears to exert its greatest influence on microbe composition in the distal colon. Similarly, the impact of AOM treatment on the expression of genes related to microbe recognition, SCFA metabolism, and inflammation was greatest in the distal colon. Thus, we sought to characterize the changes in the luminal metabolome in the distal colon to better understand the impact of dried plum consumption on colon health. The untargeted analyses of distal samples from BD+A and PD+A rats identified 490 metabolites. Between these groups, 147 metabolites were different $(P$-diet $\leq 0.05$, data not shown) and of these, 104 where upregulated in PD+A animals. The top 30 metabolites significantly altered by dried plum consumption are presented in Table 8. As expected, PD+A rats had increased relative concentrations of a number of food-derived metabolites versus BD+A, such as the sugar alcohol compounds mannitol/sorbitol, which is one of the more concentrated compounds found in dried plums and often cited for its laxative effect (194, 203, 259). PD+A animals also had significant increases in 5-hydroxymethylfurfural (HMF), a compound derived from the dehydration of sugars and commonly formed when sugar-containing foods are exposed to heat (e.g., drying or cooking) versus BD+A (194). Dried plums are known to contain up to $2200 \mathrm{mg} / \mathrm{kg}$ of HMF and its primary metabolite, 5-hydroxymethyl-2-fuoric acid (HMFA), was also highly elevated in PD+A animals compared to BD+A (260). Similarly, a number of plantderived/associated compounds were elevated in $\mathrm{PD}+\mathrm{A}$ animals compared to $\mathrm{BD}+\mathrm{A}$, including: quinate, salicylate, gentisate, sinapate, $1 \mathrm{H}$-quinoline-2-one, and the amino acid asparagine, which is highly concentrated in dried plums (261). Furthermore, a number of microbially-derived metabolites were significantly altered in PD+A animals as a result of increased diversity in substrate afforded by dried plum in the diet. For example, the phytoestrogen 
Table 8. Top metabolites affected by dried plum in the distal colon, their super pathway classification, database identifiers (if known), and fold of change (PD+A/BD+A, Least square means). Metabolites are sorted by increasing $Q$-values (i.e., FDR adjusted $P$-value).

\begin{tabular}{|c|c|c|c|c|c|c|}
\hline Metabolite & Super Pathway & KEGG & HMDB & PUBMED & Fold of Change & $Q$-value \\
\hline 5-hydroxymethylfurfural & Xenobiotics & $\mathrm{C} 11101$ & HMDB34355 & 237332 & 637.65 & $0.00 E+00$ \\
\hline Quinate & Xenobiotics & $\mathrm{C} 00296$ & HMDB03072 & 6508 & 124.27 & $0.00 \mathrm{E}+00$ \\
\hline 4-hydroxycyclohexylcarboxylic acid & Xenobiotics & $\mathrm{C} 04404$ & HMDB01988 & 151138 & 39.62 & $0.00 \mathrm{E}+00$ \\
\hline $\mathrm{N}$-acetylvaline & Amino Acid & & HMDB11757 & 66789 & 27.57 & $0.00 \mathrm{E}+00$ \\
\hline Mannitol/sorbitol & Carbohydrate & $\mathrm{C} 00794$ & HMDB00247 & 5780 & 20.88 & $0.00 \mathrm{E}+00$ \\
\hline Gentisate & Amino Acid & $\mathrm{C} 00628$ & HMDB00152 & 3469 & 14.87 & $0.00 \mathrm{E}+00$ \\
\hline Sinapate & Xenobiotics & $\mathrm{C} 00482$ & HMDB32616 & 637775 & 6.22 & $0.00 \mathrm{E}+00$ \\
\hline Arabonate/xylonate & Carbohydrate & & & & 4.39 & $0.00 E+00$ \\
\hline 2-oxindole-3-acetate & Xenobiotics & & HMDB35514 & 3080590 & 7.59 & 7.00E-15 \\
\hline Enterolactone & Xenobiotics & & & 10685477 & 6.54 & 5.97E-13 \\
\hline Asparagine & Amino Acid & $\mathrm{C} 00152$ & HMDB00168 & 6267 & 4.10 & 9.28E-12 \\
\hline Carboxyethyl-GABA & Amino Acid & & HMDB02201 & 2572 & 2.87 & 1.86E-10 \\
\hline 1H-quinolin-2-one & Xenobiotics & C06415 & & 6038 & 7.13 & 6.49E-10 \\
\hline Homocitrulline & Amino Acid & $\mathrm{C} 02427$ & HMDB00679 & 65072 & 4.59 & 1.70E-09 \\
\hline Deoxycarnitine & Lipid & C01181 & HMDB01161 & 134 & 11.92 & $9.68 \mathrm{E}-09$ \\
\hline Maleate & Lipid & $\mathrm{C} 01384$ & HMDB00176 & 444266 & 2.82 & 2.25E-07 \\
\hline Enterodiol & Xenobiotics & & & 123725 & 6.00 & $2.28 \mathrm{E}-06$ \\
\hline N-methylpipecolate & Xenobiotics & & & 11862129 & 4.38 & $3.36 \mathrm{E}-06$ \\
\hline 2'-deoxyinosine & Nucleotide & $\mathrm{C} 05512$ & HMDB00071 & 65058 & 0.35 & 4.79E-06 \\
\hline 7-methylguanine & Nucleotide & $\mathrm{C} 02242$ & HMDB00897 & 11361 & 12.78 & $5.40 \mathrm{E}-06$ \\
\hline Bilirubin $(\mathrm{Z}, \mathrm{Z})$ & Cofactors/Vitamins & $\mathrm{C} 00486$ & HMDB00054 & 5280352 & 5.09 & 8.19E-06 \\
\hline Galactitol (dulcitol) & Cofactors/Vitamins & $\mathrm{C} 00486$ & HMDB00054 & 5280352 & 3.33 & 8.83E-06 \\
\hline 5-hydroxymethyl-2-furoic acid & Amino Acid & C20448 & HMDB02432 & 80642 & 5.54 & 1.51E-05 \\
\hline 2-oxo-1-pyrrolidinepropionate & Xenobiotics & & & 3146688 & 2.94 & 1.89E-05 \\
\hline N1-methyladenosine & Nucleotide & $\mathrm{C} 02494$ & HMDB03331 & 27476 & 1.96 & 2.21E-05 \\
\hline 1-methylimidazoleacetate & Amino Acid & $\mathrm{C} 05828$ & HMDB02820 & 75810 & 1.68 & 3.58E-05 \\
\hline N6-acetyllysine & Amino Acid & $\mathrm{C} 02727$ & HMDB00206 & 92832 & 2.21 & $4.34 \mathrm{E}-05$ \\
\hline Pipecolate & Amino Acid & $\mathrm{C} 00408$ & HMDB00070 & 849 & 3.39 & 6.02E-05 \\
\hline Hippurate & Xenobiotics & $\mathrm{C} 01586$ & HMDB00714 & 464 & 4.54 & $6.24 \mathrm{E}-05$ \\
\hline 1-methylxanthine & Xenobiotics & C16358 & HMDB10738 & 80220 & 12.33 & $8.96 \mathrm{E}-05$ \\
\hline Eicosapentaenoate (EPA; 20:5n3) & Lipid & C06428 & HMDB01999 & 446284 & 2.07 & 0.0832 \\
\hline Linoleate $(18: 2 \mathrm{n} 6)$ & Lipid & $\mathrm{C} 01595$ & HMDB00673 & 5280450 & 1.77 & 0.0831 \\
\hline Linolenate $[\alpha$ or $\gamma ;(18: 3 n 3$ or 6$)]$ & Lipid & $\mathrm{C} 06426$ & HMDB03073 & 5280934 & 1.86 & 0.0715 \\
\hline Dihomo-linolenate $(20: 3 n 3$ or $n 6)$ & Lipid & $\mathrm{C} 03242$ & HMDB02925 & 5280581 & 3.27 & 0.0161 \\
\hline Docosapentaenoate (n3 DPA; 22:5n3) & Lipid & C16513 & HMDB06528 & 6441454 & 2.27 & 0.1308 \\
\hline
\end{tabular}


compounds enterolactone and enterodiol, derived from microbial metabolism of plant lignans, were both significantly enriched in PD+A animals versus BD+A (262). Other microbial metabolites associated with plant intake were similarly increased, including: 4hydroxycyclohexylcarboxylic acid, hippurate, and 2-oxindole-3-acetate. Lastly, several polyunsaturated fatty acids were also found at greater levels in the distal colon of plum rats compared to basal counterparts, including: eicosapentaenoate (EPA; 20:5n3; 2.07-fold), linoleate (18:2n6; 1.77-fold), linolenate ( $\alpha$ or $\gamma 18: 3 \mathrm{n} 3$ or $6 ; 1.86$-fold), and dihomo-linolenate (20:3n3 or n6; 3.27-fold). Moreover, dihomo-linolenate was also significantly greater in the proximal colon of plum rats versus basal. Following these observations, a fatty acid profile of both diets was generated via gas chromatography (263) using two samples obtained from each diet. Diets were checked for palmitic acid (16:0), stearic acid (18:0), oleic acid (18:1N-9), linoleic acid (18:2N6), and alpha linoleic acid (18:3N-3). The results from this analysis are presented in Table 9 and provides some evidence that the diets themselves did not differ in their fatty acid profiles.

Table 9. Fatty acid profiles* of basal and plum diets used in the experiment.

\begin{tabular}{|l|l|l|l|l|l|}
\cline { 2 - 6 } \multicolumn{1}{c|}{} & \multicolumn{5}{c|}{ Average (\% mol) } \\
\hline Diet & Palmitic 16:0 & Stearic 18:0 & Oleic 18:1N-9 & Linoleic 18:2N-6 & Alpha-linoleic 18:3N-3 \\
\hline Basal $(\mathrm{n}=2)$ & 13.70 & 1.99 & 28.42 & 54.91 & 0.98 \\
\hline Plum $(\mathrm{n}=2)$ & 13.73 & 1.94 & 28.45 & 54.82 & 1.05 \\
\hline
\end{tabular}

*only mass $>0.05 \%$ from GC result were reported.

Ingenuity pathway analysis

To better interpret the changes observed in the metabolome data, Ingenuity Pathway Analysis (IPA; Qiagen) was employed to determine metabolic pathways that may be affected by dried plum consumption. IPA is a causal analysis platform for 'omics' data that allows the 
identification of new targets and/or candidate biomarkers within the context of biological systems. Its analyses are based on a 'master' network derived from the Ingenuity Knowledge Database, which is itself derived from experimental data (264). Due to the pronounced changes in the distal gut microbiota, fold change data of metabolites with known database identifiers were used to identify pathways impacted by the dried plum diet in the distal colon. The workflow utilized for the IPA analysis is presented in Figure 7.

The resulting analysis detected several canonical pathways altered by dried plum consumption. The top five most significant canonical pathways discovered in response to dried plum consumption included IL-10 signaling, L-cysteine degradation I, II, and III, and heme degradation. The canonical pathways, number of compounds related to the pathway found in the dataset, and $P$-value of the pathway relationship is seen in Table 10. IL-10 signaling and Heme degradation shared the same two metabolites, bilirubin and biliverdine, which were $\sim 5$-fold and $\sim 3$-fold higher, respectively, in the distal colon of plum diet animals. Similarly, all three

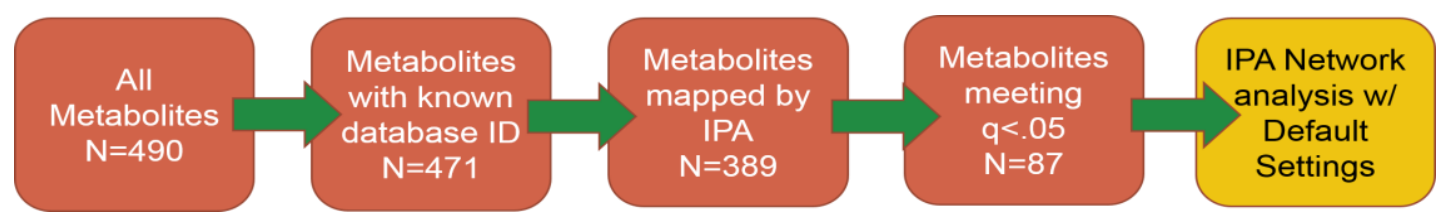

Figure 7. IPA workflow for network discovery using metabolites found to be significantly different between distal colon samples of Plum+AOM and Basal+AOM animals.

Table 10. Top canonical pathways detected by IPA software, number of compounds in the dataset related to the pathway, and their respective $P$-values.

\begin{tabular}{|l|l|l|}
\hline Top Canonical Pathways & $\#$ molecules & $P$-value \\
\hline IL-10 signaling & 2 & $5.12 \mathrm{E}-03$ \\
\hline L-cysteine degradation II & 2 & $5.12 \mathrm{E}-03$ \\
\hline Heme degradation & 2 & $1.69 \mathrm{E}-02$ \\
\hline L-cysteine degradation III & 2 & $1.69 \mathrm{E}-02$ \\
\hline L-cysteine degradation I & 2 & $1.69 \mathrm{E}-02$ \\
\hline
\end{tabular}


L-cysteine pathways shared the same compounds, L-cysteine and pyruvic acid, which were $~ 1.4-$ fold and 2.1-fold higher, respectively, in plum diet animals.

Use of IPA's Network discovery feature also revealed a number of potential relationships for investigation in future experiments aimed at further elucidating the bioactivity of dried plum in the colon. The top network associated with the distal colon metabolomics data in our AOM treated animals was comprised of three interrelated cellular functions: 1) Free radical scavenging, 2) Cell death and survival, and 3) Reproductive system development and function. The network generated from our input data and the Ingenuity Knowledge Database is presented in Figure 8.

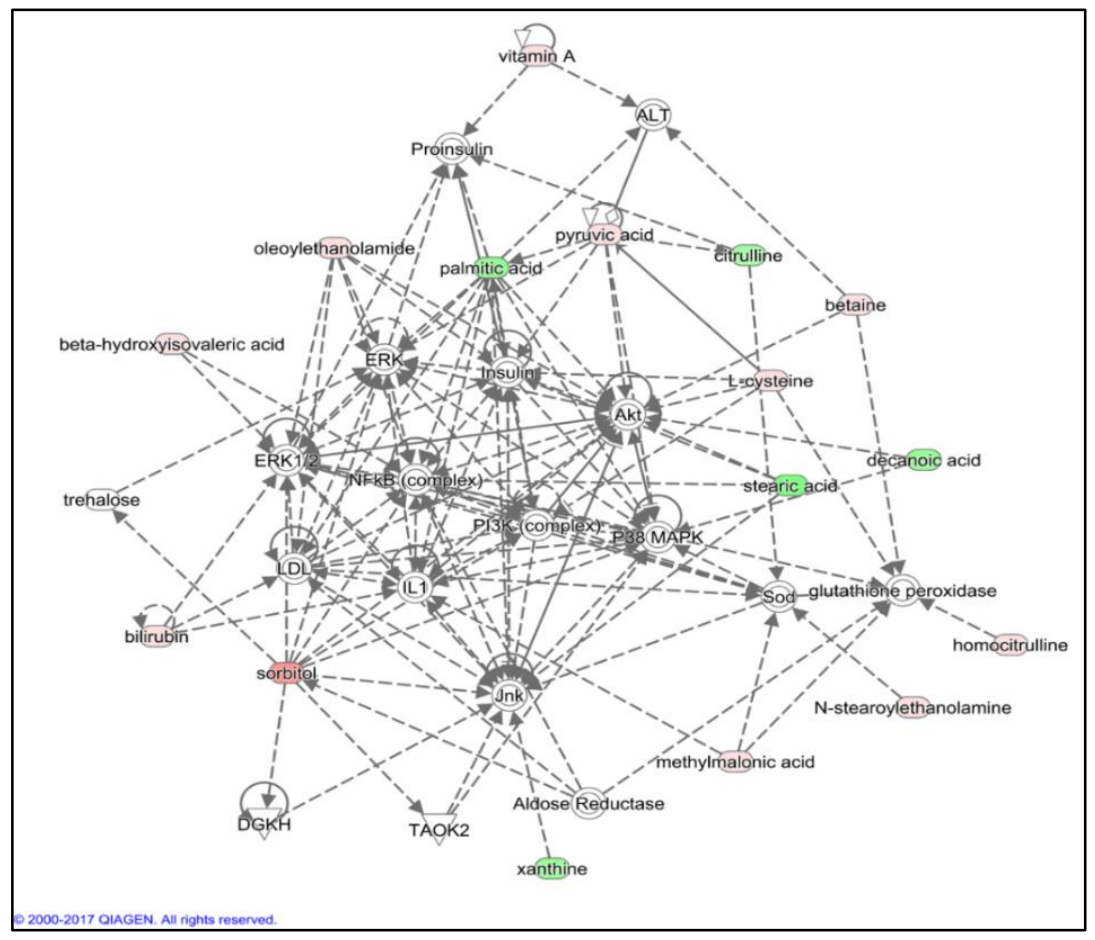

Figure 8. Graphical representation of the top ID Associated Network Functions generated by IPA: Free Radical Scavenging, Cell Death and Survival, Reproductive System Development and Function. Arrows indicate associations between nodes. Red and green indicate increases and decreases in metabolites, respectively. 


\section{Discussion}

The microbiota and their metabolites are known modifiers of cancer and inflammation, a well-established promoter of carcinogenesis (265). Therefore, understanding the contribution of the gut microbiota to colon cancer risk and progression and how the diet might impact this dynamic is a critical objective for disease prevention and treatment. The increased expression of inflammatory genes as a result of gene mutations, such as Nos 2 and Ptgs 2 contribute to the promotion phase of AOM-induced carcinogenesis in the model (266). Previous work using individual bioactive compounds derived from dried plums suggests chemoprotective and antiinflammatory activities that may be enhanced when consuming the whole fruit $(183,187,205$, $261,267,268)$.

A previous study by Yang and Gallaher (205) utilized a dried plum powder to investigate the impact of dried plum consumption on AOM-induced colon cancer. Despite no mitigation of pre-neoplastic lesion formation in response to AOM, they were able to document putatively beneficial microbial changes, specifically the abundance of genes involved in the generation of secondary bile acids, which are known mutagens (206). The objective of this study was to expand upon the current knowledge regarding the protective bioactivity of dried plums, and their impact on colon microbe composition and metabolism in the context of colon cancer. Incorporation of a dried plum puree, as $5 \%$ of total diet calories, reduced the incidence of HMACF induced by AOM treatment by 50\%. The increased expression of the inflammatory promoter Nos2 in the proximal colon following AOM treatment was mitigated in PD+A animals, whose levels remained comparable to BD+S animals. Tlr2 and Tlr4 are upstream of critical inflammatory pathways (e.g., $\mathrm{Nf} \kappa \beta$ ) which are activated by recognition of microbial pathogen associated molecular patterns (PAMPs) (269). The decrease in Tlr2 in PD+A animals versus 
$\mathrm{BD}+\mathrm{A}$, in combination with the reductions in $i N O S$, suggest that dried plum may be able to suppress the pro-inflammatory response to various microbial antigens that may otherwise exacerbate the response to AOM treatment. In the distal colon, neither diet was able to mitigate the AOM-induced changes in gene expression. Decreases in the expression of the proton coupled SCFA transporter, Slc16al, and the short- and medium chain acyl-CoA dehydrogenases in the distal colon of AOM-treated animals suggests an impaired ability to actively transport and metabolize SCFAs produced by gut bacteria. Decreased expression of Slc16al and similar transporters is known to occur during CRC (270). Higher relative expression of toll-like receptor, SCFA transporters and metabolism enzymes, and inflammatory genes in the distal colon suggests greater signaling activity between epithelial cells and the microbiota. Indeed, it is well established that the relative microbial load is greatest in the distal intestines (84).

This experiment demonstrated that the consumption of dried plum can modify microbial abundances throughout the colon, most notably, by decreasing the ratio of Firmicutes/Bacteroidetes in the distal colon. In the distal colon, Lactobacillus and Allobaculum contributed $\sim 30 \%$ of the bacterial sequences detected in basal diet animals, but only $\sim 15 \%$ in plum diet animals, suggesting substantial alterations in dominant bacteria when animals consume dried plum. Lactobacillus and Allobaculum are both gram-positive genera, which are specifically recognized by the Tlr2 receptor, whose gene expression was decreased in plum diet animals. Allobaculum-like bacteria have previously been associated with 1,2-dimethylhydrazine induction of colon cancer and the order they belong to (i.e., Erysipelotrichales) has been positively correlated with colon inflammation and CRC $(271,272)$. The alpha diversity indices of Chao, number of observed species, and Shannon index revealed no significant differences in the diversity of the samples because of diet, injection received, or location surveyed (data not 
shown). However, analysis of unweighted beta diversity revealed significant clustering of samples based on diet consumed. Altogether, these observations confirm that dried plum significantly impacts the composition of the colon microbiota including dramatic shifts in dominant bacterial groups; however, it is inappropriate to draw any conclusions regarding the mechanism of protection of dried plum consumption on the formation of ACF solely based on the observed changes in microbe composition. As previously mentioned, there is research associating certain groups of bacteria with specific disease states, but only a few have been mechanistically linked to contributing to disease conditions, such as hydrogen sulfide producing bacteria with colitis and colon cancer (273).

It has been argued that the functional profile of the microbiome is more important than the composition of the microbiota (215). Certainly, the information collected from the metagenome, and metatranscriptome, of the gut microbiota would help to better elucidate potential pathways governing the mechanisms associated with host-microbe interactions during healthy and diseased states. To gain a better understanding of the impact that the dried plum had on shaping the microbiota, OTU data were additionally analyzed using PICRUSt to predict the metagenome content of samples. Analysis of the PICRUSt data revealed significant alterations in several KEGG functional pathways. Because the OTU input file that is fed into PICRUSt is also used for the taxonomic characterization, we were not necessarily surprised that only the factor of diet had an impact on the predicted metagenome profiles. Furthermore, comparison of the number of significant changes in the proximal versus the distal colon agrees with the observation that diet had the greatest impact on microbe composition in the distal colon. PICRUSt analysis revealed several pathways altered by dried plum including several changes to pathways associated with the metabolism of fatty acids, amino acids, bile acids, and xenobiotics (e.g., 
polyphenol and plant constituents). Interestingly, plum diet animals were found to have a relatively greater prevalence of KEGG orthologs (KOs) related to secondary bile acid synthesis than basal diet animals, which is in contrast to work done by Yang and Gallaher (205). Because PICRUSt data relies on OTU data generated by sequencing a small portion of a single microbial gene (i.e., 16S rRNA), only the portion of the full metagenome contributed by the organisms targeted by the primers are included in the analysis. Furthermore, the use of a closed reference OTU reference (e.g., Green Genes), ancestral state reconstruction and inference of unknown microbe functions and $16 \mathrm{~S}$ copy number, inherently compounds the inaccuracy and, thus, absolute confidence in the data. Nevertheless, PICRUSt is an extremely useful tool for low cost prediction of changes in the metagenome and has been shown to produce $80-85 \%$ accurate metagenomes from $16 \mathrm{~S}$ data sets; however, it must be clearly stated that PICRUSt results are only suggestive and is most useful on human gut samples $(220,274)$.

Our assessment of the luminal metabolome in the distal colon allowed us to explore potential changes in microbial metabolism. Indeed, consumption of the dietary bioactives contained in DP may offer chemoprotective activities in their unmetabolized form. For example, sorbitol has been shown to induce apoptosis in human colorectal cancer cells by p38 MAPK signal transduction (204). Additionally, 5-HMF has demonstrated antioxidant and antiproliferative properties in vitro (275). A byproduct of heating and drying, 5-HMF levels may not be comparable in the undried fruit, but is known to exist at high concentrations in dried plum juice (276). Importantly, several microbial metabolites were affected by dried plum consumption. Enterodiol and enterolactone created by microbial fermentation of plant lignans are positively associated with protection against several hormone-associated cancers (e.g., breast, uterine, ovarian, and prostate) and were greatly increased in PD animals. Despite their relevance 
to the cancers mentioned, a significant association between these phytoestrogens and colorectal cancer has not been established $(277,278)$. Although characterization of the fatty acid profiles of the basal and dried plum diet revealed no significant differences, alterations in the relative concentration of numerous polyunsaturated fatty acids presented as fold changes were observed in the proximal and distal colon. For a given diet, all PUFAs detected tended to have higher concentrations in the proximal colon than the distal colon. However, dried plum rats had up to 3.3-fold higher levels of PUFAs (among other fatty acid compounds not presented) in their distal colons compared to rats consuming the BD. Various PUFAs have been shown to be major contributors to colon health in the prevention and treatment of colon cancer $(63,243,279-288)$. These observations are particularly intriguing considering the PICRUSt data suggesting increased fatty acid biosynthesis in the microbial metagenome of dried plum rats. However, whether these data reflect differences in production or uptake/utilization remains to be determined. Indeed, there is surging interest in the exploitation of microbial fatty acid biosynthesis from plant biomass for foodstuffs and bio-fuels (289-291). Dietary approaches that can take advantage of microbial fatty acid biosynthesis beyond short chain fatty acids, could be particularly relevant for colon health.

SCFAs are the primary product of microbial fermentation of dietary fiber and numerous anti-inflammatory and anti-cancer properties have been ascribed to them, as previously discussed. Prior to AOM treatment, animals consuming dried plum had lower levels of the branched chain fatty acids isobutyrate and isovalerate, which are produced from the breakdown of amino acids and the SCFA valerate, suggesting reductions in amino acid fermentation by the gut bacteria. As a result, the proportion of butyrate as a function of total SCFAs was higher in dried plum animals. This same trend was observed $48 \mathrm{hr}$ post-AOM treatment. The ability of 
dried plum to mitigate alterations in fecal SCFAs observed in BD+A animals following AOM exposure suggests retention of beneficial patterns of microbial metabolism during early disease stages. Specifically, BD+A animals had nearly a $100 \%$ increase in fecal acetate levels and a 9 $\mu \mathrm{mol} / \mathrm{g}$ increase in total fecal SCFAs after AOM treatment, whereas, PD+A animals showed no change in individual or total fecal SCFA levels. At 8 wk post-AOM, fecal SCFAs in BD+A animals returned to $\mathrm{BD}+\mathrm{S}$ animal levels, which contrasted with $\mathrm{PD}+\mathrm{A}$ animals whose SCFA profiles remained equivalent to $\mathrm{PD}+\mathrm{S}$ animals throughout the study. However, a major limitation of fecal SCFA measurement is that the observations do not reflect changes in SCFA production by bacteria nor uptake by the host. Quantification of the SCFA transport proteins in host tissue and/or quantification of critical microbial enzymes necessary to produce the SCFA metabolites could shed light on the nature of this observation.

It is clear that the microbiota contributes significantly to the health of the colon and risk of colon cancer and that the diet is a potent modifier of the intestinal microbe composition and metabolism. This study represents the early benefits of dried plum consumption on chemicallyinduced colon cancer from initiation to promotion but does not permit us to confirm a reduction in eventual tumor formation, which will be necessary to support subsequent human studies. AOM-induced colon cancer requires activation of the pre-carcinogen into the active mutagen by the liver and microbes, and because animals in this experiment were pre-fed with the experimental diets to permit microbial stabilization before initiation, it is possible that the metabolism and excretion of the carcinogen may have been affected. Nevertheless, this does not preclude an appreciation of the protection afforded by dried plum consumption. Dried plums may act directly on host tissues via the provision of native compounds to the host and indirectly through modification of the microbiota and their metabolism. As previously mentioned, the 
increasing incidence and mortality of distal colon cancers will require dietary chemoprevention strategies that can protect these sites. The enhanced influence of dried plum on the composition of the distal colon microbiota warrants further investigation into the bioavailability and bioaccessibility of dried plum-derived bioactive compounds and their microbial derivatives in the proximal and distal colon. Furthermore, because the diets utilized in this experiment were matched in terms of macronutrient profiles and ratio of soluble to insoluble fibers, we can with some confidence attribute these changes to the extra-nutritional components of the diet (e.g., the bioactive compounds). This ability of dietary bioactives to impact host health directly or through changes in the microbiota as prebiotics without dramatic modifications to the caloric profile of the diet, make bioactive-rich foods an attractive strategy for colon cancer prevention and treatment. Future studies will need to build on the observations made in this study and focus on elucidating the mechanisms of protection afforded by dried plums. Protein confirmation of affected genes (e.g., Slc16a1, Slc5a8, Nos2, Tlr2, ACADS, ACADM, etc.) in colon samples and the presence of microbial genes relevant to colon cancer (i.e., butyryl-CoA/acetyl-CoA transferase and phoshpotranbutrylase and butyrate kinase) (157) is warranted. An experiment in which the animals are introduced to the dried plum diet only after AOM treatment would determine whether the protection is related to the metabolism, efficacy, and excretion of the carcinogen, and, more importantly, whether dried plum consumption affords protection after an initiation event. Experiments employing germ free animals would help to determine to what extent the gut microbiota and its metabolism contribute to the protective effect afforded by dried plum consumption. Furthermore, it will be important to determine whether dried plum consumption has any effect on eventual tumor formation. Lastly, individual metabolites native to dried plum either alone or in combination with microbially-derived metabolites detected in this 
experiment could be used to conduct cell culture experiments for targeted or screening-based transcriptome analyses that could elucidate potential mechanisms for investigation in vivo. 


\section{CHAPTER III}

\section{DRIED PLUMS MODIFY COLON MICROBIOTA AND LUMINAL SCFA IN HZE- EXPOSED MICE}

\section{Introduction}

Exposure to ionizing radiation is a risk factor for development of colorectal cancer, and is known to affect the colon microbiota composition (104). Altered gut microbe compositions have been associated with colonic diseases including cancer and irritable bowel disease (23-25, 106, 292). Diet is a potent modifier of the microbiota and microbial metabolism, with the potential to attenuate harmful alterations resulting from exposure to ionizing radiation $(104,211,293)$. We have previously demonstrated that dried plum consumption in rats affects the composition and metabolism of bacteria in the colon, and that consumption of dried plums protects against chemically-induced colon carcinogenesis. Therefore, the objective of this experiment was to determine if consumption of a dried plum diet is able to prevent disruptions in colonic microbe composition and SCFA levels in mice exposed to high-charge, high-energy (HZE) ionizing radiation.

\section{Materials and Methods}

\section{$\underline{\text { Animals and diets }}$}

Adult mice (C57BL/6; $\mathrm{N}=40$ ), in a 2x2 factorial design, either received $1 \mathrm{~Gy}$ of ${ }^{56} \mathrm{Fe}$ whole-body radiation (600 MeV/n, $10 \mathrm{cGy} / \mathrm{min}$ ) or were sham irradiated (NSRL, Brookhaven National Laboratory, Upton, NY) and received a dried plum diet (DP+Sham or DP+Rad) containing a dried plum powder as $25 \%$ of total diet weight, or a control diet (AIN93M, Con+Sham or Con+Rad). Animals were sacrificed $30 \mathrm{~d}$ following radiation exposure and colonic 
luminal contents were collected and immediately snap frozen in liquid nitrogen. All animals were singly housed throughout the entirety of the experiment.

Luminal microbe profiling

Luminal bacterial profiling was conducted similarly to the methods previously described in Chapter II. Isolation of total DNA from stool samples was carried out as described (294-296). Total bacterial DNA was amplified using primers targeting the V1-V2 region of the 16S rRNA gene and overhang adapter sequences appended to the primer pair for compatibility with Illumina index and sequencing adapters (297). Master mixes used 2x KAPA HiFi HotStart Ready-mix (KAPA Biosystems, Wilmington, MA). Each 16S rRNA amplicon was purified using AMPure XP reagent (Beckman Coulter, Indianapolis, IN). In the next step each sample was amplified using a limited cycle PCR program, adding Illumina sequencing adapters and optional dual-index barcodes (index 1 (i7) and index 2 (i5)) (Illumina, San Diego, CA) to the amplicon target. The final libraries were again purified using AMPure XP reagent, quantified and normalized prior to pooling. The DNA library pool was then denatured with $\mathrm{NaOH}$, diluted with hybridization buffer and heat denatured before loading on the MiSeq reagent cartridge and on the MiSeq instrument (Illumina). Automated cluster generation and paired-end sequencing with dual reads was performed. Samples were rarefied to 1000 sequences/sample before downstream analyses.

Analysis of 16S rRNA amplicon-sequencing data was carried out using the QIIME pipeline (249) as described (294-298). Briefly, the combined raw sequencing data plus metadata was de-multiplexed and filtered for quality control. Sequences were grouped into Operational Taxonomic Units (OTUs) using UCLUST (251). After taxonomic assignation of OTUs, sequences were aligned and phylogenetic trees were built (299). OTU tables were used to 
perform alpha and beta diversity calculations, measurements that were used with sample metadata to create graphic visualizations for scientific interpretation. A combination of Unifrac significance and PCoA using Fast Unifrac (300) were done to compare samples based on relevant parameters. OTU sequences were aligned and OTU tables containing the counts of each OTU in each sample were used to calculate mean species diversity of each sample (alpha diversity) and the differentiation among samples (beta diversity). Alpha and beta diversity measures were used to calculate the Shannon-Weaver diversity index for each sample.

\section{$\underline{\text { SCFA analysis }}$}

The SCFA analyses were conducted using our standard protocols $(153,167)$. Fresh samples were flash frozen and stored at $-80^{\circ} \mathrm{C}$. Frozen samples were ground, mixed with an internal standard (2-ethylbutyric acid), and extracted in 70\% ethanol. Extracts were centrifuged and aliquots of supernatant removed and mixed with another internal standard (heptanoic acid) prior to injection onto a HP-FFAP column in a Varian 3900 GC (Agilent Technologies, Santa Clara, CA). SCFA concentrations in the samples were determined by comparison to a commercially available mix of standards.

\section{Results}

\section{Microbial profiling}

Overall, microbial analyses revealed that the dried plum powder diet increased the abundance of bacteria belonging to the phylum Actinobacteria $(P<0.0024)$, Tenericutes $(P<0.0196)$, and Verrucomicrobia $(P<0.0113)$, and reduced the proportion of sequences classified as unknown. Additionally, exposure to $1 \mathrm{~Gy}$ of ${ }^{56} \mathrm{Fe}$ ions increased bacteria belonging to Verrucomicrobia $(P=0.0432)$. However, interpretation of these statistical results is confounded by extreme variability observed almost exclusively in the DP+Rad group (Figure 9 and Table 

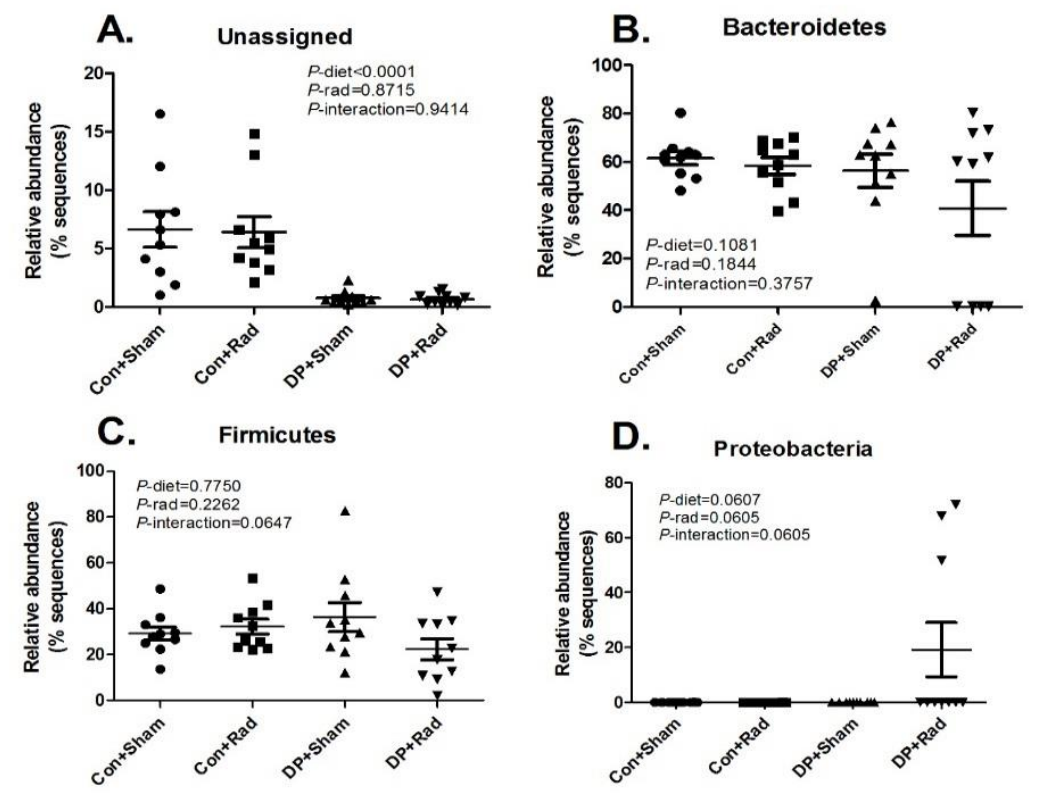

D. Proteobacteria
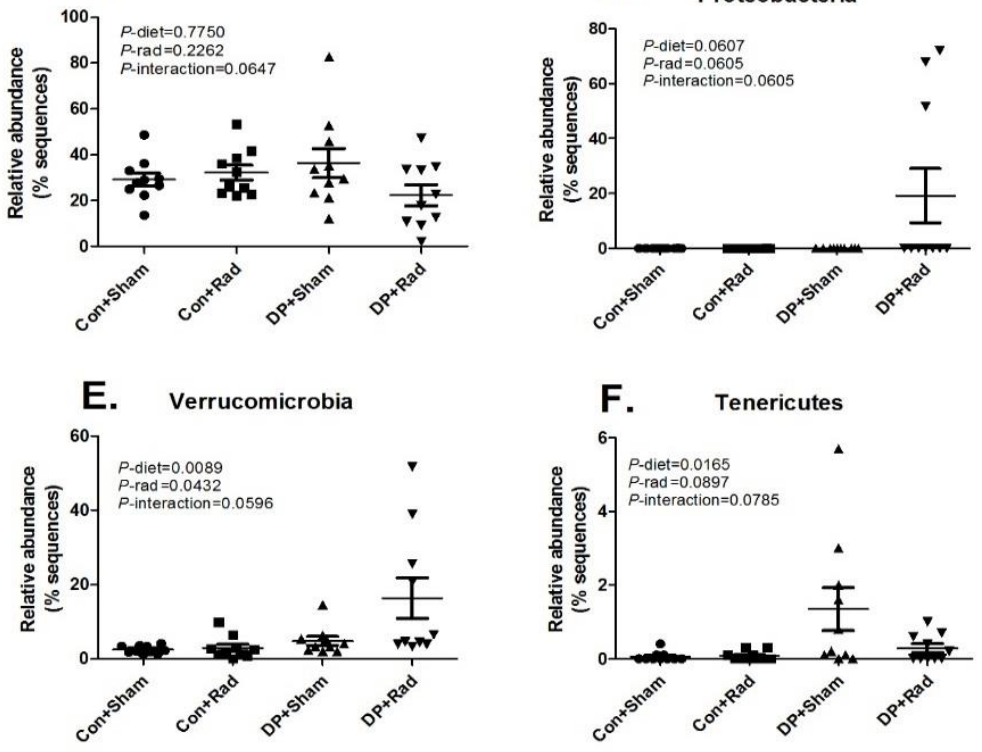

G. Actinobacteria

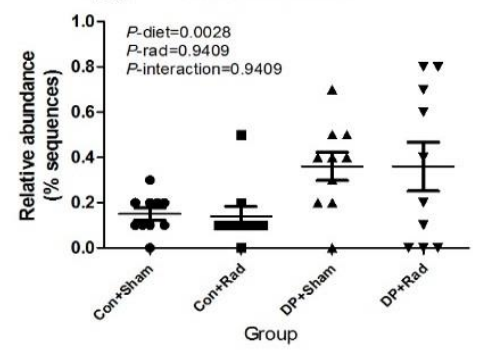

Figure 9. Relative abundance of bacterial phyla at $30 \mathrm{~d}$ post-IR in C57BL/6 mice consuming a control or dried plum diet and exposed to $1 \mathrm{~Gy}$ of ${ }^{56} \mathrm{Fe} \mathrm{HZE} \mathrm{radiation} \mathrm{or} \mathrm{sham} \mathrm{irradiated.}$ 
Table 11. Alterations in bacterial phyla and genera in C57BL/6 mice consuming a control or dried plum diet and exposed to 1 Gy of HZE radiation or sham irradiated.

\begin{tabular}{|c|c|c|c|c|c|c|c|}
\hline \multirow[b]{3}{*}{ Phylum } & \multicolumn{4}{|c|}{ Microbial Abundance (\% sequences) } & \multirow{2}{*}{\multicolumn{3}{|c|}{$P$-Values }} \\
\hline & \multicolumn{2}{|c|}{ Control Diet } & \multicolumn{2}{|c|}{ Dried Plum Diet } & & & \\
\hline & Sham & $1 \mathrm{~Gy} \mathrm{Fe}$ & Sham & $1 \mathrm{~Gy} \mathrm{Fe}$ & Diet & Radiation & Interaction \\
\hline Unassigned & $6.64 \pm 1.01^{\mathrm{a}}$ & $6.40 \pm 1.01^{\mathrm{a}}$ & $0.75 \pm 1.01^{\mathrm{b}}$ & $0.66 \pm 1.01^{\mathrm{b}}$ & $<0.0001$ & 0.8715 & 0.9414 \\
\hline Actinobacteria & $0.15 \pm 0.07^{\mathrm{a}}$ & $0.14 \pm 0.07^{\mathrm{a}}$ & $0.36 \pm 0.07^{\mathrm{b}}$ & $0.36 \pm 0.07^{\mathrm{b}}$ & 0.0028 & 0.9409 & 0.9409 \\
\hline Bacteroidetes & $61.44 \pm 6.90^{\mathrm{a}}$ & $58.29 \pm 6.90^{\mathrm{ab}}$ & $56.26 \pm 6.90^{\mathrm{ab}}$ & $40.73 \pm 6.90^{\mathrm{b}}$ & 0.1081 & 0.1844 & 0.3757 \\
\hline Firmicutes & $29.18 \pm 4.46^{\mathrm{ab}}$ & $32.19 \pm 4.46^{\mathrm{ab}}$ & $36.40 \pm 4.46^{\mathrm{a}}$ & $22.40 \pm 4.46^{\mathrm{b}}$ & 0.7750 & 0.2262 & 0.0647 \\
\hline Verrucomicrobia & $2.49 \pm 2.86^{\mathrm{a}}$ & $2.92 \pm 2.86^{\mathrm{a}}$ & $4.83 \pm 2.86^{\mathrm{a}}$ & $16.38 \pm 2.86^{\mathrm{b}}$ & 0.0089 & 0.0432 & 0.0596 \\
\hline Tenericutes & $0.06 \pm 0.30^{\mathrm{a}}$ & $0.08 \pm 0.30^{\mathrm{a}}$ & $1.35 \pm 0.30^{\mathrm{b}}$ & $0.29 \pm 0.30^{\mathrm{a}}$ & 0.0165 & 0.0897 & 0.0785 \\
\hline \multirow[t]{2}{*}{ Proteobacteria } & $0.01 \pm 4.94^{\mathrm{a}}$ & $0.01 \pm 4.94^{\mathrm{a}}$ & $0.00 \pm 4.94^{\mathrm{a}}$ & $19.16 \pm 4.94^{\mathrm{b}}$ & 0.0607 & 0.0605 & 0.0605 \\
\hline & \multicolumn{2}{|c|}{ Control Diet } & \multicolumn{2}{|c|}{ Dried Plum Diet } & \multicolumn{3}{|c|}{$P$-Values } \\
\hline Genus & Sham & $1 \mathrm{~Gy} \mathrm{Fe}$ & Sham & $1 \mathrm{~Gy} \mathrm{Fe}$ & Diet & Radiation & Interaction \\
\hline Unassigned & $6.64 \pm 1.01^{\mathrm{a}}$ & $6.39 \pm 1.01^{\mathrm{a}}$ & $0.65 \pm 1.01^{\mathrm{b}}$ & $0.76 \pm 1.01^{b}$ & $<0.0001$ & 0.8600 & 0.9445 \\
\hline$S 24-7$ & $61.43 \pm 6.90^{\mathrm{a}}$ & $58.27 \pm 6.90^{\mathrm{ab}}$ & $56.25 \pm 6.90^{\mathrm{ab}}$ & $40.72 \pm 6.90^{b}$ & 0.1082 & 0.1841 & 0.3761 \\
\hline Akkermansia & $2.50 \pm 2.86^{\mathrm{a}}$ & $2.92 \pm 2.86^{\mathrm{a}}$ & $4.84 \pm 2.86^{\mathrm{a}}$ & $16.39 \pm 2.86^{\mathrm{b}}$ & 0.0089 & 0.0433 & 0.0594 \\
\hline Enterobacteriaceae & $0.01 \pm 4.60^{\mathrm{a}}$ & $0.01 \pm 4.60^{\mathrm{a}}$ & $0.00 \pm 4.60^{\mathrm{a}}$ & $15.70 \pm 4.60^{\mathrm{b}}$ & 0.0967 & 0.0965 & 0.0963 \\
\hline Oscillospira & $4.17 \pm 0.71^{\mathrm{a}}$ & $4.86 \pm 0.71^{\mathrm{a}}$ & $2.85 \pm 0.71^{\mathrm{ab}}$ & $1.37 \pm 0.71^{b}$ & 0.0018 & 0.5848 & 0.1385 \\
\hline Ruminococcaceae & $3.72 \pm 0.56^{\mathrm{a}}$ & $3.88 \pm 0.56^{\mathrm{a}}$ & $2.71 \pm 0.56^{\mathrm{ab}}$ & $2.04 \pm 0.56^{\mathrm{b}}$ & 0.0151 & 0.6572 & 0.4627 \\
\hline Lachnospiraceae & $1.06 \pm 0.78^{\mathrm{a}}$ & $1.53 \pm 0.78^{\mathrm{a}}$ & $4.97 \pm 0.78^{b}$ & $2.74 \pm 0.78^{a}$ & 0.0022 & 0.2649 & 0.0900 \\
\hline Enterococcus & $0.00 \pm 3.42^{\mathrm{a}}$ & $0.00 \pm 3.42^{\mathrm{a}}$ & $5.99 \pm 3.42^{\mathrm{a}}$ & $3.34 \pm 3.42^{\mathrm{a}}$ & 0.1814 & 0.7005 & 0.7008 \\
\hline Lactobacillus & $1.49 \pm 0.35^{\mathrm{a}}$ & $1.42 \pm 0.35^{\mathrm{a}}$ & $0.09 \pm 0.35^{\mathrm{b}}$ & $0.15 \pm 0.35^{\mathrm{b}}$ & 0.0005 & 0.9803 & 0.8488 \\
\hline Ruminococcus & $0.10 \pm 0.01^{\mathrm{a}}$ & $0.12 \pm 0.01^{\mathrm{a}}$ & $0.05 \pm 0.01^{\mathrm{b}}$ & $0.02 \pm 0.01^{\mathrm{b}}$ & $<0.0001$ & 0.7699 & 0.0608 \\
\hline Turicibacter & $0.91 \pm 0.20^{\mathrm{a}}$ & $0.76 \pm 0.20^{\mathrm{a}}$ & $0.01 \pm 0.20^{\mathrm{b}}$ & $0.01 \pm 0.20^{\mathrm{b}}$ & 0.0002 & 0.7267 & 0.7289 \\
\hline Anaeroplasma & $0.01 \pm 0.30^{\mathrm{a}}$ & $0.01 \pm 0.30^{\mathrm{a}}$ & $1.35 \pm 0.30^{\mathrm{b}}$ & $0.25 \pm 0.30^{\mathrm{a}}$ & 0.0120 & 0.0756 & 0.0761 \\
\hline Coprococcus & $0.35 \pm 0.08^{\mathrm{ab}}$ & $0.40 \pm 0.08^{\mathrm{ab}}$ & $0.50 \pm 0.08^{\mathrm{a}}$ & $0.18 \pm 0.08^{b}$ & 0.6975 & 0.1047 & 0.0232 \\
\hline Clostridiaceae & $0.70 \pm 0.14^{\mathrm{a}}$ & $0.65 \pm 0.14^{\mathrm{a}}$ & $0.02 \pm 0.14^{\mathrm{b}}$ & $0.01 \pm 0.14^{\mathrm{b}}$ & $<0.0001$ & 0.8321 & 0.9012 \\
\hline Coriobacteriaceae & $0.00 \pm 0.06^{\mathrm{a}}$ & $0.00 \pm 0.06^{\mathrm{a}}$ & $0.31 \pm 0.06^{\mathrm{b}}$ & $0.32 \pm 0.06^{\mathrm{b}}$ & $<0.0001$ & 0.9345 & 0.9345 \\
\hline Uncategorized Clostridiales & $12.48 \pm 2.10^{\mathrm{a}}$ & $13.30 \pm 2.10^{\mathrm{a}}$ & $12.57 \pm 2.10^{\mathrm{a}}$ & $6.20 \pm 2.10^{\mathrm{b}}$ & 0.1035 & 0.1940 & 0.0953 \\
\hline
\end{tabular}

${ }_{\mathrm{a}, \mathrm{b}}$ Groups not sharing a common superscript letter are significant $(P<0.05)$. Data are LS means \pm SEM. Standard error uses a pooled estimate of error variance. All groups $n=10$. 
11). Four animals in the DP+Rad group had near complete loss of bacteria belonging to the Bacteroidetes phylum ( $\sim 0.1 \%$ of sequences assigned to Bacteroidetes), which comprised $\sim 61 \%$ of sequences in Con+Sham animals, $\sim 58 \%$ of sequences in Con+Rad animals, and $\sim 56 \%$ of sequences in DP+Sham animals. Of these four, three also had significant loss of Firmicutes bacteria (2-10\% of sequences). These three animals had 51.7-67.9\% of sequences identified as belonging to Proteobacteria and 20.8-38.9\% of sequences identified as Verrucomicrobia. The fourth DP+Rad animal exhibiting loss of Bacteroidetes had 51.8\% of sequences identified as Verrucomicrobia. Furthermore, one DP+Sham animal had near complete loss of Bacteroidetes ( $2.5 \%$ of sequences identified) in exchange for a significantly higher abundance of Firmicutes ( $82.6 \%$ of sequences) and Verrucomicrobia ( $14.5 \%$ versus $\sim 3.76 \%$ average for other animals in the DP+Sham group). As a result, these animals had extremely low bacterial diversity (Figure 10). Indices of species diversity (i.e., phylogenetic diversity (PD) whole tree and Shannon) and species richness (Chao1 and Observed species), all indicate substantial reductions in alpha diversity and evenness in these samples. All variables but PD whole tree passed the test for equal variances, but ANOVA revealed no significant differences between means.

At the genus level, the gut bacteria of mice were largely dominated by $S 24-7$ bacteria belonging to the Bacteroidetes phylum and to a lesser extent Clostridiales belonging to Firmicutes. The dramatic shifts in the three previously mentioned DP+Rad animals were the result of decreases in these dominant genera in exchange for, primarily, increases in Akkermansia (Verrucomicrobia phylum) and Enterobacteriaceae (Proteobacteria phylum). One DP+Rad animal had 33.9\% of sequences identified as Pseudomonas and another animal had a majority of sequences identified as Enterococcus (33.1\% of sequences). The remaining two DP+Rad 'outlier' animals were dominated almost exclusively by Akkermansia and 
Enterobacteriaceae. The single DP+Sham 'outlier' was largely dominated by Akkermansia (14\%), Enterococcus (59.8\%), and Lachnospiraceae (14.6\%).
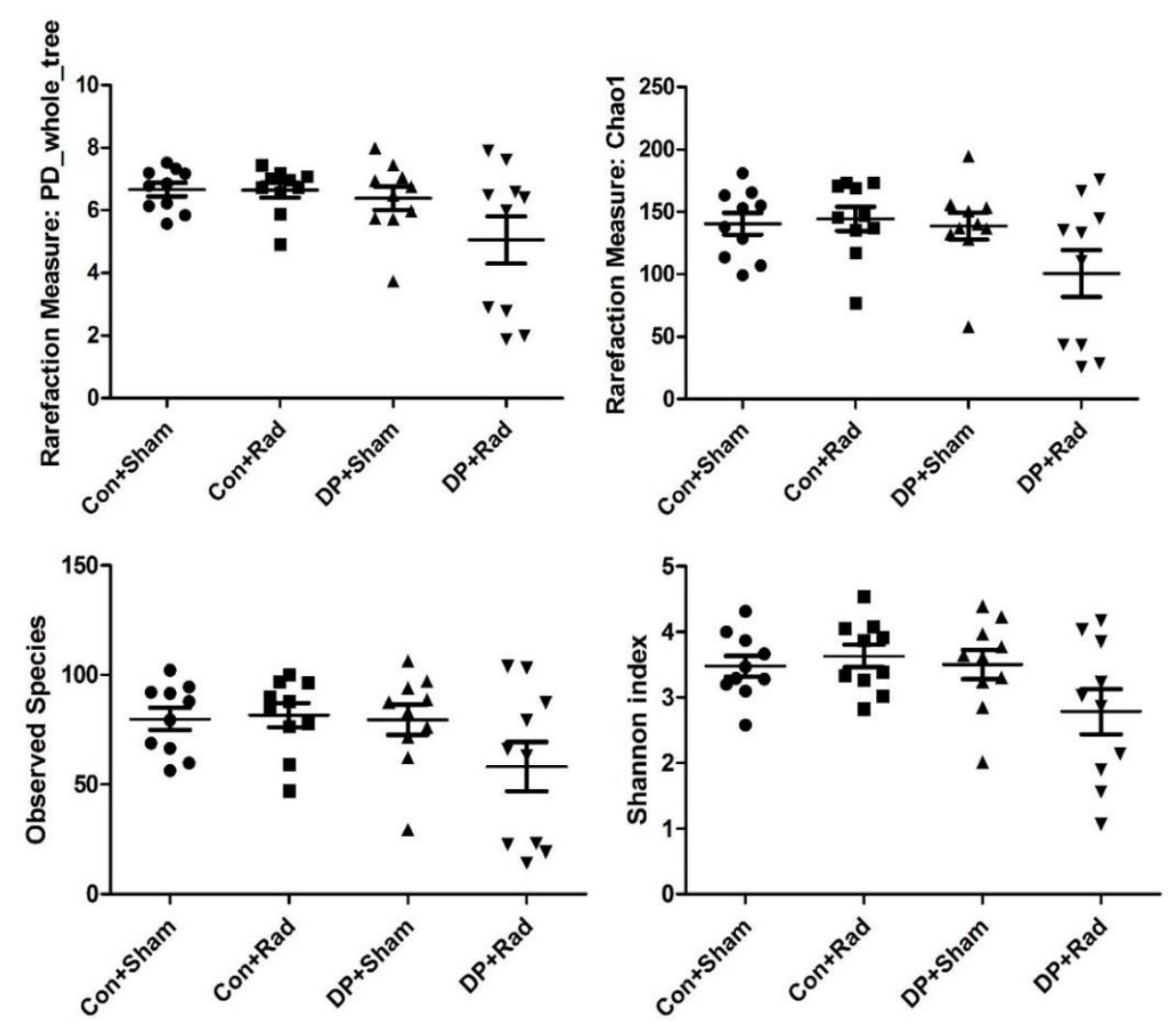

Figure 10. Alpha diversity indices of species diversity (PD whole tree and Shannon) and species richness (Chao1 and Observed species) at 1 month post-IR in C57BL/6 mice fed a control or dried plum diet and exposed to $1 \mathrm{~Gy}$ Fe ions or sham irradiated.

\section{$\underline{\text { SCFA analysis }}$}

Animals consuming the dried plum diet had significantly lower $(P<0.05)$ concentrations of all SCFA in the colonic lumen than control diet animals, except for acetate, which did not differ between diet groups $(P=0.0878)$ (Table 12). Consequently, dried plum animals had significantly lower total SCFA in their colonic lumens $(P=0.0069)$. Radiation had no effect on 
Table 12. Colon luminal SCFA concentrations in male C57BL/6J mice provided a control diet (AIN93M) or a diet containing dried plum at $25 \%$ of total diet weight and irradiated with $1 \mathrm{~Gy}$ Fe ions $(\sim 10 \mathrm{cGy} / \mathrm{min}, 600 \mathrm{MeV})$ or sham irradiated.

\begin{tabular}{|l|c|c|c|c|c|c|c|}
\hline & \multicolumn{2}{|c|}{ Control Diet } & \multicolumn{2}{c|}{ Dried Plum Diet } & \multicolumn{3}{c|}{ P-values } \\
\hline \multicolumn{1}{|c|}{ SCFA } & Sham & $\mathbf{1 ~ G y ~ F e}$ & Sham & $\mathbf{1 ~ G y ~ F e}$ & Diet & Radiation & Interaction \\
\hline Acetate & $12.44 \pm 1.30^{\mathrm{a}}$ & $11.46 \pm 1.30^{\mathrm{a}}$ & $10.19 \pm 1.30^{\mathrm{a}}$ & $9.15 \pm 1.30^{\mathrm{a}}$ & 0.0878 & 0.4414 & 0.9820 \\
\hline Propionate & $2.77 \pm 0.30^{\mathrm{a}}$ & $2.75 \pm 0.30^{\mathrm{a}}$ & $2.18 \pm 0.30^{\mathrm{a}}$ & $1.31 \pm 0.30^{\mathrm{b}}$ & $\mathbf{0 . 0 0 1 7}$ & 0.1472 & 0.1619 \\
\hline Butyrate & $2.85 \pm 0.21^{\mathrm{a}}$ & $2.32 \pm 0.21^{\mathrm{a}}$ & $1.50 \pm 0.21^{\mathrm{b}}$ & $1.35 \pm 0.21^{\mathrm{b}}$ & $<\mathbf{0 . 0 0 0 1}$ & 0.1070 & 0.3483 \\
\hline Isobutyrate & $0.27 \pm 0.03^{\mathrm{a}}$ & $0.23 \pm 0.03^{\mathrm{a}}$ & $0.10 \pm 0.03^{\mathrm{b}}$ & $0.10 \pm 0.03^{\mathrm{b}}$ & $<\mathbf{0 . 0 0 0 1}$ & 0.4774 & 0.3905 \\
\hline Valerate & $0.24 \pm 0.02^{\mathrm{a}}$ & $0.18 \pm 0.02^{\mathrm{b}}$ & $0.07 \pm 0.02^{\mathrm{c}}$ & $0.06 \pm 0.02^{\mathrm{c}}$ & $<\mathbf{0 . 0 0 0 1}$ & 0.0506 & 0.2249 \\
\hline Isovalerate & $0.26 \pm 0.03^{\mathrm{a}}$ & $0.23 \pm 0.03^{\mathrm{a}}$ & $0.11 \pm 0.03^{\mathrm{b}}$ & $0.08 \pm 0.03^{\mathrm{b}}$ & $<\mathbf{0 . 0 0 0 1}$ & 0.2548 & 0.8916 \\
\hline \multicolumn{1}{|c|}{ Total } & $18.83 \pm 1.71^{\mathrm{a}}$ & $17.16 \pm 1.71^{\mathrm{a}}$ & $14.14 \pm 1.71^{\mathrm{ab}}$ & $12.05 \pm 1.71^{\mathrm{b}}$ & $\mathbf{0 . 0 0 6 9}$ & 0.2796 & 0.9024 \\
\hline \% Acetate & $64.66 \pm 2.10^{\mathrm{a}}$ & $65.58 \pm 2.10^{\mathrm{a}}$ & $72.73 \pm 2.10^{\mathrm{b}}$ & $76.93 \pm 2.10^{\mathrm{b}}$ & $<\mathbf{0 . 0 0 0 1}$ & 0.2304 & 0.4401 \\
\hline \% Propionate & $15.04 \pm 1.32^{\mathrm{a}}$ & $16.18 \pm 1.32^{\mathrm{a}}$ & $14.90 \pm 1.32^{\mathrm{a}}$ & $10.23 \pm 1.32^{\mathrm{b}}$ & $\mathbf{0 . 0 2 7 5}$ & 0.1902 & $\mathbf{0 . 0 3 4 7}$ \\
\hline \% Butyrate & $16.01 \pm 1.21^{\mathrm{a}}$ & $14.25 \pm 1.21^{\mathrm{a}}$ & $10.48 \pm 1.21^{\mathrm{b}}$ & $11.02 \pm 1.21^{\mathrm{b}}$ & $\mathbf{0 . 0 0 0 9}$ & 0.6131 & 0.3466 \\
\hline \% Isobutyrate & $1.47 \pm 0.15^{\mathrm{a}}$ & $1.39 \pm 0.15^{\mathrm{a}}$ & $0.65 \pm 0.15^{\mathrm{b}}$ & $0.80 \pm 0.15^{\mathrm{b}}$ & $<\mathbf{0 . 0 0 0 1}$ & 0.8214 & 0.4494 \\
\hline \% Valerate & $1.35 \pm 0.12^{\mathrm{a}}$ & $1.15 \pm 0.12^{\mathrm{a}}$ & $0.49 \pm 0.12^{\mathrm{b}}$ & $0.42 \pm 0.12^{\mathrm{b}}$ & $<\mathbf{0 . 0 0 0 1}$ & 0.2582 & 0.5846 \\
\hline \% Isovalerate & $1.47 \pm 0.16^{\mathrm{a}}$ & $1.46 \pm 0.16^{\mathrm{a}}$ & $0.75 \pm 0.16^{\mathrm{b}}$ & $0.61 \pm 0.16^{\mathrm{b}}$ & $<\mathbf{0 . 0 0 0 1}$ & 0.6467 & 0.6976 \\
\hline
\end{tabular}

Data are least squares means \pm SEM. Standard error values are pooled estimates of variance. Values in the same row not sharing a common superscript are significantly different $(P<0.05)$. $\mathrm{N}=40$, group $\mathrm{n}=10$. 
individual or total SCFA concentrations irrespective of diet. In terms of percent contribution of each SCFA, the dried plum diet led to a greater percentage of acetate and lower percentage of the other SCFA as a function of total SCFA. The percent of propionate in the luminal contents from DP+Rad was significantly lower than all other treatment groups $(P<0.05)$.

\section{Discussion}

According to the USDA National Nutrient Database for Standard Reference, Release 23 (2010), a 40-gram serving of dried plums contains approximately $26 \mathrm{~g}$ carbohydrate ( $2.8 \mathrm{~g}$ fiber, $10 \mathrm{~g}$ glucose, $5 \mathrm{~g}$ fructose, and $2 \mathrm{~g}$ starch), $12 \mathrm{~g}$ water, and $1 \mathrm{~g}$ ash. Furthermore, dried plums contain a variety of compounds with known or suspected bioactive functions ascribed to them such as sorbitol, 4-hydroxymethylfurfural, and phenolic/polyphenolic compounds (194, 195, 259). Polyphenols are a major class of bioactive compounds found in dried plums, specifically neochlorogenic acid and chlorogenic acid, and the flavonol quercetin (194). Although most bacteria in the large intestine are saccharolytic (i.e., utilizing primarily carbohydrate/fiber), polyphenolic and phenolic compounds can also undergo metabolism by intestinal microbes to generate secondary metabolites (191). It has been determined that as much as $90 \%$ of polyphenol compounds can persist into the colon due to low bioavailability, implicating microbial metabolites of the polyphenols as the source of bioactivity $(301,302)$. It is important to emphasize, however, that this relationship is not unidirectional but rather a complex, multimicrobial, interconnected micro-ecosystem. Polyphenols can exert antimicrobial activities that shape the composition and, potentially, the functional profile of the intestinal microbiome (103, $134,192,193)$. Taken together, it is the direct actions of dietary polyphenols on host tissues and the microbiome, and the resulting bacterial metabolites of the polyphenols which can contribute to the phenotype observed following dried plum consumption. 
The work presented here indicates that the incorporation of a dried plum powder at $25 \%$ of total diet weight is capable of modifying the gut microbiome composition and luminal SCFA levels. Specifically, dried plum animals had significantly fewer bacteria classified as 'unknown' and instead had greater abundances of Verrucomicrobia, Tenericutes, and Actinobacteria. In this experiment, bacteria belonging to the genus Akkermansia were the sole contributor to the phylum Verrucomicrobia. Akkermansia muciniphila is a mucin-degrading bacteria shown to improve host metabolism and protect mice against diet-induced obesity $(303,304)$. Dietary polyphenols from concord grapes have recently been shown to promote the growth of Akkermansia muciniphila in mice, mitigating several effects of a high fat diet (305). These findings are consistent with our data demonstrating that dried plums containing polyphenols increase the abundance of Akkermansia in the mouse colon.

Surprisingly, an effect of radiation on colon microbe composition was only observed for Verrucomicrobia (Akkermansia muciniphila), which was increased following HZE exposure. Interestingly, there is evidence of an interaction effect between the dried plum diet and HZE radiation exposure that drastically impacts the steady state bacterial composition in some animals. Body weight and food intake measurements revealed no differences between the uniquely affected animals and other animals in their respective groups (data not shown). Recently, Akkermansia was shown to be elevated in the colon of mice exposed to radiation along with Proteobacteria and transmission of radiated mouse microbiota into germ-free wild type mice rendered them susceptible to DSS-induced colitis (306). These changes occurred in concert with increased inflammation mediated by TNF $\alpha$ and IL-1 $\beta$ and tissue damage. However, investigators did not determine which specific bacteria were promotive of the inflammation and injury versus those capable of mitigate the harmful effects post-IR. Indeed, Akkermansia has 
been associated with wound healing and mitigation of colitis in other mouse models of inflammation (304, 307-310). Taken together these observations suggest that the consumption of polyphenolic compounds, which are known to promote the growth of beneficial Akkermansia in addition to their direct antioxidant activities, could mitigate IR-induced colonic damage and inflammation. Future experiments using tissue obtained from these animals at termination would help to reveal whether or not these changes in the microbiome are protective.

Mice consuming the dried plum diet had significantly lower individual (except for acetate) and total SCFA concentrations compared to control diet animals. Akkermansia have been identified as key propionate producing bacteria (303), although our data indicate a reduction in luminal SCFA levels in mice fed the dried plum diet. Despite this increase in Akkermansia, it is possible that the dried plum selects against the growth of other bacteria capable of producing SCFA. Additional work will be necessary to more directly assess if dried plum has any effect on the production of SCFA, such as quantifying microbial enzymes/genes responsible for SCFA production (i.e., butyryl-CoA:acetate CoA-transferase). Alternatively, bioactive components of dried plum or microbial derivatives thereof could regulate the uptake of SCFA by colonocytes (e.g., SCFA transporters slcl6al and slc5a8) in host tissues, thereby affecting the luminal concentration of SCFA. Previous work in our lab failed to demonstrate a significant change in the expression of SCFA transporters or short- and medium-chain acyldehydrogenases in response to a dried plum diet in rats (311). Nevertheless, there is potential for regulation of these enzymes at the protein level, which were not assessed in that experiment $(311,312)$.

Radiation exposure had no effect on luminal SCFA levels in this experiment. Previous work with rats exposed to fractionated cesium gamma rays to a cumulative dose of $3 \mathrm{~Gy}$, showed 
significant reductions in fecal isovaleric acid and total SCFA 24 hours after the final radiation bolus (104). Animals in our experiment were exposed to a single acute dose of $1 \mathrm{~Gy} F e$ ions, which exert different biological effects compared to low LET radiation sources like gamma- and $\mathrm{x}$-rays. Furthermore, animals in this experiment were terminated at 30 days post-IR so it is possible that any effects on luminal SCFA by the radiation exposure may have been missed and/or resolved by the time animals were terminated. Conversely, diet was a strong modifier of luminal SCFA levels. Our previous work presented in Chapter II employing a dried plum puree diet in rats demonstrated that dried plum consumption significantly lowered fecal levels of several branched chain fatty acids, so the response observed in this experiment was not necessarily unexpected.

$\mathrm{DP}+$ Sham and DP+Rad animals exhibiting drastic alterations in their microbiota also had similar alterations in their SCFA levels versus other animals in their diet/treatment groups. Specifically, acetate, propionate, and butyrate were all markedly reduced in these animals, suggesting that decreased diversity of the microbiota as a result of interactions between the dried plum and radiation can affect luminal SCFA concentrations. Reduced SCFA levels and microbial diversity has been associated with intestinal diseases such as cancer and inflammation (100-102, 104), which may exacerbate the response to biological insults such as ionizing radiation. Additional assays with tissue samples from these animals will be necessary to determine whether or not the dried plum diet, exposure to ionizing radiation, and their interaction resulted in an observable phenotypic response that would permit further interpretation of the data presented here. High-LET, HZE (i.e., directly ionizing) radiation is known to produce complex DNA damage and to a lesser extent free radicals (i.e., indirectly ionizing radiation). Therefore, we would anticipate that dried plum, as a source of free radical scavenging antioxidants, would 
mitigate the indirectly ionizing component of exposure to HZE radiation. Biomarkers of oxidative stress and DNA damage (e.g., reactive oxygen species and 8-oxo-dG, respectively) have been shown to persist up to 1 year in the mouse intestine following ionizing radiation exposure (313).

Over the last 20 years, dried plums have generated significant attention for their role in promoting and preserving bone health, particularly in post-menopausal women and animal models thereof (314-319). More recently, the primary investigative group of the study presented here published strong data demonstrating that dried plum consumption can protect against bone loss caused by exposure to ionizing radiation, which they attributed in part to reductions in the expression of genes related to bone resorption (320). Radiation exposure of skeletal tissue facilitates rapid cancellous bone loss, increases in osteoclast number and activity, and production of reactive oxygen species (320). Dried plums contain essential and important vitamins and minerals, such as magnesium, boron, potassium, copper, and vitamin $\mathrm{K}$, which may contribute to the protective effects in bone (194); however, their phenolic compounds (e.g., chlorogenic acids, and neochlorogenic acids) are known to be potent superoxide radical scavengers (268). Importantly, in the experiment by Schreurs et al., an antioxidant cocktail diet containing 5 antioxidants (ascorbic acid, $\mathrm{N}$-acetyl cysteine, L-selenomethionine, dihydrolipoic acid and vitamin E), failed to prevent the effects of radiation on the expression of osteoclast related genes and subsequent bone loss (320). Interestingly, a cursory examination of the animals with uniquely altered microbial composition had similarly unique responses in bone health parameters (e.g., osteoblast colony numbers, percent bone mineralization, and bone mineral density; data not shown). These observations suggest that the alterations observed in the gut microbiota 
composition resulting from the interaction of dried plum and radiation exposure may have important implications beyond the intestines.

It is evident that dried plum is a strong modifier of the gut microbiota composition and luminal SCFA levels. Although there were only a few alterations observed in response to radiation exposure, it is possible that these changes are 1) masked by the greater effect of diet, 2) are transient effects that may resolve before 30 days post-IR, and/or 3) are too subtle to be detected with our sample size. Radiation is known to impact the composition of the gut microbiota, and certain microbes are known to be more radioresistant than others just as different mammalian cell populations can be more radiosensitive than others (321-324). In this experiment, radiation was only found to affect the abundance of Akkermansia, which was increased in response to exposure. However, these changes were seen only in $40 \%$ of DP+Rad and $10 \%$ of DP+Sham mice. We currently have no clear explanation for this observed phenomenon, but an interaction of dried plum and radiation is suggested. Animals were singly housed throughout the experiment which removes the potential for cage effects, and these animals were comparable to other animals in their diet/treatment groups with respect to diet intake and body weight. It is not well understood whether the impact of radiation on gut microbiota is stochastic (occurring over time without a threshold) or deterministic (occurring within a short time at a given threshold) and more research is needed to determine what the threshold for effect could be and how long the effect(s) could persist. Indeed, this is likely to be species (bacterial and host) dependent, and heavily impacted by diet. As seen in this experiment, a diet supplemented with dried plum powder is a much more 'potent' modifier of the gut microbiota composition and SCFA compared to HZE radiation expsoure. Without a greater understanding of functional changes in the microbiota and host tissues, it is difficult to derive 
any information regarding risk and risk mitigation from this experiment. Also, because the dried plum diet was a standard AIN-93M diet supplemented with a dried plum powder (25\% by weight), which was not analyzed for macronutrient content, it will be impossible to determine whether these alterations are primarily due to unique components of the dried plum (i.e., bioactives and fiber profile), or differences in macronutrient and caloric content. As diet is such a strong modifier of the gut microbiota and many bacteria are known to have substrate preferences, it will be important to employ these controls in future experiments. 


\section{CHAPTER IV}

\section{POLYPHENOL-RICH SUMAC SORGHUM DIETARY INTERVENTION ALTERS COLON MICROBIOTA AND PLASMA METABOLITES IN HUMAN SUBJECTS}

\section{Introduction}

The prevalence of overweight and obesity continues to increase dramatically, particularly in developed nations where the incidence of metabolic syndrome continues to rise in parallel (325). Metabolic syndrome is characterized by a constellation of factors known to increase the risk of heart disease, cancer, diabetes, and other chronic diseases $(326,327)$. The main risk factors include: abdominal obesity, high triglyceride and fasting glucose levels, low HDL cholesterol level, and high blood pressure (325). Furthermore, research has demonstrated that health complications associated with obesity include the manifestation of chronic, low-grade systemic inflammation (328-330). This elevated inflammatory tone is due in large part to the production of immune and inflammatory cytokines such as tumor necrosis factor (TNF- $\alpha$ ) and interleukin-6 (IL-6) produced in metabolically active adipose tissue depots (330); however, the contribution of diet and gut microbiota to systemic inflammation is less understood.

Gut bacteria thrive in the anaerobic environment of the intestinal tract and rely on dietary constituents that remain in the gut due to a lack of host digestive capacity or malabsorption. Gut bacteria exert a variety of functions capable of affecting host health including the digestion and metabolism of luminal contents and stimulation of the host immune system (331). One important function of certain gut microbes is the production of SCFA via fermentation of dietary fiber. SCFAs can be absorbed by the cells of the colonic epithelium, where they may serve as an energy source or a modifier of the epigenome $(156,158)$. Furthermore, the four-carbon SCFA 
butyrate is a potent mediator of the colonic inflammatory response involved in ulcerative colitis (UC) and colon cancer (159), and has been shown to improve insulin sensitivity and increase energy expenditure in mice (332). Indeed, there is growing evidence supporting the role of gut microbiota as a contributor to host metabolism and health (333-335).

The composition of the gut microbiota has been shown to differ between healthy and diseased individuals; a phenomena referred to as 'dysbiosis' (23). In particular, dysbiosis has been observed in concordance with obesity $(333,336,337)$, intestinal inflammation $(24,101)$, and colorectal cancer $(23,28,338)$ and is often broadly characterized by a high Firmicutes to Bacteroidetes ratio $(339,340)$. Therefore, modulation of the gut bacteria via easily achievable dietary interventions may provide an effective means for metabolic disease treatment and prevention in the general population.

Bioactive compounds are molecules capable of impacting the physiology of organisms consuming them. Plant foods (e.g. fruits, vegetables, grains, etc.) are a major source of dietary bioactive compounds and understanding the mechanisms by which their consumption can impact host physiology is a major focus in nutrition-based intervention strategies. Sorghum is an ancient grain predominantly grown in dry, arid climates and several varieties of sorghum contain different concentrations and classes of bioactive compounds. The sumac sorghum variety primarily contains polymers of flavonols (condensed tannins), which are also known as proanthocyanidins or procyanidins (179). Condensed tannins have high antioxidant activity and the sumac sorghum variety is among the highest in terms of polyphenol content $(179,341)$. Polyphenols, particularly condensed tannins, are poorly absorbed in their natural form due to their large size and their location within plant fiber matrices (342). For this reason, the bioactivefiber complexes of sorghum grain are believed to remain largely intact until they reach the large 
intestine where they can be metabolized by gut bacteria to form smaller bioactive metabolites (40, 343, 344). Additionally, there is evidence to suggest that many bioactive compounds may exert bactericidal activities further enhancing their ability to modulate bacterial populations (345).

We have previously found that sumac sorghum derived products have a low glycemic index, and when included in foods the bioactives contained in the sumac sorghum could reduce the rate of starch digestion $(346,347)$. Our research with rodent colitis models employing sorghum diets has shown changes in the composition of colon bacterial populations and fecal SCFA concentrations, suggesting that the bioactives in combination with the fiber matrix play a role in modulating gut microbiota metabolism $(103,212,245,348)$. The objective of the current study was to determine if a sorghum-based dietary intervention is able to affect fundamental host metabolic characteristics and/or modulate gut microbiota and their metabolism in overweight individuals at risk for developing metabolic syndrome.

\section{Materials and Methods}

\section{Study design and dietary intervention}

The procedures involved in this study were approved by the Institutional Review Boards of Texas A\&M University, Kansas State University and University of Nebraska and written informed consent was obtained from all subjects $(n=24)$ who participated. Males and females between the ages of 25-60 y, who were: nonsmokers; not taking medications for weight loss; not currently dieting for weight loss (weight had not changed more than $5 \mathrm{~kg}$ in either direction during the last 3 months); and had a BMI between 26 and 29 were recruited. Subjects must have been able to read, write and understand the English language. Subjects were excluded if they: did not regularly eat breakfast or snacks; had food allergies; had cardiovascular disease; were 
diabetic; had cancer in the 5 y prior to the study (except basal cell skin carcinoma); had renal or hepatic disease; had weight loss or gain of more than $5 \mathrm{~kg}$ in the previous 3 months (intentional or unintentional); had a history of drug or alcohol abuse in the previous 6 months; were taking weight loss, lipid-lowering, anti-hypertensive or anti-inflammatory steroid medications; had Crohn's disease, ulcerative colitis or other digestive disorders that interfere with digestion or absorption; women who were pregnant or lactating; women who did not have a regular menstrual cycle; were vegetarians; or people that consumed more than 15 grams per day of fiber. The 24 subjects ( $\mathrm{n}=8$ males and $\mathrm{n}=16$ females) recruited for the study were comparable in terms of age, weight, and BMI, differing only in their percent body fat (Table 13, $P<0.05)$.

Table 13. Baseline characteristics of all subjects $(\mathrm{n}=24)$ and separated by gender.

\begin{tabular}{|l|c|c|c|}
\hline \multicolumn{1}{|c|}{ Variable } & $\begin{array}{c}\text { All subjects } \\
(\mathbf{n = 2 4 )}\end{array}$ & $\begin{array}{c}\text { Male } \\
(\mathbf{n = 8})\end{array}$ & $\begin{array}{c}\text { Female } \\
(\mathbf{n = 1 6})\end{array}$ \\
\hline Age (years) & $41.3 \pm 13.2$ & $35.1 \pm 12.3^{\mathrm{a}}$ & $44.3 \pm 12.8^{\mathrm{a}}$ \\
\hline Weight $(\mathrm{kg})$ & $79.3 \pm 13.1$ & $85.8 \pm 13.4^{\mathrm{a}}$ & $76.0 \pm 12.0^{\mathrm{a}}$ \\
\hline BMI $\left(\mathrm{kg} / \mathrm{m}^{-2}\right)$ & $27.2 \pm 4.5$ & $27.3 \pm 4.4^{\mathrm{a}}$ & $27.2 \pm 4.6^{\mathrm{a}}$ \\
\hline Body fat $(\%)$ & $28.4 \pm 8.2$ & $22.9 \pm 7.2^{\mathrm{a}}$ & $31.2 \pm 7.4^{\mathrm{b}}$ \\
\hline
\end{tabular}

${ }^{\mathrm{a}, \mathrm{b}}$ For male and female columns, within a row, data without a common superscript differ $(P<0.05)$. Values are mean \pm s.d.

The study employed a randomized crossover design with two levels of sumac sorghum dietary intervention: low, $50 \mathrm{~g} /$ day and high, $100 \mathrm{~g} /$ day. The $50 \mathrm{~g} /$ day treatment was consumed at breakfast and the $100 \mathrm{~g} /$ day treatment was consumed in two sessions, one at breakfast and the other later in the day. The sumac sorghum intervention was an extruded cereal puff containing only $95 \%$ sumac flour and $5 \%$ sumac bran that resembled a cocoa puff in texture, color, and bowl life (i.e., how long the cereal retained texture when milk was added). Subjects were 
provided with an optional teaspoon sugar sachet to use if desired. A TX52 twin screw extruder (Wenger Mfg., Sabetha, KS) was used to process the extruded puffs. Subjects consumed each level of intervention for 4 weeks, separated by a 3-week washout period (Figure 11). Subjects were instructed not to change exercise regimen or diet, aside from the intervention. Samples and 24-hour diet records were collected at baseline and washout time points and following each intervention period. Samples for metabolite analysis were collected and body composition was assessed in the morning following a 10-hour overnight fast.

$\underline{\text { Diet records and nutrition analysis }}$

Three-day diet recalls were collected at baseline and leading up to the conclusion of each treatment period. Diet record data were analyzed using NDS software (349) to evaluate total calories, carbohydrate, fat, protein, cholesterol, fiber, etc. consumed by subjects.

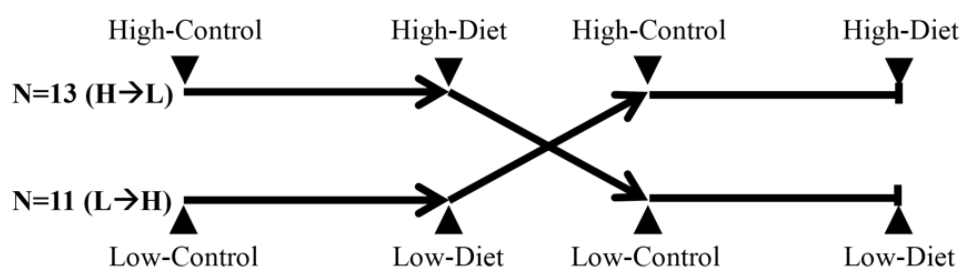

Figure 11. Experimental design and sample collection. $\boldsymbol{\nabla} / \mathbf{\Delta}$ indicates sample collection. Use of baseline samples and the crossover design allows subjects serve as their own controls.

\section{$\underline{\text { Microbial DNA analysis }}$}

Fecal samples were collected and frozen immediately in cryotubes the day before the last day, or the last day of, the treatment period. DNA was extracted and sequenced as previously described in Chapter II; sequencing was performed at University of Nebraska-Lincoln by Dr. Jens Walter (350, 351). Briefly, samples for microbiota analyses were processed within 2 hours 
of defecation. Samples were diluted in phosphate buffered saline (PBS) in a 1:10 ratio and stored in an ultra-low freezer $\left(-80^{\circ} \mathrm{C}\right)$ for genomic analysis. DNA was extracted from fecal samples to characterize the gut microbiome following the protocol described previously, using a combination of enzymatic cell-lysis (with lysozyme and proteinase-K), mechanical cell-lysis (bead-beating) and the QIAamp DNA Stool Mini Kit (Qiagen, Germany) (351). Once extracted, the DNA was stored frozen at $-80^{\circ} \mathrm{C}$ until sequencing was performed. The $\mathrm{V} 1-\mathrm{V} 3$ region of the bacterial 16S rRNA gene was amplified and sequenced by 454 pyrosequencing using a Roche Genome Sequencer GS-FLX with the Titanium platform (350). The first step after sequencing was to de-multiplex sequences by sample, and quality control the generated reads. The quality control parameters were the same as those used in a previous study (351). These steps were performed in the QIIME pipeline (qiime.org). After quality control, an average of $>3800$ sequences were obtained per sample, with an average length of 515 base pairs. These sequences were further used for taxonomic classification to depict the fecal microbial community. Essentially, two methods were used for this purpose. First, the Classifier web-tool of the Ribosomal Database Project (RDP http://rdp.cme.msu.edu/) was employed to taxonomically classify sequences from the phylum to the genus level, and second, a non-taxonomic-based approach was used to assign the sequences to Operational Taxonomic Units (OTUs) (97\% similarity), comparable to species-level bins. OTU-picking was performed using the UCLUST algorithm in QIIME (qiime.org).

To standardize the resulting taxonomic data, abundance of bacterial taxa was expressed as percent abundance with respect to the total number of sequences in the sample. These proportions were subjected to statistical analysis described below. 


\section{$\underline{\text { SCFA analysis }}$}

SCFA concentrations from fecal isolates were determined as previously described (153, 167). Briefly, fresh fecal samples were collected immediately after defecation, placed into cryovials and frozen, followed by storage at $-80^{\circ} \mathrm{C}$. Frozen samples were ground, mixed with an internal standard (2-ethylbutyric acid), and extracted in 70\% ethanol. The extract was centrifuged and the supernatant removed and mixed with second standard (heptanoic acid) just prior to injection onto a HP-FFAP (Agilent Technologies) column in a Varian 3900 GC. Concentrations were calculated based on comparison of peak areas with those produced by a commercially available mix of standards.

\section{$\underline{\text { Plasma metabolomics }}$}

Plasma was isolated from whole blood samples for metabolomic analyses in cooperation with Metabolon (Metabolon, Inc., Durham, NC). Briefly, profiling was performed using three independent platforms: ultrahigh performance liquid chromatography/tandem mass spectrometry (UHPLC/MS/MS ${ }^{2}$ ) optimized for basic species, UHPLC/MS/MS ${ }^{2}$ optimized for acidic species, and gas chromatography/mass spectrometry (GC/MS) (253). The original scale data, provided by Metabolon, was normalized in terms of raw area counts without any imputation of missing values.

$\underline{\text { Fecal metabolomics }}$

Frozen powdered fecal samples were extracted with a methanol:chloroform:water based extraction method as previously described (352). Briefly, $800 \mu \mathrm{L}$ of ice cold methanol:chloroform (1:1, v:v) was added to samples in a CK-Mix lysing kit tube (Bertin, Rockville, MD). Sample material was extracted on a Precellys 24 (Bertin) tissue homogenizer for $30 \mathrm{sec}$ at a speed of 6000 . The supernatant was collected and samples were homogenized a 
second time with $800 \mu \mathrm{L}$ of ice cold methanol:water. Six hundred microliters of ice cold water was added to the supernatant and vortexed for 30 seconds. Samples were centrifuged and the aqueous upper phase was collected. The aqueous layer was filtered through a $0.2 \mu \mathrm{m}$ nylon filter. Five hundred microliters of the filtered aqueous phase was then passed through a $3 \mathrm{kDa}$ cutoff column (Millipore, Burlington, MA) and the flow through was collected and lyophilized to dryness. Samples were resuspended in $50 \mu \mathrm{L}$ methanol:water (1:1, v:v) for analysis.

The target compounds in samples were detected and quantified on a triple quadrupole mass spectrometer (Quantiva, ThermoScientific, Waltham, MA) coupled to a binary pump HPLC (UltiMate 3000, ThermoScientific). MS parameters were optimized for the target compound under direct infusion at $5 \mu \mathrm{L} \mathrm{min}{ }^{-1}$ to identify the SRM transitions (precursor/product fragment ion pair) with the highest intensity (Table 14). Samples were maintained at $4^{\circ} \mathrm{C}$ on an autosampler before injection. The injection volume was $10 \mu \mathrm{L}$. Chromatographic separation was achieved on a hydrophilic interaction column (Luna $5 \mu \mathrm{m} \mathrm{NH} 2100 \AA 250 \times 2 \mathrm{~mm}$, Phenomenex, Torrance, CA) using a solvent gradient method (353). Solvent A was an ammonium acetate $(20 \mathrm{mM})$ solution in water with $5 \%$ acetonitrile $(\mathrm{v} / \mathrm{v})$. The $\mathrm{pH}$ of solvent A was adjusted to 9.5 immediately before analysis using ammonium hydroxide. Solvent B was pure acetonitrile. Table 15 describes the relevant literature information and rationale for the measurement of the metabolites of interest.

\section{$\underline{\text { Statistical analyses }}$}

Diet records, body composition, and glucose and lipid metabolism biomarkers (measured by collaborators), were analyzed with a one-way ANOVA with repeated measures. A $P$-value of $\leq 0.05$ was considered significant unless otherwise stated. 
Table 14. Quantitative SRM Transitions for compounds analyzed in fecal samples.

\begin{tabular}{|l|l|c|c|c|c|}
\hline \multicolumn{1}{|c|}{ Compound } & Polarity & $\begin{array}{c}\text { Precursor } \\
(\mathbf{m} / \mathbf{z})\end{array}$ & \multicolumn{1}{c|}{$\begin{array}{c}\text { Product } \\
(\mathbf{m} / \mathbf{z})\end{array}$} & $\begin{array}{c}\text { Collision } \\
\text { Energy (V) }\end{array}$ & $\begin{array}{c}\text { RF Lens } \\
(\mathbf{V})\end{array}$ \\
\hline Catechol & Negative & 109.1 & 91.1 & 20.0 & 56.9 \\
\hline Benzoic acid & Negative & 121.1 & 77.1 & 10.3 & 34.9 \\
\hline Trans-cinnamic acid & Negative & 147.1 & 103.1 & 12.7 & 40.6 \\
\hline 3-phenylpropionic acid & Negative & 149.1 & 105.1 & 10.3 & 44.3 \\
\hline 3-(3-hydroxyphenol)propionic acid & Negative & 165.1 & 121.1 & 10.3 & 48.3 \\
\hline Indole-3-propionic acid & Negative & 188.1 & 59.1 & 15.7 & 50.3 \\
\hline Quinic acid & Negative & 191.1 & 85.1 & 21.1 & 61.4 \\
\hline Cinnamoylglycine & Negative & 204.1 & 160.1 & 10.3 & 48.0 \\
\hline Indoxyl sulphate & Negative & 212.1 & 80.0 & 23.0 & 53.5 \\
\hline
\end{tabular}

Initial analysis of the fecal microbiota and plasma metabolite data revealed no significant dose response effects between the two levels of sumac sorghum intervention. For this reason, the effect of treatment (i.e., mean change in response to sumac sorghum treatment; $\frac{\Delta H+\Delta L}{2}$ ) was assessed for fecal microbes and plasma metabolites and is presented in this dissertation. The microbial data represents the proportion of total sequences in the sample that a given OTU was assigned. Each microbial taxon was assessed for normality. If the data was normally distributed, the raw proportion was analyzed. If not, then the data was normalized using the logit transformation $\left(\log \frac{(1+p)}{(1-p)}\right)$, where $\mathrm{p}$ is the proportion of the bacteria. The metabolomics data analyzed is 'scaled imputed' data provided by Metabolon Inc. and analyzed per their provided methods description (253). To generate this data, each biochemical value is normalized in terms of raw area counts, re-scaled to have median equal to 1 , and any missing values are imputed with the minimum (255). If the data were normally distributed, they were analyzed in the 'scaled imputed' format. If the data were not normally distributed, a log transformation was performed to achieve normality. If the log transformation was not sufficient to achieve normality, 
Table 15. Table of relevant literature and rationale supporting the investigation of specific metabolites in the human fecal samples.

\begin{tabular}{|c|c|c|}
\hline Metabolite & Rationale & References \\
\hline Quinic acid & $\begin{array}{l}\text { 1. Produced from chlorogenic acid by microbiota. } \\
\text { 2. Precursor to benzoic acid production by microbiota. } \\
\text { 3. Quinic acid moiety shown to be the major precursor of } \\
\text { hippuric acid. }\end{array}$ & $(354,355)$ \\
\hline Benzoic acid & $\begin{array}{l}\text { 1. Gut microbial metabolism of dietary aromatic compounds to } \\
\text { benzoate is first step in biosynthesis of hippurate. } \\
\text { 2. Benzoate may also be derived from other dietary sources; } \\
\text { however, we do not have data on dietary intake of benzoate } \\
\text { and no differences in benzoate observed in plasma samples. } \\
\text { 3. Major microbial metabolite generated which is then absorbed } \\
\text { and metabolized to form hippuric acid and 3- } \\
\text { hydroxyhippurate. }\end{array}$ & $(354,355)$ \\
\hline Cinnamic acid & $\begin{array}{l}\text { 1. Likely intermediate in the metabolism of phenylalanine to 3- } \\
\text { phenylpropionic acid. } \\
\text { 2. Cinnamic acid is formed from Phe by the enzyme } \\
\text { phenylalanine ammonia lyase. } \\
\text { 3. Cinnamic acid is converted in the mammalian body primarily } \\
\text { to hippuric acid and is excreted as such along with benzoic } \\
\text { acid. } \\
\text { 4. There are many cinnamic acid derivatives found naturally in } \\
\text { sorghum. }\end{array}$ & $(356-359)$ \\
\hline Cinnamoylglycine & $\begin{array}{l}\text { 1. Cinnamoylglycine, a glycine conjugate of cinnamic acid, is } \\
\text { known as a urinary metabolite in man (PMID: } 649712) \text {. } \\
\text { Whether it is formed de novo from plant cinnamate or is a } \\
\text { plant product excreted unchanged has not been conclusively } \\
\text { demonstrated. } \\
\text { 2. Not found when small quantities }(0.5-6 \mathrm{~g}) \text { of cinnamic acid } \\
\text { are fed to man, but by analogy with animal experiments may } \\
\text { be produced when much larger quantities are given. (PMID: } \\
\text { 6743769). } \\
\text { 3. Previously shown to be abundant in conventional mice but } \\
\text { only present in minimal concentrations in germ free mice. } \\
\text { 4. Cinnamoylglycine found to be a potential urinary biomarker } \\
\text { of colonization resistance by } C \text {. difficle. }\end{array}$ & (360) \\
\hline 3-phenylpropionic acid & $\begin{array}{l}\text { 1. Proanthocyanidin metabolite formed by human colonic } \\
\text { microflora from phenylalanine and proanthocyanidins } \\
\text { polymers. } \\
\text { 2. Despite an increase in serum levels of 3-phenylpropionic acid, } \\
\text { there was no difference in benzoic acid, suggesting its rapid } \\
\text { conversion into hippuric acid or conversion to alternate } \\
\text { glycine conjugated forms (i.e. phenylpropionylglycine). }\end{array}$ & $(190,356,357)$ \\
\hline 3-(3-hydroxyphenol)propionic acid & $\begin{array}{l}\text { 1. Metabolite derived from co-incubation of proanthocyanidins } \\
\text { with human colonic microflora. } \\
\text { 2. Upstream of benzoic acid and 3-hydroxyhippurate. }\end{array}$ & (190) \\
\hline Indoxyl sulfate & $\begin{array}{l}\text { 1. Derivative of microbial degradation of tryptophan found } \\
\text { significantly reduced in plasma of our subjects. }\end{array}$ & $(361,362)$ \\
\hline Indole-3-propionic acid & $\begin{array}{l}\text { 1. Major compound derived from microbial metabolism of } \\
\text { indole. } \\
\text { 2. Found only in the plasma of conventionally raised mice. } \\
\text { 3. Shown to be a powerful antioxidant. } \\
\text { 4. Shown to be produced by Clostridium sporogenes. } \\
\text { 5. IPA is rapidly cleared from the body, suggesting its presence } \\
\text { in serum relies on continuous production by the microbiota. }\end{array}$ & (356) \\
\hline
\end{tabular}


whichever form of the data was closest to a normal distribution was used for analysis.

T-tests on the treatment effect variables for microbes and metabolites were conducted and the means and standard errors for the variables are presented. Due to many hypotheses being tested simultaneously, multiple comparisons adjustments were made to the $P$-values generated from the t-tests. The $Q$-value is the $P$-value adjusted using the False Discovery Rate (FDR) approach. A $Q$-value $<0.1$ was considered significant unless otherwise stated.

For analysis of the fecal metabolomics, only subjects with metabolite signatures from baseline and post-high time points were included in the paired t-test. If the data was normally distributed a parametric t-test was performed. If the data was non-normal, a non-parametric t-test was performed. A $P$-value $<0.05$ was considered significant unless otherwise stated.

\section{Results}

Energy and nutrient intake, and body composition

Total energy intake was not significantly different between the treatment periods, however, there was an increase in fiber intake with each increase in cereal intake, and there was a significant increase in the intake of carbohydrate with the 50 and the $100 \mathrm{~g}$ of cereal consumed

(Table 16). Although subject carbohydrate intake was greater during the sorghum intervention, body composition and blood biochemistry appeared unaffected by the nearly 30 -g increase in carbohydrate intake (Table 17). At baseline, the average percent body fat was $28.4 \pm 8.2 \%$ (mean $\pm \mathrm{SE}$ ) and remained unchanged throughout the experiment despite the increase in carbohydrate intake and the minor increase in total energy intake during the treatment periods. 
Table 16. Data from 3-day diet records collected at baseline and at the end of each dietary intervention period.

\begin{tabular}{|l|c|c|c|}
\hline Macronutrient & Baseline & 50 g Cereal & 100 g Cereal \\
\hline Energy, kcal & $2204.0 \pm 72.8$ & $2187.6 \pm 126.1$ & $2241.8 \pm 95.8$ \\
\hline Fat, g & $86.7 \pm 3.7$ & $81.2 \pm 5.4$ & $82.9 \pm 4.8$ \\
\hline Saturated fat, g & $30.4 \pm 1.7$ & $27.3 \pm 2.2$ & $27.5 \pm 1.8$ \\
\hline Fiber, g & $18.0 \pm 1.4^{\mathrm{a}}$ & $21.9 \pm 1.2^{\mathrm{b}}$ & $25.4 \pm 1.1^{\mathrm{c}}$ \\
\hline Carbohydrate, g & $269.0 \pm 12.6^{\mathrm{a}}$ & $282.6 \pm 14.6^{\mathrm{b}}$ & $303.5 \pm 12.8^{\mathrm{c}}$ \\
\hline Protein, g & $80.2 \pm 3.3$ & $81.8 \pm 4.8$ & $80.2 \pm 4.0$ \\
\hline
\end{tabular}

a,b,c Within a row, data without a common superscript differ $(P<0.05)$. Data are means \pm SE.

Table 17. Blood glucose and lipid profiles prior to and after consuming the sumac cereal.

\begin{tabular}{|l|r|c|c|}
\hline \multicolumn{1}{|c|}{ Variable } & Baseline & 50 g Cereal & 100 g Cereal \\
\hline Body fat, $\%$ & $28.4 \pm 1.7$ & $28.6 \pm 1.6$ & $28.5 \pm 1.7$ \\
\hline Glucose, mg/dl, $(\mathrm{n}=23)$ & $89.4 \pm 4.1$ & $85.3 \pm 3.1$ & $86.4 \pm 3.2$ \\
\hline Total cholesterol, mg/dl, $(\mathrm{n}=23)$ & $169.1 \pm 7.6$ & $163.3 \pm 7.5$ & $172.8 \pm 10.0$ \\
\hline HDL cholesterol, mg/dl, $(\mathrm{n}=23)$ & $49.4 \pm 2.6$ & $46.1 \pm 2.6$ & $47.7 \pm 2.7$ \\
\hline LDL cholesterol, mg/dl, $(\mathrm{n}=18)$ & $108.4 \pm 6.9$ & $106.2 \pm 5.4$ & $114.4 \pm 7.1$ \\
\hline Non-HDL, $\mathrm{mg} / \mathrm{dl},(\mathrm{n}=23)$ & $124.0 \pm 7.6$ & $117.2 \pm 7.0$ & $125.2 \pm 9.0$ \\
\hline Total cholesterol/HDL, $(\mathrm{n}=23)$ & $3.6 \pm 0.2$ & $3.7 \pm 0.2$ & $3.8 \pm 0.3$ \\
\hline Triglycerides, $\mathrm{mg} / \mathrm{dl},(\mathrm{n}=18)$ & $110.8 \pm 16.2$ & $102.7 \pm 17.7$ & $108.8 \pm 19.9$ \\
\hline
\end{tabular}

Values are means \pm SE. No significant differences were observed for any variable.

$\underline{\text { Immunological marker analysis }}$

Undesirable microbial populations and/or damage to intestinal barrier integrity can

elevate circulating levels of lipopolysaccharide binding protein (LBP), which is associated with obesity-related insulin resistance (363). In addition, obesity has been linked to increases in other pro-inflammatory markers, including high-sensitive C-reactive protein (hs-CRP) and interleukin6 (IL-6), hallmarks of subclinical, chronic activation of the immune system. Plasma was analyzed to determine the levels of hs-CRP, IL-6, and LBP in plasma. We found no changes in the levels of these markers of inflammation in the subjects after consumption of either the 50 or $100 \mathrm{~g}$ of sumac sorghum cereal (Figure 12). 

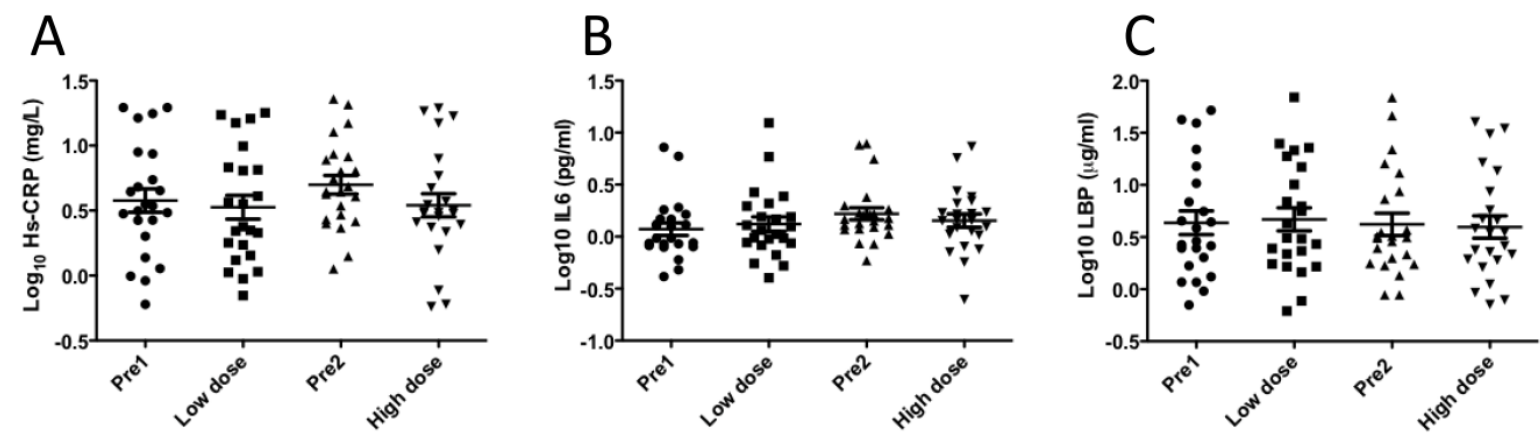

Figure 12. Circulating levels of (A) hs-CRP, (B) IL-6 and (C) LBP in subjects prior to and after consumption of either $50 \mathrm{~g}$ (low dose) or $100 \mathrm{~g}$ (high dose) of sumac sorghum breakfast cereal each day.

\section{$\underline{\text { Alterations in fecal microbiota }}$}

To determine the effect of the sumac sorghum puff cereal on the gut microbiota, fecal samples taken at each time point were subjected to DNA isolation and microbial amplification and sequencing of the bacterial $16 \mathrm{~S}$ rRNA gene at the V1-V3 regions. In total, there were 372,186 reads with an average number of reads per sample of $3,877 \pm 1,620$. The average length of reads used in the analyses was $490 \pm 14$ base pairs. Diversity analyses were calculated based on 2,000 sequences per sample to avoid bias in the number of sequences. With respect to alpha diversity (i.e., diversity within samples), there were no significant differences observed for Chao1, Shannon, or Simpson diversity indices or number of observed OTUs between treatment groups (Figure 13). Beta-diversity (i.e., diversity between samples) plots showed no significant clustering between treatments (Figure 14).

Our initial statistical analyses revealed no evidence of a dose response. Therefore, a treatment effect was calculated for the microbial taxa which averaged the response to the low and high sumac sorghum interventions. Significant alterations in microbes in response to sumac sorghum treatment are presented in Table 18 along with their $P$ - and $Q$-values. The sumac 

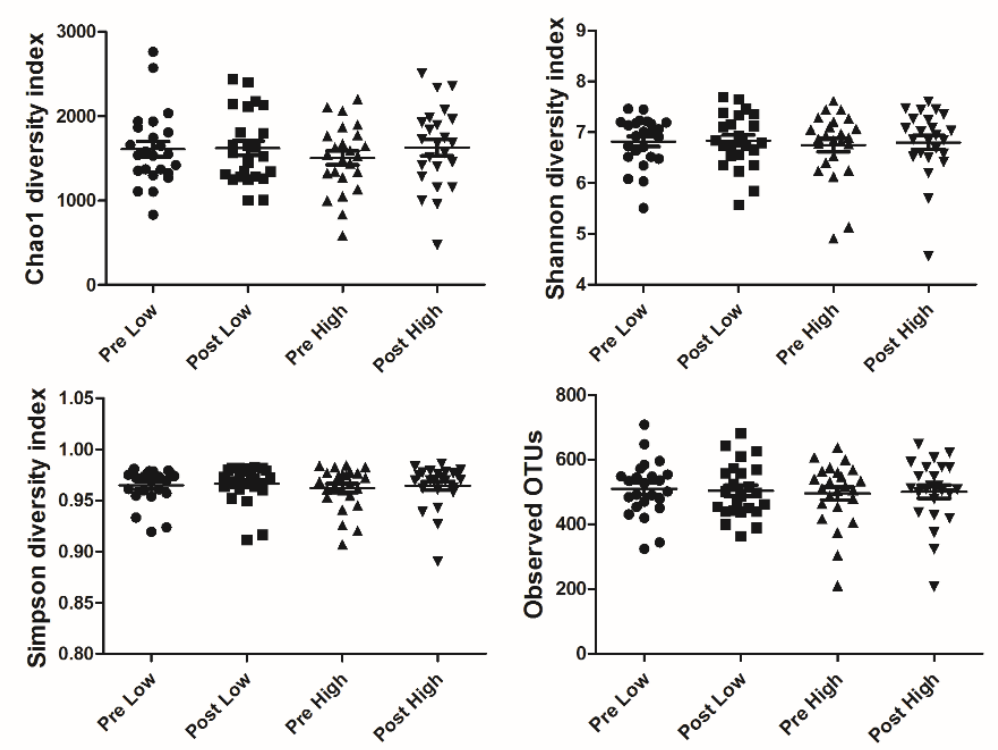

Figure 13. Alpha diversity estimates at Pre-Low, Post-Low, Pre-High, and Post-High sampling time points. No significant differences were observed between groups at any time point.

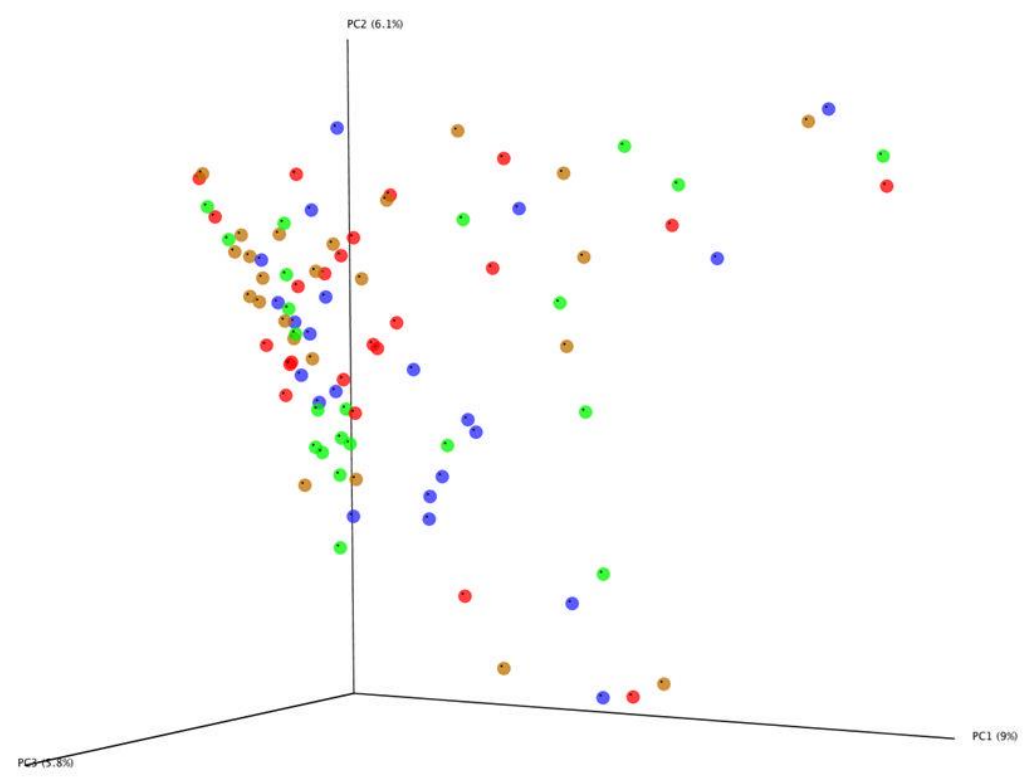

Figure 14. Beta-diversity principal components plot employing Bray Curtis dissimilarity for distance calculation between samples. Color coding by treatment: Pre1-red, Pre2-orange, Post High-blue, Post Low-green. No clustering by treatment was observed. 
sorghum intervention reduced the relative abundance of bacteria belonging to Firmicutes ($3.72 \pm 1.3 \%, Q=0.05030)$ and increased the relative abundance of bacteria belonging to Verrucomicrobia $(4.30 \pm 1.62 \%, Q=0.05030)$. Within the Firmicutes phylum, the largest alterations were observed in the genera Lachnospiraceae incertae sedis $(-3.92 \pm 1.08 \%$, $Q=0.07560)$ and Faecalibacterium (3.09 $\pm 1.08 \%, Q=0.07560)$. Decreases, albeit nonsignificant,

were also observed for the genera Dorea $(\sim 1.3 \%, Q=0.29011)$ and Blautia $(\sim 3.3 \%, Q=0.36676)$, which contribute to the $\sim 9.7 \%$ decrease in the Lachnospiraceae family noted in Table 11 . The increase in the Verrucomicrobia phylum was primarily due to a $4.29 \pm 0.91 \%$ increase in the genus Akkermansia but this change was not significant after FDR correction $(Q=0.28460)$. $\underline{\text { Short chain fatty acids }}$

Baseline fecal short chain fatty acid (SCFA) concentrations exhibited a high degree of inter-subject variability, which could be attributed to differences in pre-treatment bacterial composition, diet, and/or SCFA transporter expression in the host. Total and individual SCFA were not different between baseline and post-washout time points (data not shown). Total fecal SCFA concentrations did not change significantly in response to either the high- or lower-level sorghum diets (Table 19). Similarly, fecal butyrate concentrations were not affected by consumption of the low or higher level of sumac sorghum cereals.

$\underline{\text { Plasma metabolomic profiling }}$

Metabolomic profiles were established for comparisons in human plasma samples to determine if sumac sorghum consumption had any impact on the presence of metabolites reaching systemic circulation. From the significantly affected metabolites, those related to 
Table 18. Changes in select microbial abundances in response to sumac sorghum intervention.

\begin{tabular}{|l|l|l|c|c|c|c|}
\hline \multicolumn{1}{|c|}{ Taxa } & Level & Transformation & $\begin{array}{c}\Delta \text { Treatment } \\
(\boldsymbol{\%})\end{array}$ & $\begin{array}{c}\text { S.E. } \\
(\boldsymbol{\%})\end{array}$ & $\begin{array}{c}\boldsymbol{P} \text { - } \\
\text { value }\end{array}$ & $\begin{array}{c}\boldsymbol{Q} \text { - } \\
\text { value }\end{array}$ \\
\hline Firmicutes & Phylum & original & -3.72 & 1.30 & 0.0088 & $\mathbf{0 . 0 5 0 3}$ \\
\hline Verrucomicrobia & Phylum & $\log ((1+\mathrm{p}) /(1-\mathrm{p}))$ & 4.30 & 1.62 & 0.0144 & $\mathbf{0 . 0 5 0 3}$ \\
\hline Clostridia & Class & original & -5.37 & 0.99 & 0.0000 & $\mathbf{0 . 0 0 0 3}$ \\
\hline Verrucomicrobiae & Class & $\log ((1+\mathrm{p}) /(1-\mathrm{p}))$ & 4.29 & 1.62 & 0.0144 & $\mathbf{0 . 0 8 8 0}$ \\
\hline Gammaproteobacteria & Class & $\log ((1+\mathrm{p}) /(1-\mathrm{p}))$ & 0.32 & 0.13 & 0.0203 & $\mathbf{0 . 0 8 8 0}$ \\
\hline Clostridiales & Order & original & -5.33 & 0.99 & 0.0000 & $\mathbf{0 . 0 0 0 3}$ \\
\hline Lachnospiraceae & Family & original & -9.69 & 1.34 & 0.0000 & $\mathbf{0 . 0 0 0 0}$ \\
\hline Ruminococcaceae & Family & original & 3.90 & 1.31 & 0.0067 & $\mathbf{0 . 0 8 7 2}$ \\
\hline Verrucomicrobiaceae & Family & $\log ((1+\mathrm{p}) /(1-\mathrm{p}))$ & 4.29 & 1.62 & 0.0144 & $\mathbf{0 . 0 9 8 2}$ \\
\hline Micrococcaceae & Family & $\log ((1+\mathrm{p}) /(1-\mathrm{p}))$ & 0.04 & 0.01 & 0.0151 & $\mathbf{0 . 0 9 8 2}$ \\
\hline Lachnospiracea incertae sedis & Genus & original & -3.92 & 1.08 & 0.0014 & $\mathbf{0 . 0 7 5 6}$ \\
\hline Faecalibacterium & Genus & original & 3.09 & 1.08 & 0.0024 & $\mathbf{0 . 0 7 5 6}$ \\
\hline Akkermansia & Genus & original & 4.29 & 0.91 & 0.0144 & 0.2846 \\
\hline
\end{tabular}

Table 19. Baseline concentrations and changes in response to low, high and the average treatment response for total and individual SCFA.

\begin{tabular}{|c|c|c|c|c|}
\hline SCFA & $\begin{array}{l}\text { Baseline conc. } \\
(\mu \mathrm{mol} / \mathrm{g} \text { wet } \mathrm{wt} .)\end{array}$ & $\Delta$ Low & $\Delta$ High & $\Delta$ Treatment \\
\hline Total SCFA & $120.95 \pm 9.18$ & $-10.05 \pm 14.30$ & $3.89 \pm 11.80$ & $-3.08 \pm 7.49$ \\
\hline Acetate & $44.40 \pm 4.09$ & $-3.20 \pm 6.00$ & $3.00 \pm 4.64$ & $-0.10 \pm 3.04$ \\
\hline Propionate & $22.50 \pm 2.33$ & $-5.57 \pm 4.78$ & $0.82 \pm 2.05$ & $-2.38 \pm 2.69$ \\
\hline Butyrate & $29.46 \pm 4.12$ & $1.13 \pm 3.80$ & $-1.29 \pm 3.48$ & $-0.08 \pm 1.83$ \\
\hline Valerate & $11.36 \pm 1.28$ & $-1.50 \pm 1.13$ & $0.80 \pm 1.67$ & $-0.35 \pm 0.96$ \\
\hline Isobutyrate & $3.88 \pm 0.44$ & $-0.74 \pm 0.52$ & $0.15 \pm 0.56$ & $-0.30 \pm 0.37$ \\
\hline Isovalerate & $9.35 \pm 0.83$ & $-0.48 \pm 1.66$ & $0.40 \pm 1.36$ & $-0.04 \pm 1.26$ \\
\hline
\end{tabular}

Data are means \pm SE. $N=24$. No differences in SCFA concentrations were detected ( $p>0.05$ ).

microbial metabolism of dietary phenols, aromatic amino acids were selected for presentation in

Figure 15 due to their relevance to our stated objective of characterizing metabolites derived

from microbial-metabolism. These metabolites are presented across all time points, with the

adjusted $P$-value (i.e., $Q$-value) for the treatment effect noted. The complete list of metabolites

significantly altered by sumac sorghum consumption is presented in Appendix Table 5. Sumac

sorghum intervention produced increases in a number of metabolites derived from microbial

metabolism of dietary polyphenols (e.g., proanthocyanidins and chlorogenic acid) and

phenylalanine, including: catechol sulfate $(Q=0.004), 3$-hydroxyhippuric acid $(Q=0.019), 4$ - 
hydroxyhippuric acid ( $Q=0.083$ ), cinnamoylglycine ( $Q=0.024)$, and 3-phenylpropionate $(Q=0.083)(190,354-356,358,360,364-367)$. Additionally, sumac sorghum consumption led to reduced levels of the gamma-glutamyl amino acids (GGAAs) gamma-glutamylvaline $(Q<0.001)$ and gamma-glutamylmethionine $(Q=0.019)$. These and other GGAAs are generated by gammaglutamyl transferase (GGT) and elevated GGT is a biomarker for metabolic syndrome and cardiovascular disease risk (368-371).
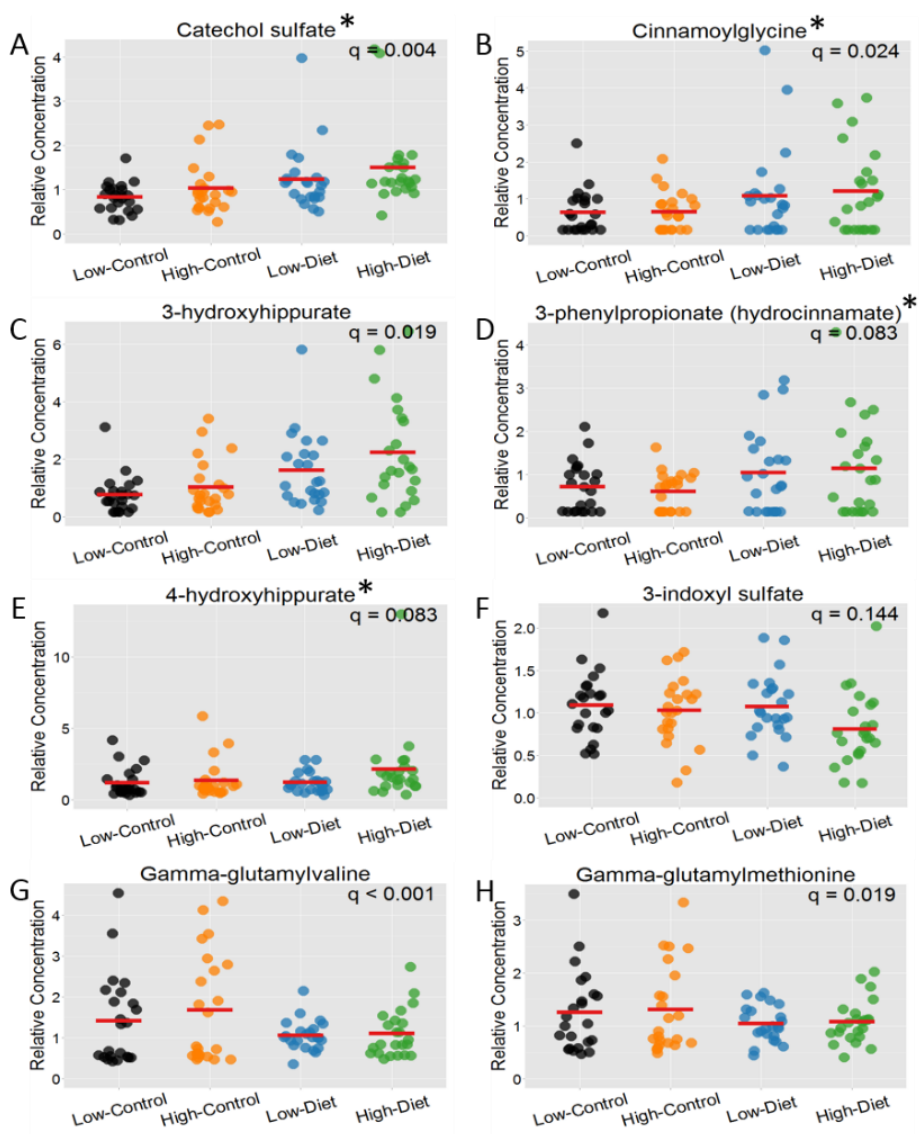

Figure 15. Relative concentrations of select plasma metabolites revealed by multi-platform mass spectrometry. A-E, metabolites generated from the microbial metabolism of dietary polyphenols and phenylalanine; F, derived from microbial metabolism of tryptophan; G-H, gamma-glutamyl amino acids involved in recycling oxidized glutathione. * indicates statistical analysis was performed on $\log$ transformed data. The treatment effect Q-value is also presented. 


\section{Fecal metabolite profiling}

As previously mentioned, many of the compounds detected in the plasma of subjects following sumac sorghum consumption are of particular interest because of their known microbial production. Furthermore, many of these metabolites are catabolites of proanthocyanidin polymers (e.g., condensed tannins), which are the major polyphenolic compounds in sumac sorghum. Therefore, we sought to determine the concentration of these and other associated metabolites in subject fecal samples. Figure 16 illustrates a conceptual pathway adapted from Wikoff et al. and Gonthier et al. $(355,356)$ for the production of the target metabolites based on the available literature presented in Table 9. Additionally, microbial metabolites of tryptophan such as indole, indoxyl sulfate, and indole-3-propionic acid have recently been investigated for their role in colonic health $(141,142,372-374)$. Unpublished work in our lab demonstrated reductions in fecal indole levels when rats consumed sumac sorghum. Although not significantly altered in subject plasma, 3-indoxyl sulfate tended to be lower in subjects following sumac sorghum consumption $(\mathrm{q}=0.14362)$.

For this reason, we also investigated the levels of two tryptophan/indole derivatives, 3indoxyl sulfate and indole-3-propionic acid, in baseline and post-high (100 g/day) subject fecal samples. The compounds of interest included: 3-(3-hydroxyphenol)propionic acid, 3phenylpropionic acid (aka hydrocinnamic acid), benzoic acid, quinic acid, indoxyl sulfate, indole-3-propionic acid, cinnamoylglycine, and cinnamic acid. Surprisingly, out of the 8 metabolites analyzed, only 5 were detected in our human fecal methanol:water extracts, with others being below the level of detection or quantification (Table 20). Because the statistical analysis of the data requires paired samples, only compounds detected in both samples for a given subject were used. Results of the paired t-tests are presented in Table 21. Compared to 
levels at baseline, following the high level of sumac sorghum consumption subjects had increased concentrations of 3-(3-hydroxyphenol)propionic acid ( 2.45 fold, $\mathrm{n}=13, P=0.000)$, 3phenylpropionic acid ( 2.25 fold, $\mathrm{n}=17, \mathrm{p}=0.004)$, and benzoic acid $(\sim 2.23$ fold, $\mathrm{n}=9, P=0.001)$. Quinic acid was decreased in subjects following the high sumac intervention period (35\% decrease, $\mathrm{n}=5, P=0.013)$.

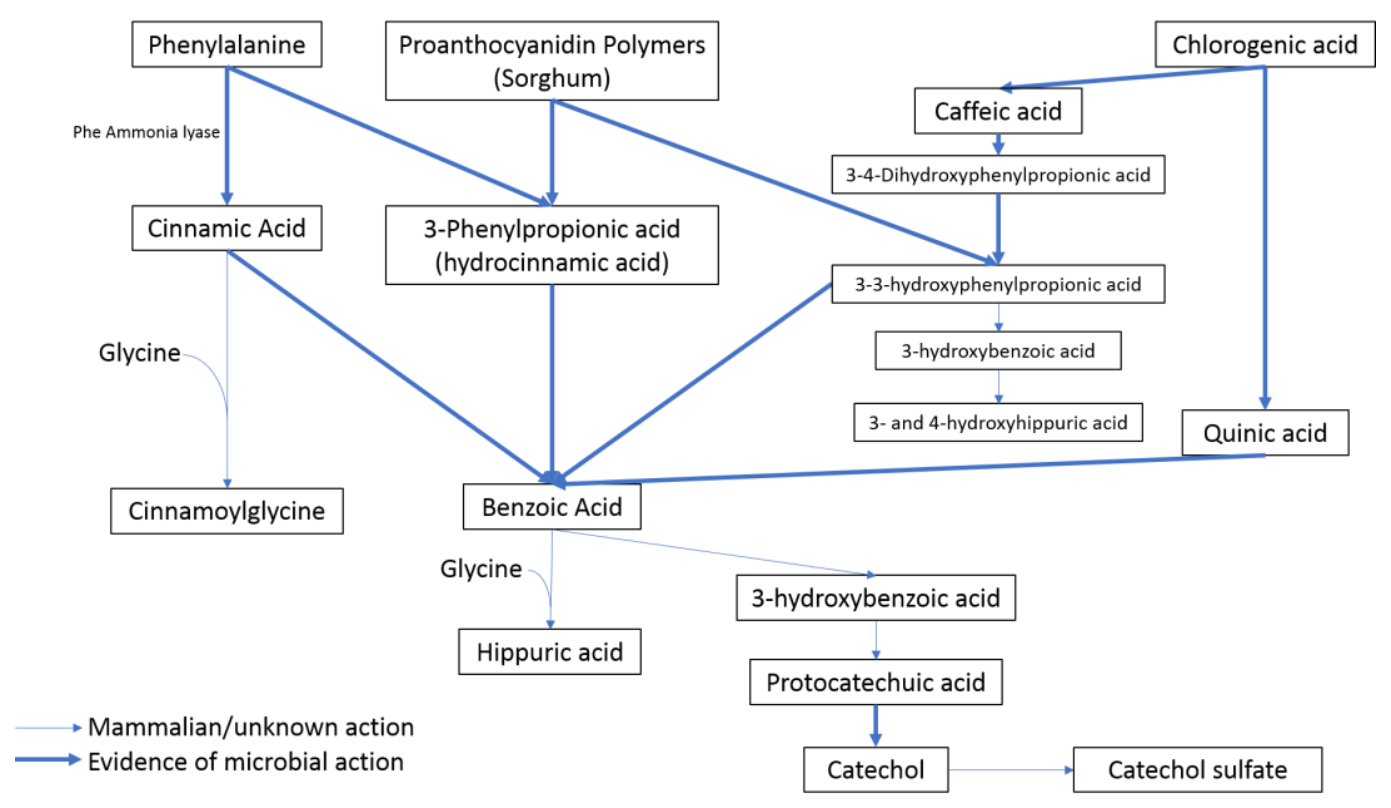

Figure 16. Conceptual diagram illustrating the production of metabolites from the dietary precursors: phenylalanine, proanthocyanidins, and chlorogenic acid. Adapted from Wikoff et al. and Gonthier et al. $(355,356)$.

\section{Discussion}

In this study we have demonstrated that through a minimal dietary change achieved by the consumption of extruded sorghum cereal, a source of dietary fiber containing complex polyphenols, it is possible to modulate the composition of fecal bacteria and to increase the 
relative concentrations of aromatic and phenolic compounds produced by microbial metabolism in host plasma and the intestinal lumen. Many of the putatively bioactive compounds in the diet

Table 20. Metabolites selected for targeted quantification in subject fecal samples at baseline and post-high time points.

\begin{tabular}{|l|l|c|c|c|c|c|}
\hline \multicolumn{1}{|c|}{ Compound } & CAS ID & $\begin{array}{c}\# \\
\text { samples } \\
\text { analyzed }\end{array}$ & $\begin{array}{c}\# \\
\text { samples } \\
\text { detected }\end{array}$ & $\begin{array}{c}\# \\
\text { BLOQ }^{\mathbf{1}}\end{array}$ & $\begin{array}{c}\text { \# } \\
\text { BLOD }^{\mathbf{2}}\end{array}$ & $\begin{array}{c}\text { \# Paired } \\
\text { samples } \\
\text { detected }\end{array}$ \\
\hline 3-(3-hydroxyphenol)propionic acid & $621-54-5$ & 47 & 34 & 1 & 12 & 13 \\
\hline 3-phenylpropionic acid & $501-52-0$ & 47 & 38 & 0 & 9 & 17 \\
\hline Benzoic acid & $65-85-0$ & 47 & 27 & 5 & 15 & 9 \\
\hline Quinic acid & $77-95-2$ & 47 & 17 & 9 & 21 & 5 \\
\hline Indoxyl sulphate & $2642-37-7$ & 47 & 7 & 1 & 39 & 0 \\
\hline Indole-3-propionic acid & $830-96-6$ & 47 & 0 & 0 & 47 & 0 \\
\hline Cinnamoylglycine & $16534-24-0$ & 47 & 0 & 0 & 47 & 0 \\
\hline Cinnamic acid & $140-10-3$ & 47 & 0 & 0 & 47 & 0 \\
\hline
\end{tabular}

${ }^{1}$ Below the Level of Quantification (BLOQ). Refers to when peak is detected but cannot be quantified because the signal to noise ratio is $<5 .{ }^{2}$ Below the Level of Detection (BLOD). Refers to when there is no visible peak for the sample.

Table 21. Paired t-test results for the targeted fecal metabolite quantification at baseline and post-high time points.

\begin{tabular}{|l|c|c|c|c|}
\hline \multicolumn{1}{|c|}{ Compound } & N & $\begin{array}{c}\text { Pre-Diet } \\
(\mathbf{u g} / \mathbf{m l})\end{array}$ & $\begin{array}{c}\text { Post-High } \\
(\mathbf{u g} / \mathbf{m l})\end{array}$ & $\boldsymbol{P}$-value \\
\hline 3-(3-hydroxyphenol)propionic acid & 13 & $13.26 \pm 4.35$ & $32.52 \pm 11.16$ & $0.000^{*}$ \\
\hline 3-phenylpropionic acid & 17 & $10.96 \pm 2.76$ & $24.72 \pm 3.93$ & 0.004 \\
\hline Benzoic acid & 9 & $0.39 \pm 0.15$ & $0.87 \pm 0.36$ & $0.001^{*}$ \\
\hline Quinic acid & 5 & $0.86 \pm 0.25$ & $0.30 \pm 0.07$ & $0.013^{*}$ \\
\hline
\end{tabular}

"Data for this compound was not normally distributed and a non-parametric t-test was performed using ranked data.

have low native bioavailability due to their polymerization and a lack of digestive capability by the host $(302,375)$. Many bioactive compounds are also held within plant fiber matrices and, therefore, have reduced bioaccessibility until microbial digestion of the carbohydrate latticework releases the compounds for further metabolism (375). Thus, the extent of bioavailability and bioaccessibility of bioactives in foods determines when, where, and to what extent a bioactive compound can be utilized/metabolized by the host, which can be dependent on the gut microbiota. By providing modest levels of sumac sorghum in the form of a breakfast cereal, we 
were able to modify the levels of microbially-derived metabolites in the blood and feces of our subjects. Many of the metabolites significantly affected by the dietary intervention have been identified as potentially relevant biomarkers for cardiovascular disease risk, impaired glucose tolerance, and metabolic syndrome $(255,368-371,376,377)$; however, their exact bioactive function(s) and mechanisms of action (if any) have yet to be determined. Experiments utilizing the microbially derived and parent compounds detected in our subjects following sumac sorghum consumption will be necessary to infer causal relationships beyond the correlative associations currently ascribed to these compounds.

Following sumac sorghum consumption, there was no difference in alpha-or betadiversity metrics for fecal microbiota. This observation is not uncommon in human dietary intervention studies as large inter-individual variation in microbial populations promotes clustering by subject rather than treatment (378). Subjects had decreased bacteria belonging to the Firmicutes phylum and increased bacteria belonging to the Verrucomicrobia phylum. The decrease in Firmicutes was largely attributed to a decrease in Lachnospiraceae incertae sedis, which can be more appropriately classified as bacteria of the Lachnospiraceae family of 'uncertain placement.' Lachnospiraceae bacteria are anaerobic bacteria belonging to the Clostridiales order and occur in the human and mammalian gut microbiota $(379,380)$. These bacteria have been linked to obesity and protection of colon cancer in humans, due in part to their production of butyrate; however, not all members of this family produce butyrate (381, 382). Feeding mice with a high fat diet increased the abundance of Lachnospiraceae bacteria, which accompanied an increase in $\beta$-catenin signaling and inflammation (383). Another study determined that intestinal colonization of germ free $o b / o b$ mice by a strain of Lachnospiraceae contributed to the development of diabetes (384). Unfortunately, we are not able to identify 
which particular members of the Lachnospiraceae family of bacteria were present in our human samples at this depth of sequencing; however, the association with this bacteria and its positive association with the aforementioned metabolic disorders suggests that longer term reductions in the bacterium could beneficially impact metabolic syndrome risk.

Conversely, sumac sorghum significantly increased the abundance of Faecalibacterium and tended to increase Akkermansia (4\% on average in response to sumac consumption). Faecalibacterium is a genus of bacteria with the sole known species, Faecalibacterium prausnitzii, whose depletion in the gut has been reported in several intestinal disorders (385). Faecalibacterium has been described as an anti-inflammatory, commensal bacterium that is negatively associated with colitis, and positively associated with maintenance of clinical remission in ulcerative colitis (386-389). Butyrate-dependent $(390,391)$ and -independent $(387$, 388) mechanisms have been proposed to explain the role of Faecalibacterium in colitis and in the promotion of intestinal barrier function and inflammation suppression. Consequently, Faecalibacterium, has been identified as a good candidate for clinical investigation as a probiotic (385). Akkermansia, although not statistically significant after FDR correction, displayed a tendency for increased abundance in subjects consuming sumac sorghum. There are only two known species of Akkermansia, Akkermansia muciniphila, which is found in the human an mammalian gut, and Akkermansia glycaniphila recently identified by Ouwerkerk et al. in reptiles (392). The former is a gram-negative, strict anaerobe, capable of utilizing mucins as a sole source of carbon and nitrogen (303). Similar to Faecalibacterium, Akkermansia muciniphila, has been associated with improved glucose tolerance, reduced adipose tissue inflammation, and improved gut barrier function $(308,393)$. Additionally, Akkermansia has been associated with attenuation of high fat diet-induced metabolic syndrome in C57BL/6J mice (305). Furthermore, 
there is evidence demonstrating that dietary polyphenols from various sources are able to promote the growth and abundance of Akkermansia in the gut $(305,307,309,393)$, which supports our finding that sumac sorghum containing polyphenols in the form of condensed tannins, can act as a prebiotic to increase the abundance of Akkermansia in the human gut. However, it is important to note that many of the associations discussed here have not been mechanistically established and more work will be necessary to determine if, and/or to what extent alterations in these microbes contribute to the improved host physiology. In experiments where dietary polyphenols are being studied, it will be important to dissect direct effects of the compounds on the host from those that are facilitated by microbial functions, such as metabolism of the native compounds into other bioactive metabolites. Nevertheless, further investigation of the mechanisms by which Faecalibacterium and Akkermansia can improve the constellation of risk factors related to metabolic syndrome and intestinal disease, especially in combination with dietary polyphenols as bioactive microbial substrates, is especially warranted.

Despite the alterations in putatively beneficial bacteria, we were not able to demonstrate significant changes in the risk factors associated with metabolic syndrome (e.g. BMI, cholesterol, fasting plasma glucose, etc.) over the short intervention period. It will be necessary to conduct longer dietary intervention periods in future experiments to determine if this pattern is retained over time, as there remains a potential for long term therapeutic effects. Of course, comprehensive studies at the level of the individual will be necessary to determine how lifestyle and genetic factors contribute to our endpoints. The microbiome is widely accepted as a major contributor to host health and changes in its composition following dietary interventions have been recently defined as 'rapid and reproducible' (293). However, disease phenotypes associated with abnormal metabolism such as cancer and diabetes can take years to develop, therefore, it 
isn't unreasonable to suggest that complete reprogramming of an unhealthy metabolic phenotype may take a similar length of time.

Although we were not able to show significant changes in fecal SCFA concentrations, it is important to note that the dietary fiber provided by sumac sorghum is primarily insoluble and, thus, is not as efficiently metabolized by the bacteria $(37,210,394)$. Furthermore, fecal concentrations of SCFA may not adequately reflect production by the bacteria or uptake by the host in vivo. For these reasons, we cannot conclude that the sorghum had any effect on the fermentative processes of the microbiota in our human subjects. Prior data in our animal models suggest that sumac consumption can reduce the level of excreted fecal butyrate, relative to cellulose fed control animals and animals consuming additional sorghum varieties (103, 212, 245, 348). Indeed, the microbial analyses indicate a significant increase in Faecalibacterium prausnitzii, a bacterium associated with relatively high levels of butyrate production as previously mentioned. Further work will be needed to determine if the rate of SCFA synthesis, utilization by the bacteria, or absorption by the host can be influenced by the sorghum cereal.

The ability of dietary polyphenols to exert bioactive functions is likely a combination of changes to the gut microbiome and direct effects of the native compounds directly on host cells and tissues. The former includes both changes to the composition and the metabolism of gut bacteria. In this experiment, we sought to characterize the plasma metabolome of subjects before and after the dietary intervention in order to identify the metabolic impact of sumac sorghum consumption. As anticipated, a number of biochemicals derived from microbial metabolism of plant phenols were altered in plasma samples following sumac sorghum consumption. Catechol sulfate, a sulfate-conjugated derivative of phenolic compounds, was increased in subject plasma after sumac sorghum consumption. Work in human subjects has shown it is increased in plasma 
after consumption of a mixed fruit/berry puree, reaching concentrations of 5 to $20 \mu \mathrm{m}$ (395). Another recent human experiment employing lean and obese subjects consuming mango demonstrated an inverse correlation between plasma concentrations of catechol sulfate with BMI, suggesting that increases in BMI are associated with reduced polyphenol absorption (396). Furthermore, catechol sulfate was positively associated with IL-10 production in both lean and obese subjects, suggesting it could have anti-inflammatory activity. Another compound derived from microbial metabolism of dietary phenols, 3-hydroxyhippurate, was increased in subject plasma following sumac sorghum consumption. 3-hydroxyhippurate is an aromatic acid found in urine and plasma of humans, and is derived from the microbial metabolism of dietary polyphenols, such as chlorogenic acid (189, 354, 355, 397-399). Decreased urinary levels of 3 hydroxyhippurate have been associated with impaired glucose tolerance (376). 4hydroxyhippurate was similarly increased in subjects following sumac consumption.

Two metabolites derived from aromatic amino acids (e.g., phenylalanine), cinnamoylglycine and 3-phenylpropionate (aka hydrocinnamate) were increased in subjects following sumac consumption. Cinnamoylglycine is a glycine conjugate of cinnamic acid, a urinary metabolite in humans (366). Whether it is formed de novo from plant cinnamic acid or is a plant product excreted unchanged has not been conclusively demonstrated. Importantly, it is suggested to be a biomarker for colonization resistance to Clostridium difficle and, therefore, may exert antimicrobial activities in the colonic lumen (360). 3-phenylpropionic acid is a metabolite formed by human colonic microflora from both phenylalanine and proanthocyanidin polymers (356). Benzoic acid is a known derivative of 3-phenylpropionic acid, but the former was not altered in our subjects, suggesting a rapid conversion of benzoic acid into hippurate or 
conversion to alternate glycine-conjugated forms such as phenylpropionylglycine (190, 356, $357)$.

Lastly, several gamma-glutamyl amino acids (GGAA) were decreased in subjects following sumac sorghum consumption. GGAA are produced by gamma-glutamyl transferase (GGT), a cell surface enzyme, during the transfer of gamma-glutamyl functional groups from glutathione to an amino acid acceptor (400). Thus, GGT plays a crucial role in the recycling/resynthesis of glutathione and, to a lesser extent, the transport of amino acids into cells. Importantly, numerous studies have identified elevated GGT levels as a biomarker for metabolic syndrome and cardiovascular disease risk (368-371, 377). Lower levels of gamma-glutamyl amino acids were observed in the serum of subjects after consumption of sumac sorghum, which suggests increased absorption of these molecules from circulation or reduced production by GGT. Previous studies have identified a primary role of GGT in metabolizing extracellular reduced glutathione, which generates gamma-glutamyl amino acids that are then transported into the cell, where the amino acids are liberated to be reused in the synthesis of glutathione (401). Glutathione is responsible for preventing damage to the cell's components produced by various reactive oxygen species such as lipid peroxides, peroxides, free radicals, and heavy metals (402). Thus, lower levels of gamma-glutamyl amino acids in the blood could reflect lower levels of reduced glutathione (i.e., lower oxidant stress), that could be compensated by antioxidant activities afforded by sumac sorghum derived metabolites in circulation. Additionally, researchers have proposed that gamma-glutamyl amino acids could be used as a biomarker for colonization resistance by bacteria from the Lachnospiraceae and Ruminococcaceae families (255). In our experiment, bacteria belonging to the genus Lachnospiraceae incertae sedis exhibited the greatest reduction of all genera in response to sumac sorghum consumption. 
Following the results of our plasma metabolomics profiling, which revealed several microbially-derived compounds increased in response to sumac sorghum consumption, we hypothesized that intermediary metabolites which are known or highly suspected to be derived from the microbiota would be increased in fecal samples collected at the same time point. Additionally, our fecal microbe profiling had demonstrated shifts in the microbiota in response to sumac sorghum consumption, which could later be correlated with plasma and fecal metabolites to identify potential contributors to the metabolic shifts detected in the feces and in circulation. Out of the 8 metabolites chosen for measurement (based on known literature, standard availability, and validation), only four were detected and quantifiable in enough subjects to conduct statistical analyses. Potential explanations for undetected metabolites include, 1) the chemical standard validated by the method is not a form of the metabolite present in vivo, 2) that the levels of target metabolites are truly below the level of detection and/or quantification, and 3) that the extraction procedures employed were not sufficient and/or appropriate to recover the metabolites of interest. 3-(3-hydroxyphenol)propionic acid, 3-phenylpropionic acid, and benzoic acid, all derivatives of proanthocyanidin polymers, were increased in subjects following sumac sorghum consumption; however, not all subjects had detectable/quantifiable levels of these compounds post-consumption. This inconsistency could be due to lack of microbial catabolic function necessary to generate these derivatives in all subjects or differences in production, absorption, and/or conversion rates. Variability in subject microbial profiles clearly indicate this as a possible contributor. Furthermore, although we do not believe that compliance was an issue according to the diet records, subjects did not consume the dietary intervention under direct supervision by investigative staff and, therefore, must be considered. Nevertheless, the increase in concentration of these metabolites in the fecal stream as a result of sumac sorghum 
consumption makes them ideal candidates for future in vivo or in vitro mechanistic studies. More work will be necessary to determine to what extent these metabolites can act locally on host physiology at the site of production (i.e., the intestinal epithelium), how they can impact the microbiota composition, and how they can impact other risk factors related to metabolic syndrome. Quinic acid was reduced in subjects following sumac sorghum, despite it being a microbial derivative of chlorogenic acid, found in sorghum, and a precursor of benzoic acid $(179,209)$. Furthermore, quinic acid is a major precursor of hippuric acid $(354,355)$.

Taken together, our results suggest that a modest intake of sumac sorghum can induce putatively beneficial shifts in gut microbiota and microbially-derived metabolites in the intestinal lumen and in circulation. Much of the literature linking specific gut microbes and their metabolites to positive health outcomes are based on correlative analyses in diseased and healthy, or lean and obese subjects or rodent models. It will be necessary to advance the work presented in this study and others by conducting highly controlled, mechanistic studies to elucidate causal bioactive functions of these microbes and their metabolites. Significant variability in nearly all parameters measured prevents our ability to detect smaller and potentially relevant changes with our sample size. Statistically, it is incredibly, perhaps impossibly, difficult to control for every factor (e.g., genetics, age, diet, etc.) that might contribute to the composition and functional repertoire of the gut microbiome. As diet and exercise are significant modifiers of the microbiome, future studies would benefit from stricter inclusion and exclusion criteria and should seek to control the activity and diet of subjects as much as possible (e.g., exercise regimes, and preparation, provision, and supervision of all meals/experimental interventions for better control and compliance). 
Some bacteria have been well described in terms of their metabolic activity and contribution to host health (e.g., H. pylori, E. coli, etc.); however, the majority of microbiome constituents remain to be fully characterized. This lack of information precludes our ability to associate the production of the detected metabolites with a given a bacterium. Targeted fecal metabolite profiling revealed significant increases in metabolites derived from dietary proanthocyanidins contained in sumac sorghum, confirming their presence in the fecal stream. These compounds may act locally on the intestinal tissue and systemically before undergoing further metabolism by host enzymes, such as conjugation in intestinal and hepatic cells. Although we have not yet confirmed the protein levels of GGT, detection of lower levels of gamma-glutamyl amino acid metabolites suggests sumac sorghum in the diet may have the potential to modulate GGT activity or the availability of its substrate, which may serve to mitigate oxidative stress while simultaneously reducing the burden on intrinsic antioxidant networks in the host.

Phenotypes associated with the risk of metabolic disease are strongly correlated with unhealthy (i.e., high fat, low fiber, etc.) dietary consumption patterns. Concurrent with this observation are functional and compositional changes in the gut microbiota that have the potential to promote disease development. Moreover, the functional capabilities of the microbiota can affect the nutritional value to the host of a given food. Thus, there is a complex, trilateral interaction that exists between the host, diet, and microbiota, whereby the diet can directly, via host digestive processes, and indirectly, via microbial digestion, affect host metabolism. For this reason, research characterizing the functional potential of a given microbiome will be necessary to elucidate the mechanisms that define host-microbial symbiosis. Dietary studies correlating metagenomic and metabolomic analyses will also provide a basis for 
describing an individual's microbial fingerprint and, thus, their susceptibility to pre- and probiotic intervention. 


\section{CHAPTER V}

\section{RELATING THE MICROBIOME AND THE METABOLOME: A SECONDARY ANALYSIS OF THE KANSAS SORGHUM STUDY DATA}

\section{Introduction}

Sequencing of the $16 \mathrm{~S}$ microbial gene permits us to determine whether the incorporation of a polyphenol-rich sorghum cereal into the diet had any effect on the composition of the microbiota but gives no indication of whether these changes have any effect on the physiology of the host. As many microbial metabolites are known to be absorbed and utilized/metabolized by the host, plasma metabolomics allows the identification and relative quantification of dietinduced, microbially-derived compounds that are in circulation. Unfortunately, the physiological processes underpinning these observations are highly uncharacterized and further complicated by microbial gene redundancy, which allows many different bacteria to perform the same or similar functions. For example, there are many species of bacteria known to synthesize butyrate. Therefore, our goal was to determine relationships between metabolomic and microbial variables, so that future experiments could focus on identifying which bacteria may be responsible for the generation of specific metabolites that are absorbed by the host. The analysis variables of interest were: 1) dose response (i.e., low vs. high change from baseline); 2) mean change across diets (i.e., treatment response); 3) high diet change from baseline; and 4) low diet change from baseline. 


\section{Methods and materials}

$\underline{\text { Study design and dietary intervention }}$

The procedures used in this study were approved by the Institutional Review Boards of Texas A\&M University, Kansas State University and University of Nebraska and written informed consent was obtained from all subjects $(n=24)$ who participated. Males and females between the ages of 25-60 years, who were: nonsmokers; not taking medications for weight loss; not currently dieting for weight loss (weight had not changed more than $5 \mathrm{~kg}$ in either direction during the last 3 mo); and had a BMI between 26 and 29 were recruited. Subjects must have been able to read, write and understand the English language. Subjects were excluded if they: did not regularly eat breakfast or snacks; had food allergies; had cardiovascular disease; were diabetic; had cancer in the 5 years prior to the study (except basal cell skin carcinoma); had renal or hepatic disease; had weight loss or gain of more than $5 \mathrm{~kg}$ in the previous 3 months (intentional or unintentional); had a history of drug or alcohol abuse in the previous 6 months; were taking weight loss, lipid-lowering, anti-hypertensive or anti-inflammatory steroid medications; had Crohn's disease, ulcerative colitis or other digestive disorders that interfere with digestion or absorption; women who were pregnant or lactating; women who did not have a regular menstrual cycle; were vegetarians; or people that consumed more than 15 grams of fiber per day.

The study employed a randomized crossover design with two levels of sumac sorghum dietary intervention: low, $50 \mathrm{~g} /$ day and high, $100 \mathrm{~g} / \mathrm{day}$. The sumac sorghum intervention was an extruded cereal puff containing only $95 \%$ sumac flour and $5 \%$ sumac bran that resembled a cocoa puff in texture, color, and bowl life (i.e., how long the cereal retained texture when milk was added). Subjects were provided with an optional teaspoon sugar sachet to use if desired. A TX52 twin screw extruder (Wenger Mfg., Sabetha, KS) was used to process the extruded puffs. 
Subjects consumed each level of intervention for 4 weeks, separated by a 3-week washout period. Subjects were instructed not to change exercise regimen or diet, aside from the intervention. Samples were collected at baseline and washout time points and following each intervention period. Samples for metabolite analysis were collected in the morning following a 10-hour overnight fast. Details of the methods and materials employed for the generation of microbial and metabolite data are presented in Chapter IV.

$\underline{\text { Statistical methods }}$

The following analyses were carried out separately for each level of sumac sorghum consumption (i.e., Low, 50 g/day and High, 100 g/day).

Inclusion criteria and summary statistics for microbial and metabolite data analysis

Due to the variability observed in the microbial and metabolomic data (i.e., not all subject samples contained all detected microbes or metabolites) inclusion criteria were established for the data prior to statistical analysis. The inclusion criteria for the analyses were as follows: 1) subjects with complete data were included (one subject with microbial measurements but no metabolite data was excluded), 2) a microbe was included if at least 12 subjects had at least two non-zero values of the four measurements (pre and post for the two diets, low and high), referred to as the $50 \%$ version rule, and 3) a metabolite was analyzed if there were no missing values (i.e., $\mathrm{N}=23$ subjects).

Due to a high dimensional data set (i.e., number of variables), principal component (PCA) and canonical correlation (CCA) analyses were performed to attain lower dimensional data that were analyzed by metabolite and microbial pair using Pearson correlation (403). Statistical conclusions are based on the Pearson correlation results adjusted for multiple comparisons using the FDR method by taxon and subpathway, with $\alpha=0.05$. 
PCA is a multivariate method used for high-dimensional data reduction that permits the extraction of variables which are most important in a highly dimensional data table $(404,405)$. Original variables were standardized and transformed into a set of orthogonal principal components with corresponding eigenvalues and eigenvectors. Principal component eigenvalues are ordered from highest to lowest proportion of variance explained. PCA was performed separately by taxon for microbial variables and by subpathway for metabolite variables. Microbiota were included using the 50\% rule for zeros (at least 12 subjects have at least two non-zero values of the four measurements). All biochemicals with non-missing data were included in the analysis. The selection criteria for evaluation of a principal component was: 1) cumulative variance $<95 \%$; and 2) $>5 \%$ variance explained. For each evaluated principal component, a variable was included if the absolute value of its coefficient was $>0.45$. For principal component analysis of microbial taxon genus with $n=32$ (greater than subject $n=23$ ), 300 random samples of 22 were analyzed and selection of microbiota was based on frequency percent.

The selected microbiota and metabolites from PCA were analyzed using canonical correlation by taxon and subpathway ( $8 \times 54$ analyses). CCA is a multivariate method used to analyze relationships between two sets of random variables by optimizing the cross-covariance (406). Similar approaches have been used to identify potential relationships between microbes and metagenomic gene signatures (407). A canonical variate pair consists of a linear combination of each set of variables with maximized correlation between the linear combinations. The number of pairs equals the number of variables in each dataset (or in the smaller dataset if unequal). The canonical correlation for each canonical pair is tested by row using an $\mathrm{F}$ test for the null hypothesis (i.e., $\mathrm{H}_{0}$ : The canonical correlation in the current row and all that follow are 
zero). The inclusion criteria was $P$-value $<0.10$. For each canonical pair selected, the correlations of the canonical variates (biochemical and microbial) with each variable were evaluated. The correlation of a microbial or biochemical variable with its own canonical variate or opposite canonical variate was absolute value $>0.40$ for inclusion.

For the microbiota and metabolites selected using canonical correlation analysis, Pearson correlation was performed for the statistical results and conclusions. Analysis was conducted for all microbial and metabolomics pairs by taxon and subpathway with FDR adjustment for multiple comparisons.

\section{Results}

Pearson correlation of $\Delta$ Low and $\Delta$ High microbiota (i.e., genus level) and plasma metabolite data are presented in Tables 22 and 23, respectively. A number of significant correlations were identified after dimensional reduction and false discovery rate correction of $P$ values. For the low level of consumption, a positive association with Bacteroides was seen for the gamma-glutamyl amino acids gamma-glutamylvaline $(r=0.582, Q=0.008)$ and gammaglutamylleucine ( $r=0.537, Q=0.012$ ). Gamma-glutamylvaline was negatively associated with Streptococcus ( $r=-0.441, Q=0.036)$ and Faecalibacterium $(r=-0.465, Q=0.032)$, and the latter with Faecalibacterium ( $r=-0.577, Q=0.008$ ). Glutamate, an important neurotransmitter and necessary molecule for the synthesis of glutathione, was positively associated with Dialister ( $r=0.522, Q=0.025)$, and negatively associated with Blautia $(r=-0.411, Q=0.046)$ and Faecalibacterium ( $r=-0.481, Q=0.025)$. 3-hydroxybutyrate was positively associated with Dialister ( $r=0.508, Q=0.020)$ and Akkermansia $(r=0.433, Q=0.034)$ genera and negatively associated with Blautia ( $r=-0.489, Q=0.020)$ and Streptococcus $(r=-0.513, Q=0.020)$. 
Table 22. Significant correlations between plasma metabolites and microbes (genus level) in response to the low level (50 g/day) consumption of dietary sumac sorghum in human subjects.

\begin{tabular}{|c|c|c|c|c|c|c|c|}
\hline \multicolumn{3}{|c|}{ Low consumption (50 g/day) } & \multicolumn{5}{|c|}{$\begin{array}{c}\text { Pearson Correlation } \\
\end{array}$} \\
\hline Subpathway & Biochemical & Genus & Est. $(r)$ & Lower CL & Upper CL & $P$-Value & $Q$-value \\
\hline Chemical & 2-ethylhexanoate & Bacteroides & 0.614 & 0.271 & 0.819 & 0.001 & 0.002 \\
\hline Gamma-glutamyl & gamma-glutamylvaline & Bacteroides & 0.582 & 0.224 & 0.802 & 0.002 & 0.008 \\
\hline Carnitine metabolism & decanoylcarnitine & Blautia & 0.573 & 0.211 & 0.797 & 0.003 & 0.008 \\
\hline Gamma-glutamyl & gamma-glutamylleucine & Bacteroides & 0.537 & 0.160 & 0.777 & 0.006 & 0.012 \\
\hline Glutamate metabolism & glutamate & Dialister & 0.522 & 0.140 & 0.769 & 0.008 & 0.025 \\
\hline Ketone bodies & 3-hydroxybutyrate & Dialister & 0.508 & 0.122 & 0.761 & 0.010 & 0.020 \\
\hline Sugar, sugar substitute, starch & erythritol & Akkermansia & 0.502 & 0.113 & 0.757 & 0.012 & 0.024 \\
\hline Carnitine metabolism & cis-4-decenoyl carnitine & Blautia & 0.488 & 0.095 & 0.750 & 0.015 & 0.015 \\
\hline Fatty acid metabolism & propionylcarnitine & Blautia & 0.470 & 0.072 & 0.739 & 0.020 & 0.049 \\
\hline Ketone bodies & 3-hydroxybutyrate & Akkermansia & 0.433 & 0.026 & 0.717 & 0.034 & 0.034 \\
\hline Glutamate metabolism & glutamate & Blautia & -0.411 & -0.704 & 0.001 & 0.046 & 0.046 \\
\hline Sugar, sugar substitute, starch & erythritol & Bacteroides & -0.423 & -0.711 & -0.013 & 0.039 & 0.039 \\
\hline Gamma-glutamyl & gamma-glutamylvaline & Streptococcus & -0.441 & -0.722 & -0.036 & 0.030 & 0.036 \\
\hline Chemical & iminodiacetate & Bacteroides & -0.446 & -0.725 & -0.042 & 0.028 & 0.028 \\
\hline Fatty acid metabolism & propionylcarnitine & Dialister & -0.457 & -0.731 & -0.055 & 0.024 & 0.049 \\
\hline Gamma-glutamyl & gamma-glutamylvaline & Faecalibacterium & -0.465 & -0.736 & -0.066 & 0.021 & 0.032 \\
\hline Glutamate metabolism & glutamate & Faecalibacterium & -0.481 & -0.745 & -0.085 & 0.017 & 0.025 \\
\hline Ketone bodies & 3-hydroxybutyrate & Blautia & -0.489 & -0.750 & -0.096 & 0.015 & 0.020 \\
\hline Ketone bodies & 3-hydroxybutyrate & Streptococcus & -0.513 & -0.764 & -0.128 & 0.010 & 0.020 \\
\hline Carnitine metabolism & decanoylcarnitine & Dialister & -0.514 & -0.764 & -0.129 & 0.010 & 0.013 \\
\hline Krebs cycle & citrate & Bacteroides & -0.518 & -0.767 & -0.135 & 0.009 & 0.035 \\
\hline Cys, Met, SAM, Tau metabolism & 2-hydroxybutyrate & Streptococcus & -0.526 & -0.771 & -0.146 & 0.008 & 0.008 \\
\hline Carnitine metabolism & cis-4-decenoyl carnitine & Dialister & -0.556 & -0.787 & -0.186 & 0.004 & 0.008 \\
\hline Long chain fatty acid & palmitate $(16: 0)$ & Ruminococcus & -0.557 & -0.788 & -0.188 & 0.004 & 0.004 \\
\hline Gamma-glutamyl & gamma-glutamylleucine & Faecalibacterium & -0.577 & -0.799 & -0.216 & 0.003 & 0.008 \\
\hline
\end{tabular}


Table 23. Significant correlations between plasma metabolites and microbes (genus level) in response to the high level (100 g/day) consumption of dietary sumac sorghum in human subjects.

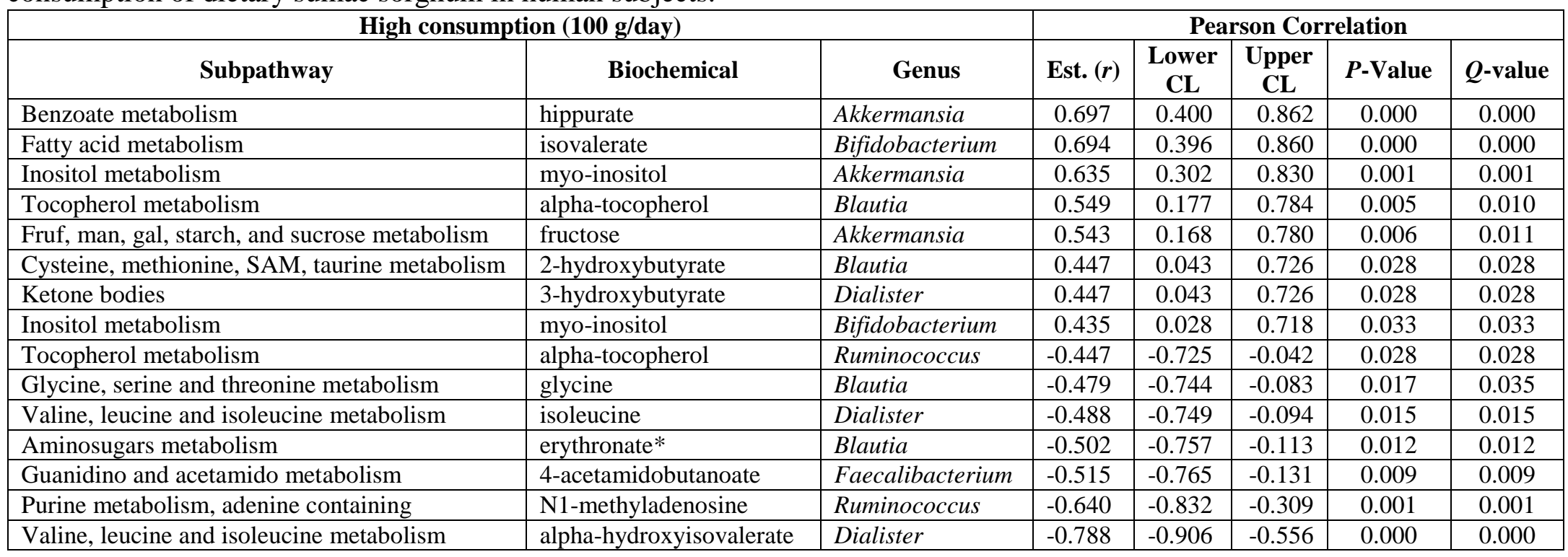


Interestingly, correlation analysis of data sets for the high level of sumac sorghum consumption, revealed, for the most part, relationships for different metabolites, than was observed for the low level of consumption. No associations were seen for gamma-glutamyl amino acids and glutamate; however, 3-hydroxybutyrate remained positively correlated with Dialister bacteria ( $r=0.447, Q=0.028$ ). Most intriguingly, the microbially-associated metabolite hippurate was positively correlated with the presence of Akkermansia ( $r=0.697, Q=0.000$ ). Akkermansia was also found to correlate positively with myo-inositol ( $r=0.635, Q=0.001$ ), which was also positively associated with Bifidobacterium ( $r=0.435, Q=0.033)$. Isovalerate, a branched chain isomer of the SCFA valerate, was positively correlated with the presence of Bifidobacterium ( $r=0.694, Q=0.000)$.

\section{Discussion}

The gut microbiome and its metabolism is highly integrated with the physiology of the host and novel associations of microbial contribution on multiple host systems is being discovered by research at an accelerating pace (408-411). The ability of the intestinal microbiome to metabolize dietary compounds, along with substrate produced by the host (e.g., bile acids, mucins, etc.), into metabolites that can affect host tissues is a fascinating and complex system that will take many years to fully decrypt. Just as host organs work in symphony to generate energy, circulate metabolites, and detoxify waste and unwanted biochemicals, so too does the microbiome. Increasingly, the microbiome is referred to as a host 'organ system' (412417). However, unlike other mammalian organ systems which may exhibit alterations in gene/protein/epigenetic profiles, changes in composition of the microbiome can result in alterations to the metagenome. This 'plasticity' of the microbiome, especially in free living human subjects, precludes our ability to ascribe changes in a given host parameter to individual 
bacteria. Furthermore, given the extent of interactions possible between different bacterial species, it is more likely that the collective profile of the microbiome contributes to the phenotypic response observed at the time of scientific investigation. The exceptions are bacterium which have been abundantly characterized and rigorously investigated for their functions in vivo (e.g., E. coli and H. pylori) (418-422). Furthermore, it has been suggested that the functional profile (i.e., metagenome, metatranscriptome, and metaproteome) of the microbiota is better for classifying microbial communities, particularly in the context of disease and metabolism (215). For example, although certain bacterial species are known to be producers of SCFA, the abundance of critical genes involved in SCFA production, their expression, and translation would provide a much better characterization of the metabolic activity for a given microbiome sample.

The sumac sorghum intervention contains dietary polyphenols which are known to impact, and undergo metabolism by, bacteria in the large intestine. Unfortunately, due to the extremely large amount of variables (i.e., high dimensionality) in each data set (i.e., microbe and metabolite), dimensional reduction analyses and inclusion criteria were imposed on the raw data in order to make the correlation analyses more manageable. The trade-off with employing such procedures is that not all variables are utilized in the final analyses. For example, some of the plasma metabolites that were significantly increased in subjects following consumption of the sumac sorghum were not included in the analysis due to their low proportion of variance as revealed by principal component analysis, or due to missing data. However, these compounds are of particular interest due to their identification as microbially- or polyphenol-derived compounds. It will, therefore, be necessary to revisit these data in the future with more specific 
analyses aimed at identifying and including metabolites and bacteria with known or suspected association.

Gamma-glutamyl amino acids are the byproduct of gamma-glutamyl transferase (GGT), an enzyme involved in the synthesis and degradation of glutathione and drug and xenobiotic degradation (423). Elevated GGT is a biomarker for cardiovascular disease risk (368, 370, 371, 377). Lower levels of gamma-glutamyl amino acids were observed in the serum of subjects after consumption of sumac sorghum, which suggests increased absorption of these molecules from circulation or reduced production by GGT. Previous studies have identified a primary role of GGT in metabolizing extracellular reduced glutathione, which generates gamma-glutamyl amino acids that are then transported into the cell, where the amino acids are liberated to be reused in the synthesis of glutathione (401). Glutathione is responsible for preventing damage to the cell's components produced by various reactive oxygen species such as lipid peroxides, peroxides, free radicals, and heavy metals (402). Thus, lower levels of gamma-glutamyl amino acids in the blood could reflect lower levels of reduced glutathione (i.e., lower oxidant stress), that could be compensated by antioxidant activities afforded by sumac sorghum derived metabolites in circulation. The results of our correlative work suggest that an increased abundance of Bacteroides genera in the gut is associated with increased gamma-glutamyl amino acid levels in the blood, and therefore the possibility of elevated oxidative stress. Conversely, a negative relationship between Faecalibacterium, and perhaps to a similar extent Streptococcus, and gamma glutamyl-amino acid levels suggests that these bacteria could be associated with decreased oxidative stress. Importantly, Faecalibacterium has been described as an antiinflammatory, commensal bacterium that is negatively associated with colitis, and positively associated with maintenance of clinical remission in ulcerative colitis (386-389). Butyrate- 
dependent $(390,391)$ and -independent $(387,388)$ mechanisms have been proposed to explain the role of Faecalibacterium in colitis and in the promotion of intestinal barrier function and inflammation suppression. Although glutamate is involved in the synthesis of glutathione, a relationship linking circulating levels of glutamate and the microbiome has not been fully described. There are species of anaerobic bacteria that are known to ferment glutamate to produce acetate, butyrate, carbon dioxide, and ammonia (424) and others that are known to produce it (425); however, none of these species were implicated by our analyses. In our subjects, plasma glutamate levels were decreased following sumac sorghum consumption and its precursor molecule, glutamine, was decreased (data not shown).

3-hydroxybutyrate levels were not significantly affected by sumac sorghum consumption, but were correlated with various genera in the low and high consumption data sets. 3hydroxybutyrate (aka $\beta$-hydroxybutyrate, BHB), a ketone body and histone deactylase inhibitor (HDAC), has been shown to increase in various tissues including the brain following exercise, calorie restriction, fasting, and ketogenic diets, and is thought to promote improvements in cognition, depression and anxiety via increases in brain derived neurotropic factor (BDNF) (426). BHB is synthesized in the liver via metabolism of fatty acids such as butyrate, a histone deacetylase inhibitor (HDAC) $(427,428)$. Butyrate, a SCFA produced by microbial fermentation of dietary fiber in the gut, is a highly regarded molecule for its role in intestinal health, metabolism, and immunity $(28,77,148,156,158,332,429,430)$. Recently, potential synergies of BHB and butyrate on the modulation of metabolism, inflammation, cognition, and overall health have been reviewed (428). Dialister, which was positively associated with plasma BHB levels in our experiment, was increased in humans consuming $60 \mathrm{~g}$ of whole-grain barley and correlated with reduced plasma IL-6 levels, implicating them as functionally relevant in the 
physiologic effects of whole grains (350). Furthermore, Akkermansia, which was also positively associated with BHB levels, has been shown to improve negative metabolic outcomes of a high fat diet in mice, including: weight gain, epithelial integrity, and lipopolysaccharide endotoxemia (431). In clinical studies, negative associations between metabolic disorders and Akkermansia have been observed; however, more work is needed to clarify contrasting observations in some studies (304). Nevertheless, our correlative work suggests a potential relationship between these species and systemic levels of BHB.

Interestingly, many of the significant correlations that were observed for the low level (50 g/day) of sumac sorghum consumption were not observed in our analyses of the high level (100 g/day) of sumac sorghum consumption. This is possibly a dose-response effect of the dietary intervention affecting which metabolites and microbes contribute the greatest variability in the data set, and are therefore selected for correlation analysis by PCA. Furthermore, as these data were obtained from samples taken at different time points, and our subjects were free living humans, it is likely that other unaccounted factors may have contributed.

Most intriguingly in the high sumac data analysis, is the positive association between hippurate and Akkermansia. Hippurate is the glycine conjugate of benzoic acid, and was increased in our subjects' plasma following sumac sorghum consumption. Hippurate has long been associated with the microbial catabolism of dietary phenolic and polyphenolic compounds (432) and is negatively associated with impaired glucose tolerance (376). In fact, germ free animals fed a polyphenolic diet are unable to secrete hippurate or related metabolites, which implicates the microbiota as the source of these compounds $(433,434)$. Furthermore, there is substantial evidence demonstrating that dietary polyphenols from a variety of sources are able to promote the growth and abundance of Akkermansia in the gut $(305,307,309,393)$. Taken 
together, our findings and others' support the identity of Akkermansia as a significant contributor to host health and metabolism, particularly in response to diets high in polyphenolic compounds. For this reason, Akkermansia warrants greater investigation in future studies aimed at elucidating the beneficial effects of dietary polyphenols on host health and metabolism.

In these analyses, we have attempted to identify potential associations between metabolites found in the plasma of human subjects consuming a sumac sorghum dietary intervention and the various microbial genera in fecal samples. Indeed, our data demonstrate that bacteria suggested in the literature to be promotive of intestinal and metabolic health (i.e., Faecalibacterium and Akkermansia) are correlated with metabolites identified as potential contributors to these host responses (i.e., polyphenolic derivatives and gamma-glutamyl amino acids). However, we want to emphasize that the correlations discussed, while significant, are not particularly strong and their interpretation here is merely suggestive of a potential relationship. In other words, these data do not confirm that a change in one variable (microbe or metabolite) definitively causes a change in the other. Nevertheless, these correlative associations provide useful preliminary data for further investigation of physiologically relevant host-microbe interactions. 


\section{CHAPTER VI}

\section{SUMMARY AND CONCLUSIONS}

According to a 2017 published review of cancer statistics (435), colon and rectal cancer is the third most commonly diagnosed cancer type in men and women in the United States and the second and third leading cause of cancer related death, in men and women, respectively, in the United States. Despite significant improvements in screening and treatment and an overall decline in incidence and mortality of colorectal cancer in individuals over the age of 50, incidence and death rates have continued to increase (22\% and 34\%, respectively) in individuals under the age of 50 and is driven solely by cancers of the distal colon and rectum (239). This fact has influenced the American Cancer Society (cancer.org) to update its guidelines for colorectal cancer screening to begin at age 45 for average risk individuals. For the scientific community, this has brought significant attention to the biological differences in cancer between that of the distal colon/rectum and the proximal colon and understanding the processes that contribute to the unique microenvironments that exist throughout the large intestine. Most notable are differences in the gene expression, epigenetic profile, and molecular signature of the tumor, as well as the composition of the microbiome at the disease site.

Microbiota are capable of significantly impacting host physiology at a localized and systemic level, and additional interactions between microbe activity and host processes are being uncovered by research at an astonishing rate. The magnitude of host-microbe interaction and ability for diet and other factors to impact the composition and metabolism of the microbiome further supports the classification of the microbiome as an organ system, which may arguably prove to be the most difficult to fully comprehend and characterize. In vivo studies employing 
dietary interventions in the context of disease and analyzed with 'omics-style profiling stimulate hypotheses for targeted, mechanistic basic research. The experiments presented in this dissertation have demonstrated the ability of foods containing bioactive polyphenols to impact the host and gut microbiome, with implications for disease prevention and treatment. Dietary bioactives (e.g., polyphenols) are extra-nutritional components of fruits, vegetables, and grains with the ability to impact colitis, cancer, bone loss, cardiovascular risk, and diabetes (436). These effects are thought to be due in large part to their anti-oxidant and anti-inflammatory properties, but research has demonstrated that the bioactivity and bioavailability is largely dependent on the capacity of the microbiome to metabolize and generate secondary metabolites which may be responsible for the observed effects in vivo. Alterations in gut microbe composition, increases in microbially-derived metabolites in feces and circulation, and absence of the latter in germ free (aka gnotobiotic) animal models following polyphenol consumption strongly implicate the microbiome as a significant contributor to the host response to diet. A greater understanding of the extent and capacity of the diet-host-microbe trilateral relationship will ultimately revolutionize medicine as the world enters the era of personalized, genome-based healthcare and nutrition.

To our knowledge this is the first study employing a plum based dietary intervention at a level directly translatable to the recommended consumption of dried plums for humans (i.e., a 40 g serving providing $5 \%$ of calories in a standard 2000 calorie per day diet). Previous experiments have used individual bioactive compounds found in dried plum (e.g., chlorogenic acid and quercetin) or a dried plum powder to investigate protective mechanisms of dried plum in the context of colon cancer and inflammation $(196,205,437)$. Our results indicate that by feeding dried plum prior to initiation, and throughout early stages of colon carcinogenesis, it is possible 
to reduce the number and multiplicity of preneoplastic lesions and reduce the expression of genes contributing to inflammation. Specifically, dried plum reduced the expression of iNOS throughout the colon, which was increased during carcinogenesis, and decreased the expression of Tlr2 and Tlr4 microbial recognition transcripts in the proximal colon in carcinogen treated animals. The translation of the iNOS transcript into the iNOS protein (inducible nitric oxide synthase) results in the subsequent generation of the potent signaling molecule and free radical immune response effector, nitric oxide (438). Although it is regarded as a host defense against pathogen infection, its lack of specificity can lead to DNA damage and mutation, neovascularization, and nitrosylation of proteins (e.g., p53, caspase, etc.) which can promote carcinogenesis (266). TLR signaling following microbial pattern recognition activates a signaling cascade via $\mathrm{NF}-\kappa \mathrm{B}$ which leads to expression of inflammatory cytokine gene, thereby regulating the immune response to infection. Abnormal regulation of $\mathrm{NF}-\kappa \mathrm{B}$ signaling is implicated in colitis and cancer (439). Therefore, there is potential for dried plum to protect against colon carcinogenesis at initiation and promotion stages; however, the design of our experiment precludes our ability to assess the former, but supports the identity of dried plums as a mitigator of the pro-inflammatory immune response characteristic of early carcinogenesis. Part of these effects could be due to alterations in SCFA production or absorption; however, we did not observe a significant effect of dried plum on SCFA transporter expression, which was reduced in the distal colon of carcinogen treated animals. Increases in excreted SCFA following carcinogen treatment in basal diet animals, but not in plum diet animals, suggests dried plum is able to support a steadier pattern of SCFA metabolism following cancer initiation. Dried plum animals had lower levels of several SCFA in feces compared to basal diet animals, and the same trend was observed in the intestinal lumen of mice fed a dried plum diet. Therefore, it is apparent 
that dried plum consumption alters the SCFA profile compared to a basal diet, but also enables the steady-state maintenance of that profile during colon cancer initiation, when SCFA such as butyrate can act early to initiate apoptosis in DNA damaged stem cells (77, 270, 440). This could explain, in part, the reduction in pre-neoplastic lesion incidence and multiplicity observed 8 weeks later at termination.

Dried plum induced substantial alterations in the composition of the microbiota and colonic metabolome, particularly in the distal colon of rats in the first experiment. However, exposure to the chemical carcinogen (Chapter II) or radiation (Chapter III) had minimal impact on the composition of the microbiota. Despite no changes in alpha diversity in either experiment, rats from Chapter II clustered significantly based on diet in estimates of diversity between samples. In mice exposed to radiation and consuming dried plum a significant increase in Enterobacteriaceae and Akkermansia bacteria was observed, but only in $40 \%$ of animals belonging to that treatment group. Taken together, these observations confirm that dried plum significantly impacts the composition of the colon microbiota including dramatic shifts in dominant bacterial groups; however, it is inappropriate to draw any conclusions regarding the mechanism of protection of dried plum consumption on the formation of ACF (Chapter II) solely based on the observed changes in microbe composition. Furthermore, differences in the composition of gut bacteria between the Sprague Dawley rat (Rattus norvegicus, Chapter II) and C57BL/6 (Mus musculus, Chapter III), make comparisons between these experiments difficult as the bacteria that dominate their guts belong to different groups in the lower taxonomy.

Our analysis of the predicted metagenome and fecal metabolome in Chapter II suggests that dried plum consumption results in changes to the functional capacity and metabolism of the microbiome, respectively. These observations substantiate the notion that dietary modification of 
the microbiome can impact the profile and metabolic potential of the gut microbiome as an organ system, with implications for affecting cancer and disease risk. Pathway analysis of the metabolomic changes implicate several pathway nodes implicated in colon cancer, that can be investigated in greater focus in future experiments.

Sorghum is an extremely versatile grain containing a good source of nutrients and bioactive compounds (e.g., 3-deoxyanthocyanins and tannins), and is the fifth most produced cereal in the world (441). Like the bioactive compounds found in dried plums, those in sorghum have been associated with improvements in parameters related to obesity, inflammation, diabetes, and cancer $(179,209,211,442,443)$. Consumption of sorghum results in changes to the composition of the microbiome and microbial-derivatives of sorghum bioactives have been detected in urine, feces, and plasma in both humans and animal models (103, 211, 245, 442, 444). In our experiment, we fed two levels of a bran enriched sumac sorghum puff cereal to human subjects predisposed to metabolic syndrome to see if a low or modest level of sorghum consumption could impact biomarkers related to lipid and glucose metabolism, and inflammation, which are relevant to cardiovascular disease risk, diabetes, obesity, and cancer.

Despite no significant changes in these parameters over the relatively short intervention periods, significant alterations in gut microbe composition and microbially-derived metabolites in feces and plasma were observed. Faecalibacterium was significantly increased and Akkermansia displayed a tendency to increase in subjects after consuming sumac sorghum. Both of these bacteria are associated with beneficial effects on host health. F. prausnitzii, the sole known species of the genus Faecalibacterium, represents more than $5 \%$ of the bacteria in a healthy human intestine $(385,386)$. Prior to beginning the experiment, our subjects averaged 4.5\% of total sequences identified as Faecalibacterium; however, the abundance of these 
sequences increased to $7.2 \%$ following sumac sorghum consumption. F prausnitzii is one of the most abundant butyrate producing bacterium in the gastrointestinal tract, and likely contributes to the anti-inflammatory properties ascribed to $F$. prausnitzii (386). In this experiment, no significant changes were observed in fecal SCFA levels in response to sumac sorghum consumption, although previous work in our lab has indicated that sumac fed animals had the lowest concentration of butyrate compared to Black and Hi Tannin sorghum brans, and cellulose controls (445). Akkermansia is being studied for its impact on human metabolism and has shown protection in against obesity and diabetes (303-305, 307-309, 393, 431). A recent study employing twin cohorts reported that a decrease in Akkermansia was associated with an increased risk of type 2 diabetes and obesity (446). In our experiment, only a trend for increased Akkermansia was observed in response to sumac sorghum consumption (4\% average increase in sequences), and no improvements were documented in fasting blood glucose levels or plasma lipid profiles.

A major limitation of this experiment was the relatively small sample size, and lack of controlled diet and exercise patterns among the subjects. Although subjects were instructed not to change their exercise or dietary regimes aside from the dietary intervention, we can only assume that subjects complied with these restrictions and that they consumed the dietary intervention as prescribed. As subjects were free living, we had no control over the remainder of their diet, which can greatly confound our ability to detect changes because diet is such a potent modifier of the microbiome. Nevertheless, the changes in microbes and microbial-derived metabolites in the plasma and feces, suggest that a longer study and/or one with greater control over subject behavior could yield stronger results. Our final analysis of microbiome and plasma metabolome data implicate both Faecalibacterium and Akkermansia as bacteria which may be 
related in some capacity to the changes observed in the plasma metabolome. Targeted experiments focused on fully characterizing the metabolic profile of these bacteria, perhaps in animal models where the microbiome can be controlled by inoculation of specific bacteria or groups of bacteria, will be necessary to elucidate their unique contributions to the aforementioned observations.

Taken together, these experiments suggest that dried plum and sumac sorghum, both sources of bioactive polyphenols, are modifiers of the microbiome and its metabolism, with important implications for multiple diseases, including cancer, obesity, and diabetes.

\section{Future Studies}

Each experiment demonstrated that modest modifications to the diet, which should be easily achievable for humans to incorporate routinely, can alter the microbiota, mucosal environment, and systemic metabolome. Further studies are warranted to better characterize the mechanisms by which dried plums and sumac sorghum exert their effects on gut and systemic health. In the case of dried plum as a mitigator of azoxymethane-induced colon carcinogenesis, it will be important to determine 1) if dried plum interferes with the ability of the carcinogen to initiate colon carcinogenesis, and 2) if the protection observed at the ACF stage translates into protection at the tumor stage (i.e., reduced tumor size and abundance). The former could easily be tested by introducing the experimental diets after treatment with the chemical carcinogen rather than before. All animals could begin the study consuming a basal diet until the second AOM exposure when then half of the animals would switch to the dried plum diet until termination. The ability of dried plum to prevent the induction of colon carcinogenesis by AOM should not be taken negatively, however, as the mechanisms of its protection in this regard could still be relevant to processes that contribute to spontaneous colon carcinogenesis, which could be 
explored in additional studies. Therefore, it will depend ultimately on the hypothesis being tested by the experiment with careful consideration of the limitations of the model and experimental paradigm. Interestingly, Lactobacillus acidophilus has been shown to reduce ACF formation in AOM treated Sprague Dawley rats (447); however, in our experiment, bacteria of the genus Lactobacillus were reduced in plum diet fed animals, which had lower ACF number and multiplicity.

Although we did not detect many alterations in SCFA or microbe composition in mice exposed to an acute dose of HZE radiation, we know that space relevant doses of HZE can induce shifts in gut microbial populations (104). The most important hurdle of ground-based radiation research for the study of spaceflight, is the difficulty of protracted, low dose-rate exposures that more closely mimic spaceflight, versus acute exposures observed in solar flares, nuclear reactor incidents or nuclear weapon detonations. Therefore, it will be necessary to develop these exposure methodologies to better study the radiation impacts on microbiota and the intestinal environment as they occur over time frames similar to an actual mission duration.

Both dried plum and sumac sorghum require cell culture work to probe for potential mechanisms of bioactivity for the polyphenols contained in them. This work should include both native compounds and those derived from microbial metabolism. It is known that smaller polyphenolic compounds (e.g., monomers and dimers of flavan-3ols) can enter systemic circulation; however, these compounds are often modified by enterocytes/colonocytes during uptake and transport to the liver where they can be further modified (38). Therefore, distinct bioactive profiles are necessary for metabolized and unmetabolized forms of native (i.e., occurring naturally in the foodstuff) bioactive molecules and for their microbial-derivatives, which also undergo host modification following absorption and uptake. It will be important to 
distinguish these bioactivities independently of the presence of the microbiota, which is made possible by the use of germ-free animals. As an alternative to traditional cell culture work, recent advances in cell-microbe co-culture, termed "Gut-On-A-Chip," present an fruitful opportunity to study infinite combinations of cell populations/profiles, substrates, and microbes ex vivo.

Studies employing germ-free animals and animals with discrete microbial populations, in addition to traditional cell culture and co-culture experimentation, will be necessary to dissect the mechanisms and co-dependencies of specific diet-host-microbe interactions. The correlation, or often lack of correlation, between data from stool versus healthy or diseased tissue samples adds enormous complexity to the host-microbe relationship. Therefore, to improve our understanding of these relationships, 'omics' studies should be used to interrogate concomitant changes in the diseased mucosa and the stool to maximize the information gathered from passive fecal sampling. Experiments should use multiple 'omics' approaches to identify those biomarkers that most accurately and robustly characterize the disease state. Furthermore, it will be necessary to assess these relationships throughout the disease process to improve the sensitivity and specificity of diagnostic screening approaches for CRC prevention and treatment.

Future studies attempting to define causal relationships between specific microbiota and disease processes would greatly benefit from more precise sampling techniques, such as laser capture microdissection (LCM). Application of site-specific sampling techniques in combination with more sensitive analytical approaches in future experiments will improve the ability to detect diet-induced modifications of host-microbe interactions in colon cancer models. Targeted quantification of microbial butyrate producing genes, and protein expression of SCFA transporters, would help link our SCFA observations to the microbial populations characterized 
in this study. Furthermore, true metagenome sequencing would give insight on the function of the microbiome and a better characterization of the species present. 


\section{REFERENCES}

1. Seidel DV, Azcarate-Peril MA, Chapkin RS, Turner ND. Shaping functional gut microbiota using dietary bioactives to reduce colon cancer risk. Semin Cancer Biol. 2017 Oct;46:191-204.

2. Ahmed RL, Schmitz KH, Anderson KE, Rosamond WD, Folsom AR. The metabolic syndrome and risk of incident colorectal cancer. Cancer. 2006 Jul 1;107:28-36.

3. Terzic J, Grivennikov S, Karin E, Karin M. Inflammation and colon cancer. Gastroenterology. 2010 Jun;138:2101-14 e5.

4. Datta K, Suman S, Kallakury BV, Fornace AJ, Jr. Heavy ion radiation exposure triggered higher intestinal tumor frequency and greater beta-catenin activation than gamma radiation in APC(Min/+) mice. PLoS One. 2013;8:e59295.

5. Kim JH, Lim YJ, Kim YH, Sung IK, Shim SG, Oh SO, Park SS, Yang S, Son HJ, et al. Is metabolic syndrome a risk factor for colorectal adenoma? Cancer Epidemiol Biomarkers Prev. 2007 Aug;16:1543-6.

6. Kim SB, Bozeman RG, Kaisani A, Kim W, Zhang L, Richardson JA, Wright WE, Shay JW. Radiation promotes colorectal cancer initiation and progression by inducing senescenceassociated inflammatory responses. Oncogene. 2016 Jun 30;35:3365-75.

7. Hanahan D, Weinberg RA. The hallmarks of cancer. Cell. 2000 Jan 7;100:57-70.

8. Block KI, Gyllenhaal C, Lowe L, Amedei A, Amin AR, Amin A, Aquilano K, Arbiser J, Arreola A, Arzumanyan A. Designing a broad-spectrum integrative approach for cancer prevention and treatment. Semin Cancer Biol. 2015;S276-S304.

9. Hanahan D, Weinberg RA. Hallmarks of cancer: the next generation. Cell. 2011 Mar 4;144:646-74.

10. Vander Heiden MG, Cantley LC, Thompson CB. Understanding the Warburg effect: the metabolic requirements of cell proliferation. Science. 2009 May 22;324:1029-33.

11. Feron O. Pyruvate into lactate and back: from the Warburg effect to symbiotic energy fuel exchange in cancer cells. Radiother Oncol. 2009 Sep;92:329-33.

12. Xie J, Wu H, Dai C, Pan Q, Ding Z, Hu D, Ji B, Luo Y, Hu X. Beyond Warburg effect-dual metabolic nature of cancer cells. Sci Rep. 2014 May 13;4:4927.

13. Hammoud SS, Cairns BR, Jones DA. Epigenetic regulation of colon cancer and intestinal stem cells. Curr Opin Cell Biol. 2013 Apr;25:177-83.

14. Pitot HC. The molecular biology of carcinogenesis. Cancer. 1993 Aug 1;72:962-70. 
15. Moody CS, Hassan HM. Mutagenicity of oxygen free radicals. Proc Natl Acad Sci U S A. 1982 May;79:2855-9.

16. Yokota J. Tumor progression and metastasis. Carcinogenesis. 2000 Mar;21:497-503.

17. Lozupone CA, Stombaugh JI, Gordon JI, Jansson JK, Knight R. Diversity, stability and resilience of the human gut microbiota. Nature. 2012 Sep 13;489:220-30.

18. Eckburg PB, Bik EM, Bernstein CN, Purdom E, Dethlefsen L, Sargent M, Gill SR, Nelson KE, Relman DA. Diversity of the human intestinal microbial flora. Science. 2005 Jun 10;308:1635-8.

19. Qin J, Li R, Raes J, Arumugam M, Burgdorf KS, Manichanh C, Nielsen T, Pons N, Levenez $\mathrm{F}$, et al. A human gut microbial gene catalogue established by metagenomic sequencing. Nature. 2010 Mar 4;464:59-65.

20. Kanauchi O, Matsumoto Y, Matsumura M, Fukuoka M, Bamba T. The Beneficial Effects of Microflora, Especially Obligate Anaerobes, and Their Products on the Colonic Environment in Inflammatory Bowel Disease. Curr Pharm Des. 2005;11:1047-53.

21. Marchesi JR, Dutilh BE, Hall N, Peters WH, Roelofs R, Boleij A, Tjalsma H. Towards the human colorectal cancer microbiome. PLoS One. 2011;6:e20447.

22. Qin J, Li Y, Cai Z, Li S, Zhu J, Zhang F, Liang S, Zhang W, Guan Y, et al. A metagenome-wide association study of gut microbiota in type 2 diabetes. Nature. 2012 Oct 4;490:55-60.

23. Sobhani I, Tap J, Roudot-Thoraval F, Roperch JP, Letulle S, Langella P, Corthier G, Tran Van Nhieu J, Furet JP. Microbial dysbiosis in colorectal cancer (CRC) patients. PLoS One. 2011 Jan 27;6:e16393.

24. Tamboli CP, Neut C, Desreumaux P, Colombel JF. Dysbiosis in inflammatory bowel disease. Gut. 2004 Jan;53:1-4.

25. Turner ND, Ritchie LE, Bresalier RS, Chapkin RS. The microbiome and colorectal neoplasia: environmental modifiers of dysbiosis. Curr Gastroenterol Rep. 2013 Sep;15:346.

26. Irrazábal T, Belcheva A, Girardin SE, Martin A, Philpott DJ. The multifaceted role of the intestinal microbiota in colon cancer. Mol Cell. 2014;54:309-20.

27. Reuter S, Gupta SC, Chaturvedi MM, Aggarwal BB. Oxidative stress, inflammation, and cancer: how are they linked? Free Radic Biol Med. 2010 Dec 1;49:1603-16.

28. Greer JB, O'Keefe SJ. Microbial induction of immunity, inflammation, and cancer. Front Physiol. 2011 Dec 23;1:168. 
29. Karin M, Lawrence T, Nizet V. Innate immunity gone awry: linking microbial infections to chronic inflammation and cancer. Cell. 2006 Feb 24;124:823-35.

30. Grivennikov SI, Greten FR, Karin M. Immunity, inflammation, and cancer. Cell. 2010 Mar 19;140:883-99.

31. O'Keefe SJ. Diet, microorganisms and their metabolites, and colon cancer. Nat Rev Gastroenterol Hepatol. 2016 Dec;13:691-706.

32. Salonen A, de Vos WM. Impact of diet on human intestinal microbiota and health. Annu Rev Food Sci Technol. 2014;5:239-62.

33. Riboli E, Norat T. Epidemiologic evidence of the protective effect of fruit and vegetables on cancer risk. Am J Clin Nutr. 2003 Sep;78:559S-69S.

34. Terry P, Giovannucci E, Michels KB, Bergkvist L, Hansen H, Holmberg L, Wolk A. Fruit, vegetables, dietary fiber, and risk of colorectal cancer. J Natl Cancer Inst. 2001 Apr 4;93:525-33.

35. Trock B, Lanza E, Greenwald P. Dietary fiber, vegetables, and colon cancer: critical review and meta-analyses of the epidemiologic evidence. J Natl Cancer Inst. 1990 Apr $18 ; 82: 650-61$.

36. Kris-Etherton PM, Hecker KD, Bonanome A, Coval SM, Binkoski AE, Hilpert KF, Griel AE, Etherton TD. Bioactive compounds in foods: their role in the prevention of cardiovascular disease and cancer. The American Journal of Medicine. 2002 Dec 30;113:71-88.

37. Papandreou D, Noor ZT, Rashed M. The role of soluble, insoluble fibers and their bioactive compounds in cancer: a mini review. Food and Nutrition Sciences. 2015;6:1.

38. Monagas M, Urpi-Sarda M, Sanchez-Patan F, Llorach R, Garrido I, Gomez-Cordoves C, Andres-Lacueva C, Bartolome B. Insights into the metabolism and microbial biotransformation of dietary flavan-3-ols and the bioactivity of their metabolites. Food Funct. 2010 Dec;1:233-53.

39. Aura AM, Martin-Lopez P, O'Leary KA, Williamson G, Oksman-Caldentey KM, Poutanen K, Santos-Buelga C. In vitro metabolism of anthocyanins by human gut microflora. Eur J Nutr. 2005 Mar;44:133-42.

40. Aura A-M. Microbial metabolism of dietary phenolic compounds in the colon. Phytochemistry Reviews. 2008;7:407-29.

41. Clavel T, Henderson G, Alpert CA, Philippe C, Rigottier-Gois L, Dore J, Blaut M. Intestinal bacterial communities that produce active estrogen-like compounds enterodiol and enterolactone in humans. Appl Environ Microbiol. 2005 Oct;71:6077-85. 
42. Barker N, van Es JH, Kuipers J, Kujala P, van den Born M, Cozijnsen M, Haegebarth A, Korving J, Begthel $\mathrm{H}$, et al. Identification of stem cells in small intestine and colon by marker gene Lgr5. Nature. 2007 Oct 25;449:1003-7.

43. Barker N, Ridgway RA, van Es JH, van de Wetering M, Begthel H, van den Born M, Danenberg E, Clarke AR, Sansom OJ, Clevers H. Crypt stem cells as the cells-of-origin of intestinal cancer. Nature. 2009 Jan 29;457:608-11.

44. Potten CS, Wilson JW, Booth C. Regulation and significance of apoptosis in the stem cells of the gastrointestinal epithelium. Stem Cells. 1997;15:82-93.

45. Ruthig DJ, Meckling-Gill KA. Both (n-3) and (n-6) fatty acids stimulate wound healing in the rat intestinal epithelial cell line, IEC-6. J Nutr. 1999 Oct;129:1791-8.

46. Clevers H. The cancer stem cell: premises, promises and challenges. Nat Med. 2011 Mar;17:313-9.

47. Blokzijl F, de Ligt J, Jager M, Sasselli V, Roerink S, Sasaki N, Huch M, Boymans S, Kuijk E, et al. Tissue-specific mutation accumulation in human adult stem cells during life. Nature. 2016 Oct 13;538:260-4.

48. Huels DJ, Sansom OJ. Stem vs non-stem cell origin of colorectal cancer. Br J Cancer. 2015 Jun 30;113:1-5.

49. Bienz M, Clevers H. Linking colorectal cancer to Wnt signaling. Cell. 2000 Oct $13 ; 103: 311-20$.

50. Suraweera N, Robinson J, Volikos E, Guenther T, Talbot I, Tomlinson I, Silver A. Mutations within Wnt pathway genes in sporadic colorectal cancers and cell lines. Int J Cancer. 2006 Oct 15;119:1837-42.

51. Polakis P. Wnt signaling in cancer. Cold Spring Harb Perspect Biol. 2012 May 1;4:a008052.

52. Rycaj K, Tang DG. Cell-of-Origin of Cancer versus Cancer Stem Cells: Assays and Interpretations. Cancer Res. 2015 Oct 1;75:4003-11.

53. Davidson LA, Callaway ES, Kim E, Weeks BR, Fan YY, Allred CD, Chapkin RS. Targeted Deletion of p53 in Lgr5-Expressing Intestinal Stem Cells Promotes Colon Tumorigenesis in a Preclinical Model of Colitis-Associated Cancer. Cancer Res. 2015 Dec $15 ; 75: 5392-7$.

54. Chaffer CL, Weinberg RA. How does multistep tumorigenesis really proceed? Cancer Discov. 2015 Jan;5:22-4.

55. Visvader JE, Clevers H. Tissue-specific designs of stem cell hierarchies. Nat Cell Biol. 2016 Apr;18:349-55. 
56. Wu S, Powers S, Zhu W, Hannun YA. Substantial contribution of extrinsic risk factors to cancer development. Nature. 2016 Jan 7;529:43-7.

57. Tomasetti C, Vogelstein B. Cancer etiology. Variation in cancer risk among tissues can be explained by the number of stem cell divisions. Science. 2015 Jan 2;347:78-81.

58. Tetteh PW, Basak O, Farin HF, Wiebrands K, Kretzschmar K, Begthel H, van den Born M, Korving J, de Sauvage F, et al. Replacement of Lost Lgr5-Positive Stem Cells through Plasticity of Their Enterocyte-Lineage Daughters. Cell Stem Cell. 2016 Feb 4;18:203-13.

59. Tetteh PW, Farin HF, Clevers H. Plasticity within stem cell hierarchies in mammalian epithelia. Trends Cell Biol. 2015 Feb;25:100-8.

60. Beyaz S, Mana MD, Roper J, Kedrin D, Saadatpour A, Hong SJ, Bauer-Rowe KE, Xifaras ME, Akkad A, et al. High-fat diet enhances stemness and tumorigenicity of intestinal progenitors. Nature. 2016 Mar 3;531:53-8.

61. DeClercq V, McMurray DN, Chapkin RS. Obesity promotes colonic stem cell expansion during cancer initiation. Cancer Lett. 2015 Dec 28;369:336-43.

62. Igarashi M, Guarente L. mTORC1 and SIRT1 Cooperate to Foster Expansion of Gut Adult Stem Cells during Calorie Restriction. Cell. 2016 Jul 14;166:436-50.

63. Kim E, Davidson LA, Zoh RS, Hensel ME, Salinas ML, Patil BS, Jayaprakasha GK, Callaway ES, Allred CD, et al. Rapidly cycling Lgr5(+) stem cells are exquisitely sensitive to extrinsic dietary factors that modulate colon cancer risk. Cell Death Dis. 2016 Nov 10;7:e2460.

64. Chang WCL, Chapkin RS, Lupton JR. Predictive value of proliferation, differentiation and apoptosis as intermediate markers for colon tumorigenesis. Carcinogenesis. 1997 Apr;18:721-30.

65. Ward JM, Uno H, Kurata Y, Weghorst CM, Jang JJ. Cell proliferation not associated with carcinogenesis in rodents and humans. Environ Health Perspect. 1993;101:125-35.

66. Reed JC. Mechanisms of apoptosis. Am J Pathol. 2000 Nov;157:1415-30.

67. Fulda S, Debatin KM. Extrinsic versus intrinsic apoptosis pathways in anticancer chemotherapy. Oncogene. 2006 Aug 7;25:4798-811.

68. Saelens X, Festjens N, Vande Walle L, van Gurp M, van Loo G, Vandenabeele P. Toxic proteins released from mitochondria in cell death. Oncogene. 2004 Apr 12;23:2861-74.

69. Ichim G, Tait SW. A fate worse than death: apoptosis as an oncogenic process. Nature Reviews Cancer. 2016;16:539.

70. Green DR, Kroemer G. The pathophysiology of mitochondrial cell death. Science. 2004 Jul 30;305:626-9. 
71. Ng Y, Barhoumi R, Tjalkens RB, Fan YY, Kolar S, Wang N, Lupton JR, Chapkin RS. The role of docosahexaenoic acid in mediating mitochondrial membrane lipid oxidation and apoptosis in colonocytes. Carcinogenesis. 2005 Nov;26:1914-21.

72. Rodrigues NR, Rowan A, Smith ME, Kerr IB, Bodmer WF, Gannon JV, Lane DP. p53 mutations in colorectal cancer. Proc Natl Acad Sci U S A. 1990 Oct;87:7555-9.

73. Cho Y, Turner ND, Davidson LA, Chapkin RS, Carroll RJ, Lupton JR. A chemoprotective fish oil/pectin diet enhances apoptosis via Bcl-2 promoter methylation in rat azoxymethane-induced carcinomas. Exp Biol Med (Maywood). 2012 Dec;237:1387-93.

74. Hong MY, Chapkin RS, Davidson LA, Turner ND, Morris JS, Carroll RJ, Lupton JR. Fish oil enhances targeted apoptosis during colon tumor initiation in part by downregulating Bcl2. Nutr Cancer. 2003;46:44-51.

75. Wright SC, Zhong J, Larrick JW. Inhibition of Apoptosis as a Mechanism of Tumor Promotion. FASEB J. 1994 Jun;8:654-60.

76. Herman JG, Umar A, Polyak K, Graff JR, Ahuja N, Issa JP, Markowitz S, Willson JK, Hamilton SR, et al. Incidence and functional consequences of hMLH1 promoter hypermethylation in colorectal carcinoma. Proc Natl Acad Sci U S A. 1998 Jun 9;95:6870-5.

77. Cho Y, Turner ND, Davidson LA, Chapkin RS, Carroll RJ, Lupton JR. Colon cancer cell apoptosis is induced by combined exposure to the $n-3$ fatty acid docosahexaenoic acid and butyrate through promoter methylation. Exp Biol Med (Maywood). 2014 Mar;239:302-10.

78. Glebov OK, Rodriguez LM, Nakahara K, Jenkins J, Cliatt J, Humbyrd CJ, DeNobile J, Soballe P, Simon R, et al. Distinguishing right from left colon by the pattern of gene expression. Cancer Epidemiol Biomarkers Prev. 2003 Aug;12:755-62.

79. Barnicle A, Seoighe C, Golden A, Greally JM, Egan LJ. Differential DNA methylation patterns of homeobox genes in proximal and distal colon epithelial cells. Physiol Genomics. 2016 Apr;48:257-73.

80. Minoo P, Zlobec I, Peterson M, Terracciano L, Lugli A. Characterization of rectal, proximal and distal colon cancers based on clinicopathological, molecular and protein profiles. Int J Oncol. 2010 Sep;37:707-18.

81. Yamauchi M, Morikawa T, Kuchiba A, Imamura Y, Qian ZR, Nishihara R, Liao X, Waldron L, Hoshida Y, et al. Assessment of colorectal cancer molecular features along bowel subsites challenges the conception of distinct dichotomy of proximal versus distal colorectum. Gut. 2012 Jun;61:847-54.

82. Watanabe T, Kobunai T, Toda E, Yamamoto Y, Kanazawa T, Kazama Y, Tanaka J, Tanaka T, Konishi T, et al. Distal colorectal cancers with microsatellite instability (MSI) display distinct gene expression profiles that are different from proximal MSI cancers. Cancer Res. 2006 Oct 15;66:9804-8. 
83. Hong MY, Chang WCL, Chapkin RS, Lupton JR. Relationship among colonocyte proliferation, differentiation, and apoptosis as a function of diet and carcinogen. Nutrition and Cancer-an International Journal. 1997;28:20-9.

84. Donaldson GP, Lee SM, Mazmanian SK. Gut biogeography of the bacterial microbiota. Nat Rev Microbiol. 2016 Jan;14:20-32.

85. Wiseman H, Halliwell B. Damage to DNA by reactive oxygen and nitrogen species: role in inflammatory disease and progression to cancer. Biochem J. 1996 Jan 1;313 ( Pt 1):17-29.

86. Meira LB, Bugni JM, Green SL, Lee CW, Pang B, Borenshtein D, Rickman BH, Rogers $\mathrm{AB}$, Moroski-Erkul CA, et al. DNA damage induced by chronic inflammation contributes to colon carcinogenesis in mice. J Clin Invest. 2008 Jul;118:2516-25.

87. Hahn MA, Hahn T, Lee DH, Esworthy RS, Kim BW, Riggs AD, Chu FF, Pfeifer GP. Methylation of polycomb target genes in intestinal cancer is mediated by inflammation. Cancer Res. 2008 Dec 15;68:10280-9.

88. Iliopoulos D, Jaeger SA, Hirsch HA, Bulyk ML, Struhl K. STAT3 activation of miR-21 and miR-181b-1 via PTEN and CYLD are part of the epigenetic switch linking inflammation to cancer. Mol Cell. 2010 Aug 27;39:493-506.

89. Colotta F, Allavena P, Sica A, Garlanda C, Mantovani A. Cancer-related inflammation, the seventh hallmark of cancer: links to genetic instability. Carcinogenesis. 2009 Jul;30:1073-81.

90. Edwards RA, Witherspoon M, Wang K, Afrasiabi K, Pham T, Birnbaumer L, Lipkin SM. Epigenetic repression of DNA mismatch repair by inflammation and hypoxia in inflammatory bowel disease-associated colorectal cancer. Cancer Res. 2009 Aug 15;69:6423-9.

91. Vannucci L, Stepankova R, Kozakova H, Fiserova A, Rossmann P, TlaskalovaHogenova $\mathrm{H}$. Colorectal carcinogenesis in germ-free and conventionally reared rats: different intestinal environments affect the systemic immunity. Int J Oncol. 2008 Mar;32:609-17.

92. Sacksteder MR. Occurrence of spontaneous tumors in the germfree F344 rat. J Natl Cancer Inst. 1976 Dec;57:1371-3.

93. Reddy BS, Weisburger JH, Narisawa T, Wynder EL. Colon carcinogenesis in germ-free rats with 1,2-dimethylhydrazine and N-methyl-n'-nitro-N-nitrosoguanidine. Cancer Res. 1974 Sep;34:2368-72.

94. Reddy BS, Narisawa T, Wright P, Vukusich D, Weisburger JH, Wynder EL. Colon carcinogenesis with azoxymethane and dimethylhydrazine in germ-free rats. Cancer Res. 1975 Feb;35:287-90.

95. Uronis JM, Muhlbauer M, Herfarth HH, Rubinas TC, Jones GS, Jobin C. Modulation of the intestinal microbiota alters colitis-associated colorectal cancer susceptibility. PLoS One. 2009 Jun 24;4:e6026. 
96. Grivennikov SI, Wang K, Mucida D, Stewart CA, Schnabl B, Jauch D, Taniguchi K, Yu $\mathrm{GY}$, Osterreicher $\mathrm{CH}$, et al. Adenoma-linked barrier defects and microbial products drive IL23/IL-17-mediated tumour growth. Nature. 2012 Nov 8;491:254-8.

97. Klimesova K, Kverka M, Zakostelska Z, Hudcovic T, Hrncir T, Stepankova R, Rossmann P, Ridl J, Kostovcik M, et al. Altered gut microbiota promotes colitis-associated cancer in IL-1 receptor-associated kinase M-deficient mice. Inflamm Bowel Dis. 2013 May;19:1266-77.

98. Garaude J, Kent A, van Rooijen N, Blander JM. Simultaneous targeting of toll- and nodlike receptors induces effective tumor-specific immune responses. Sci Transl Med. 2012 Feb $8 ; 4: 120$ ra16.

99. Pradere JP, Dapito DH, Schwabe RF. The Yin and Yang of Toll-like receptors in cancer. Oncogene. 2014 Jul 3;33:3485-95.

100. Ott SJ, Musfeldt M, Wenderoth DF, Hampe J, Brant O, Folsch UR, Timmis KN, Schreiber S. Reduction in diversity of the colonic mucosa associated bacterial microflora in patients with active inflammatory bowel disease. Gut. 2004 May;53:685-93.

101. Manichanh C, Rigottier-Gois L, Bonnaud E, Gloux K, Pelletier E, Frangeul L, Nalin R, Jarrin C, Chardon P, et al. Reduced diversity of faecal microbiota in Crohn's disease revealed by a metagenomic approach. Gut. 2006 Feb;55:205-11.

102. Sepehri S, Kotlowski R, Bernstein CN, Krause DO. Microbial diversity of inflamed and noninflamed gut biopsy tissues in inflammatory bowel disease. Inflamm Bowel Dis. 2007 Jun;13:675-83.

103. Ritchie LE, Sturino JM, Carroll RJ, Rooney LW, Azcarate-Peril MA, Turner ND. Polyphenol-rich sorghum brans alter colon microbiota and impact species diversity and species richness after multiple bouts of dextran sodium sulfate-induced colitis. FEMS Microbiol Ecol. 2015 Mar;91:fiv008.

104. Ritchie LE, Taddeo SS, Weeks BR, Lima F, Bloomfield SA, Azcarate-Peril MA, Zwart SR, Smith SM, Turner ND. Space Environmental Factor Impacts upon Murine Colon Microbiota and Mucosal Homeostasis. PLoS One. 2015 Jun 17;10:e0125792.

105. Wang T, Cai G, Qiu Y, Fei N, Zhang M, Pang X, Jia W, Cai S, Zhao L. Structural segregation of gut microbiota between colorectal cancer patients and healthy volunteers. The ISME journal. 2012;6:320.

106. Wu N, Yang X, Zhang R, Li J, Xiao X, Hu Y, Chen Y, Yang F, Lu N, et al. Dysbiosis signature of fecal microbiota in colorectal cancer patients. Microb Ecol. 2013 Aug;66:462-70.

107. Mira-Pascual L, Cabrera-Rubio R, Ocon S, Costales P, Parra A, Suarez A, Moris F, Rodrigo L, Mira A, Collado MC. Microbial mucosal colonic shifts associated with the development of colorectal cancer reveal the presence of different bacterial and archaeal biomarkers. J Gastroenterol. 2015 Feb;50:167-79. 
108. Chen W, Liu F, Ling Z, Tong X, Xiang C. Human intestinal lumen and mucosaassociated microbiota in patients with colorectal cancer. PLoS One. 2012;7:e39743.

109. Weir TL, Manter DK, Sheflin AM, Barnett BA, Heuberger AL, Ryan EP. Stool microbiome and metabolome differences between colorectal cancer patients and healthy adults. PLoS One. 2013;8:e70803.

110. Sanchez-Espiridion B, White L, Mishra L, Raju GS, Kopetz S, Gu J, Ye Y, Wu X, Liang D. Abstract 8: Global and targeted metabolomic profiling of colorectal cancer progression. Cancer Res. 2016;76:8-.

111. Brown DG, Rao S, Weir TL, O'Malia J, Bazan M, Brown RJ, Ryan EP. Metabolomics and metabolic pathway networks from human colorectal cancers, adjacent mucosa, and stool. Cancer Metab. 2016;4:11.

112. Gagnaire A, Nadel B, Raoult D, Neefjes J, Gorvel JP. Collateral damage: insights into bacterial mechanisms that predispose host cells to cancer. Nat Rev Microbiol. 2017 Feb;15:10928.

113. Vizcaino MI, Crawford JM. The colibactin warhead crosslinks DNA. Nat Chem. 2015 May;7:411-7.

114. Wu S, Powell J, Mathioudakis N, Kane S, Fernandez E, Sears CL. Bacteroides fragilis enterotoxin induces intestinal epithelial cell secretion of interleukin- 8 through mitogen-activated protein kinases and a tyrosine kinase-regulated nuclear factor- $\kappa \mathrm{B}$ pathway. Infect Immun. 2004;72:5832-9.

115. Wu S, Shin J, Zhang G, Cohen M, Franco A, Sears CL. The Bacteroides fragilis toxin binds to a specific intestinal epithelial cell receptor. Infect Immun. 2006 Sep;74:5382-90.

116. Goodwin AC, Destefano Shields CE, Wu S, Huso DL, Wu X, Murray-Stewart TR, Hacker-Prietz A, Rabizadeh S, Woster PM, et al. Polyamine catabolism contributes to enterotoxigenic Bacteroides fragilis-induced colon tumorigenesis. Proc Natl Acad Sci U S A. 2011 Sep 13;108:15354-9.

117. Boleij A, Hechenbleikner EM, Goodwin AC, Badani R, Stein EM, Lazarev MG, Ellis B, Carroll KC, Albesiano E, et al. The Bacteroides fragilis toxin gene is prevalent in the colon mucosa of colorectal cancer patients. Clin Infect Dis. 2015 Jan 15;60:208-15.

118. Lu R, Wu S, Zhang YG, Xia Y, Zhou Z, Kato I, Dong H, Bissonnette M, Sun J. Salmonella Protein AvrA Activates the STAT3 Signaling Pathway in Colon Cancer. Neoplasia. 2016 May;18:307-16.

119. Corvinus FM, Orth C, Moriggl R, Tsareva SA, Wagner S, Pfitzner EB, Baus D, Kaufman R, Huber LA, et al. Persistent STAT3 Activation in Colon Cancer Is Associated with Enhanced Cell Proliferation and Tumor Growth. Neoplasia. 2005 Jun;7:545-55. 
120. Lin L, Liu A, Peng Z, Lin HJ, Li PK, Li C, Lin J. STAT3 is necessary for proliferation and survival in colon cancer-initiating cells. Cancer Res. 2011 Dec 1;71:7226-37.

121. Grivennikov S, Karin E, Terzic J, Mucida D, Yu GY, Vallabhapurapu S, Scheller J, Rose-John S, Cheroutre H, et al. IL-6 and Stat3 are required for survival of intestinal epithelial cells and development of colitis-associated cancer. Cancer Cell. 2009 Feb 3;15:103-13.

122. Bromberg J, Wang TC. Inflammation and cancer: IL-6 and STAT3 complete the link. Cancer Cell. 2009 Feb 3;15:79-80.

123. Raisch J, Rolhion N, Dubois A, Darfeuille-Michaud A, Bringer MA. Intracellular colon cancer-associated Escherichia coli promote protumoral activities of human macrophages by inducing sustained COX-2 expression. Lab Invest. 2015 Mar;95:296-307.

124. Wang X, Huycke MM. Colorectal cancer: role of commensal bacteria and bystander effects. Gut Microbes. 2015 Nov 2;6:370-6.

125. Hall EJ, Giaccia AJ. Radiobiology for the Radiologist: Lippincott Williams \& Wilkins Philadelphia; 2006.

126. Task Group on Radiation Protection in Space IC, Dietze G, Bartlett DT, Cool DA, Cucinotta FA, Jia X, McAulay IR, Pelliccioni M, Petrov V, et al. ICRP, 123. Assessment of radiation exposure of astronauts in space. ICRP Publication 123. Ann ICRP. 2013 Aug;42:1-339.

127. Riley P. Free radicals in biology: oxidative stress and the effects of ionizing radiation. International journal of radiation biology. 1994;65:27-33.

128. Pouget J-P, Mather SJ. General aspects of the cellular response to low- and high-LET radiation. European Journal of Nuclear Medicine. 2001;28:541-61.

129. Zeitlin C, Hassler DM, Cucinotta FA, Ehresmann B, Wimmer-Schweingruber RF, Brinza DE, Kang S, Weigle G, Bottcher S, et al. Measurements of energetic particle radiation in transit to Mars on the Mars Science Laboratory. Science. 2013 May 31;340:1080-4.

130. Cucinotta FA, Kim M-HY, Chappell LJ. Probability of causation for space radiation carcinogenesis following international space station, near Earth asteroid, and Mars missions. 2012 .

131. Anson NM, Selinheimo E, Havenaar R, Aura AM, Mattila I, Lehtinen P, Bast A, Poutanen K, Haenen GR. Bioprocessing of wheat bran improves in vitro bioaccessibility and colonic metabolism of phenolic compounds. J Agric Food Chem. 2009 Jul 22;57:6148-55.

132. Hemery YM, Anson NM, Havenaar R, Haenen GR, Noort MW, Rouau X. Dryfractionation of wheat bran increases the bioaccessibility of phenolic acids in breads made from processed bran fractions. Food Research International. 2010;43:1429-38. 
133. Maciorowski KG, Turner ND, Lupton JR, Chapkin RS, Shermer CL, Ha SD, Ricke SC. Diet and carcinogen alter the fecal microbial populations of rats. J Nutr. 1997 Mar;127:449-57.

134. Taguri T, Tanaka T, Kouno I. Antimicrobial activity of 10 different plant polyphenols against bacteria causing food-borne disease. Biol Pharm Bull. 2004 Dec;27:1965-9.

135. Sójka M, Kołodziejczyk K, Milala J, Abadias M, Viñas I, Guyot S, Baron A.

Composition and properties of the polyphenolic extracts obtained from industrial plum pomaces. J Funct Foods. 2015 Jan;12:168-78.

136. O'Keefe SJ, Li JV, Lahti L, Ou J, Carbonero F, Mohammed K, Posma JM, Kinross J, Wahl E, Ruder E. Fat, fibre and cancer risk in African Americans and rural Africans. Nature communications. 2015;6.

137. Zhang Z, Geng J, Tang X, Fan H, Xu J, Wen X, Ma ZS, Shi P. Spatial heterogeneity and co-occurrence patterns of human mucosal-associated intestinal microbiota. ISME J. 2014 Apr;8:881-93.

138. Consortium HMP. Structure, function and diversity of the healthy human microbiome. Nature. 2012;486:207-14.

139. Clifford M. Diet-derived phenols in plasma and tissues and their implications for health. Planta Med. 2004;70:1103-14.

140. Williamson G, Clifford MN. Colonic metabolites of berry polyphenols: the missing link to biological activity? Br J Nutr. 2010 Oct;104 Suppl 3:S48-66.

141. Zhang LS, Davies SS. Microbial metabolism of dietary components to bioactive metabolites: opportunities for new therapeutic interventions. Genome Med. 2016 Apr 21;8:46.

142. Cheng Y, Jin UH, Allred CD, Jayaraman A, Chapkin RS, Safe S. Aryl Hydrocarbon Receptor Activity of Tryptophan Metabolites in Young Adult Mouse Colonocytes. Drug Metab Dispos. 2015 Oct;43:1536-43.

143. Louis P, Hold GL, Flint HJ. The gut microbiota, bacterial metabolites and colorectal cancer. Nat Rev Microbiol. 2014 Oct;12:661-72.

144. Turner ND, Lupton JR. Dietary fiber. Adv Nutr. 2011 Mar;2:151-2.

145. Macfarlane GT, Macfarlane S. Human colonic microbiota: ecology, physiology and metabolic potential of intestinal bacteria. Scand J Gastroenterol Suppl. 1997;222:3-9.

146. Lupton JR, Kurtz PP. Relationship of colonic luminal short-chain fatty acids and $\mathrm{pH}$ to in vivo cell proliferation in rats. J Nutr. 1993 Sep;123:1522-30.

147. Cummings JH, Pomare EW, Branch WJ, Naylor CP, Macfarlane GT. Short chain fatty acids in human large intestine, portal, hepatic and venous blood. Gut. 1987 Oct;28:1221-7. 
148. Hamer HM, Jonkers D, Venema K, Vanhoutvin S, Troost FJ, Brummer RJ. Review article: the role of butyrate on colonic function. Aliment Pharmacol Ther. 2008 Jan 15;27:10419.

149. Lupton JR. Microbial degradation products influence colon cancer risk: the butyrate controversy. J Nutr. 2004 Feb;134:479-82.

150. Lupton JR, Turner ND. Potential protective mechanisms of wheat bran fiber. Am J Med. 1999 Jan 25;106:24S-7S.

151. Gazzaniga JM, Lupton JR. Dilution effect of dietary fiber sources: an in vivo study in the rat. Nutr Res. 1987;7:1261-8.

152. Lewis SJ, Heaton KW. Increasing butyrate concentration in the distal colon by accelerating intestinal transit. Gut. 1997 Aug;41:245-51.

153. Zoran DL, Turner ND, Taddeo SS, Chapkin RS, Lupton JR. Wheat bran diet reduces tumor incidence in a rat model of colon cancer independent of effects on distal luminal butyrate concentrations. J Nutr. 1997 Nov;127:2217-25.

154. Tudela CV, Boudry C, Stumpff F, Aschenbach JR, Vahjen W, Zentek J, Pieper R. Downregulation of monocarboxylate transporter 1 (MCT1) gene expression in the colon of piglets is linked to bacterial protein fermentation and pro-inflammatory cytokine-mediated signalling. $\mathrm{Br} \mathbf{J}$ Nutr. 2015;113:610-7.

155. Liu X, Blouin J-M, Santacruz A, Lan A, Andriamihaja M, Wilkanowicz S, Benetti P-H, Tomé D, Sanz Y, Blachier F. High-protein diet modifies colonic microbiota and luminal environment but not colonocyte metabolism in the rat model: the increased luminal bulk connection. American Journal of Physiology-Gastrointestinal and Liver Physiology. 2014;307:G459-G70.

156. Donohoe DR, Curry KP, Bultman SJ. Microbial oncotarget: bacterial-produced butyrate, chemoprevention and Warburg effect. Oncotarget. 2013 Feb;4:182-3.

157. Vital M, Howe AC, Tiedje JM. Revealing the bacterial butyrate synthesis pathways by analyzing (meta)genomic data. MBio. 2014 Apr 22;5:e00889.

158. Donohoe DR, Collins LB, Wali A, Bigler R, Sun W, Bultman SJ. The Warburg effect dictates the mechanism of butyrate-mediated histone acetylation and cell proliferation. Mol Cell. 2012 Nov 30;48:612-26.

159. Zimmerman MA, Singh N, Martin PM, Thangaraju M, Ganapathy V, Waller JL, Shi H, Robertson KD, Munn DH, Liu K. Butyrate suppresses colonic inflammation through HDAC1dependent Fas upregulation and Fas-mediated apoptosis of T cells. Am J Physiol Gastrointest Liver Physiol. 2012 Jun 15;302:G1405-15. 
160. Ganapathy V, Gurav A, Singh N. The butyrate transporter SLC5A8 is a tumor suppressor in colon linked to dietary fiber content. AACR; 2015.

161. Gurav A, Sivaprakasam S, Bhutia YD, Boettger T, Singh N, Ganapathy V. Slc5a8, a $\mathrm{Na}$-coupled high-affinity transporter for short-chain fatty acids, is a conditional tumour suppressor in colon that protects against colitis and colon cancer under low-fibre dietary conditions. Biochem J. 2015;469:267-78.

162. Gurav A, Singh N, Ganapathy V. A critical role for Slc5a8 in the suppression of colonic inflammation by commensal bacteria-derived metabolites (MUC9P. 821). The Journal of Immunology. 2014;192:199.8.

163. Fan YY, Zhang J, Barhoumi R, Burghardt RC, Turner ND, Lupton JR, Chapkin RS. Antagonism of CD95 signaling blocks butyrate induction of apoptosis in young adult mouse colonic cells. Am J Physiol. 1999 Aug;277:C310-9.

164. Chapkin RS, Fan Y-Y, Lupton JR. Effect of diet on colonic-programmed cell death: molecular mechanism of action. Toxicol Lett. 2000;112:411-4.

165. Kim M, Qie Y, Park J, Kim CH. Gut Microbial Metabolites Fuel Host Antibody Responses. Cell Host Microbe. 2016 Aug 10;20:202-14.

166. Furusawa Y, Obata Y, Hase K. Commensal microbiota regulates T cell fate decision in the gut. Semin Immunopathol; 2015: Springer; 2015. p. 17-25.

167. Crim KC, Sanders LM, Hong MY, Taddeo SS, Turner ND, Chapkin RS, Lupton JR. Upregulation of $\mathrm{p} 21 \mathrm{Waf} 1 / \mathrm{Cip} 1$ expression in vivo by butyrate administration can be chemoprotective or chemopromotive depending on the lipid component of the diet. Carcinogenesis. 2008 Jul;29:1415-20.

168. Belcheva A, Irrazabal T, Martin A. Gut microbial metabolism and colon cancer: can manipulations of the microbiota be useful in the management of gastrointestinal health? Bioessays. 2015 Apr;37:403-12.

169. Belcheva A, Irrazabal T, Robertson SJ, Streutker C, Maughan H, Rubino S, Moriyama EH, Copeland JK, Surendra A, et al. Gut microbial metabolism drives transformation of MSH2deficient colon epithelial cells. Cell. 2014 Jul 17;158:288-99.

170. Liu RH. Health benefits of fruit and vegetables are from additive and synergistic combinations of phytochemicals. Am J Clin Nutr. 2003 Sep;78:517S-20S.

171. Tsao R, Yang R, Young JC, Zhu H. Polyphenolic profiles in eight apple cultivars using high-performance liquid chromatography (HPLC). J Agric Food Chem. 2003;51:6347-53.

172. Vanamala J, Cobb G, Turner ND, Lupton JR, Yoo KS, Pike LM, Patil BS. Bioactive compounds of grapefruit (Citrus paradisi $\mathrm{Cv}$. Rio Red) respond differently to postharvest irradiation, storage, and freeze drying. J Agric Food Chem. 2005 May 18;53:3980-5. 
173. Dinkova-Kostova AT. Phytochemicals as protectors against ultraviolet radiation: versatility of effects and mechanisms. Planta Med. 2008;74:1548-59.

174. Alothman M, Bhat R, Karim A. Effects of radiation processing on phytochemicals and antioxidants in plant produce. Trends in Food Science \& Technology. 2009;20:201-12.

175. Holt EM, Steffen LM, Moran A, Basu S, Steinberger J, Ross JA, Hong CP, Sinaiko AR. Fruit and vegetable consumption and its relation to markers of inflammation and oxidative stress in adolescents. J Am Diet Assoc. 2009 Mar;109:414-21.

176. Siriwardhana N, Kalupahana NS, Cekanova M, LeMieux M, Greer B, Moustaid-Moussa N. Modulation of adipose tissue inflammation by bioactive food compounds. J Nutr Biochem. 2013 Apr;24:613-23.

177. Cowan MM. Plant products as antimicrobial agents. Clin Microbiol Rev. 1999;12:56482.

178. Scalbert A, Williamson G. Dietary intake and bioavailability of polyphenols. J Nutr. 2000 Aug;130:2073S-85S.

179. Dykes. Phenolic Compounds in Cereal Grains and Their Health Benefits. Cereal Foods World. 2007 May-Jun;52:105-11.

180. Vanamala J, Leonardi T, Patil BS, Taddeo SS, Murphy ME, Pike LM, Chapkin RS, Lupton JR, Turner ND. Suppression of colon carcinogenesis by bioactive compounds in grapefruit. Carcinogenesis. 2006 Jun;27:1257-65.

181. Leonardi T, Vanamala J, Taddeo SS, Davidson LA, Murphy ME, Patil BS, Wang N, Carroll RJ, Chapkin RS, et al. Apigenin and naringenin suppress colon carcinogenesis through the aberrant crypt stage in azoxymethane-treated rats. Exp Biol Med (Maywood). 2010 Jun;235:710-7.

182. Yang CS, Landau JM, Huang MT, Newmark HL. Inhibition of carcinogenesis by dietary polyphenolic compounds. Annu Rev Nutr. 2001;21:381-406.

183. Lea MA, Ibeh C, desBordes C, Vizzotto M, Cisneros-Zevallos L, Byrne DH, Okie WR, Moyer MP. Inhibition of growth and induction of differentiation of colon cancer cells by peach and plum phenolic compounds. Anticancer Res. 2008 Jul-Aug;28:2067-76.

184. Lea MA, Ibeh C, Han L, Desbordes C. Inhibition of growth and induction of differentiation markers by polyphenolic molecules and histone deacetylase inhibitors in colon cancer cells. Anticancer Res. 2010 Feb;30:311-8.

185. Miene C, Weise A, Glei M. Impact of polyphenol metabolites produced by colonic microbiota on expression of COX-2 and GSTT2 in human colon cells (LT97). Nutr Cancer. 2011;63:653-62. 
186. Link A, Balaguer F, Goel A. Cancer chemoprevention by dietary polyphenols: promising role for epigenetics. Biochem Pharmacol. 2010 Dec 15;80:1771-92.

187. Banerjee N, Kim H, Talcott ST, Turner ND, Byrne DH, Mertens-Talcott SU. Plum polyphenols inhibit colorectal aberrant crypt foci formation in rats: potential role of the miR143/protein kinase B/mammalian target of rapamycin axis. Nutr Res. 2016 Oct;36:1105-13.

188. Shankar E, Kanwal R, Candamo M, Gupta S. Dietary phytochemicals as epigenetic modifiers in cancer: promise and challenges. Semin Cancer Biol. 2016;40:82-99.

189. Rechner AR, Smith MA, Kuhnle G, Gibson GR, Debnam ES, Srai SK, Moore KP, RiceEvans CA. Colonic metabolism of dietary polyphenols: influence of structure on microbial fermentation products. Free Radic Biol Med. 2004 Jan 15;36:212-25.

190. Déprez S, Brezillon C, Rabot S, Philippe C, Mila I, Lapierre C, Scalbert A. Polymeric Proanthocyanidins Are Catabolized by Human Colonic Microflora into Low-Molecular-Weight Phenolic Acids. The Journal of Nutrition. 2000 Nov 1;130:2733-8.

191. Cardona F, Andres-Lacueva C, Tulipani S, Tinahones FJ, Queipo-Ortuno MI. Benefits of polyphenols on gut microbiota and implications in human health. J Nutr Biochem. 2013 Aug;24:1415-22.

192. Duenas M, Munoz-Gonzalez I, Cueva C, Jimenez-Giron A, Sanchez-Patan F, SantosBuelga C, Moreno-Arribas MV, Bartolome B. A survey of modulation of gut microbiota by dietary polyphenols. Biomed Res Int. 2015;850902.

193. Etxeberria U, Fernandez-Quintela A, Milagro FI, Aguirre L, Martinez JA, Portillo MP. Impact of polyphenols and polyphenol-rich dietary sources on gut microbiota composition. J Agric Food Chem. 2013 Oct 9;61:9517-33.

194. Stacewicz-Sapuntzakis M, Bowen PE, Hussain EA, Damayanti-Wood BI, Farnsworth NR. Chemical composition and potential health effects of prunes: a functional food? Crit Rev Food Sci Nutr. 2001 May;41:251-86.

195. Fang N, Yu S, Prior RL. LC/MS/MS characterization of phenolic constituents in dried plums. J Agric Food Chem. 2002 Jun 5;50:3579-85.

196. Piefer LA, Weeks B, Carroll R, Byrne D, Ambrus A, Turner N. Quercetin and chlorogenic acid affect butyrate excretion, NF- $\kappa \mathrm{B}$ activity, and cell proliferation in DSS treated rats. The FASEB Journal. 2012;26:263.6-.6.

197. Turner N, Paulhill K, Warren C, Davidson L, Chapkin R, Lupton J, Carroll R, Wang N. Quercetin suppresses early colon carcinogenesis partly through inhibition of inflammatory mediators. II International Symposium on Human Health Effects of Fruits and Vegetables: FAVHEALTH. 2007;841;237-42. 
198. Warren CA, Paulhill KJ, Davidson LA, Lupton JR, Taddeo SS, Hong MY, Carroll RJ, Chapkin RS, Turner ND. Quercetin may suppress rat aberrant crypt foci formation by suppressing inflammatory mediators that influence proliferation and apoptosis. The Journal of nutrition. 2009; 139:101-5.

199. Zhang XA, Zhang S, Yin Q, Zhang J. Quercetin induces human colon cancer cells apoptosis by inhibiting the nuclear factor-kappa B Pathway. Pharmacogn Mag. 2015 Apr;11:404-9.

200. Refolo MG, D'Alessandro R, Malerba N, Laezza C, Bifulco M, Messa C, Caruso MG, Notarnicola M, Tutino V. Anti Proliferative and Pro Apoptotic Effects of Flavonoid Quercetin Are Mediated by CB1 Receptor in Human Colon Cancer Cell Lines. J Cell Physiol. 2015 Dec;230:2973-80.

201. Han M, Song Y, Zhang X. Quercetin Suppresses the Migration and Invasion in Human Colon Cancer Caco-2 Cells Through Regulating Toll-like Receptor 4/Nuclear Factor-kappa B Pathway. Pharmacogn Mag. 2016 May;12:S237-44.

202. Dihal AA, de Boer VC, van der Woude H, Tilburgs C, Bruijntjes JP, Alink GM, Rietjens IM, Woutersen RA, Stierum RH. Quercetin, but not its glycosidated conjugate rutin, inhibits azoxymethane-induced colorectal carcinogenesis in F344 rats. The Journal of nutrition. 2006;136:2862-7.

203. Peters R, Lock RH. Laxative effect of sorbitol. Br Med J. 1958 Sep 13;2:677-8.

204. Lu X, Li C, Wang YK, Jiang K, Gai XD. Sorbitol induces apoptosis of human colorectal cancer cells via p38 MAPK signal transduction. Oncol Lett. 2014 Jun;7:1992-6.

205. Yang Y, Gallaher DD. Effect of dried plums on colon cancer risk factors in rats. Nutr Cancer. 2005 2005/09/01;53:117-25.

206. Glinghammar B, Inoue H, Rafter JJ. Deoxycholic acid causes DNA damage in colonic cells with subsequent induction of caspases, COX-2 promoter activity and the transcription factors NF-kB and AP-1. Carcinogenesis. 2002 May;23:839-45.

207. Baijal PK, Clow EP, Fitzpatrick DW, Bird RP. Tumor-enhancing effects of cholic acid are exerted on the early stages of colon carcinogenesis via induction of aberrant crypt foci with an enhanced growth phenotype. Can J Physiol Pharmacol. 1998 Dec;76:1095-102.

208. Liu RH. Whole grain phytochemicals and health. Journal of Cereal Science. 2007 Nov;46:207-19.

209. Dykes L, Rooney LW. Sorghum and millet phenols and antioxidants. Journal of Cereal Science. 2006 Nov;44:236-51.

210. Awika JM, McDonough CM, Rooney LW. Decorticating sorghum to concentrate healthy phytochemicals. J Agric Food Chem. 2005 Aug 10;53:6230-4. 
211. Ritchie L, Taddeo S, Weeks B, Carroll R, Dykes L, Rooney L, Turner N. Impact of Novel Sorghum Bran Diets on DSS-Induced Colitis. Nutrients. 2017;9:330.

212. Ritchie LE, Sturino JM, Azcarate-Peril MA, Turner ND. Novel sorghum brans containing bioactive compounds alter colon microbiota in response to a DSS-induced chronic inflammatory state. FASEB J. 2013 Apr;27:247.2.

213. Huttenhower C, Gevers D, Knight R, Abubucker S, Badger JH, Chinwalla AT, Creasy HH, Earl AM, FitzGerald MG, Fulton RS. Structure, function and diversity of the healthy human microbiome. Nature. 2012;486:207.

214. Turnbaugh PJ, Hamady M, Yatsunenko T, Cantarel BL, Duncan A, Ley RE, Sogin ML, Jones WJ, Roe BA, et al. A core gut microbiome in obese and lean twins. Nature. 2009 Jan 22;457:480-4.

215. Xu Z, Malmer D, Langille MG, Way SF, Knight R. Which is more important for classifying microbial communities: who's there or what they can do? ISME J. 2014 Dec;8:23579 .

216. Integrative HMPRNC. The Integrative Human Microbiome Project: dynamic analysis of microbiome-host omics profiles during periods of human health and disease. Cell Host Microbe. 2014 Sep 10;16:276-89.

217. O'toole PW, Flemer B. Studying the Microbiome:“Omics” Made Accessible. Semin Liver Dis; 2016 Sep;36:306-11.

218. Weinstock GM. Genomic approaches to studying the human microbiota. Nature. 2012 Sep 13;489:250-6.

219. Kuczynski J, Stombaugh J, Walters WA, González A, Caporaso JG, Knight R. Using QIIME to Analyze 16S rRNA Gene Sequences from Microbial Communities. Curr Protoc Microbiol. 2012 Nov;27:1E-5.

220. Langille MG, Zaneveld J, Caporaso JG, McDonald D, Knights D, Reyes JA, Clemente JC, Burkepile DE, Vega Thurber RL, et al. Predictive functional profiling of microbial communities using 16S rRNA marker gene sequences. Nat Biotechnol. 2013 Sep;31:814-21.

221. Kanehisa M, Goto S. KEGG: kyoto encyclopedia of genes and genomes. Nucleic Acids Res. 2000 Jan 1;28:27-30.

222. Ashburner M, Ball CA, Blake JA, Botstein D, Butler H, Cherry JM, Davis AP, Dolinski $\mathrm{K}$, Dwight SS, et al. Gene ontology: tool for the unification of biology. The Gene Ontology Consortium. Nat Genet. 2000 May;25:25-9.

223. Siegel R, Desantis C, Jemal A. Colorectal cancer statistics, 2014. CA Cancer J Clin. 2014 Mar;64:104-17. 
224. Boursi B, Arber N. Current and future clinical strategies in colon cancer prevention and the emerging role of chemoprevention. Curr Pharm Des. 2007;13:2274-82.

225. Yazici C, Wolf PG, Liu T-W, Vermillion K, Carroll T, Mutlu E, Tussing-Humphreys L, Braunschweig C, Xicola RM, Jung B. Abstract B31: Sulfidogenic bacteria are an important dietdriven exposure promoting colorectal cancer in African Americans. AACR; 2017.

226. Cao X, Ding 1, Xie Z-Z, Yang Y, Whiteman M, Moore PK, Bian J-S. A review of hydrogen sulfide synthesis, metabolism and measurement: Is modulation of hydrogen sulfide a novel therapeutic for cancer? Antioxidants and Redox Signaling. 2018.

227. Hale VL, Jeraldo P, Mundy M, Yao J, Keeney G, Scott N, Cheek EH, Davidson J, Green M, Martinez C. Synthesis of multi-omic data and community metabolic models reveals insights into the role of hydrogen sulfide in colon cancer. Methods. 2018.

228. Phillips CM. Nutrigenetics and metabolic disease: current status and implications for personalised nutrition. Nutrients. 2013 Jan 10;5:32-57.

229. Ordovas JM, Corella D. Nutritional genomics. Annu Rev Genomics Hum Genet. 2004;5:71-118.

230. Stover PJ. Nutritional genomics. Physiol Genomics. 2004 Jan 15;16:161-5.

231. Elliott R, Ong TJ. Nutritional genomics. BMJ. 2002 Jun 15;324:1438-42.

232. Trujillo E, Davis C, Milner J. Nutrigenomics, proteomics, metabolomics, and the practice of dietetics. J Am Diet Assoc. 2006 Mar;106:403-13.

233. Breton J, Tennoune N, Lucas N, Francois M, Legrand R, Jacquemot J, Goichon A, Guerin C, Peltier J, et al. Gut Commensal E. coli Proteins Activate Host Satiety Pathways following Nutrient-Induced Bacterial Growth. Cell Metab. 2016 Feb 9;23:324-34.

234. Khan MJ, Gerasimidis K, Edwards CA, Shaikh MG. Role of Gut Microbiota in the Aetiology of Obesity: Proposed Mechanisms and Review of the Literature. J Obes. 2016;2016:7353642.

235. Fuentes CT, Schellekens H, Hoevenaars N, Ross P, Roy B, Stanton C, Dinan TG, Cryan JF. Identification of Novel Probiotics to Modify Appetite and Satiety Directly Targeting the Ghrelin Receptor. The FASEB Journal. 2016;30:717.2.

236. Burcelin R. When gut fermentation controls satiety: A PYY story. Molecular Metabolism. 2017;6:10-1.

237. Fetissov SO. Role of the gut microbiota in host appetite control: bacterial growth to animal feeding behaviour. Nat Rev Endocrinol. 2017 Jan;13:11-25. 
238. Liang Q, Chiu J, Chen Y, Huang Y, Higashimori A, Fang J, Brim H, Ashktorab H, Ng $\mathrm{SC}$, et al. Fecal Bacteria Act as Novel Biomarkers for Noninvasive Diagnosis of Colorectal Cancer. Clin Cancer Res. 2017 Apr 15;23:2061-70.

239. Siegel RL, Miller KD, Fedewa SA, Ahnen DJ, Meester RGS, Barzi A, Jemal A. Colorectal cancer statistics, 2017. CA Cancer J Clin. 2017 May 6;67:177-93.

240. Pimentel-Nunes P, Goncalves N, Boal-Carvalho I, Afonso L, Lopes P, RonconAlbuquerque R, Jr., Soares JB, Cardoso E, Henrique R, et al. Decreased Toll-interacting protein and peroxisome proliferator-activated receptor gamma are associated with increased expression of Toll-like receptors in colon carcinogenesis. J Clin Pathol. 2012 Apr;65:302-8.

241. Shamsuddin AM. 2 - Normal and Pathological Anatomy of the Large Intestine. Colon Cancer Cells: Academic Press; 1990;15-40.

242. Chang WCL, Chapkin RS, Lupton JR. Fish oil blocks azoxymethane-induced rat colon tumorigenesis by increasing cell differentiation and apoptosis rather than decreasing cell proliferation. J Nutr. 1998 Mar;128:491-7.

243. Davidson LA, Nguyen DV, Hokanson RM, Callaway ES, Isett RB, Turner ND, Dougherty ER, Wang N, Lupton JR, et al. Chemopreventive n-3 polyunsaturated fatty acids reprogram genetic signatures during colon cancer initiation and progression in the rat. Cancer Res. 2004 Sep 15;64:6797-804.

244. Cho Y, Kim H, Turner ND, Mann JC, Wei J, Taddeo SS, Davidson LA, Wang N, Vannucci M, et al. A chemoprotective fish oil- and pectin-containing diet temporally alters gene expression profiles in exfoliated rat colonocytes throughout oncogenesis. J Nutr. 2011 Jun;141:1029-35.

245. Ritchie LE, Taddeo SS, Weeks BR, Carroll RJ, Dykes L, Rooney LW, Turner ND. Impact of Novel Sorghum Bran Diets on DSS-Induced Colitis. Nutrients. 2017 Mar 27;9:330.

246. Fierer N, Hamady M, Lauber CL, Knight R. The influence of sex, handedness, and washing on the diversity of hand surface bacteria. Proc Natl Acad Sci U S A. 2008 Nov 18;105:17994-9.

247. Liu Z, Lozupone C, Hamady M, Bushman FD, Knight R. Short pyrosequencing reads suffice for accurate microbial community analysis. Nucleic Acids Res. 2007;35:e120.

248. Wu GD, Lewis JD, Hoffmann C, Chen YY, Knight R, Bittinger K, Hwang J, Chen J, Berkowsky R, et al. Sampling and pyrosequencing methods for characterizing bacterial communities in the human gut using 16S sequence tags. BMC Microbiol. 2010 Jul 30;10:206.

249. Caporaso JG, Kuczynski J, Stombaugh J, Bittinger K, Bushman FD, Costello EK, Fierer N, Pena AG, Goodrich JK, et al. QIIME allows analysis of high-throughput community sequencing data. Nat Methods. 2010 May;7:335-6. 
250. Reeder J, Knight R. Rapid denoising of pyrosequencing amplicon data: exploiting the rank-abundance distribution. Nature methods. 2010;7:668.

251. Edgar RC. Search and clustering orders of magnitude faster than BLAST. Bioinformatics. 2010 Oct 1;26:2460-1.

252. Ohta T, Masutomi N, Tsutsui N, Sakairi T, Mitchell M, Milburn MV, Ryals JA, Beebe $\mathrm{KD}$, Guo L. Untargeted metabolomic profiling as an evaluative tool of fenofibrate-induced toxicology in Fischer 344 male rats. Toxicol Pathol. 2009 Jun;37:521-35.

253. Evans AM, DeHaven CD, Barrett T, Mitchell M, Milgram E. Integrated, nontargeted ultrahigh performance liquid chromatography/electrospray ionization tandem mass spectrometry platform for the identification and relative quantification of the small-molecule complement of biological systems. Anal Chem. 2009 Aug 15;81:6656-67.

254. DeHaven CD, Evans AM, Dai H, Lawton KA. Organization of GC/MS and LC/MS metabolomics data into chemical libraries. J Cheminform. 2010;2:9.

255. Jump RL, Polinkovsky A, Hurless K, Sitzlar B, Eckart K, Tomas M, Deshpande A, Nerandzic MM, Donskey CJ. Metabolomics analysis identifies intestinal microbiota-derived biomarkers of colonization resistance in clindamycin-treated mice. PLoS One. 2014;9:e101267.

256. Storey JD, Tibshirani R. Statistical significance for genomewide studies. Proc Natl Acad Sci U S A. 2003 Aug 5;100:9440-5.

257. Cheng L, Lai M-D. Aberrant crypt foci as microscopic precursors of colorectal cancer. World journal of gastroenterology: WJG. 2003;9:2642.

258. Takayama T, Katsuki S, Takahashi Y, Ohi M, Nojiri S, Sakamaki S, Kato J, Kogawa K, Miyake H, Niitsu Y. Aberrant crypt foci of the colon as precursors of adenoma and cancer. N Engl J Med. 1998;339:1277-84.

259. Stacewicz-Sapuntzakis M. Dried plums and their products: composition and health effects--an updated review. Crit Rev Food Sci Nutr. 2013 Jan 1;53:1277-302.

260. Husoy T, Haugen M, Murkovic M, Jobstl D, Stolen LH, Bjellaas T, Ronningborg C, Glatt H, Alexander J. Dietary exposure to 5-hydroxymethylfurfural from Norwegian food and correlations with urine metabolites of short-term exposure. Food Chem Toxicol. 2008 Dec;46:3697-702.

261. Podda R, Delli Castelli D, Digilio G, Gullino ML, Aime S. Asparagine in plums detected by CEST-MRI. Food Chem. 2015 Feb 15;169:1-4.

262. Heinonen S, Nurmi T, Liukkonen K, Poutanen K, Wahala K, Deyama T, Nishibe S, Adlercreutz $\mathrm{H}$. In vitro metabolism of plant lignans: new precursors of mammalian lignans enterolactone and enterodiol. J Agric Food Chem. 2001 Jul;49:3178-86. 
263. Collett ED, Davidson LA, Fan YY, Lupton JR, Chapkin RS. n-6 and n-3 polyunsaturated fatty acids differentially modulate oncogenic Ras activation in colonocytes. Am J Physiol Cell Physiol. 2001 May;280:C1066-75.

264. Kramer A, Green J, Pollard J, Jr., Tugendreich S. Causal analysis approaches in Ingenuity Pathway Analysis. Bioinformatics. 2014 Feb 15;30:523-30.

265. Yang L, Pei Z. Bacteria, inflammation, and colon cancer. World J Gastroenterol. 2006 Nov 14;12:6741-6.

266. Takahashi M, Wakabayashi K. Gene mutations and altered gene expression in azoxymethane-induced colon carcinogenesis in rodents. Cancer Sci. 2004 Jun;95:475-80.

267. Kim DO, Lee KW, Chun OK, Leer HJ, Lee CY. Antiproliferative activity of polyphenolics in plums. Food Sci Biotechnol. 2003 Aug;12:399-402.

268. Chun OK, Kim DO, Lee CY. Superoxide radical scavenging activity of the major polyphenols in fresh plums. J Agric Food Chem. 2003 Dec 31;51:8067-72.

269. Yang L, Seki E. Toll-like receptors in liver fibrosis: cellular crosstalk and mechanisms. Front Physiol. 2012 May 22;3:138.

270. Fung KY, Cosgrove L, Lockett T, Head R, Topping DL. A review of the potential mechanisms for the lowering of colorectal oncogenesis by butyrate. Br J Nutr. 2012 Sep;108:820-31.

271. Wei H, Dong L, Wang T, Zhang M, Hua W, Zhang C, Pang X, Chen M, Su M, et al. Structural shifts of gut microbiota as surrogate endpoints for monitoring host health changes induced by carcinogen exposure. FEMS Microbiol Ecol. 2010 Sep;73:577-86.

272. Kaakoush NO. Insights into the Role of Erysipelotrichaceae in the Human Host. Front Cell Infect Microbiol. 2015 Nov 20;5:84.

273. Cai WJ, Wang MJ, Ju LH, Wang C, Zhu YC. Hydrogen sulfide induces human colon cancer cell proliferation: role of Akt, ERK and p21. Cell Biol Int. 2010 Apr 14;34:565-72.

274. Yatsunenko T, Rey FE, Manary MJ, Trehan I, Dominguez-Bello MG, Contreras M, Magris M, Hidalgo G, Baldassano RN, et al. Human gut microbiome viewed across age and geography. Nature. 2012 May 9;486:222-7.

275. Zhao L, Chen J, Su J, Li L, Hu S, Li B, Zhang X, Xu Z, Chen T. In vitro antioxidant and antiproliferative activities of 5-hydroxymethylfurfural. J Agric Food Chem. 2013 Nov 6;61:10604-11.

276. Kowalski S, Lukasiewicz M, Duda-Chodak A, Zięć G. 5-Hydroxymethyl-2-Furfural (HMF) - Heat-Induced Formation, Occurrence in Food and Biotransformation - a Review. Polish Journal of Food and Nutrition Sciences. 2013;207. 
277. Hedelin M, Lof M, Sandin S, Adami HO, Weiderpass E. Prospective Study of Dietary Phytoestrogen Intake and the Risk of Colorectal Cancer. Nutr Cancer. 2016 Apr 2;68:388-95.

278. Jiang R, Botma A, Rudolph A, Husing A, Chang-Claude J. Phyto-oestrogens and colorectal cancer risk: a systematic review and dose-response meta-analysis of observational studies. Br J Nutr. 2016 Dec;116:2115-28.

279. Triff K, Kim E, Chapkin RS. Chemoprotective epigenetic mechanisms in a colorectal cancer model: Modulation by n-3 PUFA in combination with fermentable fiber. Curr Pharmacol Rep. 2015 Feb;1:11-20.

280. Chapkin RS, Hong MY, Fan YY, Davidson LA, Sanders LM, Henderson CE, Barhoumi R, Burghardt RC, Turner ND, Lupton JR. Dietary n-3 PUFA alter colonocyte mitochondrial membrane composition and function. Lipids. 2002 Feb;37:193-9.

281. Kim E, Davidson LA, Patil BS, Jayaprakasha GK, Callaway ES, Turner ND, Chapkin RS. Effects of chemoprotective diets on crypt adult stem cells: the cells of origin of colon cancer (819.1). The FASEB Journal. 2014 April 1;28.

282. Chapkin RS, Davidson LA, Ly L, Weeks BR, Lupton JR, McMurray DN.

Immunomodulatory effects of (n-3) fatty acids: putative link to inflammation and colon cancer. J Nutr. 2007 Jan;137:200S-4S.

283. Turk HF, Kolar SS, Fan YY, Cozby CA, Lupton JR, Chapkin RS. Linoleic acid and butyrate synergize to increase Bcl-2 levels in colonocytes. Int J Cancer. 2011 Jan 1;128:63-71.

284. Chapkin RS, Seo J, McMurray DN, Lupton JR. Mechanisms by which docosahexaenoic acid and related fatty acids reduce colon cancer risk and inflammatory disorders of the intestine. Chem Phys Lipids. 2008 May;153:14-23.

285. Chapkin RS, DeClercq V, Kim E, Fuentes NR, Fan YY. Mechanisms by Which Pleiotropic Amphiphilic n-3 PUFA Reduce Colon Cancer Risk. Curr Colorectal Cancer Rep. 2014 Dec 1;10:442-52.

286. Davidson LA, Brown RE, Chang WC, Morris JS, Wang N, Carroll RJ, Turner ND, Lupton JR, Chapkin RS. Morphodensitometric analysis of protein kinase $\mathrm{C}$ beta(II) expression in rat colon: modulation by diet and relation to in situ cell proliferation and apoptosis.

Carcinogenesis. 2000 Aug;21:1513-9.

287. Turner ND, Zhang J, Davidson LA, Lupton JR, Chapkin RS. Oncogenic ras alters sensitivity of mouse colonocytes to butyrate and fatty acid mediated growth arrest and apoptosis. Cancer Lett. 2002 Dec 1;186:29-35.

288. Fan YY, Zhan Y, Aukema HM, Davidson LA, Zhou L, Callaway E, Tian Y, Weeks BR, Lupton JR, et al. Proapoptotic effects of dietary (n-3) fatty acids are enhanced in colonocytes of manganese-dependent superoxide dismutase knockout mice. J Nutr. 2009 Jul;139:1328-32. 
289. Steen EJ, Kang Y, Bokinsky G, Hu Z, Schirmer A, McClure A, Del Cardayre SB, Keasling JD. Microbial production of fatty-acid-derived fuels and chemicals from plant biomass. Nature. 2010 Jan 28;463:559-62.

290. Certik M, Shimizu S. Biosynthesis and regulation of microbial polyunsaturated fatty acid production. J Biosci Bioeng. 1999;87:1-14.

291. Zargar A, Bailey CB, Haushalter RW, Eiben CB, Katz L, Keasling JD. Leveraging microbial biosynthetic pathways for the generation of 'drop-in'biofuels. Curr Opin Biotechnol. 2017;45:156-63.

292. Salonen A, de Vos WM, Palva A. Gastrointestinal microbiota in irritable bowel syndrome: present state and perspectives. Microbiology. 2010 Nov;156:3205-15.

293. David LA, Maurice CF, Carmody RN, Gootenberg DB, Button JE, Wolfe BE, Ling AV, Devlin AS, Varma Y, et al. Diet rapidly and reproducibly alters the human gut microbiome. Nature. 2014 Jan 23;505:559-63.

294. Thompson AL, Monteagudo-Mera A, Cadenas MB, Lampl ML, Azcarate-Peril MA. Milk- and solid-feeding practices and daycare attendance are associated with differences in bacterial diversity, predominant communities, and metabolic and immune function of the infant gut microbiome. Frontiers in cellular and infection microbiology. 2015;5:3.

295. Allali I, Delgado S, Marron PI, Astudillo A, Yeh JJ, Ghazal H, Amzazi S, Keku T, Azcarate-Peril MA. Gut microbiome compositional and functional differences between tumor and non-tumor adjacent tissues from cohorts from the US and Spain. Gut Microbes. 2015 Apr $15 ; 6: 161-72$.

296. Devine AA, Gonzalez A, Speck KE, Knight R, Helmrath MA, Lund PK, Azcarate Peril MA. Impact of ileocecal resection and concomitant antibiotics on the microbiome of the murine jejunum and colon. PLoS One. 2013;8(8): e73140.

297. Allali I, Arnold JW, Roach J, Cadenas MB, Butz N, Hassan HM, Koci M, Ballou A, Mendoza M, et al. A comparison of sequencing platforms and bioinformatics pipelines for compositional analysis of the gut microbiome. BMC Microbiol. 2017 Sep 13;17:194.

298. Monteagudo-Mera A, Arthur JC, Jobin C, Keku TO, Bruno Barcena JM, Azcarate-Peril MA. High purity galacto-oligosaccharides enhance specific Bifidobacterium species and their metabolic activity in the mouse gut microbiome. Beneficial microbes. 2016;3:1-18.

299. Price MN, Dehal PS, Arkin AP. FastTree 2--approximately maximum-likelihood trees for large alignments. PLoS One. 2010;5:e9490.

300. Lozupone C, Hamady M, Knight R. UniFrac--an online tool for comparing microbial community diversity in a phylogenetic context. BMC Bioinformatics. 2006 Aug 7;7:371. 
301. Tuohy KM, Conterno L, Gasperotti M, Viola R. Up-regulating the human intestinal microbiome using whole plant foods, polyphenols, and/or fiber. J Agric Food Chem. 2012 Sep 12;60:8776-82.

302. Manach C, Williamson G, Morand C, Scalbert A, Rémésy C. Bioavailability and bioefficacy of polyphenols in humans. I. Review of 97 bioavailability studies. The American journal of clinical nutrition. 2005;81:230S-42S.

303. Derrien M, Vaughan EE, Plugge CM, de Vos WM. Akkermansia muciniphila gen. nov., sp. nov., a human intestinal mucin-degrading bacterium. Int J Syst Evol Microbiol. 2004 Sep;54:1469-76.

304. Derrien M, Belzer C, de Vos WM. Akkermansia muciniphila and its role in regulating host functions. Microb Pathog. 2017 May;106:171-81.

305. Roopchand DE, Carmody RN, Kuhn P, Moskal K, Rojas-Silva P, Turnbaugh PJ, Raskin I. Dietary Polyphenols Promote Growth of the Gut Bacterium Akkermansia muciniphila and Attenuate High-Fat Diet-Induced Metabolic Syndrome. Diabetes. 2015 Aug;64:2847-58.

306. Gerassy-Vainberg S, Blatt A, Danin-Poleg Y, Gershovich K, Sabo E, Nevelsky A, Daniel S, Dahan A, Ziv O, et al. Radiation induces proinflammatory dysbiosis: transmission of inflammatory susceptibility by host cytokine induction. Gut. 2018 Jan;67:97-107.

307. Anhê FF, Roy D, Pilon G, Dudonné S, Matamoros S, Varin TV, Garofalo C, Moine Q, Desjardins Y, Levy E. A polyphenol-rich cranberry extract protects from diet-induced obesity, insulin resistance and intestinal inflammation in association with increased Akkermansia spp. population in the gut microbiota of mice. Gut. 2015;64:872-83.

308. Shin NR, Lee JC, Lee HY, Kim MS, Whon TW, Lee MS, Bae JW. An increase in the Akkermansia spp. population induced by metformin treatment improves glucose homeostasis in diet-induced obese mice. Gut. 2014 May;63:727-35.

309. Zhang Z, Wu X, Cao S, Wang L, Wang D, Yang H, Feng Y, Wang S, Li L. Caffeic acid ameliorates colitis in association with increased Akkermansia population in the gut microbiota of mice. Oncotarget. 2016 May 31;7:31790-9.

310. Alam A, Leoni G, Quiros M, Wu H, Desai C, Nishio H, Jones RM, Nusrat A, Neish AS. The microenvironment of injured murine gut elicits a local pro-restitutive microbiota. Nat Microbiol. 2016 Jan 27;1:15021.

311. Seidel DV, Hicks KK, Taddeo SS, Azcarate-Peril MA, Carroll RJ, Turner ND. Dried plums modify fecal short chain fatty acid concentrations and gene expression in a rat model of colon carcinogenesis. The FASEB Journal. 2016;30:147.3-.3.

312. Seidel DV, Taddeo SS, Azcarate-Peril MA, Carroll RJ, Turner ND. Dried Plums Modify the Colon Luminal Metabolome in a Rat Model of Colon Carcinogenesis. The FASEB Journal. 2017;31:590.5. 
313. Datta K, Suman S, Kallakury BV, Fornace AJ, Jr. Exposure to heavy ion radiation induces persistent oxidative stress in mouse intestine. PLoS One. 2012;7:e42224.

314. Arjmandi BH, Khalil DA, Lucas EA, Georgis A, Stoecker BJ, Hardin C, Payton ME, Wild RA. Dried plums improve indices of bone formation in postmenopausal women. J Womens Health Gend Based Med. 2002 Jan-Feb;11:61-8.

315. Deyhim F, Stoecker BJ, Brusewitz GH, Devareddy L, Arjmandi BH. Dried plum reverses bone loss in an osteopenic rat model of osteoporosis. Menopause. 2005 Nov-Dec;12:755-62.

316. Franklin M, Bu SY, Lerner MR, Lancaster EA, Bellmer D, Marlow D, Lightfoot SA, Arjmandi BH, Brackett DJ, et al. Dried plum prevents bone loss in a male osteoporosis model via IGF-I and the RANK pathway. Bone. 2006 Dec;39:1331-42.

317. Halloran BP, Wronski TJ, VonHerzen DC, Chu V, Xia X, Pingel JE, Williams AA, Smith BJ. Dietary Dried Plum Increases Bone Mass in Adult and Aged Male Mice-3. The Journal of nutrition. 2010;140:1781-7.

318. Hooshmand S, Kern M, Metti D, Shamloufard P, Chai SC, Johnson SA, Payton ME, Arjmandi BH. The effect of two doses of dried plum on bone density and bone biomarkers in osteopenic postmenopausal women: a randomized, controlled trial. Osteoporos Int. 2016 Jul;27:2271-9.

319. Rendina E, Lim YF, Marlow D, Wang Y, Clarke SL, Kuvibidila S, Lucas EA, Smith BJ. Dietary supplementation with dried plum prevents ovariectomy-induced bone loss while modulating the immune response in C57BL/6J mice. J Nutr Biochem. 2012;23:60-8.

320. Schreurs A-S, Shirazi Y, Shahnazari M, S Alwood J, A Truong T, G T Tahimic C, Limoli C, Turner N, Halloran B, Globus R. Dried plum diet protects from bone loss caused by ionizing radiation; 2016.

321. Fertil B, Malaise EP. Intrinsic radiosensitivity of human cell lines is correlated with radioresponsiveness of human tumors: Analysis of 101 published survival curves. International Journal of Radiation Oncology*Biology*Physics. 1985 Sep 1;11:1699-707.

322. Baumann M, Krause M, Hill R. Exploring the role of cancer stem cells in radioresistance. Nat Rev Cancer. 2008 Jul;8:545-54.

323. Chen MF, Lin CT, Chen WC, Yang CT, Chen CC, Liao SK, Liu JM, Lu CH, Lee KD. The sensitivity of human mesenchymal stem cells to ionizing radiation. Int J Radiat Oncol Biol Phys. 2006 Sep 1;66:244-53.

324. Deschavanne PJ, Fertil B. A review of human cell radiosensitivity in vitro. Int J Radiat Oncol Biol Phys. 1996 Jan 1;34:251-66.

325. T. James P, Rigby N, Leach R. The obesity epidemic, metabolic syndrome and future prevention strategies. Eur J Cardiovasc Prev Rehabil. 2004;11:3-8. 
326. Giovannucci E. Metabolic syndrome, hyperinsulinemia, and colon cancer: a review. Am J Clin Nutr. 2007 Sep;86:s836-42.

327. Cowey S, Hardy RW. The metabolic syndrome: A high-risk state for cancer? Am J Pathol. 2006 Nov;169:1505-22.

328. Cani PD, Osto M, Geurts L, Everard A. Involvement of gut microbiota in the development of low-grade inflammation and type 2 diabetes associated with obesity. Gut Microbes. 2012 Jul;3:279-88.

329. Heilbronn LK, Campbell LV. Adipose tissue macrophages, low grade inflammation and insulin resistance in human obesity. Curr Pharm Des. 2008;14:1225-30.

330. Maachi M, Pieroni L, Bruckert E, Jardel C, Fellahi S, Hainque B, Capeau J, Bastard J. Systemic low-grade inflammation is related to both circulating and adipose tissue TNF $\alpha$, leptin and IL-6 levels in obese women. Int J Obes. 2004;28:993.

331. Kau AL, Ahern PP, Griffin NW, Goodman AL, Gordon JI. Human nutrition, the gut microbiome and the immune system. Nature. 2011 Jun 15;474:327-36.

332. Gao Z, Yin J, Zhang J, Ward RE, Martin RJ, Lefevre M, Cefalu WT, Ye J. Butyrate improves insulin sensitivity and increases energy expenditure in mice. Diabetes. 2009 Jul;58:1509-17.

333. Turnbaugh PJ, Ley RE, Mahowald MA, Magrini V, Mardis ER, Gordon JI. An obesityassociated gut microbiome with increased capacity for energy harvest. Nature. 2006 Dec 21;444:1027-31.

334. Le Chatelier E, Nielsen T, Qin J, Prifti E, Hildebrand F, Falony G, Almeida M, Arumugam M, Batto JM, et al. Richness of human gut microbiome correlates with metabolic markers. Nature. 2013 Aug 29;500:541-6.

335. Vrieze A, Van Nood E, Holleman F, Salojarvi J, Kootte RS, Bartelsman JF, DallingaThie GM, Ackermans MT, Serlie MJ, et al. Transfer of intestinal microbiota from lean donors increases insulin sensitivity in individuals with metabolic syndrome. Gastroenterology. 2012 Oct;143:913-6.

336. Ridaura VK, Faith JJ, Rey FE, Cheng J, Duncan AE, Kau AL, Griffin NW, Lombard V, Henrissat B, et al. Gut microbiota from twins discordant for obesity modulate metabolism in mice. Science. 2013 Sep 6;341:1241214.

337. Ley RE, Turnbaugh PJ, Klein S, Gordon JI. Microbial ecology: human gut microbes associated with obesity. Nature. 2006 Dec 21;444:1022-3.

338. Tjalsma H, Boleij A, Marchesi JR, Dutilh BE. A bacterial driver-passenger model for colorectal cancer: beyond the usual suspects. Nat Rev Microbiol. 2012 Jun 25;10:575-82. 
339. Murphy EF, Cotter PD, Healy S, Marques TM, O'Sullivan O, Fouhy F, Clarke SF, O'Toole PW, Quigley EM, et al. Composition and energy harvesting capacity of the gut microbiota: relationship to diet, obesity and time in mouse models. Gut. 2010 Dec;59:1635-42.

340. Rajilic-Stojanovic M, Biagi E, Heilig HG, Kajander K, Kekkonen RA, Tims S, de Vos WM. Global and deep molecular analysis of microbiota signatures in fecal samples from patients with irritable bowel syndrome. Gastroenterology. 2011 Nov;141:1792-801.

341. Hagerman AE, Riedl KM, Jones GA, Sovik KN, Ritchard NT, Hartzfeld PW, Riechel TL. High Molecular Weight Plant Polyphenolics (Tannins) as Biological Antioxidants. J Agric Food Chem. 1998 May;46:1887-92.

342. Serrano J, Puupponen-Pimia R, Dauer A, Aura AM, Saura-Calixto F. Tannins: current knowledge of food sources, intake, bioavailability and biological effects. Mol Nutr Food Res. 2009 Sep;53 Suppl 2:S310-29.

343. Bhat TK, Singh B, Sharma OP. Microbial degradation of tannins--a current perspective. Biodegradation. 1998;9:343-57.

344. Heider J, Fuchs G. Microbial anaerobic aromatic metabolism. Anaerobe. 1997 Feb;3:122.

345. Smith AH, Mackie RI. Effect of condensed tannins on bacterial diversity and metabolic activity in the rat gastrointestinal tract. Appl Environ Microbiol. 2004 Feb;70:1104-15.

346. Lemlioglu-Austin D, Turner ND, McDonough CM, Rooney LW. Effects of sorghum [Sorghum bicolor (L.) Moench] crude extracts on starch digestibility, Estimated Glycemic Index (EGI), and Resistant Starch (Rs) contents of porridges. Molecules. 2012 Sep 17;17:11124-38.

347. Austin DL, Turner ND, McDonough CM, Rooney LW. Effects of brans from specialty sorghum varieties on in vitro starch digestibility of soft and hard sorghum endosperm porridges. Cereal Chem. 2012;89:190-7.

348. Ritchie LE, Carroll R, Weeks B, Rooney L, Turner N. Novel sorghum brans containing bioactive compounds alter the production of microbial secondary metabolites in response to a DSS-induced chronic inflammatory state. The FASEB Journal. 2012;26:823.36.

349. Center NC. NDS-R software. Minneapolis: Regents of the University of Minnesota. 2003.

350. Martinez I, Lattimer JM, Hubach KL, Case JA, Yang J, Weber CG, Louk JA, Rose DJ, Kyureghian G, et al. Gut microbiome composition is linked to whole grain-induced immunological improvements. ISME J. 2013 Feb;7:269-80.

351. Martinez I, Kim J, Duffy PR, Schlegel VL, Walter J. Resistant starches types 2 and 4 have differential effects on the composition of the fecal microbiota in human subjects. PLoS One. 2010 Nov 29;5:e15046. 
352. Sridharan GV, Choi K, Klemashevich C, Wu C, Prabakaran D, Pan LB, Steinmeyer S, Mueller C, Yousofshahi M, et al. Prediction and quantification of bioactive microbiota metabolites in the mouse gut. Nat Commun. 2014 Nov 20;5:5492.

353. Bajad SU, Lu W, Kimball EH, Yuan J, Peterson C, Rabinowitz JD. Separation and quantitation of water soluble cellular metabolites by hydrophilic interaction chromatographytandem mass spectrometry. J Chromatogr A. 2006 Aug 25;1125:76-88.

354. Gonthier MP, Remesy C, Scalbert A, Cheynier V, Souquet JM, Poutanen K, Aura AM. Microbial metabolism of caffeic acid and its esters chlorogenic and caftaric acids by human faecal microbiota in vitro. Biomed Pharmacother. 2006 Nov;60:536-40.

355. Gonthier MP, Verny MA, Besson C, Remesy C, Scalbert A. Chlorogenic acid bioavailability largely depends on its metabolism by the gut microflora in rats. J Nutr. 2003 Jun;133:1853-9.

356. Wikoff WR, Anfora AT, Liu J, Schultz PG, Lesley SA, Peters EC, Siuzdak G. Metabolomics analysis reveals large effects of gut microflora on mammalian blood metabolites. Proc Natl Acad Sci U S A. 2009 Mar 10;106:3698-703.

357. Moss CW, Lambert M, Goldsmith D. Production of hydrocinnamic acid by clostridia. Appl Microbiol. 1970;19:375-8.

358. Hoskins JA, Holliday SB, Greenway AM. The metabolism of cinnamic acid by healthy and phenylketonuric adults: a kinetic study. Biomed Mass Spectrom. 1984 Jun;11:296-300.

359. Pekkinen J, Rosa NN, Savolainen OI, Keski-Rahkonen P, Mykkanen H, Poutanen K, Micard V, Hanhineva K. Disintegration of wheat aleurone structure has an impact on the bioavailability of phenolic compounds and other phytochemicals as evidenced by altered urinary metabolite profile of diet-induced obese mice. Nutr Metab (Lond). 2014 Jan 2;11:1.

360. Obrenovich ME, Tima M, Polinkovsky A, Zhang R, Emancipator SN, Donskey CJ. Targeted Metabolomics Analysis Identifies Intestinal Microbiota-Derived Urinary Biomarkers of Colonization Resistance in Antibiotic-Treated Mice. Antimicrob Agents Chemother. 2017 Aug;61.

361. Tang WH, Hazen SL. The contributory role of gut microbiota in cardiovascular disease. J Clin Invest. 2014 Oct;124:4204-11.

362. Fujii H, Nakai K, Fukagawa M. Role of oxidative stress and indoxyl sulfate in progression of cardiovascular disease in chronic kidney disease. Ther Apher Dial. 2011 Apr;15:125-8.

363. Moreno-Navarrete JM, Ortega F, Serino M, Luche E, Waget A, Pardo G, Salvador J, Ricart W, Fruhbeck G, et al. Circulating lipopolysaccharide-binding protein (LBP) as a marker of obesity-related insulin resistance. Int J Obes (Lond). 2012 Nov;36:1442-9. 
364. Selma MV, Espin JC, Tomas-Barberan FA. Interaction between phenolics and gut microbiota: role in human health. J Agric Food Chem. 2009 Aug 12;57:6485-501.

365. Jacobs DM, Spiesser L, Garnier M, de Roo N, van Dorsten F, Hollebrands B, van Velzen E, Draijer R, van Duynhoven J. SPE-NMR metabolite sub-profiling of urine. Anal Bioanal Chem. 2012 Nov;404:2349-61.

366. Brown GK, Stokke O, Jellum E. Chromatographic profile of high boiling point organic acids in human urine. J Chromatogr. 1978 Mar 1;145:177-84.

367. Moss CW, Lambert MA, Goldsmith DJ. Production of hydrocinnamic acid by clostridia. Appl Microbiol. 1970 Feb;19:375-8.

368. Bo S, Gambino R, Durazzo M, Guidi S, Tiozzo E, Ghione F, Gentile L, Cassader M, Pagano GF. Associations between gamma-glutamyl transferase, metabolic abnormalities and inflammation in healthy subjects from a population-based cohort: a possible implication for oxidative stress. World J Gastroenterol. 2005 Dec 7;11:7109-17.

369. Fornaciari I, Fierabracci V, Corti A, Aziz Elawadi H, Lorenzini E, Emdin M, Paolicchi A, Franzini M. Gamma-glutamyltransferase fractions in human plasma and bile: characteristic and biogenesis. PLoS One. 2014;9:e88532.

370. Lee DS, Evans JC, Robins SJ, Wilson PW, Albano I, Fox CS, Wang TJ, Benjamin EJ, D'Agostino RB, Vasan RS. Gamma glutamyl transferase and metabolic syndrome,

cardiovascular disease, and mortality risk: the Framingham Heart Study. Arterioscler Thromb Vasc Biol. 2007 Jan;27:127-33.

371. Mason JE, Starke RD, Van Kirk JE. Gamma-glutamyl transferase: a novel cardiovascular risk biomarker. Prev Cardiol. 2010 Winter;13:36-41.

372. Whitfield-Cargile CM, Cohen ND, Chapkin RS, Weeks BR, Davidson LA, Goldsby JS, Hunt CL, Steinmeyer SH, Menon R, et al. The microbiota-derived metabolite indole decreases mucosal inflammation and injury in a murine model of NSAID enteropathy. Gut Microbes. 2016 May 3;7:246-61.

373. Diaz-Diaz CJ, Ronnekleiv-Kelly SM, Nukaya M, Geiger PG, Balbo S, Dator R, Megna BW, Carney PR, Bradfield CA, Kennedy GD. The Aryl Hydrocarbon Receptor is a Repressor of Inflammation-associated Colorectal Tumorigenesis in Mouse. Ann Surg. 2016 Sep;264:429-36.

374. Jin UH, Lee SO, Sridharan G, Lee K, Davidson LA, Jayaraman A, Chapkin RS, Alaniz $\mathrm{R}$, Safe S. Microbiome-derived tryptophan metabolites and their aryl hydrocarbon receptordependent agonist and antagonist activities. Mol Pharmacol. 2014 May;85:777-88.

375. Manach C, Scalbert A, Morand C, Remesy C, Jimenez L. Polyphenols: food sources and bioavailability. Am J Clin Nutr. 2004 May;79:727-47. 
376. Zhao X, Fritsche J, Wang J, Chen J, Rittig K, Schmitt-Kopplin P, Fritsche A, Haring HU, Schleicher ED, et al. Metabonomic fingerprints of fasting plasma and spot urine reveal human pre-diabetic metabolic traits. Metabolomics. 2010 Sep;6:362-74.

377. Wu G, Fang YZ, Yang S, Lupton JR, Turner ND. Glutathione metabolism and its implications for health. J Nutr. 2004 Mar;134:489-92.

378. Wu GD, Chen J, Hoffmann C, Bittinger K, Chen YY, Keilbaugh SA, Bewtra M, Knights D, Walters WA, et al. Linking long-term dietary patterns with gut microbial enterotypes. Science. 2011 Oct 7;334:105-8.

379. Meehan CJ, Beiko RG. A phylogenomic view of ecological specialization in the Lachnospiraceae, a family of digestive tract-associated bacteria. Genome Biol Evol. 2014 Mar;6:703-13.

380. Dworkin M, Falkow S. Prokaryotes: Delta and Epsilon Subclasses, Deeply Rooting Bacteria: a Handbook on the Biology of Bacteria: Springer; 2006.

381. Cho I, Yamanishi S, Cox L, Methe BA, Zavadil J, Li K, Gao Z, Mahana D, Raju K, et al. Antibiotics in early life alter the murine colonic microbiome and adiposity. Nature. 2012 Aug 30;488:621-6.

382. Duncan SH, Barcenilla A, Stewart CS, Pryde SE, Flint HJ. Acetate utilization and butyryl coenzyme A (CoA):acetate-CoA transferase in butyrate-producing bacteria from the human large intestine. Appl Environ Microbiol. 2002 Oct;68:5186-90.

383. Zeng H, Ishaq SL, Zhao FQ, Wright AG. Colonic inflammation accompanies an increase of beta-catenin signaling and Lachnospiraceae/Streptococcaceae bacteria in the hind gut of highfat diet-fed mice. J Nutr Biochem. 2016 Sep;35:30-6.

384. Kameyama K, Itoh K. Intestinal colonization by a Lachnospiraceae bacterium contributes to the development of diabetes in obese mice. Microbes Environ. 2014;29:427-30.

385. Martin R, Miquel S, Benevides L, Bridonneau C, Robert V, Hudault S, Chain F, Berteau $\mathrm{O}$, Azevedo V, et al. Functional Characterization of Novel Faecalibacterium prausnitzii Strains Isolated from Healthy Volunteers: A Step Forward in the Use of F. prausnitzii as a NextGeneration Probiotic. Front Microbiol. 2017;8:1226.

386. Miquel S, Martin R, Rossi O, Bermudez-Humaran LG, Chatel JM, Sokol H, Thomas M, Wells JM, Langella P. Faecalibacterium prausnitzii and human intestinal health. Curr Opin Microbiol. 2013 Jun;16:255-61.

387. Sokol H, Pigneur B, Watterlot L, Lakhdari O, Bermudez-Humaran LG, Gratadoux JJ, Blugeon S, Bridonneau C, Furet JP, et al. Faecalibacterium prausnitzii is an anti-inflammatory commensal bacterium identified by gut microbiota analysis of Crohn disease patients. Proc Natl Acad Sci U S A. 2008 Oct 28;105:16731-6. 
388. Sokol H, Seksik P, Furet JP, Firmesse O, Nion-Larmurier I, Beaugerie L, Cosnes J, Corthier G, Marteau P, Dore J. Low counts of Faecalibacterium prausnitzii in colitis microbiota. Inflamm Bowel Dis. 2009 Aug;15:1183-9.

389. Varela E, Manichanh C, Gallart M, Torrejon A, Borruel N, Casellas F, Guarner F, Antolin M. Colonisation by Faecalibacterium prausnitzii and maintenance of clinical remission in patients with ulcerative colitis. Aliment Pharmacol Ther. 2013 Jul;38:151-61.

390. Van Immerseel F, Ducatelle R, De Vos M, Boon N, Van De Wiele T, Verbeke K, Rutgeerts P, Sas B, Louis P, Flint HJ. Butyric acid-producing anaerobic bacteria as a novel probiotic treatment approach for inflammatory bowel disease. J Med Microbiol. 2010;59:141-3.

391. Benus RF, van der Werf TS, Welling GW, Judd PA, Taylor MA, Harmsen HJ, Whelan K. Association between Faecalibacterium prausnitzii and dietary fibre in colonic fermentation in healthy human subjects. Br J Nutr. 2010;104:693-700.

392. Ouwerkerk JP, Aalvink S, Belzer C, de Vos WM. Akkermansia glycaniphila sp. nov., an anaerobic mucin-degrading bacterium isolated from reticulated python faeces. Int J Syst Evol Microbiol. 2016 Nov;66:4614-20.

393. Anhê FF, Pilon G, Roy D, Desjardins Y, Levy E, Marette A. Triggering Akkermansia with dietary polyphenols: A new weapon to combat the metabolic syndrome? Gut microbes. 2016;7:146-53.

394. Picolli da Silva L, de Lourdes Santorio Ciocca M. Total, insoluble and soluble dietary fiber values measured by enzymatic-gravimetric method in cereal grains. J Food Compost Anal. 2005 Feb 1;18:113-20.

395. Pimpao RC, Ventura MR, Ferreira RB, Williamson G, Santos CN. Phenolic sulfates as new and highly abundant metabolites in human plasma after ingestion of a mixed berry fruit puree. Br J Nutr. 2015 Feb 14;113:454-63.

396. Fang C, Kim H, Barnes RC, Talcott ST, Mertens-Talcott SU. Obesity-Associated Diseases Biomarkers Are Differently Modulated in Lean and Obese Individuals and Inversely Correlated to Plasma Polyphenolic Metabolites After 6 Weeks of Mango (Mangifera indica L.) Consumption. Mol Nutr Food Res. 2018 May 24;62:e1800129.

397. Rechner AR, Kuhnle G, Hu H, Roedig-Penman A, van den Braak MH, Moore KP, RiceEvans CA. The metabolism of dietary polyphenols and the relevance to circulating levels of conjugated metabolites. Free Radic Res. 2002 Nov;36:1229-41.

398. Rechner AR, Kuhnle G, Bremner P, Hubbard GP, Moore KP, Rice-Evans CA. The metabolic fate of dietary polyphenols in humans. Free Radic Biol Med. 2002 Jul 15;33:220-35.

399. Gonthier M-P, Rios LY, Verny M-A, Rémésy C, Scalbert A. Novel liquid chromatography-electrospray ionization mass spectrometry method for the quantification in 
human urine of microbial aromatic acid metabolites derived from dietary polyphenols. Journal of Chromatography B. 2003 Jun 15;789:247-55.

400. Whitfield JB. Gamma glutamyl transferase. Crit Rev Clin Lab Sci. 2001 Aug;38:263355.

401. Lim JS, Yang JH, Chun BY, Kam S, Jacobs DR, Jr., Lee DH. Is serum gammaglutamyltransferase inversely associated with serum antioxidants as a marker of oxidative stress? Free Radic Biol Med. 2004 Oct 1;37:1018-23.

402. Pompella A, Visvikis A, Paolicchi A, De Tata V, Casini AF. The changing faces of glutathione, a cellular protagonist. Biochem Pharmacol. 2003 Oct 15;66:1499-503.

403. Garcia TC, Steffey MA, Zwingenberger AL, Daniel L, Stover SM. CT-derived indices of canine osteosarcoma-affected antebrachial strength. Vet Surg. 2017 May;46:549-58.

404. Abdi H, Williams LJ. Principal component analysis. Wiley interdisciplinary reviews: computational statistics. 2010;2:433-59.

405. Bro R, Smilde AK. Principal component analysis. Analytical Methods. 2014;6:2812-31.

406. Thompson B. Canonical correlation analysis. Encyclopedia of statistics in behavioral science. 2005.

407. Koenig JE, Spor A, Scalfone N, Fricker AD, Stombaugh J, Knight R, Angenent LT, Ley RE. Succession of microbial consortia in the developing infant gut microbiome. Proc Natl Acad Sci U S A. 2011 Mar 15;108 Suppl 1:4578-85.

408. Cani PD, Everard A. Talking microbes: when gut bacteria interact with diet and host organs. Mol Nutr Food Res. 2016;60:58-66.

409. Schroeder BO, Backhed F. Signals from the gut microbiota to distant organs in physiology and disease. Nat Med. 2016 Oct 6;22:1079-89.

410. Sommer F, Backhed F. Know your neighbor: Microbiota and host epithelial cells interact locally to control intestinal function and physiology. Bioessays. 2016 May;38:455-64.

411. Marchesi JR, Adams DH, Fava F, Hermes GD, Hirschfield GM, Hold G, Quraishi MN, Kinross J, Smidt H, et al. The gut microbiota and host health: a new clinical frontier. Gut. 2016 Feb;65:330-9.

412. Ochoa-Repáraz J, Kirby TO, Kasper LH. The gut microbiome and multiple sclerosis. Cold Spring Harb Perspect Med. 2018;8:a029017.

413. Kitsios GD, Morowitz MJ, Dickson RP, Huffnagle GB, McVerry BJ, Morris A. Dysbiosis in the intensive care unit: Microbiome science coming to the bedside. J Crit Care. 2017 Apr;38:84-91. 
414. Dinan TG, Borre YE, Cryan JF. Genomics of schizophrenia: time to consider the gut microbiome? Mol Psychiatry. 2014 Dec;19:1252-7.

415. Bi Y, Qin N, Yang R. Human microbiota: a neglected "organ" in precision medicine. Infectious Diseases and Translational Medicine. 2015;1:63-72.

416. Clarke G, Stilling RM, Kennedy PJ, Stanton C, Cryan JF, Dinan TG. Minireview: Gut microbiota: the neglected endocrine organ. Mol Endocrinol. 2014 Aug;28:1221-38.

417. Marteau P, Doré J. Gut microbiota: An organ all its own. 2017.

418. Wroblewski LE, Shen L, Ogden S, Romero-Gallo J, Lapierre LA, Israel DA, Turner JR, Peek RM, Jr. Helicobacter pylori dysregulation of gastric epithelial tight junctions by ureasemediated myosin II activation. Gastroenterology. 2009 Jan;136:236-46.

419. Roper J, Francois F, Shue PL, Mourad MS, Pei Z, Olivares de Perez AZ, Perez-Perez GI, Tseng CH, Blaser MJ. Leptin and ghrelin in relation to Helicobacter pylori status in adult males. J Clin Endocrinol Metab. 2008 Jun;93:2350-7.

420. El-Omar EM, Oien K, El-Nujumi A, Gillen D, Wirz A, Dahill S, Williams C, Ardill JE, McColl KE. Helicobacter pylori infection and chronic gastric acid hyposecretion.

Gastroenterology. 1997 Jul;113:15-24.

421. Blaser MJ. Disappearing microbiota: Helicobacter pylori protection against esophageal adenocarcinoma. Cancer Prev Res (Phila). 2008 Oct;1:308-11.

422. Amieva MR, Vogelmann R, Covacci A, Tompkins LS, Nelson WJ, Falkow S. Disruption of the epithelial apical-junctional complex by Helicobacter pylori CagA. Science. 2003 May 30;300:1430-4.

423. Courtay C, Oster T, Michelet F, Visvikis A, Diederich M, Wellman M, Siest G. Gammaglutamyltransferase: nucleotide sequence of the human pancreatic cDNA. Evidence for a ubiquitous gamma-glutamyltransferase polypeptide in human tissues. Biochem Pharmacol. 1992 Jun 23;43:2527-33.

424. Buckel W, Barker HA. Two pathways of glutamate fermentation by anaerobic bacteria. J Bacteriol. 1974 Mar;117:1248-60.

425. Liu Q, Zhang J, Wei XX, Ouyang SP, Wu Q, Chen GQ. Microbial production of L glutamate and L -glutamine by recombinant Corynebacterium glutamicum harboring Vitreoscilla hemoglobin gene vgb. Appl Microbiol Biotechnol. 2008 Jan;77:1297-304.

426. Sleiman SF, Henry J, Al-Haddad R, El Hayek L, Abou Haidar E, Stringer T, Ulja D, Karuppagounder SS, Holson EB, et al. Exercise promotes the expression of brain derived neurotrophic factor (BDNF) through the action of the ketone body beta-hydroxybutyrate. Elife. 2016 Jun 2;5:e15092. 
427. Stipanuk MH, Caudill MA. Biochemical, Physiological, and Molecular Aspects of Human Nutrition-E-Book: Elsevier health sciences; 2013.

428. Cavaleri F, Bashar E. Potential Synergies of beta-Hydroxybutyrate and Butyrate on the Modulation of Metabolism, Inflammation, Cognition, and General Health. J Nutr Metab. 2018;2018:7195760.

429. Hu S, Dong TS, Dalal SR, Wu F, Bissonnette M, Kwon JH, Chang EB. The microbederived short chain fatty acid butyrate targets miRNA-dependent p21 gene expression in human colon cancer. PLoS One. 2011 Jan 20;6:e16221.

430. Leonel AJ, Alvarez-Leite JI. Butyrate: implications for intestinal function. Curr Opin Clin Nutr Metab Care. 2012 Sep;15:474-9.

431. Everard A, Belzer C, Geurts L, Ouwerkerk JP, Druart C, Bindels LB, Guiot Y, Derrien M, Muccioli GG, et al. Cross-talk between Akkermansia muciniphila and intestinal epithelium controls diet-induced obesity. Proc Natl Acad Sci U S A. 2013 May 28;110:9066-71.

432. Lees HJ, Swann JR, Wilson ID, Nicholson JK, Holmes E. Hippurate: the natural history of a mammalian-microbial cometabolite. J Proteome Res. 2013;12:1527-46.

433. Scheline RR, Midtvedt T. Absence of dehydroxylation of caffeic acid in germ-free rats. Experientia. 1970 Oct 15;26:1068-9.

434. Peppercorn MA, Goldman P. Caffeic acid metabolism by gnotobiotic rats and their intestinal bacteria. Proceedings of the National Academy of Sciences. 1972;69:1413-5.

435. Siegel RL, Miller KD, Jemal A. Cancer Statistics, 2017. CA Cancer J Clin. 2017 Jan;67:7-30.

436. Scalbert A, Manach C, Morand C, Remesy C, Jimenez L. Dietary polyphenols and the prevention of diseases. Crit Rev Food Sci Nutr. 2005 Jun 1;45:287-306.

437. Matsunaga K, Katayama M, Sakata K, Kuno T, Yoshida K, Yamada Y, Hirose Y, Yoshimi N, Mori H. Inhibitory Effects of Chlorogenic Acid on Azoxymethane-induced Colon Carcinogenesis in Male F344 Rats. Asian Pac J Cancer Prev. 2002;3:163-6.

438. Vannini F, Kashfi K, Nath N. The dual role of iNOS in cancer. Redox Biol. 2015 Dec;6:334-43.

439. Karin M, Greten FR. NF-kappaB: linking inflammation and immunity to cancer development and progression. Nat Rev Immunol. 2005 Oct;5:749-59.

440. Scharlau D, Borowicki A, Habermann N, Hofmann T, Klenow S, Miene C, Munjal U, Stein K, Glei M. Mechanisms of primary cancer prevention by butyrate and other products formed during gut flora-mediated fermentation of dietary fibre. Mutat Res. 2009 Jul;682:39-53. 
441. Woo SS, Jiang J, Gill BS, Paterson AH, Wing RA. Construction and characterization of a bacterial artificial chromosome library of Sorghum bicolor. Nucleic Acids Res. 1994 Nov 25;22:4922-31.

442. de Morais Cardoso L, Pinheiro SS, Martino HS, Pinheiro-Sant'Ana HM. Sorghum (Sorghum bicolor L.): Nutrients, bioactive compounds, and potential impact on human health. Crit Rev Food Sci Nutr. 2017 Jan 22;57:372-90.

443. Awika JM, Rooney LW, Waniska RD. Properties of 3-deoxyanthocyanins from sorghum. J Agric Food Chem. 2004 Jul 14;52:4388-94.

444. Iwei LGU, Ouse SUEH, Ooney LLR, Rior ROLP. Sorghum Bran in the Diet Dose Dependently Increased the Excretion of Catechins and Microbial-Derived Phenolic Acids in Female Rats. 2007:5326-34.

445. Ritchie LE. Diet, Disease State, and the Space Environment Modify the Intestinal Microbiota and Mucosal Environment via Microbiota-directed Alterations in Colonocyte Signalling. Doctoral dissertation, Texas A\&M University. 2013.

446. Yassour M, Lim MY, Yun HS, Tickle TL, Sung J, Song YM, Lee K, Franzosa EA, Morgan XC, et al. Sub-clinical detection of gut microbial biomarkers of obesity and type 2 diabetes. Genome Med. 2016 Feb 17;8:17.

447. Arimochi H, Kinouchi T, Kataoka K, Kuwahara T, Ohnishi Y. Effect of intestinal bacteria on formation of azoxymethane-induced aberrant crypt foci in the rat colon. Biochem Biophys Res Commun. 1997 Sep 29;238:753-7.

448. Schroeder A, Mueller O, Stocker S, Salowsky R, Leiber M, Gassmann M, Lightfoot S, Menzel W, Granzow M, Ragg T. The RIN: an RNA integrity number for assigning integrity values to RNA measurements. BMC Mol Biol. 2006 Jan 31;7:3. 


\section{APPENDIX A}

\section{TABLES AND FIGURES OF RESULTS}

Table A-1. Diet ingredients $(\mathrm{g} / \mathrm{kg})$ and estimated nutrient composition of both diets $(\mathrm{g} / 100 \mathrm{~g})$.

\begin{tabular}{|c|c|c|}
\hline Ingredient & $\begin{array}{l}\text { Basal } \\
\text { (g/kg) }\end{array}$ & $\begin{array}{l}\text { Plum }^{1} \\
(\mathrm{~g} / \mathrm{kg})\end{array}$ \\
\hline Dextrose $^{2}$ & 484.8 & 436.8 \\
\hline Casein $^{2}$ & 221.6 & 220.4 \\
\hline DL-methionine $^{2}$ & 3.4 & 3.4 \\
\hline AIN-76 mineral mix ${ }^{2}$ & 38.8 & 38.8 \\
\hline AIN-76A vitamin mix $^{2}$ & 11.1 & 11.1 \\
\hline Choline bitartrate $^{2}$ & 2.2 & 2.2 \\
\hline Pectin $^{2}$ & 71.9 & 65.4 \\
\hline Cellulose $^{2}$ & 17.2 & 15.6 \\
\hline Corn oil $^{3}$ & 149.0 & 148.9 \\
\hline Dried plum puree $^{4}$ & 0.0 & 92.3 \\
\hline Nutrient composition & \multicolumn{2}{|c|}{$(\mathrm{g} / 100 \mathrm{~g})$} \\
\hline Total Carbohydrate & \multicolumn{2}{|c|}{57.06} \\
\hline Dietary Fiber & \multicolumn{2}{|c|}{6.00} \\
\hline Soluble & \multicolumn{2}{|c|}{4.27} \\
\hline Insoluble & \multicolumn{2}{|c|}{1.73} \\
\hline Sugar & \multicolumn{2}{|c|}{51.06} \\
\hline Protein & \multicolumn{2}{|c|}{22.35} \\
\hline Lipid & \multicolumn{2}{|c|}{15.00} \\
\hline
\end{tabular}

${ }^{1}$ Dried plum puree contains water and micronutrients, which causes the Plum $(\mathrm{g} / \mathrm{kg})$ column to sum to $1.034 \mathrm{~kg}$. ${ }^{2}$ Manufactured by Harlan (CA.160190, CA.160030, CA10850, CA.170915.PWD, CA.40077.PWD, CA.30190, CA.160390, SS-62)

${ }^{3}$ Provided by Dyets, Inc. (401150)

${ }^{4}$ Provided by Sunsweet Growers, Inc. (7045017316) 
Table A-2. Assay ID for selected gene targets in Chapter II.

\begin{tabular}{|l|l|l|l|r|}
\hline Gene Symbol(s) & Gene Alias(es) & Assay ID & Species & $\begin{array}{l}\text { Amplicon } \\
\text { Length }\end{array}$ \\
\hline Tlr2 & - & Rn02133647_s1 & Rat & 109 \\
\hline Tlr4 & - & Rn00569848_m1 & Rat & 127 \\
\hline Tollip & - & Rn01479669_m1 & Rat & 64 \\
\hline $18 s$ rRNA & - & Hs99999901_s1 & Human & 0 \\
\hline Slc5a8 & RGD1564146 & Rn01503812_m1 & Rat & 60 \\
\hline Slc16a1 & MCT1;RATMCT1;RNMCT1 & Rn00562332_m1 & Rat & 77 \\
\hline Nfkb1 & Rn01399583_m1 & Rat & 63 \\
\hline Nfkbia & RL/IF-1 & Rn01473657_g1 & Rat & 71 \\
\hline Ptgs2 & COX-2;Cox2 & Rn01483828_m1 & Rat & 112 \\
\hline Acads & Scad & Rn00574634_m1 & Rat & 87 \\
\hline Acadm & MCAD & Rn00566390_m1 & Rat & 73 \\
\hline Gapdh & BARS-38;Gapd & Rn01775763_g1 & Rat & 174 \\
\hline Muc2 & HH-Muc & Rn01498196_g1 & Rat & 77 \\
\hline Nos2 & Nos2a;iNos & Rn01475265_m1 & Rat & 77 \\
\hline Muc4 & ASGP-1;Psmc & Rn01451270_g1 & Rat & 83 \\
\hline Muc5ac & - & & & \\
\hline
\end{tabular}


Table A-3. Percent abundances of select genera organized by proximal and distal colon and injection treatment.

\begin{tabular}{|c|c|c|c|c|c|c|}
\hline AOM Effects & \multicolumn{3}{|c|}{ Proximal Colon } & \multicolumn{3}{|c|}{ Distal Colon } \\
\hline Genus & $\begin{array}{l}\text { Saline Injected } \\
\text { (\% abundance) }\end{array}$ & $\begin{array}{l}\text { AOM Injected } \\
\text { (\% abundance) })\end{array}$ & $P$-value & $\begin{array}{l}\text { Saline Injected } \\
\text { (\% abundance) }\end{array}$ & $\begin{array}{l}\text { AOM Injected } \\
\text { (\% abundance) }\end{array}$ & $P$-value \\
\hline Corynebacterium & $6.75 \mathrm{E}-04 \pm 7.60 \mathrm{E}-04$ & $3.12 \mathrm{E}-03 \pm 6.34 \mathrm{E}-04$ & 0.0172 & $1.86 \mathrm{E}-03 \pm 8.63 \mathrm{E}-04$ & $2.52 \mathrm{E}-03 \pm 7.06 \mathrm{E}-04$ & 0.5558 \\
\hline Rikenellaceae; $g$ & $2.30 \mathrm{E}-01 \pm 5.94 \mathrm{E}-02$ & $2.70 \mathrm{E}-01 \pm 4.96 \mathrm{E}-02$ & 0.6003 & $2.44 \mathrm{E}-01 \pm 5.02 \mathrm{E}-02$ & $3.94 \mathrm{E}-01 \pm 4.11 \mathrm{E}-02$ & 0.0255 \\
\hline Odoribacter & $1.76 \mathrm{E}-04 \pm 5.24 \mathrm{E}-04$ & $7.35 \mathrm{E}-04 \pm 4.38 \mathrm{E}-04$ & 0.4166 & $2.46 \mathrm{E}-04 \pm 9.36 \mathrm{E}-05$ & $0.00 \pm 7.66 \mathrm{E}-05$ & 0.0477 \\
\hline Elusimicrobiaceae; $g$ & $2.75 \mathrm{E}-02 \pm 1.26 \mathrm{E}-02$ & $6.25 \mathrm{E}-02 \pm 1.05 \mathrm{E}-02$ & 0.0387 & $3.84 \mathrm{E}-02 \pm 1.47 \mathrm{E}-02$ & $6.15 \mathrm{E}-02 \pm 1.20 \mathrm{E}-02$ & 0.2295 \\
\hline Lachnospiraceae; Other & $1.68 \pm 7.18 \mathrm{E}-01$ & $3.53 \pm 5.99 \mathrm{E}-01$ & 0.0536 & $1.20 \pm 8.45 \mathrm{E}-01$ & $4.12 \pm 6.91 \mathrm{E}-01$ & 0.0103 \\
\hline Ruminococcaceae; Other & $4.67 \mathrm{E}-01 \pm 9.32 \mathrm{E}-02$ & $3.50 \mathrm{E}-01 \pm 7.78 \mathrm{E}-02$ & 0.3410 & $5.24 \mathrm{E}-01 \pm 8.66 \mathrm{E}-02$ & $2.77 \mathrm{E}-01 \pm 7.09 \mathrm{E}-02$ & 0.0324 \\
\hline$O P B 54 ; f ; g$ & $3.92 \mathrm{E}-04 \pm 1.48 \mathrm{E}-04$ & $0.00 \pm 1.24 \mathrm{E}-04$ & 0.0482 & $1.45 \mathrm{E}-04 \pm 1.09 \mathrm{E}-04$ & $6.80 \mathrm{E}-05 \pm 8.92 \mathrm{E}-05$ & 0.5873 \\
\hline Ralstonia & $1.24 \mathrm{E}-04 \pm 1.08 \mathrm{E}-04$ & $7.18 \mathrm{E}-05 \pm 9.01 \mathrm{E}-05$ & 0.7131 & $3.64 \mathrm{E}-04 \pm 1.24 \mathrm{E}-04$ & $3.73 \mathrm{E}-05 \pm 1.02 \mathrm{E}-04$ & 0.0475 \\
\hline
\end{tabular}

Diet groups were combined for this main effect analysis. Proximal and distal colon data were analyzed separately. Data are LSMEANS \pm SEM. 
Table A-4. Percent abundances of select genera organized by proximal and distal colon and experimental diet.

\begin{tabular}{|c|c|c|c|c|c|c|}
\hline Diet Effects & \multicolumn{3}{|c|}{ Proximal Colon } & \multicolumn{3}{|c|}{ Distal Colon } \\
\hline Genus & $\begin{array}{c}\text { Basal Diet } \\
\text { (\% abundance) }\end{array}$ & $\begin{array}{c}\text { Plum Diet } \\
(\% \text { abundance })\end{array}$ & $P$-value & $\begin{array}{c}\text { Basal Diet } \\
\text { (\% abundance) }\end{array}$ & $\begin{array}{c}\text { Plum Diet } \\
(\% \text { abundance })\end{array}$ & $P$-value \\
\hline CoriobacteriaceaeG & $3.60 \mathrm{E}-03 \pm 3.65 \mathrm{E}-02$ & $4.54 \mathrm{E}-01 \pm 3.49 \mathrm{E}-02$ & $<.0001$ & $4.32 \mathrm{E}-03 \pm 4.64 \mathrm{E}-02$ & $4.22 \mathrm{E}-01 \pm 4.52 \mathrm{E}-02$ & $<.0001$ \\
\hline Adlercreutzia & $9.02 \mathrm{E}-02 \pm 9.91 \mathrm{E}-03$ & $6.53 \mathrm{E}-02 \pm 9.50 \mathrm{E}-03$ & 0.0759 & $1.22 \mathrm{E}-01 \pm 1.20 \mathrm{E}-02$ & $7.59 \mathrm{E}-02 \pm 1.17 \mathrm{E}-02$ & 0.0092 \\
\hline Bacteroides & $2.01 \mathrm{E}+01 \pm 1.66 \mathrm{E}+00$ & $1.99 \mathrm{E}+01 \pm 1.59 \mathrm{E}+00$ & 0.9393 & $1.58 \mathrm{E}+01 \pm 1.92 \mathrm{E}+00$ & $2.59 \mathrm{E}+01 \pm 1.87 \mathrm{E}+00$ & 0.0005 \\
\hline Parabacteroides & $6.46 \mathrm{E}-01 \pm 1.01 \mathrm{E}-01$ & $9.33 \mathrm{E}-01 \pm 9.67 \mathrm{E}-02$ & 0.0459 & $6.79 \mathrm{E}-01 \pm 1.98 \mathrm{E}-01$ & $1.57 \pm 1.93 \mathrm{E}-01$ & 0.0024 \\
\hline Porphyromonas & n.d. & n.d. & -- & $0.00 \pm 2.56 \mathrm{E}-04$ & $7.44 \mathrm{E}-04 \pm 2.49 \mathrm{E}-04$ & 0.043 \\
\hline RikenellaceaeG & $2.45 \mathrm{E}-01 \pm 5.59 \mathrm{E}-02$ & $2.55 \mathrm{E}-01 \pm 5.36 \mathrm{E}-02$ & 0.8912 & $1.96 \mathrm{E}-01 \pm 4.64 \mathrm{E}-02$ & $4.42 \mathrm{E}-01 \pm 4.52 \mathrm{E}-02$ & 0.0004 \\
\hline$S 247 G$ & $3.89 \pm 3.71 \mathrm{E}-01$ & $4.79 \pm 3.55 \mathrm{E}-01$ & 0.0895 & $3.63 \pm 3.81 \mathrm{E}-01$ & $4.85 \pm 3.71 \mathrm{E}-01$ & 0.0268 \\
\hline BarnesiellaceaeG & $1.87 \mathrm{E}-01 \pm 5.22 \mathrm{E}-02$ & $2.53 \mathrm{E}-01 \pm 5.01 \mathrm{E}-02$ & & $1.42 \mathrm{E}-01 \pm 6.46 \mathrm{E}-02$ & $3.91 \mathrm{E}-01 \pm 6.29 \mathrm{E}-02$ & 0.0082 \\
\hline Butyricimonas & $4.51 \mathrm{E}-02 \pm 1.06 \mathrm{E}-02$ & $7.16 \mathrm{E}-02 \pm 1.02 \mathrm{E}-02$ & 0.0797 & $4.11 \mathrm{E}-02 \pm 1.96 \mathrm{E}-02$ & $1.29 \mathrm{E}-01 \pm 1.91 \mathrm{E}-02$ & 0.0025 \\
\hline Paraprevotella & $6.55 \mathrm{E}-02 \pm 3.02 \mathrm{E}-02$ & $3.48 \mathrm{E}-01 \pm 2.89 \mathrm{E}-02$ & $<.0001$ & $3.40 \mathrm{E}-02 \pm 3.76 \mathrm{E}-02$ & $4.20 \mathrm{E}-01 \pm 3.66 \mathrm{E}-02$ & $<.0001$ \\
\hline Mucispirillum & $3.17 \mathrm{E}-02 \pm 6.72 \mathrm{E}-03$ & $9.60 \mathrm{E}-04 \pm 6.44 \mathrm{E}-03$ & 0.0019 & $4.24 \mathrm{E}-02 \pm 1.33 \mathrm{E}-02$ & $0.00 \pm 1.29 \mathrm{E}-02$ & 0.0266 \\
\hline Lactobacillus & $1.58 \mathrm{E}+01 \pm 1.69$ & $1.04 \mathrm{E}+01 \pm 1.62$ & 0.0264 & $1.54 \mathrm{E}+01 \pm 1.67$ & $7.34 \pm 1.63$ & 0.0011 \\
\hline ClostridialesFG & $9.25 \pm 1.15$ & $9.81 \pm 1.10$ & 0.7288 & $1.11 \mathrm{E}+01 \pm 8.54 \mathrm{E}-01$ & $8.63 \pm 8.31 \mathrm{E}-01$ & 0.0429 \\
\hline ChristensenellaceaeG & $1.22 \mathrm{E}-02 \pm 3.74 \mathrm{E}-03$ & $2.47 \mathrm{E}-02 \pm 3.58 \mathrm{E}-03$ & 0.0195 & $1.21 \mathrm{E}-02 \pm 3.10 \mathrm{E}-03$ & $2.70 \mathrm{E}-02 \pm 3.02 \mathrm{E}-03$ & 0.0013 \\
\hline ClostridiaceaeG & $3.24 \pm 3.23 \mathrm{E}-01$ & $1.67 \pm 3.09 \mathrm{E}-01$ & 0.001 & $2.13 \pm 1.93 \mathrm{E}-01$ & $9.71 \mathrm{E}-01 \pm 1.87 \mathrm{E}-01$ & $<.0001$ \\
\hline LachnospiraceaeOther & $9.20 \mathrm{E}-01 \pm 6.75 \mathrm{E}-01$ & $4.29 \pm 6.47 \mathrm{E}-01$ & 0.0008 & 7.04E-01 $\pm 7.82 \mathrm{E}-01$ & $4.62 \pm 7.61 \mathrm{E}-01$ & 0.0008 \\
\hline LachnospiraceaeG & $2.02 \pm 3.82 \mathrm{E}-01$ & $3.71 \pm 3.66 \mathrm{E}-01$ & 0.0026 & $1.96 \pm 3.98 \mathrm{E}-01$ & $3.25 \pm 3.88 \mathrm{E}-01$ & 0.0255 \\
\hline Dorea & $5.97 \mathrm{E}-02 \pm 1.68 \mathrm{E}-01$ & $6.63 \mathrm{E}-01 \pm 1.61 \mathrm{E}-01$ & 0.0128 & $1.98 \mathrm{E}-01 \pm 9.24 \mathrm{E}-02$ & $3.87 \mathrm{E}-01 \pm 9.00 \mathrm{E}-02$ & 0.1502 \\
\hline Peptococcaceaerc44G & $3.52 \mathrm{E}-01 \pm 5.34 \mathrm{E}-02$ & $4.68 \mathrm{E}-01 \pm 5.12 \mathrm{E}-02$ & 0.1238 & $2.01 \mathrm{E}-01 \pm 5.12 \mathrm{E}-02$ & $3.62 \mathrm{E}-01 \pm 4.99 \mathrm{E}-02$ & 0.0299 \\
\hline ErysipelotrichaceaeOther & $9.00 \mathrm{E}-04 \pm 4.25 \mathrm{E}-04$ & $9.61 \mathrm{E}-04 \pm 4.07 \mathrm{E}-04$ & 0.9178 & $1.09 \mathrm{E}-03 \pm 2.56 \mathrm{E}-04$ & $1.34 \mathrm{E}-04 \pm 2.49 \mathrm{E}-04$ & 0.0104 \\
\hline ErysipelotrichaceaeG & $1.11 \mathrm{E}-02 \pm 3.77 \mathrm{E}-03$ & $5.89 \mathrm{E}-03 \pm 3.62 \mathrm{E}-03$ & 0.3251 & $2.43 \mathrm{E}-02 \pm 6.14 \mathrm{E}-03$ & $3.59 \mathrm{E}-03 \pm 5.98 \mathrm{E}-03$ & 0.0198 \\
\hline Allobaculum & $1.46 \mathrm{E}+01 \pm 2.25$ & $9.74 \pm 2.16$ & 0.1227 & $1.63 \mathrm{E}+01 \pm 2.34$ & $8.49 \pm 2.28$ & 0.021 \\
\hline Coprobacillus & $6.20 \mathrm{E}-04 \pm 2.08 \mathrm{E}-03$ & $7.26 \mathrm{E}-03 \pm 2.00 \mathrm{E}-03$ & 0.0262 & $1.50 \mathrm{E}-03 \pm 3.31 \mathrm{E}-03$ & $1.16 \mathrm{E}-02 \pm 3.22 \mathrm{E}-03$ & 0.0342 \\
\hline Holdemania & $3.73 \mathrm{E}-04 \pm 4.73 \mathrm{E}-03$ & $1.48 \mathrm{E}-02 \pm 4.53 \mathrm{E}-03$ & 0.0332 & $4.01 \mathrm{E}-03 \pm 5.09 \mathrm{E}-03$ & $1.89 \mathrm{E}-02 \pm 4.95 \mathrm{E}-03$ & 0.0416 \\
\hline DesulfovibrionaceaeG & $2.98 \mathrm{E}-02 \pm 6.09 \mathrm{E}-03$ & $4.43 \mathrm{E}-02 \pm 5.83 \mathrm{E}-03$ & 0.093 & $2.15 \mathrm{E}-02 \pm 6.89 \mathrm{E}-03$ & $5.65 \mathrm{E}-02 \pm 6.71 \mathrm{E}-03$ & 0.0007 \\
\hline
\end{tabular}

Injection groups were combined for this main effect analysis. Proximal and distal colon data were analyzed separately. Data are LSMEANS \pm SEM. 
Table A-5. Significantly altered metabolites in plasma of human subjects consuming sumac sorghum.

\begin{tabular}{|l|l|l|l|l|l|}
\hline Metabolite & Transform & Estimate & Std. Err. & $\boldsymbol{P}$-value & $\boldsymbol{Q}$-value \\
\hline Gamma-glutamylvaline & original & -0.46956 & 0.06848 & 0 & 0 \\
\hline Threonate & original & -0.40669 & 0.07337 & $1.00 \mathrm{E}-05$ & 0.00177 \\
\hline Catechol sulfate & log & 0.37786 & 0.07154 & $3.00 \mathrm{E}-05$ & 0.00354 \\
\hline Gamma-glutamylisoleucine* & original & -0.37044 & 0.07652 & $8.00 \mathrm{E}-05$ & 0.00708 \\
\hline Gamma-glutamylmethionine & original & -0.222 & 0.05215 & 0.00032 & 0.01888 \\
\hline HWESASXX* & $\log$ & 0.65837 & 0.1546 & 0.00032 & 0.01888 \\
\hline 3-hydroxyhippurate & original & 1.03108 & 0.24635 & 0.00038 & 0.01922 \\
\hline Cinnamoylglycine & log & 0.38459 & 0.09533 & 0.00055 & 0.02434 \\
\hline Cysteine & original & -0.24734 & 0.06412 & 0.00085 & 0.03009 \\
\hline Glutamine & original & 0.05121 & 0.01323 & 0.00083 & 0.03009 \\
\hline 3-phenylpropionate (hydrocinnamate) & log & 0.32909 & 0.09755 & 0.00274 & 0.08290 \\
\hline
\end{tabular}

$P$ - and $Q$-values, estimates, and standard errors are statistics of the treatment effect (i.e., the average of the response to low and high levels of sumac sorghum).

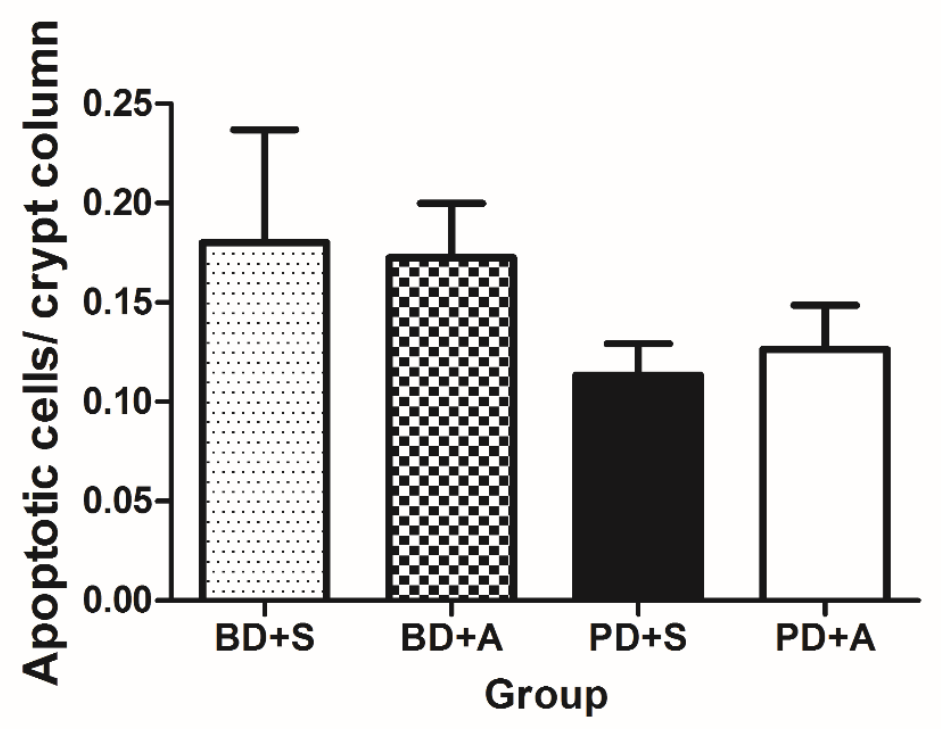

Figure A-1 Apoptotic cells/crypt column in rats fed a basal diet (BD) or dried plum diet (DP) and exposed to saline $(+\mathrm{S})$ or AOM $(+\mathrm{A})$. No significant differences were observed between any group. 


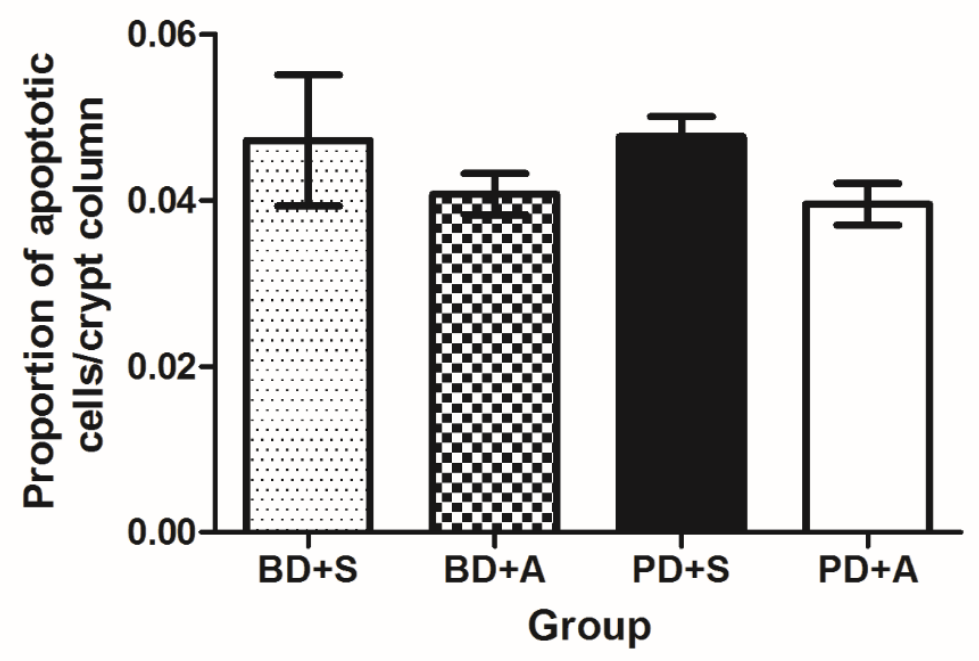

Figure A-2 Proportion of apoptotic cells/crypt column in rats fed a basal diet (BD) or dried plum diet (DP) and exposed to saline $(+\mathrm{S})$ or AOM $(+\mathrm{A})$. No significant differences were observed between any group. 


\section{APPENDIX B}

\section{EXPERIMENTAL PROTOCOLS}

\section{Chapter II Animals and Diets}

Fifty-one male Sprague-Dawley rats $(21 \mathrm{~d}$ old $)$ were stratified by body weight and assigned to one of 2 experimental diets (basal diet $n=25$, plum diet $n=26$ ). The plum diet was a slightly modified basal diet containing a dried plum puree incorporated at a level that would provide $5 \%$ of the total calories in the diet. The contribution of lipid, protein, and carbohydrate, as well as the ratio and amount of soluble to insoluble fiber were made equivalent between the diets based on the proximate analysis of the plum puree. The calculation for the final diet formulation is seen below. The macronutrients contained in the dried plum puree used in the experiment was adjusted for in the diet by balancing the inclusion of dextrose, casein and pectin added to the diet. The diets were mixed according to the following protocol:

\begin{tabular}{|c|c|c|c|c|c|}
\hline \multirow[b]{2}{*}{ INGREDIENT } & \multirow[b]{2}{*}{$\% 100$} & \multicolumn{2}{|c|}{ Amount In Total Diet } & \multicolumn{2}{|c|}{ Amount per Diet Group (kg) } \\
\hline & & g & $\mathbf{k g}$ & 1-Basal & Plum \\
\hline Dextrose & $51.06 \%$ & 100077.60 & 100.07760 & 47.84011 & 43.07847 \\
\hline Casein & $22.35 \%$ & 43806.00 & 43.80600 & 21.86754 & 21.73326 \\
\hline DL-methionine & $0.34 \%$ & 666.40 & 0.66640 & 0.33320 & 0.33320 \\
\hline Mineral mix & $3.91 \%$ & 7663.60 & 7.66360 & 3.83180 & 3.83180 \\
\hline Vitamin mix & $1.12 \%$ & 2195.20 & 2.19520 & 1.09760 & 1.09760 \\
\hline Choline bitartrate & $0.22 \%$ & 431.20 & 0.43120 & 0.21560 & 0.21560 \\
\hline Pectin & $4.27 \%$ & 8369.20 & 8.36920 & 7.09250 & 6.45050 \\
\hline Cellulose & $1.73 \%$ & 3390.80 & 3.39080 & 1.69540 & 1.54152 \\
\hline Lipid - Corn oil & $15.00 \%$ & 29400.00 & 29.40000 & 14.70000 & 14.68179 \\
\hline Test Compounds & 0.0000 & $\mathrm{~N} / \mathrm{A}$ & $\mathrm{N} / \mathrm{A}$ & 0.00000 & 9.10548 \\
\hline \multicolumn{6}{|l|}{ Water } \\
\hline \multirow[t]{5}{*}{ Total wt } & 1.0000 & 196000.0 & 196.0000 & $\begin{array}{r}98.67375 \\
\end{array}$ & 102.06922 \\
\hline & & & & units $\mathrm{H} 2 \mathrm{O}$ & 2.667905426 \\
\hline & & & & units Ash & 0.788534505 \\
\hline & & & & & 98.61278 \\
\hline & & & Total Kcal: & 411.1306 & 411.939552 \\
\hline
\end{tabular}




\section{$\underline{\text { PLUM Diet Mixing Protocol: Preparation Checklist }}$}

$\square \quad$ Adequate diet components

$\square \quad$ Plum (thoroughly mixed)

$\square \quad$ Clean and labeled Ziploc bags for diet components

$\square \quad$ Lab personnel

$\square \quad$ Weighing scales (2): $50 \mathrm{~g}-5 \mathrm{~kg}$ and $<50 \mathrm{~g}$

$\square$ Measuring tubs and scoops (spatulas)

$\square \quad$ Stirring tool

$\square$ Labeled diet bags and outer bags

$\square$ Colored tapes

$\square \quad$ Sharpies

$\square \quad$ Aluminum foil

$\square \quad$ 5L jugs for oil (2-3)

$\square \quad$ Cart, tub for ice and ice

$\square$ Gloves, bench papers, towels, and $70 \%$ ethanol

$\square$ Diet specification sheets

$\square \quad$ Mixing bowl and mixer (2)

$\square$ Labeled specimen cups for diet sample

Note: Each mixing bowl can only contain up to $20 \mathrm{~kg}$ of diet, thus the total weight of each diet needs to be divided up into $\sim 5$ portions (referred to as bowls).

\begin{tabular}{|c|c|c|c|c|c|}
\hline Diet & Bowl 1 & Bowl 2 & Bowl 3 & Bowl 4 & Bowl 5 \\
\hline Basal & 3 tubs +1 ziplock & 3 tubs +1 ziplock & 3 tubs +1 ziplock & 3 tubs +1 ziplock & 3 tubs +1 ziplock \\
\hline Plum & 3 tubs +1 ziplock & 3 tubs +1 ziplock & 3 tubs +1 ziplock & 3 tubs +1 ziplock & 3 tubs +1 ziplock \\
\hline Total & 6 tubs +2 ziplock & 6 tubs +2 ziplock & 6 tubs +2 ziplock & 6 tubs +2 ziplock & 6 tubs +2 ziplock \\
\hline
\end{tabular}

\section{Diet mixing protocol (BASAL)}

1. Bring diet ingredients and items from previous checklist to basement from lab

2. Measure out small components (Vitamin/mineral/choline/methionine) as necessary per bowl

3. Measure out corn oil per bowl.

4. Start with Bowl 1 of a diet group. Mix by hand in large bowl diet components in the following sequence:

1. Vitamin/Mineral/Methionine/Choline

2. Pectin

3. Cellulose

4. Casein

5. Dextrose

5. Fit bowl onto electric mixer, cover, and mix dry components for 5 min at \#1 setting. 
6. Still at \#1 setting, start mixer for 10 minutes. During this time, pour oil into mix 3 separate quantities and times. To get it all out from jug, use diet to mop it up.

7. Scrape sides of bowl and blade. Mix for 5 minutes more.

8. Lower bowl, scrape sides of bowl and blade, put bowl back into position, and mix for another 10 minutes at \#2 setting.

9. Scrape diet off blade, lower and remove bowl. Take a sample from 4 locations in bowl and place into $50 \mathrm{~mL}$ conical tube w/ appropriate label.

10. Scoop diet into labeled bags and double bag. Store in freezer.

11. Repeat the above process for all diets/bowls.

12. Clean up thoroughly.

\section{Diet mixing protocol (PLUM)}

1. Bring diet ingredients and items from previous checklist to basement from lab

2. Measure out small components (Vitamin/mineral/choline/methionine) as necessary per bowl

3. Measure out corn oil per bowl.

4. Start with Bowl 1 of a diet group. Mix by hand in large container (tub) diet components in the following sequence:

1. Vitamin/Mineral/Methionine/Choline

2. Pectin

3. Cellulose

4. Casein

5. Dextrose

5. Weigh out plum ( 1 bowl) into small mixer bowl. Add corn oil (1 bowl) to plum in small mixer bowl.

6. Mix Plum/Oil for 3 minutes on power level 1 with small mixer.

7. Transfer Plum/Oil mixture into large mixing bowl.

8. Fit large mixing bowl onto electric mixer. Set timer to 10 minutes and start mixing at power level 1. Over this time, slowly add dry ingredients into Plum/Oil mixture.

9. After all dry ingredients have been added (10 minutes) lower bowl and scrape mixer and sides of bowl with spatula/gloved hand.

10. Still at \#1 setting, start mixer for another 10 minutes.

11. Scrape sides of bowl and blade. Mix for 5 minutes more at \#2 setting.

12. Scrape diet off blade, lower and remove bowl. Take a sample from 4 locations in bowl and place into $50 \mathrm{~mL}$ conical tube w/ appropriate label.

13. Scoop diet into labeled bags and double bag. Store in freezer.

14. Repeat the above process for all diets/bowls.

15. Clean up thoroughly. 
Prior to beginning the experimental diet, animals were maintained on a standard chow diet for $7 \mathrm{~d}$ in order to overcome shipping stress. After $21 \mathrm{~d}$ of experimental diets, 15 animals from the basal diet group and 16 animals from the plum diet group received an injection of azoxymethane $(15 \mathrm{mg} / \mathrm{kg}$ body weight). The remaining 10 animals from each diet group received an equivalent volume of saline. These treatments were administered a second time, $7 \mathrm{~d}$ after the initial injection. During the $48 \mathrm{hr}$ following injection, animals were stored in isolation rooms with adequate ventilation to exhaust the metabolized (exhaled) carcinogen. Saline animals were kept in a separate isolation room from AOM animals to avoid any exposure to the carcinogen. Animals were provided $2 \mathrm{~d}$ worth of food to last the duration of the isolation period. At the end of the isolation period, animals were returned to their normal husbandry room at the animal facility. After the second injection, animals continued to consume their experimental diets for 8 additional weeks until their termination.

Body weight and food intake was routinely monitored. Animals were weighed (48 h average) upon arrival, prior to beginning experimental diets, before each injection, $4 \mathrm{w}$ after the second injection, and at termination. Experimental diet intake was averaged over a $48 \mathrm{~h}$ period at experimental day 20,55 , and 82 . On experimental day 91 , animals were euthanized by $\mathrm{CO}_{2}$ asphyxiation and cervical dislocation. The colon was resected, and two $1 \mathrm{~cm}$ sections were removed from the proximal and distal colon for histological preparations. The contents of the colon were collected separately from the proximal and distal colon for microbial and metabolomic analyses. The remaining colon was cut in half longitudinally: $1 / 2$ was used for ACF analysis and the other $1 / 2$ was separated into proximal and distal segments for scraped mucosa collection (see below). 


\section{Chapter II Tissue Collection and Processing}

Tissue fixation for immunohistological processing

The two, $1 \mathrm{~cm}$ segments removed from the distal end of the colon were fixed in either a 4\% PFA (PFA solution was mixed a day before the killing. Fifty $\mathrm{ml}$ of $10 \mathrm{X}$ PBS was diluted to $400 \mathrm{ml}$ with ddH2O, and then $100 \mathrm{ml}$ of $20 \%$ PFA was added. PFA solution was refrigerated overnight.) or $70 \% \mathrm{EtOH}$ solution prior to embedding in paraffin. Tissue fixed in $70 \% \mathrm{EtOH}$ was stored at room temperature until embedded in paraffin. Tissue fixed in PFA was incubated on ice for $4 \mathrm{~h}$ in a 4\% PFA solution, then rinsed in successive washes of ice cold 50\% EtOH ( $1 \mathrm{x}$ dip, 4 x 20 minutes), $70 \%$ EtOH (4 x 20 minutes), and stored at $4{ }^{\circ} \mathrm{C}$ in $70 \% \mathrm{EtOH}$ until embedded in paraffin.

Luminal contents collection and processing

Luminal contents were expressed from the proximal and distal colon separately. Samples from each location were aliquoted into tubes for microbial sequencing and metabolomics and then placed into liquid nitrogen. At the end of the termination, samples were transferred to the $-80^{\circ} \mathrm{C}$ freezer until further processing.

\section{$\underline{\text { Scraped mucosa }}$}

After the luminal material was removed from the colon, the colon was washed twice in RNase free Phosphate-Buffered Saline (PBS) and opened lengthwise to expose the luminal surface. The opened colon was then cut in half longitudinally again to separate it into two pieces. One piece was cut into proximal and distal segments for mucosal scraping. The segments were scraped on a chilled RNase free surface, and transferred to an RNase free homogenization tube along with $300 \mu \mathrm{l}$ of Denaturation Solution (Ambion, Austin, TX) or $200 \mu \mathrm{l}$ of protein buffer (see below). Scraped mucosa for RNA analysis was homogenized in Denaturation Solution for 6- 
7 strokes and then transferred to a $2 \mathrm{ml}$ epitube for storage at $-80^{\circ} \mathrm{C}$. Scraped mucosa was placed into a homogenizer along with $200 \mu \mathrm{l}$ of buffer. Protein buffer $(10 \mathrm{ml})$ was mixed on the day of the kill, just prior to use. The protein buffer contained: $1 \mathrm{ml}$ of $500 \mathrm{mM}$ Tris- $\mathrm{HCl}$ (pH 7.2) (Sigma, T1503), $2.5 \mathrm{ml}$ of 1.0 M Sucrose (Sigma, S9378), $100 \mu \mathrm{l}$ of $200 \mathrm{mM}$ EDTA (pH 7.6) (Sigma, ED4SS), $100 \mu \mathrm{l}$ of $100 \mathrm{mM}$ EGTA (pH 7.5) (Aldrich, 23453-2), $4.793 \mathrm{ml}$ of water, $1 \mathrm{ml}$ of 10\% Triton X-100 (Sigma, T6878), $7 \mu 1$ of $\beta$-mercaptoethanol (Bio-Rad, 161-0710), $400 \mu 1$ of Protease Inhibitor (Sigma), and $100 \mu 1$ Phosphatase Inhibitor (Pierce). Mucosa and protein buffer was homogenized for at least 7 strokes (on ice) and transferred into a $2 \mathrm{ml}$ eppietube. The sample was aspirated through a 12 gauge needle twice and then incubated on ice for 30 minutes prior to centrifugation at $15,000 \mathrm{~g}$ for 20 minutes. The supernatant was transferred, and the volume was split amongst the aliquot tubes and then placed into a $-80^{\circ} \mathrm{C}$ freezer for storage.

\section{$\underline{\text { ACF fixation }}$}

The remaining longitudinal half section was laid out flat on a piece of Whatman filter paper that had been moistened with PBS. The filter paper was then folded over to 'sandwich' the colon segment and the 'sandwich' was placed into a Tupperware container filled with $70 \%$

ethanol. A glass ( $1 / 4$ inch thick) plate was placed on top of the 'sandwich' so that the colon would fix flat. After $48 \mathrm{~h}$ in the Tupperware container, each colon was removed and placed into a $50 \mathrm{ml}$ scintillation vial filled with fresh $70 \%$ ethanol, until processing for ACF measurement.

\section{Chapter II fecal collections and processing}

Fresh fecal samples were collected for SCFA analysis $3 \mathrm{w}$ post-diet start, $48 \mathrm{~h}$ post-AOM $\# 2$, and for $48 \mathrm{~h}$ prior to termination. Luminal contents collected at termination were not used for SCFA analysis. Fresh fecal samples were collected within 15 minutes of defecation, placed into 
appropriately labeled cryotubes and flash frozen in liquid nitrogen. At the end of the collection period, samples were transferred into the $-80^{\circ} \mathrm{C}$ freezer until further processing. Dry matter analysis

Fecal samples were thawed and placed into a weighed dry pan prior to drying in a $60^{\circ} \mathrm{C}$ oven for $72 \mathrm{~h}$. Samples were cooled in a vacuum desiccator for $24 \mathrm{~h}$ before being weighed. This procedure was repeated until weight became static.

$\underline{\text { Short chain fatty acid (SCFA) analysis }}$

Fresh fecal samples were collected immediately following defecation. Samples were transferred to sterile cryotubes, snap-frozen in liquid nitrogen, and stored at $-80^{\circ} \mathrm{C}$. Fecal samples were prepared for analysis by grinding in a mortar and pestle chilled by immersion in liquid nitrogen. The powdered sample $(\sim 0.30 \mathrm{~g})$ was weighed into a $1.5 \mathrm{~mL}$ centrifuge tube containing $750 \mathrm{~mL}$ of the internal standard, 2-ethylbutyric acid (Sigma-Aldrich, St. Louis, MO), in 70\% ethanol. Samples were vigorously vortexed and incubated overnight at $47^{\circ} \mathrm{C}$ to extract the SCFA. To prepare the extracted samples for gas-liquid chromatography, samples were first shaken vigorously for $20 \mathrm{~min}$ and then centrifuged at $11,500 \mathrm{~g}$ for $20 \mathrm{~min}$ at $47^{\circ} \mathrm{C}$. A $200 \mu \mathrm{l}$ aliquot of the supernatant was removed and combined with $200 \mu \mathrm{l}$ of the second internal standard, $3 \mathrm{mM}$ heptanoic acid prepared in $70 \%$ ethanol. Immediately before injection into the gas chromatograph (GC), $20 \mu \mathrm{L}$ of $10 \%$ orthophosphoric acid $\left(\mathrm{H}_{3} \mathrm{PO}_{4}\right)$ was added to the sample and vortexed. One microliter of sample containing the phosphoric acid was then injected into a Varian 3900 gas chromatograph (Walnut Creek, CA) with a splitless capillary inlet and flame ionization detector equipped with a $30 \mathrm{~m}, 0.53 \mathrm{~mm}$ internal diameter deactivated glass capillary pre-column (Supelco, Bellfonte, PA). Data were integrated and plotted with a Hewlett Packard Series II Integrator. The GC conditions used were as follows: injector temperature, $165^{\circ} \mathrm{C}$; 
detector temperature, $220^{\circ} \mathrm{C}$; column flow, $2.21 \mathrm{~mL} / \mathrm{min} \mathrm{He}$; make-up flow, $28 \mathrm{~mL} / \mathrm{min}$

nitrogen; and oven temperature, $185^{\circ} \mathrm{C}$ (gradient). Samples are concentrated on the column head at $60^{\circ} \mathrm{C}$ for $30 \mathrm{sec}$. The temperature is increased at $50^{\circ} \mathrm{C} / \mathrm{min}$ to a temperature of $145^{\circ} \mathrm{C}$ for 5 minutes. A temperature gradient of $5^{\circ} \mathrm{C} / \mathrm{min}$ is continued until a final temperature of $185^{\circ} \mathrm{C}$. After the analysis, the column is allowed to regenerate at $220^{\circ} \mathrm{C}$ for 5 minutes before the temperature is reduced for the next run. Standards and a blank were run before and after the daily sample runs to facilitate calibration and data calculations.

\section{$\underline{\text { Identification and quantification of SCFA }}$}

Short chain fatty acid standards containing 2-ETBA, heptanoic acid and $\mathrm{H}_{3} \mathrm{PO}_{4}$ are made fresh, stored at $-70^{\circ} \mathrm{C}$, and chromatographed at the beginning and end of daily sample runs. Blanks containing only internal standard solutions in $70 \%$ ethanol are also chromatographed daily. Response factors and retention times for the individual fatty acids are determined from the results of the daily standard runs and fatty acid composition of samples are calculated based on the appropriate daily standard response factor.

\section{Chapter II ACF analysis}

\section{$\underline{\text { ACF scoring protocol }}$}

\section{Staining}

1. Cover sample ID upon retrieval of sample to remain blind to sample

2. Place transparent grid sheet on microscope plate. Grids make it easier to read and score samples.

3. Remove colon from scintillation vial with forceps. Scooping is preferred over pinching so that the tissue isn't damaged.

4. Stain the colon with $0.5 \%$ methylene blue. Staining time depends on sample thickness, but should be as uniform across samples as possible. ( 1 minute)

5. Remove sample from stain, and allow excess stain to drip off. Sample will likely be darker than desired - if so place sample in cup containing 70\% ethanol until desired staining intensity is achieved.

6. Place sample on transparent grid with distal end on the right and proximal end on the left. 
7. Verify sample is mucosal side up. Striations in many directions will be visible and the resolution of crypts poor if mucosal side is down.

8. Keep the sample moist with cold PBS on a q-tip.

9. If mucin is present, remove with EXTREME CARE using a moist (PBS) q-tip.

II. Morphological characteristics of $\mathrm{ACF} / \mathrm{AC}$

Are characteristically larger ( 2-4 times) and are stained darker than normal crypts

$>$ Have enlarged/elongated lumens

$>$ May appear to be raised above the surrounding mucosa

$>$ May have an enlarged pericryptal zone

III. Scoring

1. Establish criteria for determining aberrant crypts (e.g. photo of AC/ACF for which a consensus has been reached).

2. Aberrant crypts are found as single crypts (AC) or multiples (ACF) and should be diagramed and tallied accordingly.

3. Begin at the distal end of the colon (should be to the observers right) and continue pattern (described in step 4 below) until the proximal end is reached.

4. Begin at the top of the distal colon (far edge, right end). Move from top to bottom. When done with this field, find a point of reference on the left edge of the field of view. Adjust the field of view proximally (left) until point of reference is on the right edge of the field of view. Proceed to score the crypt from bottom to top. When top is reached adjust field of view proximally as before.

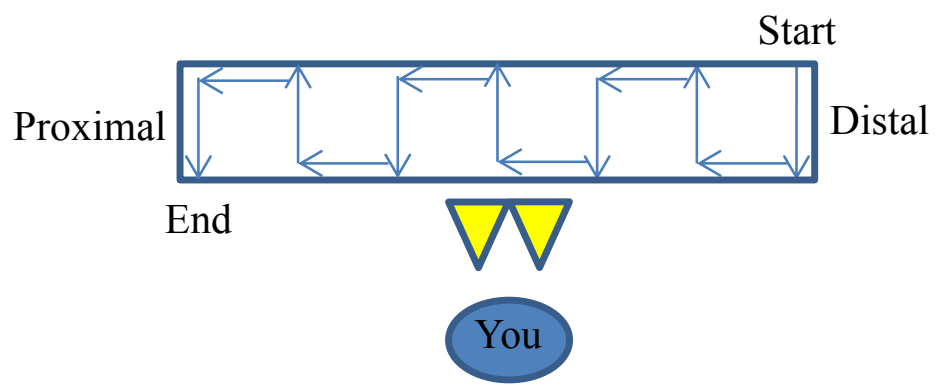

5. Once sample is completely scored, reveal and record the sample ID on the scoring sheet.

Protocol for Setup of ACF Microscope (Chapkin)

1. Turn on Computer and Monitor (Right side)

2. Turn on black box (Left side)

3. White box on top of black box (Left side)

4. Camera on top of microscope

5. Black box (Right side) 


\section{Chapter II measurement of apoptosis using TUNEL}

Kit: ApopTag Plus Peroxidase In Situ Apoptosis Detection Kit (S7101)

Manufacturer: EMD Millipore

Updated: 10/19/2017

Reagents required:

\begin{tabular}{|c|c|c|c|c|}
\hline Reagent/Kit & Manufacturer & Catalog \# & \#samples/kit, Qty & \$ per kit \\
\hline ApopTag Plus Kit & EMD Millipore & S7101 & 40 Samples (order 6) & 551.00 \\
\hline Proteinase $\mathrm{K}$ & EMD Millipore & 21627 & $15 \mathrm{ml}, 200 \mathrm{ug} / \mathrm{ml}$ & 102.00 \\
\hline Filters & EMD Millipore & SCGP00525 & 25 each & 160.00 \\
\hline $30 \% \mathrm{H}_{2} \mathrm{O}_{2}$ & Sigma-Aldrich & H1009-100ML & $100 \mathrm{~mL}$ & 113.00 \\
\hline Xylene & Sigma-Aldrich & 534056-4X4L & $4 \times 4$ Liters & 323.00 \\
\hline $100 \%$ n-butanol & Sigma-Aldrich & B7906-500ML & $500 \mathrm{~mL}$ & 49.40 \\
\hline DTT & Sigma-Aldrich & $43815-1 \mathrm{G}$ & $1 \mathrm{~g}$ & 92.30 \\
\hline DNase I & Sigma-Aldrich & D7291-.5MG & $.5 \mathrm{mg}$ & 181.00 \\
\hline Sodium acetate & Sigma-Aldrich & $71188-1 \mathrm{KG}$ & $1 \mathrm{~kg}$ & 98.10 \\
\hline Acetic acid & Sigma-Aldrich & 338826-100ML & $100 \mathrm{~mL}$ & 119.00 \\
\hline $\mathrm{MgCl}_{2}$ & Sigma-Aldrich & M2670-500G & $500 \mathrm{~g}$ & 69.10 \\
\hline Permount & $\begin{array}{l}\text { Electron } \\
\text { Microscopy } \\
\text { Sciences }\end{array}$ & $17986-05$ & $500 \mathrm{~mL}$ & 80.00 \\
\hline $100 \%$ Ethanol & Bio/Bio & Unknown & Unknown & Unknown \\
\hline $\begin{array}{l}\text { Methyl Green (no } \\
\text { crystal violet) }\end{array}$ & In House & & & \\
\hline $\mathrm{NaH}_{2} \mathrm{PO}_{4}$ & In House & & & \\
\hline $\mathrm{Na}_{2} \mathrm{HPO}_{4}$ & In House & & & \\
\hline $\mathrm{dH}_{2} \mathrm{O}$ & In House & N/A & N/A & N/A \\
\hline
\end{tabular}

\section{Reagent Preparation:}

1) 10X Phosphate Buffered Saline, pH 7.4 (PBS)

To make $1 \mathrm{~L}$, dissolve the following in $800 \mathrm{~mL} \mathrm{dH}_{2} \mathrm{O}$ :

$\begin{array}{ll}\mathrm{Na}_{2} \mathrm{HPO}_{4} & 55.0 \mathrm{~g} \\ \mathrm{NaH}_{2} \mathrm{PO}_{4} & 13.5 \mathrm{~g} \\ \mathrm{NaCl} & 117.0 \mathrm{~g}\end{array}$

Adjust the $\mathrm{pH}$ to 7.4 using $\mathrm{NaOH}$ or $\mathrm{HCl}$ and add $\mathrm{dH}_{2} \mathrm{O}$ to a final volume of $1000 \mathrm{~mL}$.

2) $0.1 \%(w: v)$ Methyl Green

a. Prepare $0.1 \mathrm{M}$ sodium acetate, $\mathrm{pH} 4.0$

1. Dissolve $1.36 \mathrm{~g} \mathrm{C}_{2} \mathrm{H}_{3} \mathrm{O}_{2} \mathrm{Na} \bullet 3 \mathrm{H}_{2} \mathrm{O}$ in $80 \mathrm{~mL}$ of $\mathrm{dH}_{2} \mathrm{O}$.

2. Adjust $\mathrm{pH}$ to 4.0 with acetic acid. Add $\mathrm{dH}_{2} \mathrm{O}$ to a final volume of $100 \mathrm{~mL}$

3. Dissolve $0.1 \mathrm{~g}$ of methyl green in $100 \mathrm{~mL}$ of $0.1 \mathrm{M}$ sodium acetate, $\mathrm{pH} 4.0$ (last step).

4. Filter through a 0.45 or 0.2 -micron filter prior to use.

3) Proteinase $\mathrm{K}$

a. Dilute the $200 \mathrm{ug} / \mathrm{mL}$ stock of Proteinase K (21627) to $20 \mathrm{ug} / \mathrm{mL}$ in PBS just before use.

1.Direct slide application- $100 \mathrm{ul}$ test of diluted stock is required per section.

4) Working Strength Hydrogen Peroxide $\left(\mathrm{H}_{2} \mathrm{O}_{2}\right)$

a. Dilute the $30 \% \mathrm{H}_{2} \mathrm{O}_{2}$ stock to $3 \% \mathrm{H}_{2} \mathrm{O}_{2}$ in $\mathrm{PBS}$ (i.e. 10-fold dilution). 
1. Direct slide application- $100 \mathrm{ul}$ is required per $5 \mathrm{~cm}^{2}$ specimen (section).

\section{5) DN Buffer (30 mM Tris Base, pH 7.2, 4 mM MgCl, 0.1 mM DTT)}

a. Prepare $1 \mathrm{M}$ Tris Base, $\mathrm{pH} 7.2$

1. Dissolve $12.1 \mathrm{~g}$ of Tris base in $80 \mathrm{~mL}$ of $\mathrm{dH}_{2} \mathrm{O}$. Adjust the $\mathrm{pH}$ to 7.2 with concentrated $\mathrm{HCl}$ and add $\mathrm{dH}_{2} \mathrm{O}$ to a final volume of $100 \mathrm{~mL}$.

b. Prepare $1 \mathrm{M} \mathrm{MgCl}_{2}$

1. Dissolve $20.3 \mathrm{~g}$ of $\mathrm{MgCl}_{2} \bullet 6 \mathrm{H}_{2} \mathrm{O}$ in sufficient $\mathrm{dH}_{2} \mathrm{O}$ for a final volume of $100 \mathrm{~mL}$.

c. Prepare DN buffer

1. Combine $3 \mathrm{~mL}$ of $1.0 \mathrm{M} \underline{\text { Tris }}, \mathrm{pH} 7.2,400 \mathrm{uL}$ of $1.0 \mathrm{M} \mathrm{MgCl}_{2}, 1.54 \mathrm{mg}$ of DTT and sufficient $\underline{\mathrm{d}}_{2} \mathrm{O}$ for a final volume of $100 \mathrm{~mL}$.

Kit Supplied Reagent Preparation

\begin{tabular}{|l|c|c|}
\hline \multicolumn{1}{|c|}{ Reagent } & Vol/cm & Vol/5 $\mathbf{~ c m}^{\mathbf{2}}$ \\
\hline Equilibration Buffer & $13 \mathrm{ul}$ & $65 \mathrm{ul}$ \\
\hline Working Strength TdT & $11 \mathrm{ul}$ & $55 \mathrm{ul}$ \\
\hline Anti-digoxigenin-Peroxidase & $13 \mathrm{ul}$ & $65 \mathrm{ul}$ \\
\hline DAB Peroxidase Substrate & $15 \mathrm{ul}$ & $75 \mathrm{ul}$ \\
\hline
\end{tabular}

1) Working Strength TdT Enzyme

The concentrated TdT Enzyme provided in the kit is supplied in a stabilization buffer to preserve activity. It must be diluted with Reaction Buffer prior to use. Use 1:80 dilution of TdT enzyme in Reaction Buffer. To prepare, add in a fresh microcentrifuge tube:

1 . \# Sections x $45 \mathrm{uL}=$ Total uL

2. Total uL *.9875 = uL REACTION BUFFER

3. Total $\mathrm{uL} * .0125=$ uL TdT ENZYME

Mix well by vortexing. This reagent may be prepared in advance and stored on ice for no more than 6 hours. This amount is sufficient to treat two $5 \mathrm{~cm}^{2}$ tissue specimens.

2) Working Strength Stop/Wash Buffer

Prepare by adding:

$1 \mathrm{~mL}$ Stop/Wash Buffer (90419)

$34 \mathrm{~mL} \quad \mathrm{dH}_{2} \mathrm{O}$

$35 \mathrm{~mL}$ Total

This amount is sufficient to treat 5 slides in a coplin jar. This reagent may be prepared in advance and stored in a glass or plastic container at $4^{\circ} \mathrm{C}$ for up to 1 year. Use a fresh aliquot for each experiment.

3) Working Strength Peroxidase Substrate

Prepare working strength DAB by mixing in a clean tube:

147 uL DAB Dilution Buffer (90424)

3 uL DAB Substrate (90423)

$150 \mathrm{uL}$ Total

After dilution, warm the mixture to room temperature and store in the dark until use. This amount is sufficient to treat two $5 \mathrm{~cm}^{2}$ tissue specimens. After mixing, the solution is stable for 7 days at room temperature or up to 14 days at $4^{\circ} \mathrm{C}$.

4) Protocol for Positive Control Preparation using DNase I 
This step is to be performed on a deparaffinized, rehydrated tissue (Primary protocol step 1), which has been treated with Proteinase K (Primary protocol step 2), but prior to Peroxidase quenching (Primary protocol step 3).

1. Pretreat section with DN Buffer (30 mM Trizma base, $\mathrm{pH} 7.2,4 \mathrm{mM} \mathrm{MgCl}, 0.1 \mathrm{mM}$ DTT) at room temperature for 5 minutes.

2. Dissolve DNase I in DN Buffer to a final concentration of $1.0-0.1 \mathrm{ug} / \mathrm{mL}$ (specific activity is $10,000 \mathrm{U} / \mathrm{mL}-1,000 \mathrm{U} / \mathrm{mL})$.

3. Apply DNase solution and incubate for 10 minutes at room temperature.

4. Rinse with 5 changes of $\mathrm{dH}_{2} \mathrm{O}$ for 3 minutes each change.

\section{ApopTag Protocol \\ ***DO NOT ALLOW SAMPLES TO DRY OUT DURING PROCESSING***}

\section{1) Deparaffinize Tissue Section (in a coplin jar)}
a. XYLENE; 5 min ea. (1-3)
b. Let XYLENE just dry and circle sections with PAP Pen, dry $1 \mathrm{~min}$.
c. __ _ ABSOLUTE ETOH; 5 min ea. (4-5)
d. $95 \% \mathrm{ETOH} ; 3 \min (6)$
e. $\quad 70 \% \mathrm{ETOH} ; 3 \mathrm{~min}(7)$
f. 1X PBS; $5 \min (8)$

\section{2) Pretreat Tissue}

a. Apply freshly diluted PROTEINASE K (20 ug/mL) to the specimen for $15 \mathrm{~min}$. at RT directly on the slide ( $50 \mathrm{ul} / \mathrm{section})$.
i. $100 \mathrm{ul} / \mathrm{section} \rightarrow$ (\#sections+1) $\times 100 \mathrm{ul}=$ Total Prot. K needed

ii. Total Prot. K $\mathrm{x} .1=$ Prot $\mathrm{K}$ stock needed

iii. Total Prot. $\mathrm{K}$ $\mathrm{x} .9=$ PBS needed

b. $\mathrm{dH}_{2} \mathrm{O} ; 2$ min ea. (9-10)

3) Perform DNase I treatment if sample is to be used as positive control (Page 3)

4) Quench Endogenous Peroxidase

a. Quench in 3\% hydrogen peroxide in PBS (Page 2) for $5 \mathrm{~min}$.

i. $100 \mathrm{ul} / \mathrm{section} \rightarrow$ __ (\# sections+1) $\times 100 \mathrm{ul}=$ Total ul 3\% needed

ii. Total ul 3\% $* .1=$ ul $30 \% \mathrm{H}_{2} \mathrm{O}_{2}$

iii. Total ul 3\% $* .9=$ ul $1 \mathrm{X}$ PBS

b. 1X PBS; 5 min ea. (11-12)

\section{5) Apply Equilibration Buffer}

a. ___ Gently tap off excess liquid and carefully blot or aspirate around section. (Do this step and next together, 1 slide at a time, to avoid drying sample)

b. Apply $60 \mathrm{ul} / \mathrm{section}$ of EQUILIBRATION BUFFER directly onto specimen.

i. \# sections $\times 60 \mathrm{ul} / \mathrm{section}=$ ul total

c. Incubate for at least 10 seconds at RT. Seconds incubated

\section{6) Apply Working Strength TdT Enzyme}

a. Gently tap off excess liquid and carefully blot or aspirate around section.

b. Immediately pipette: i. __ $60 \mathrm{ul} / \mathrm{section}$ of WORKING STRENGTH TdT ENZYME (1:80) or 


$$
\begin{aligned}
& \text { 1. } \quad \text { \# Sections } x 45 \mathrm{uL}=\_ \text {Total } \mathrm{uL} \\
& \text { 2. Total } \mathrm{uL} * .9875=\_ \text {uL REACTION BUFFER } \\
& \text { 3. Total } \mathrm{uL} * .0125=\_ \text {uL TdT ENZYME } \\
& \text { ii. } \quad \text { T0 ul REACTION BUFFER (negative controls). }
\end{aligned}
$$

c. Incubate in humidified chamber at $37^{\circ} \mathrm{C}$ for 1 hour.

\section{7) Apply Stop/Wash Buffer}

a. ___ Agitate slides for 15 seconds in WORKING STRENGTH STOP/WASH BUFFER in a coplin jar then incubate for $10 \mathrm{~min}$ at RT. (13)

b. Remove an aliquot of ANTI-DIGOXIGENIN CONJUGATE from the stock vial sufficient to process the desired number of specimens. Warm the aliquot to RT.

i. \# sections $\times 60 \mathrm{ul} / \mathrm{section}=$ Total ul Aliquot

\section{8) Apply Anti-Digoxigenin Conjugate}

a. __ _ 1X PBS; 1 min ea. (14-16)

b. Gently tap off excess liquid and carefully blot or aspirate around the section.

c. Apply RT Anti-Digoxigenin Conjugate (60 ul/section).

d. Incubate in a humidified chamber for $30 \mathrm{~min}$. at RT.

\section{9) Wash in PBS}

a. 1X PBS; 2 min ea. RT (17-20)

b. While slides are washing, prepare WORKING STRENGTH PEROXIDASE SUBSTRATE.
i. \# Sections x $60 \mathrm{uL}=$ Total uL WSPS
ii. Total uL WSPS *.0125 = uL DAB Substrate
iii. Total uL WSPS
Buffer

\section{0) Develop Color in Peroxidase Substrate}

a. Gently tap off excess liquid and carefully blot or aspirate around the section.

b. Apply $60 \mathrm{uL} /$ section of PEROXIDASE SUBSTRATE to cover specimen.

c. __ Stain for $45 \mathrm{~s}$ at RT. Humid chamber not necessary. min stained.

d. In order to obtain optimal staining time, observe under microscope.

\section{1) Wash Specimen}
a.
b. $\mathrm{dH}_{2} \mathrm{O} ; 1$ min ea. (21-23) $\mathrm{dH}_{2} \mathrm{O} ; 5$ min RT (24)

\section{2) Counterstain Specimen}
a. 0.1\% Methyl green; $15 \mathrm{sec}$, RT (25)
b. --- $\mathrm{dH}_{2} \mathrm{O}$; Dip 10 times ea. (1 and 2), 30 secs no agitation (3) (26-28) 31) N-BUTANOL; Dip 10 times ea. (1 and 2), 30 secs no agitation (3) (29-

\section{3) Mount Specimen}

a. XYLENE; 2 min ea. (32-34)

b. Remove slides 1 at a time from jar. Gently tap the side to drain, but do not allow to dry!

c. Apply Permount and then apply cover glass.

d. Dry 24 hours before viewing under microscope. 


\section{Chapter II measurement of gene expression using real-time PCR}

Scraped mucosal samples were obtained at termination, placed into RNA protective solution and stored at $-80^{\circ} \mathrm{C}$ until further analysis. Total RNA was isolated from mucosal samples using the RNAqueous ${ }^{\mathrm{TM}}$ Total RNA Isolation Kit (Invitrogen, AM1912) followed by DNase treatment (DNA-free ${ }^{\mathrm{TM}}$ Kit, Ambion, AM1906). Eppietubes containing homogenized scraped mucosa and Denaturation Solution were removed from $-80^{\circ} \mathrm{C}$ storage and thawed on ice.

Total RNA was isolated using the following protocol:

A. Before Using the Kit for the First Time

a. Prepare 64\% Ethanol Solution-Add $38.4 \mathrm{~mL}$ of $100 \%$ ethanol (ACS grade or equivalent) to the bottle labeled "Water for 64\% Ethanol," which contains 21.6 $\mathrm{mL}$ of RNase-free water. Mix well, and mark the empty box on the label to indicate that the ethanol was added.

b. Prepare Wash Solution \#2/3-Add $64 \mathrm{~mL}$ of $100 \%$ ethanol (ACS grade or equivalent) to the bottle labeled "Wash Solution \#2/3 Concentrate." Mix well, and mark the empty box on the label to indicate that the ethanol was added.

B. Work Area and Equipment Preparation

a. Lab Bench and Pipettors-Before working with RNA, clean the lab bench, and pipettors with an RNase decontamination solution such as Ambion RNase Zap.

b. Gloves and RNase-free technique-Wear laboratory gloves at all times during this procedure and change them frequently. They will protect you from the reagents, and they will protect the RNA from nucleases that are present on the skin. Use RNase-free pipette tips to handle the kit reagents.

c. Washing/sterilization of equipment-The equipment used for tissue disruption/homogenization should be washed well with detergent and rinsed thoroughly. Baking is unnecessary, because the Lysis/Binding Solution will inactivate any low level RNase contamination.

I. As you start procedure:

1. Heat an aliquot of Elution Solution (typically 50-200 uL per prep) in an RNase-free microcentrifuge tube in a heat block set to $70-80^{\circ} \mathrm{C}$.

2. Briefly inspect the filter cartridges before use. Occasionally, the glass fiber filters may become dislodged during shipping. If this is the case, gently push the filter down to the bottom of the cartridge using the wide end of an RNase-free pipette tip.

II. Preparation of the lysate for RNA isolation

1. Ensure lysate is somewhat viscous, but if the solution is very viscous, or contains gelatinous material (likely genomic DNA), then it should be sonicated, homogenized in a rotor-stator homogenizer, or passed through a 25 gauge syringe needle several times until the viscosity is reduced. 
III. Filter Binding, Washing, and Elution of RNA

1. Add an equal volume (for PLUM $\rightarrow 500 \mathrm{ul}$ ) of $64 \%$ Ethanol and mix by carefully pipetting or vortexing.

2. Apply up to $700 \mathrm{ul}$ of the lysate/ethanol mixture to a Filter Cartridge assembled in a collection tube.

3. Centrifuge at RCF $10,000-15,000 \times$ g (typically $10,000-14,000 \mathrm{rpm}$ ) for $\sim 45$ seconds.

4. Discard the flow-through and, if necessary, add any remaining lysate/ethanol mixture to the column. Centrifuge as before and discard flow-through.

5. Apply $700 \mathrm{ul}$ Wash Solution \#1 to the filter cartridge.

6. Centrifuge at RCF $10,000-15,000 \times \mathrm{g}$ (typically $10,000-14,000 \mathrm{rpm}$ ) for $\sim 45$ seconds. Discard flow-through.

7. Add $500 \mathrm{ul} \mathrm{Wash} \mathrm{Solution} \mathrm{\# 2/3.}$

8. Centrifuge at RCF $10,000-15,000 \times \mathrm{g}$ (typically $10,000-14,000 \mathrm{rpm}$ ) for $\sim 45$ seconds. Discard flow-through.

9. Repeat Step 7 and 8 with a second 500 ul aliquot of Wash Solution \#2/3.

10. Centrifuge at RCF $10,000-15,000 \times \mathrm{g}$ (typically $10,000-14,000 \mathrm{rpm}$ ) for $\sim 45$ seconds. This additional drying step ensures no wash solution remains in the filter.

11. Place Filter Cartridge into a fresh Collection Tube. Discard old Collection Tube.

12. Pipet preheated Elution Solution to the center of the filter. This step is carried out with two sequential elutions. First pipet $40 \mathrm{ul}$ of preheated Elution Solution ( $1^{\text {st }}$ step of sequential elution) onto the center of the column. Recover eluate by centrifugation for $\sim 30$ seconds at room temperature (RCF 10000-15000 x g).

13. Pipet $20 \mathrm{ul}$ of preheated Elution Solution ( $2^{\text {nd }}$ step of sequential elution). Recover eluate by centrifugation as in step 11 .

14. Proceed to DNase Treatment.

DNase treatment protocol

1. Add .1 Volume (for $60 \mathrm{ul}$ elution $\rightarrow \underline{6 \mathrm{ul}}$ ) $10 \mathrm{X}$ DNase I Buffer and $\underline{1 \mathrm{ul}}$ rDNase I to the RNA, and mix gently.

2. Incubate at $37^{\circ} \mathrm{C}$ for $20-30 \mathrm{~min}$.

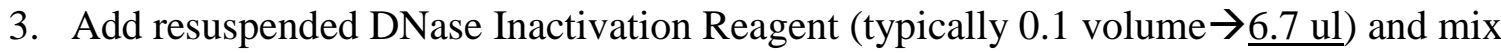
well.

4. Incubate $2 \mathrm{~min}$ at room temperature, mixing occasionally.

5. Centrifuge at $10,000 \mathrm{x} \mathrm{g}$ for $1.5 \mathrm{~min}$ and transfer the RNA to a fresh RNase free tube.

6. Aliquot 4 ul into RNase free PCR tube for Nanodrop and Agilent QC.

Following DNase treatment, the concentration of the isolated RNA was assessed on the

Nanodrop instrument and the quality (RIN, RNA integrity number) was assessed on the Agilent

Bioanalyzer (448). After quantitation and quality assessment of total RNA, 1100 ng of cDNA

was prepared for each total RNA sample using the SuperScript III First-Strand Synthesis System 
for RT-PCR kit (Life Technologies, 18080-051). The following protocol was used, which is a

slightly modified version of the manufacturers' protocol.

1) Add the following components to a nuclease-free microcentrifuge tube: $1 \mu 1$ of oligo(dT)20 $(50 \mu \mathrm{M}), 50-250 \mathrm{ng}$ of random primers, $10 \mathrm{pg}-5 \mu \mathrm{g}$ total RNA, $1 \mu 110 \mathrm{mM}$ dNTP Mix (10 mM each dATP, dGTP, dCTP and dTTP at neutral $\mathrm{pH})$, sterile, distilled water to $13 \mu$ l.

2) Heat mixture to $65^{\circ} \mathrm{C}$ for 5 minutes and incubate on ice for at least 1 minute.

3) Collect the contents of the tube by brief centrifugation and add: $4 \mu 15 \mathrm{X}$ First-Strand Buffer, $1 \mu 10.1$ M DTT, $1 \mu 1$ RNaseOUT ${ }^{\text {TM }}$ Recombinant RNase Inhibitor (Cat. no. 10777-019, 40 units/ $\mu 1), 1 \mu 1$ of SuperScript ${ }^{\mathrm{TM}}$ III RT (200 units/ $\left.\mu \mathrm{l}\right)$.

4) Mix by pipetting gently up and down. If using random primers, incubate tube at $25^{\circ} \mathrm{C}$ for 5 minutes.

5) Incubate at $50^{\circ} \mathrm{C}$ for 60 minutes.

6) Inactivate the reaction by heating at $70^{\circ} \mathrm{C}$ for 15 minutes.

7) cDNA was used directly in a PCR reaction or stored at $-20^{\circ} \mathrm{C}$.

Real-time PCR was performed on select genes (Tlr2, Tlr4, Tollip, Slc5a8, Slc16a1, Nfkb1,

Nfkbia, Ptgs2 (Cox2), Acads, Acadm, Muc1, Muc2, Muc3a, Muc4, Muc5ac, 18s rRNA, and

Gapdh) using Taqman ${ }^{\circledR}$ Array Micro Fluidic Cards (Applied Biosystems, Foster City, CA) and a

ABI 7900 HT thermocycler (Applied Biosystems, Foster City, CA) using the following protocol:

1) For each sample, determine the total number of reservoirs to be filled, based on the format of your TaqMan Array card. Prepare the PCR reaction mix (cDNA master mix). Fill the TaqMan Array card with the reaction mix. Centrifuge the TaqMan Array card. Seal the TaqMan Array card.

2) Calculate the total volume required for each reaction component.

3) If frozen, thaw the cDNA samples on ice. Resuspend the cDNA samples by inverting the tube, then gently vortexing.

4) Mix the master mix thoroughly by swirling the bottle.

5) For each sample, label a $1.5-\mathrm{mL}$ microcentrifuge tube, then add the required components to the labeled tube.

6) Cap the microcentrifuge tubes, then gently vortex the tubes to thoroughly mix the solution.

7) Briefly centrifuge the tubes to spin down the contents and eliminate air bubbles.

8) Fill each fill reservoir with sample-specific PCR mix made from a single cDNA sample $(1000 \mathrm{ng})+$ nuclease-free water, 50.0 microliters TaqMan ${ }^{\circledR}$ Gene Expression Master Mix.

9) Allow the TaqMan Array card to reach room temperature, then carefully remove it from its packaging. A minimum of 15 minutes at room temperature is required to equilibrate the card. 
10) Place the TaqMan Array card on a lab bench, with the foil side down.

11) Load $100 \mu \mathrm{L}$ of the desired sample-specific PCR reaction mix into a $100-\mu \mathrm{L}$ micropipette.

12) Hold the micropipette at an angled position and place the tip in the fill port (the larger of the two holes) IMPORTANT! Do not allow the tip to contact and possibly damage the coated foil beneath the fill port.

13) Dispense the sample-specific PCR reaction mix so that it sweeps in and around the fill reservoir toward the vent port. IMPORTANT! Pipette the entire $100 \mu \mathrm{L}$ into the fill reservoir. Be careful when pushing the micropipette plunger to its second stop position (to expel the sample-specific PCR reaction mix from the tip). If a large amount of air is released, it can push the reaction mix out of the fill reservoir via the vent port or introduce bubbles into the fill reservoir.

14) After the fill reservoirs have been filled with the sample-specific PCR reaction mix, centrifuge the TaqMan Array card to distribute the reaction mix to the reaction wells. IMPORTANT! You must use a Sorvall@ or Heraeus centrifuge with the Sorvall/Heraeus Custom Buckets and card holders. The Custom Buckets and card holders are custommade for the TaqMan Array cards. Do not use any other centrifuge or bucket/card holder system for this procedure.

15) Place TaqMan Array cards into the Sorvall/Heraeus buckets:

a. Obtain an empty Sorvall/Heraeus Custom Bucket and card holder.

b. Place the bucket on a lab bench, with the label facing you.

c. Insert TaqMan Array cards into the card holder, making sure that the fill reservoirs project upwards out of the card holder and the reaction wells face the same direction as the "This Side Out" label. You use blank balance cards to fill any open positions in the card holder. Use the blank balance cards provided with the installation kits. IMPORTANT! Be sure to use the blank balance cards to fill any open positions. The blank balance cards will balance the centrifuge and prevent damage to the card holder. If the card holder is not completely filled, the TaqMan Array card may become displaced during centrifugation, resulting in uneven filling.

d. Place a filled card holder in the bucket so that the "This Side Out" label faces out.

16) Set the centrifuge:

a. Power on the centrifuge.

b. Use the front panel controls to set the bucket type to 15679. IMPORTANT! To ensure that the maximum rotational speed stays within the manufacturer's specified limits, be sure to set the correct bucket type.

c. Use the front panel controls to set the following operations parameters: Parameter EASYSet (touchpad), Up ramp rate 9, Down ramp rate 9, Rotational speed 1200 rpm $(331 \times \mathrm{g}) 1200 \mathrm{rpm}$, Centrifugation time $2 \times 1 \mathrm{~min}$.

17) Place the buckets into the centrifuge:

a. Press the Open button on the centrifuge to open the centrifuge cover.

b. Place each loaded bucket onto an open rotor arm of the centrifuge. Make sure each bucket can swing easily within its slotted position on the rotor arm. IMPORTANT! The manufacturer recommends running the centrifuge with all four buckets. If the buckets are not fully loaded with TaqMan Array cards 
containing the sample-specific PCR reaction mix, place blank balance cards and card holders into the buckets. Make sure the buckets and their contents are balanced; opposing buckets should have matching weights.

c. Close the centrifuge cover.

18) Run the centrifuge:

a. Press the Start button. The centrifuge starts, then automatically stops after $1 \mathrm{~min}$, per the programmed sequence.

b. Repeat step a so that the TaqMan Array cards are centrifuged for a total of two consecutive, 1minute spins. IMPORTANT! To ensure complete distribution of the PCR reaction mix, you must centrifuge the TaqMan Array cards for a total of two consecutive, 1-minute spins.

19) Remove the TaqMan Array cards:

a. Press the Open button.

b. When the cover has fully opened, remove the buckets from the centrifuge, then remove the card holders from the buckets.

c. Remove all TaqMan Array cards from the buckets by gently lifting them by their carrier sides.

20) Examine the TaqMan Array cards to be sure filling is complete. The amount of PCR reaction mix remaining in the fill reservoirs should be consistent from reservoir to reservoir.

21) Seal the TaqMan Array Card. Position the sealer:

a. Place the sealer on a sturdy lab bench, approximately waist high so that it can be easily used.

b. Turn the sealer so that the front end (the "starting position" shown below) is closest to you and the back end is farthest from you. In the correct position, the arrows on the sealer are pointing away from you.

c. Place the sealer's carriage in its starting position. IMPORTANT! Never insert a TaqMan Array card into the sealer if the carriage is not in its starting position. The TaqMan Array card will be irreparably damaged if the carriage is moved across it toward its starting position.

22) Insert a TaqMan Array card into the sealer:

a. Orient the TaqMan Array card in the proper direction over the sealer's insert plate. The card's fill reservoir end should be the end closest to the arrows etched in the base of the sealer.

b. Line up the card's rear pin grooves, foil side up, to the stylus pins on the sealer.

c. Gently place the card on top of the insert plate and ensure that the front end of the card is held securely in place by the spring clips.

d. Gently push the card until it is seated securely in the insert plate. Note: When properly seated, the TaqMan Array card's foil surface should be level with the base of the sealer. The four spring clips ensure that the card is held in the proper position.

e. Push the carriage across the base of the sealer in the direction of the arrows. Use a slow, steady, and deliberate motion to push the carriage across the entire length of the TaqMan Array card until the carriage reaches the mechanical stops. It is important to avoid moving the carriage rapidly across the card. The sealer has 
mechanical stops at both ends to prevent the carriage from coming off. Therefore, do not use excessive force or speed when pushing the carriage. IMPORTANT! Do not move the carriage back before removing the TaqMan Array card.

23) Remove the sealed TaqMan Array card by grasping its sides and lifting it off the sealer's insert plate. In the middle of the sealer's insert plate, there is a thumb slot to help you easily access one side of the card.

24) Inspect the TaqMan Array card for proper sealing. The indentations from the stylus assembly should match up with the card's main channels. If the indentations do not match up, or if the foil is in any way damaged, do not use the TaqMan Array card.

25) Using scissors, trim the fill strip from the TaqMan Array card. Use the edge of the card's carrier as a guide. After the TaqMan Array cards have been loaded and sealed, they are stable for at least 64 hours.

26) Set Up the SDS Plate Document. Start SDS Software v2.1 or later.

27) Select File, New.

28) Complete the New Document dialog box:

a. From the Assay dropdown menu, select $\Delta \Delta \mathrm{CT}$ (RQ) or Relative Quantification.

b. From the Container dropdown menu, select 384 Wells TaqMan Low Density Array.

c. Complete the remaining fields as shown, then click OK.

29) Import the SDS Setup File (*.txt) into the new SDS plate document:

a. In the $\mathrm{CD}$ drive, insert the Array Information $\mathrm{CD}$ that shipped with your TaqMan Array card.

b. In the SDS software, select FileImport. In the SDS software, set up the experiment (SDS plate document), using the SDS Setup File included on the Array Information CD (this page).

c. In the Import dialog box, navigate to the SDS Setup File for your TaqMan Array card, then click Import. The SDS software imports information from the SDS Setup File into the SDS plate document. Note: The SDS software uses the information from the SDS Setup File to automatically configure the plate grid and setup table with detector, detector task, marker, and sample data. For details on the SDS Setup File, see page 26. IMPORTANT! Modifying the contents of the SDS Setup File can corrupt the file, making the file unusable (that is, you will not be able to access information for the TaqMan Array card).

30) Save the SDS plate document:

a. Select File, Save As.

b. Navigate to a save location.

c. Enter a name for the SDS plate document.

d. For Files of Type, select SDS 7900HT Document (*.sds) or SDS 7900HT Template Document (*.sdt). Note: You can save the plate document as an SDS plate document $(*$.sds) or SDS template $(*$.sdt). Saving the plate document as an SDS template is recommended when you want to create duplicate plate documents for a series of TaqMan Array cards with identical assay configurations.

e. Click Save.

31) Open the SDS plate document in the SDS software. 
32) Select the Instrument tab.

33) Use the default thermal cycling conditions, as shown in the Thermal Cycler tab. Note: When you selected 384 Well TaqMan Low Density Array, the SDS software automatically set the appropriate thermal cycling conditions for the TaqMan Array cards.

34) Select the Real-Time tab. If the software is not connected to the instrument, click Connect to Instrument.

35) If the instrument tray is inside the instrument, click Open/Close to rotate the instrument tray to the OUT position.

36) Verify that the TaqMan Array Micro Fluidic Card Thermal Cycling Block is installed in the instrument tray.

37) Place the prepared TaqMan Array card in the instrument tray with: Well A1at the top left corner of the tray and the notched corner at the top right and the barcode toward the front of the instrument.

38) Click Start Run. The instrument tray rotates to the IN position. During the run, the instrument displays real-time status information in the Instrument Real-Time tabs and records the fluorescence emissions. During the run, you can view the data (as the data are generated in real-time).

39) When the Run Complete dialog box appears, click OK to close the dialog box, click Open/Close, then remove the TaqMan Array card from the instrument tray.

Expression levels were normalized to $18 \mathrm{~S}$ gene expression.

Table B-1. Assay ID for selected gene targets in Chapter II.

\begin{tabular}{lll}
\hline Gene Symbol & Alias & Assay ID \\
\hline Tlr2 & - & Rn02133647_s1 \\
\hline TIr4 & - & Rn00569848_m1 \\
\hline Tollip & - & Rn01479669_m1 \\
\hline 18s rRNA & - & Hs99999901_s1 \\
\hline Slc5a8 & RGD1564146 & Rn01503812_m1 \\
\hline Slc16a1 & MCT1;RATMCT1;RNMCT11 & Rn00562332_m1 \\
\hline Nfkb1 & NF-kB & Rn01399583_m1 \\
\hline Nfkbia & RL/IF-1 & Rn01473657_g1 \\
\hline Ptgs2 & COX-2;Cox2 & Rn01483828_m1 \\
\hline Acads & Scad & Rn00574634_m1 \\
\hline Acadm & MCAD & Rn00566390_m1 \\
Muc1 & - & Rn01462585_m1 \\
Muc2 & HH-Muc & Rn01498196_g1 \\
\hline Muc3a & Muc3 & Rn01481134_m1 \\
\hline Muc4 & ASGP-1;Psmc & Rn01475265_m1 \\
\hline Muc5ac & - & Rn01451270_g1 \\
\hline
\end{tabular}




\section{Chapter II microbial DNA isolation and quantification for sequencing}

Luminal contents collected at termination (Chapter II) were immediately transferred to sterile cryotubes, placed on ice, then stored at $-80^{\circ} \mathrm{C}$. DNA was isolated from homogenized fecal samples using a FastDNA SPIN kit for Feces according to the manufacturer's instructions (MP Biomedicals, Solon, OH). Fecal samples were weighed and then homogenized in the FastPrep 24 instrument with kit provided buffers (825 ul Sodium Phosphate Buffer, 275 ul PLS solution, 122 ul MT Buffer) in Lysing Matrix E tubes. Following homogenization, tubes were centrifuged at $14,000 \mathrm{x} \mathrm{g}$ for $5 \mathrm{~min}$, and the supernatant was transferred to a clean $2.0 \mathrm{ml}$ centrifuge tube. Proteins were precipitated from the supernatant by adding $250 \mathrm{ul}$ PPS solution and incubating at $4^{\circ} \mathrm{C}$ for $10 \mathrm{~min}$. Solution was centrifuged at $14,000 \mathrm{x}$ g for $2 \mathrm{~min}$, and the supernatant was transferred to $15 \mathrm{ml}$ conical tubes containing $1 \mathrm{ml}$ Binding Matrix Solution and placed on a plate rocker for $3 \mathrm{~min}$. Following a 2 min centrifugation at 14,000 x g, the supernatant was decanted

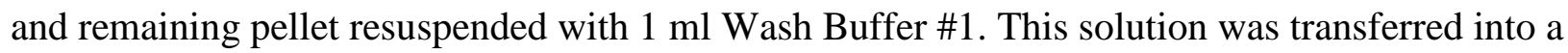
kit provided SPIN filter tube and centrifuged for $1 \mathrm{~min}$ at $14,000 \mathrm{x}$. A $500 \mathrm{ul}$ aliquot of Wash Buffer \#2 was added to the SPIN filter and all flow through was discarded following a 2 min centrifugation at $14,000 \mathrm{x}$ g. The SPIN filter was then transferred to a clean catch tube. To elute purified DNA, 50 ul of TES was added to the SPIN filter and the tubes were centrifuged at $14,000 \mathrm{x} g$ for $2 \mathrm{~min}$. Purified DNA was stored at $-80^{\circ} \mathrm{C}$. A negative control containing $\mathrm{H} 2 \mathrm{O}$ instead of sample was purified in parallel to each extraction batch to screen for contamination of extraction reagents.

Double-stranded DNA was quantified using the Quant-iT ${ }^{\mathrm{TM}}$ PicoGreen $^{\mathrm{TM}}$ dsDNA Assay Kit (Invitrogen, P11496) according to the manufacturers' protocol. To begin, a 'high-range' (1 $\mathrm{ng} / \mathrm{ml}$ to $1 \mu \mathrm{g} / \mathrm{ml}$ ) standard curve was set up. First, a $2 \mu \mathrm{g} / \mathrm{ml}$ stock solution of dsDNA in TE 
buffer was made. The lambda DNA standard, provided at $100 \mu \mathrm{g} / \mathrm{mL}$ in the Quant-iT ${ }^{\mathrm{TM}}$

PicoGreen ${ }^{\circledR}$ Kits, was diluted 50 -fold in TE to make the $2 \mu \mathrm{g} / \mathrm{mL}$ working solution. Thirty $\mu \mathrm{L}$ of the DNA standard mixed with $1.47 \mathrm{~mL}$ of TE was sufficient for the standard curve described below.

\begin{tabular}{|c|c|c|c|}
\hline Volume $(\mu \mathrm{l})$ of TE & $\begin{array}{c}\text { Volume }(\mu \mathrm{l}) \\
\text { of } 2 \mu \mathrm{g} / \mathrm{ml} \text { DNA Stock }\end{array}$ & $\begin{array}{c}\text { Volume }(\mu \mathrm{l}) \text { of } \\
\text { Diluted Quant-iT' } \\
\text { PicoGreen Reagent }\end{array}$ & $\begin{array}{c}\text { Final DNA } \\
\text { Concentration in } \\
\text { Assay }\end{array}$ \\
\hline 0 & 1000 & 1000 & $1 \mu \mathrm{g} / \mathrm{ml}$ \\
\hline 900 & 100 & 1000 & $100 \mu \mathrm{g} / \mathrm{ml}$ \\
\hline 990 & 10 & 1000 & $10 \mathrm{ng} / \mathrm{ml}$ \\
\hline 999 & 1 & 1000 & $1 \mathrm{ng} / \mathrm{ml}$ \\
\hline 1000 & 0 & 1000 & blank \\
\hline
\end{tabular}

1) Prepare the Quant-i ${ }^{\mathrm{TM}}$ PicoGreen $^{\circledR}$ Reagent by making a 200 -fold dilution of the concentrated DMSO solution in TE. To assay 20 samples in a $2 \mathrm{ml}$ final volume, add 100 $\mu \mathrm{l}$ Quant-i $\mathrm{T}^{\mathrm{TM}}$ PicoGreen ${ }^{\circledR}$ Reagent to $19.9 \mathrm{mk}$ TE. Cover the vial containing the prepared reagent in foil.

2) Dilute the stock solution into disposable cuvettes (or plastic test tubes) according to the table above.

3) Add $1.0 \mathrm{ml}$ of the aqueous working solution of PicoGreen ${ }^{\circledR}$ reagent. Mix well and incubate for 2 to 5 minutes at room temperature, protected from light.

4) After incubation, measure the sample fluorescence using a fluorescence microplate reader and stand fluorescein wavelengths (excitation $\sim 480 \mathrm{~nm}$, emission $\sim 520 \mathrm{~nm}$ ).

5) Subtract the fluorescence value of the reagent blank from that of each of the samples. Use corrected data to generate a standard curve of fluorescence versus DNA concentration.

6) Dilute the experimental DNA solution in TE to a final volume of $1.0 \mathrm{ml}$ in disposable cuvettes or test tubes.

7) Add $1.0 \mathrm{ml}$ of the aqueous working solution of the Quant-iT ${ }^{\mathrm{TM}}$ PicoGreen $^{\circledR}$ Reagent to each sample. Incubate for 2 to 5 minutes at RT, protected from light.

8) Measure the fluorescence of the sample using instrument parameters that correspond to those used when generating your standard curve. To minimize photobleaching effects, keep the time for fluorescence measurement constant for all samples.

9) Subtract the fluorescence value of the reagent blank from that of each of the samples. Determine the DNA concentration of the sample from the standard curve generated previously.

10) The assay may be repeated using a different dilution for the sample to confirm the quantitation results. 